\title{
Calibration of the Minos Detectors and Extraction OF NEUTRINO Oscillation PARAMETERS
}

\author{
Christopher B. Smith \\ University College London
}

Submitted to the University of London

in fulfilment of the requirements

for the award of the degree of

Doctor of Philosophy.

October 2002. 
ProQuest Number: U642708

All rights reserved

INFORMATION TO ALL USERS

The quality of this reproduction is dependent upon the quality of the copy submitted.

In the unlikely event that the author did not send a complete manuscript and there are missing pages, these will be noted. Also, if material had to be removed, a note will indicate the deletion.

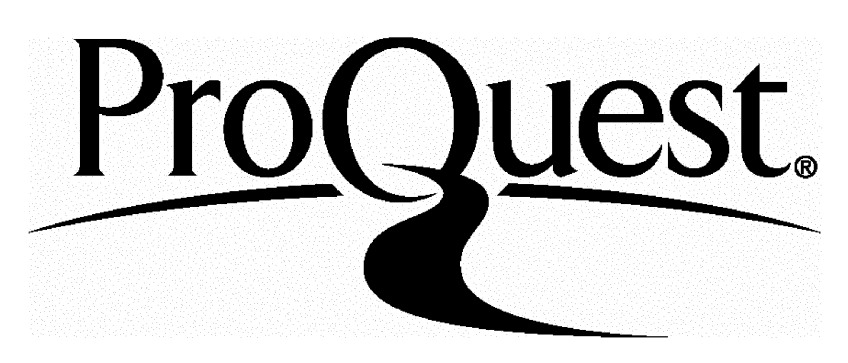

ProQuest U642708

Published by ProQuest LLC(2015). Copyright of the Dissertation is held by the Author.

All rights reserved.

This work is protected against unauthorized copying under Title 17, United States Code. Microform Edition (c) ProQuest LLC.

\author{
ProQuest LLC \\ 789 East Eisenhower Parkway \\ P.O. Box 1346 \\ Ann Arbor, MI 48106-1346
}




\section{Abstract}

The MINOS experiment is designed to search for neutrino oscillations. A neutrino beam created at Fermilab will be sampled first by the Near Detector, on-site at Fermilab, and then by the Far Detector, $735 \mathrm{~km}$ away in the Soudan mine, Minnesota. By comparing the relative fractions of neutrino flavours at the two locations, oscillation parameters can be measured.

Relative calibration between the Near and Far Detectors is achieved using a Light Injection system and cosmic ray muons. Absolute calibration is established using a third, smaller detector: the Calibration Detector, (CalDet), scheduled to run in a series of test-beams at CERN. The CalDet will characterize the response of hadrons, electrons and muons in the MINOS detectors.

Relative calibration of the CalDet using the MINOS Light Injection system and cosmic ray muons has been demonstrated to $2 \%$. Stability measurements of the detector response were made using cosmic ray muons and light output variations of $\sim 2 \%$ over a two week period have been observed which correlate well with local temperature changes.

Simulated charged current $\nu_{\mu}$ and $\bar{\nu}_{\mu}$ interactions in the Near and Far Detectors for the Low Energy NuMI beam have been used to study oscillation parameter measurement with MINOS. Using only the muon energy from quasi-elastic interactions, the parameters have been measured to $7.6 \%$ for $\sin ^{2} 2 \theta$ and $9.6 \%$ for $\Delta m^{2}$ after a two year exposure with MINOS. 


\section{Acknowledgements}

I would like to thank my supervisor, Jenny Thomas, for guidance, advice and support throughout my PhD. I am grateful to Ryan Nichol and Trisha Vahle who provided assistance with some of the plots in Chapter 6. Also, I would like to thank David Petyt for advice and discussion on the work presented in Chapter 7.

At the CalDet, I have had the benefit of working with a great team of people: Phil Adamson, Leo Jenner, Karol Lang, Mike Kordosky, Rob Morse, Ryan Nichol, Ruben Saakyan, Jenny Thomas and Trisha Vahle, among others. Without their collaboration the work in this thesis would not have been possible.

I have also had the pleasure of spending many hours in front of a computer, in an office at UCL with Jon Couchman, Ben West, Ryan Nichol and Leo Jenner, all of whom have helped make my $\mathrm{PhD}$ an enjoyable experience.

Finally, I would like to thank my family, in particular my mother, Dawn and my brother, Mark for continued support and encouragement throughout my student days. 


\section{Contents}

1 Introduction 16

2 Neutrinos in the Standard Model and Beyond 20

2.1 The Standard Model of Particle Physics . . . . . . . . . . 20

2.1 .1 The Fermions .................. 21

2.1 .2 The Bosons ................... 23

2.1.3 Mass in the Standard Model . . . . . . . . . 26

2.2 Massive Neutrinos . . . . . . . . . . . . . . . . 31

2.2.1 Dirac and Majorana Mass . . . . . . . . . . 31

2.2.2 Adding Neutrino Mass to the Standard Model . . . . 32

2.2.3 Neutrino Oscillations in Vacuum . . . . . . . . . 33

2.2.4 Matter-Enhanced Oscillations - The MSW Effect . . . 36

3 Experimental Searches for Neutrino Oscillations 43

3.1 Solar Neutrino Experiments . . . . . . . . . . . . . 43

3.1 .1 SNO ........................ 47

3.2 Atmospheric Neutrino Experiments . . . . . . . . . . . 48

3.2 .1 Super-Kamiokande . . . . . . . . . . . 50

3.3 Reactor Neutrino Experiments . . . . . . . . . . . . . . 52

3.4 Accelerator Neutrino Experiments . . . . . . . . . . . 52 
3.4.1 Short Baseline Experiments .......... . 53

3.4.2 Long Baseline Experiments . . . . . . . . . 54

3.5 Summary ........................ 56

4 The NuMI-MINOS Experiment 59

4.1 The Beam . . . . . . . . . . . . . . . . 59

4.2 The Detectors . . . . . . . . . . . . . . . . 62

4.2.1 MINOS Detector Technologies . . . . . . . . 62

4.2.2 The Far Detector . . . . . . . . . . . . . 64

4.2.3 The Near Detector . . . . . . . . . . . 66

4.3 Physics Capabilities . . . . . . . . . . . . . . . 69

5 Calibration Overview $\quad 74$

5.1 Importance of Calibration . . . . . . . . . . . . 74

5.2 Relative Calibration ............... 75

5.2 .1 Charge Injection ............... 75

5.2 .2 Light Injection . . . . . . . . . . . 76

5.2 .3 Strip-to-Strip Calibration . . . . . . . . 78

5.2 .4 Stopping Muons . . . . . . . . . . . . 79

5.3 Absolute Calibration . . . . . . . . . . . . . 79

6 The Calibration Detector $\quad 81$

6.1 Description of the CalDet . . . . . . . . . . 81

6.1.1 The Test Beam ................ 85

6.2 Calibration Tools . . . . . . . . . . . . 85

6.2 .1 Event Display . . . . . . . . . . . 86

6.2 .2 Low Light Level LI Spectra . . . . . . . . . . . 86

6.2 .3 Track Finder . . . . . . . . . . . . 88

6.2.4 Track Finder Performance . . . . . . . . . . . 91 
6.2.5 A Simple Description of Muon Energy Spectra . . . . 99

6.2.6 Strip End Characterization . . . . . . . . . . 100

6.3 Calibration Procedures . . . . . . . . . . . . . 105

6.3 .1 Event Selection ................. 105

6.3.2 LI Drift Point Corrections . . . . . . . . . . . 108

6.3.3 Angle Corrections . . . . . . . . . . . . . . . . 112

6.3.4 Attenuation Corrections . . . . . . . . . . 115

6.4 Results . . . . . . . . . . . . . . . . 118

6.4.1 Calibration Constants .............. 118

6.4.2 Uniformity of Detector Response . . . . . . . . 118

6.4.3 Stability of Cosmic MEU over Short Time Scales . . . 119

6.4.4 Application of Strip-to-Strip Calibration . . . . . . 123

6.4.5 Beam Muon Characteristics . . . . . . . . . . 128

6.5 Summary . . . . . . . . . . . . . . . 131

7 Extraction of Neutrino Oscillation Parameters 132

7.1 Beam Neutrino Flux . . . . . . . . . . . . . . 133

7.2 GEANT simulation . . . . . . . . . . . . 137

7.3 Muon Reconstruction . . . . . . . . . . . . . . . 141

7.4 Oscillation Parameter Measurement . . . . . . . . . . . . 142

7.5 Other Measurements using Muons . . . . . . . . . . . . . 149

7.6 Beam Misidentification . . . . . . . . . . 156

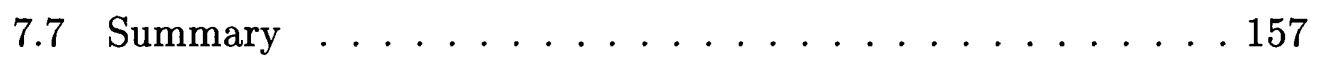

8 Conclusions 159

A A Function to describe the Observed Energy Loss by Muons in Scintillator $\quad 162$

A.1 Muon Energy Spectra . . . . . . . . . . . . . . 162 
A.2 Simulations . . . . . . . . . . . . . . 164

A.3 The Function . . . . . . . . . . . . . . . . . . . 164

A.4 Performing Fits with the Function . . . . . . . . . . . 169

A.5 Summary ......................... 171 


\section{List of Figures}

1.1 The MINOS experiment. . . . . . . . . . . . 18

2.1 The Higgs potential . . . . . . . . . . . . . . . . 29

2.2 Oscillation Probability as a function of neutrino energy for the MINOS baseine. . . . . . . . . . . . . . 37

2.3 Feynman diagram for neutral current neutrino interactions with nucleons (quarks) and electrons in matter. . . . . . . . 38

2.4 Feynmann diagrams for charged current electron neutrinos and anti-neutrinos with electrons in matter. . . . . . . . 39

2.5 Resonant enhancement of neutrino oscillations due to the MSW effect. . . . . . . . . . . . . . . . 41

$2.6 \nu_{\mu} \rightarrow \nu_{e}$ MSW oscillation probability for the MINOS baseline for different values of $\theta$, the mixing angle in vacuum. . . . . . 42

3.1 The predicted solar neutrino energy spectrum showing the energy thresholds for a number of solar neutrino experiments. . . 45

3.2 Measured neutrino fluxes from a number of solar neutrino experiments. ...................... 46

3.3 Measurements of the ratio of ratios, $\mathcal{R}$ for a number of solar neutrino experiments. . . . . . . . . . . . . 50 
3.4 A sketch illustrating the variations in distance traveled by atmospheric neutrinos with zenith angle. . . . . . . . . . . 51

3.5 The allowed parameter space for the LSND results. . . . . . . 55

3.6 Allowed and excluded regions of parameter space for $\nu_{\mu} \rightarrow \nu_{\tau}$

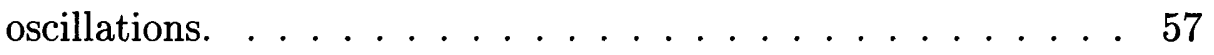

3.7 Allowed and excluded regions of parameter space for $\nu_{\mu} \rightarrow \nu_{e}$ oscillations. . . . . . . . . . . . . . . . 58

4.1 The NuMI beamline. . . . . . . . . . . . . . . . . . . 60

4.2 The expected Far Detector $\nu_{\mu}$ CC energy spectra for the Low, Medium and High energy neutrino beams. . . . . . . . . . . 61

4.3 A length of MINOS scintillator illuminated by a blue LED. . . 63

4.4 A photograph of the Far Detector during construction. . . . . 65

4.5 A sketch of a Near Detector plane. . . . . . . . . . . . . 67

4.6 The instrumented regions of the Near Detector. . . . . . . . 68

4.7 Sketches of event topologies in the MINOS detectors. . . . . . 70

4.8 Sensitivity of MINOS to $\nu_{\mu} \rightarrow \nu_{\tau}$ oscillations from the T-test measurement. . . . . . . . . . . . . . . . 71

$4.990 \%$ exclusion limits from MINOS for $\nu_{\mu} \rightarrow \nu_{x}$ oscillations from a measurement of the $\nu_{\mu}$ CC energy spectrum after 2 years running and for the Low energy beam. . . . . . . . . . 73

$4.1090 \%$ exclusion limits from MINOS for $\nu_{\mu} \rightarrow \nu_{e}$ oscillations from a measurement of the $\nu_{e} \mathrm{CC}$ appearance after 2 years running. . . . . . . . . . . . . . . . . . 73

5.1 A schematic diagram of the Light Injection system. . . . . . 77

6.1 A Photograph of the Calibration Detector. . . . . . . . . . . 82 
6.2 An Example of the event display developed for the Calibration Detector. . . . . . . . . . . . . . . . . . . . . 87

6.3 An example of a fit to a constant. low light level spectrum. . . 89

6.4 An example of the searching pattern used to find track related hits in the CalDet. . . . . . . . . . . . . . . . 90 90

6.5 Fraction of muon and pion events selected using the track finder algorithm for a range of energies in a Monte Carlo simulation of the CalDet. . . . . . . . . . . . . . . . 92

6.6 Last hit plane for $2 \mathrm{GeV}$ muon and pion events selected by the track finder algorithm in a Monte Carlo simulation of the CalDet. . . . . . . . . . . . . . . . . . . . 93

6.7 Stopping plane for tracked CalDet events in a $2 \mathrm{GeV}$ run in the $\mathrm{T} 11$ test-beam. . . . . . . . . . . . . . . . 95

6.8 The fraction of planes hit for through-going muon events in the CalDet. . . . . . . . . . . . . . . . . . . . . 96

6.9 Tracking inefficiencies as a function of plane for through-going muons. . . . . . . . . . . . . . . . . . . . . 9 97

6.10 Fraction of double-ended hits compared to total number of tracked hits for through-going muons. . . . . . . . . . . . 98

6.11 An example of the approximate convolution of a Landau with Poisson. . . . . . . . . . . . . . . . . . . . . . . . 100

6.12 A fit to a muon energy spectrum. . . . . . . . . . . . . . 101

6.13 Examples of techniques that can be used for strip-end characterization. . . . . . . . . . . . . . . . . . . . . . . . 102

6.14 Comparison of the strip-end characterization techniques. . . . 103

6.15 Comparison of the strip-end characterization techniques with varying numbers of entries in the strip-end energy spectra. . . 104 
6.16 Angular distributions of muons in the three usual running conditions of the CalDet. . . . . . . . . . . . . . . . 107

6.17 The average variation in gain for all phototube pixels over the period of $\sim 2$ days. . . . . . . . . . . . . . . 109

6.18 Gain curves showing the linearity of the phototube response with light level for a particular channel. . . . . . . . . . . . . 110

6.19 Demonstration of the ability of the LI system to correct for gain drift to better than $1 \% \ldots \ldots \ldots \ldots$

6.20 A schematic diagram illustrating the dependence of pathlength through a scintillator strip on the angle of the track. . . 113

6.21 Normalized mean ADC as a function of path-length in the scintillator. . . . . . . . . . . . . . . . . . 115

6.22 Variation in path-length corrected response. . . . . . . . . 116

6.23 The dependence of muon pulse height with position along a scintillator strip with and without corrections. . . . . . . . 117

6.24 Variations in the phototube gain at the CalDet. . . . . . . . . 119

6.25 The light output of the CalDet readout channels in terms of photoelectrons. . . . . . . . . . . . . . . . . . 120

6.26 Variations in calibration constants over a two week period. . . 121

6.27 Observed shifts in calibration constants plotted with those expected due to local temperature variations. . . . . . . . . . . 122

6.28 The result of strip-end characterization for a muon sample corrected for strip-to-strip variations. . . . . . . . . . . . . . . 123

6.29 The mean muon hit response in ADCs with and without calibration for the green and clear fibre readout channels. . . . . . 125

6.30 The summed ADC distributions for a $2 \mathrm{GeV}$ electron sample without calibration. . . . . . . . . . . . . . . . . . 126 
6.31 The summed MEU distributions for a $2 \mathrm{GeV}$ electron sample after strip-to-strip calibration. . . . . . . . . . . . . 127

6.32 Range versus beam momentum for stopping muons in the CalDet. . . . . . . . . . . . . . . . . . . . . 129

6.33 Muon $\mathrm{dE} / \mathrm{dx}$ versus plane for $2 \mathrm{GeV}$ beam muons at CalDet. . 130

7.1 The predicted Near and Far $\nu_{\mu}$ CC event rates calculated using four different hadron production models, (no oscillations). . . 135

7.2 The predicted Far/Near ratio for the normalized $\nu_{\mu} \mathrm{CC}$ event rates calculated using four different hadron production models, (no oscillations). . . . . . . . . . . . . . . . 136

7.3 The predicted Near and Far muon energy spectra from $\nu_{\mu}, \bar{\nu}_{\mu}$ CC interactions calculated using four different hadron production models, (no oscillations). . . . . . . . . . . . . . . 139

7.4 The predicted muon energy spectrum from $\nu_{\mu}, \bar{\nu}_{\mu}$ CC interactions with and without oscillations using the GFLUKA hadron production model in the Far Detector. . . . . . . . . . . 140

7.5 The normalized muon event rate ratios for the GFLUKA hadron production model. . . . . . . . . . . . . . . . . 140

7.6 A $\chi^{2}$ fit to the data using the MC sample weighted with many different values for the oscillation parameters, (event rates predicted by the GFLUKA model). . . . . . . . . . . . . . . . . 144

7.7 The normalized muon energy spectra for the data and the MC best fit. . . . . . . . . . . . . . . . . . . . . 145

7.8 The confidence limits after 2 years data taking on the neutrino oscillation parameters measured using the reconstructed muon energy spectrum, (GFLUKA hadron production model). . . . 146 
7.9 The $90 \%$ confidence limits after 2 years data taking on the neutrino oscillation parameters measured using the reconstructed muon energy spectrum for four hadron production models: GFLUKA, BMPT, MARS and MALENSEK. . . . . . . . 147

7.10 Differences in the predicted $\nu_{\mu} \mathrm{CC}$ energy spectrum at the Far Detector between different hadron production models (no oscillations) . . . . . . . . . . . . . . . 148

7.11 The fraction of neutrino events accepted for a range of maximum shower energies. . . . . . . . . . . . . . 150

7.12 The $90 \%$ confidence limits after 2 years data taking on the neutrino oscillation parameters measured using the reconstructed muon energy spectrum for a range of cuts on the shower energy in the event. . . . . . . . . . . . . . . . 151

7.13 Muon energy spectra in the Far Detector without oscillations for a range of cuts on the maximum shower energy in an event. 152

7.14 The $90 \%$ confidence limits after 2 years data taking on the oscillation parameters measured using the muon and neutrino energy spectra. . . . . . . . . . . . . . 153

7.15 The $90 \%$ confidence limits after 2 years data taking on the oscillation parameters measured using the muon energy spectrum from quasi-elastic CC events showing the effect of systematic errors in the $y$-distribution. . . . . . . . . . . 154

$7.1690 \%$ confidence limits showing the effect of misidentifying the hadron production model. . . . . . . . . . . . 157

A.1 A muon energy spectrum measured at the Calibration Detector.163

A.2 GEANT simulation of the energy loss in scintillator for a 3.5 $\mathrm{GeV}$ muon. . . . . . . . . . . . . . . . . 165 
A.3 A simulated muon energy spectrum showing features similar to those observed at the Calibration Detector. . . . . . . 166

A.4 A simulated muon energy spectrum showing the contributions from each of the photoelectron peaks. . . . . . . . . . 167

A.5 A fit to the first photoelectron peak using a continuous Poisson distribution including a factor to scale the x-axis. . . . . . 168

A.6 A fit to a simulated muon energy spectrum. . . . . . . . . 170

A.7 A fit to a CalDet muon energy spectrum . . . . . . . . . 171 


\section{List of Tables}

2.1 Some properties and quantum numbers of the leptons. . . . . 22

2.2 Some properties and quantum numbers of the quarks. . . . . . 24

6.1 Selection statistics from a CalDet T11 Beam run at $2 \mathrm{GeV}$. . 94

7.1 Percentage errors on oscillation parameter measurements for different hadron production models for 2 years of data. . . . 155 


\section{Chapter 1}

\section{Introduction}

The Standard Model of Particle Physics has been extremely successful in describing and predicting the interactions and properties of fundamental particles. However, in recent years, it has been recognized that experimental results cannot be fully understood within the context of the current theory. One such field is that of neutrino physics.

The neutrino is a spin- $1 / 2$ fermion; it is colourless, carries zero electromagnetic charge and weak isospin of $+\frac{1}{2}$. The neutrino can therefore only interact weakly, justifying its reputation for being elusive. It is a lepton and accordingly, in the Standard Model, exists in three flavours: electron, muon and tau. The most controversial issue regarding the neutrino is the value of its mass. The Standard Model states that the neutrino masses are all exactly zero, a direct consequence of this being that right handed chiral neutrinos have no allowed gauge interactions since the weak interaction maximally violates parity.

However, there is evidence for a disagreement between the measured numbers of neutrinos arriving at the Earths surface compared to the predicted numbers based on Standard Model physics. Many experiments, including 
Super-Kamiokande [1][2], a $50 \mathrm{kT}$ water-Cerenkov detector and SNO [3][4] a heavy water Cerenkov detector, have investigated two isolated theoreticalexperimental discrepancies:

- The Solar neutrino problem highlights the discrepancy between the measured number of $\nu_{e}$ arriving from the sun compared to the number predicted by the Solar Model. Both SNO and Super-Kamiokande, among others, have reported results regarding this.

- The Atmospheric neutrino problem addresses the ratio of the number of $\nu_{\mu}$ to $\nu_{e}$ reaching the Earth's surface from the upper atmosphere as a result of cosmic ray collisions with nuclei. The theoretical ratio for this is approximately 2:1 in favour of $\nu_{\mu}$. Experiments fail to measure this value and, in particular, Super-Kamiokande has reported that the discrepancy in the ratio is due to missing $\nu_{\mu}$.

One possible solution to these discrepancies can be formulated from another area of particle physics. The phenomenon of flavour mixing for hadrons is observed in experiments and described by the Standard Model. The weak interaction eigenstates for hadrons are not the same as the mass eigenstates. Following a weak interaction, the hadron is in a state which is a superposition of a number of mass eigenstates and an observation of the hadron shows it to be in a state ultimately given by the Cabbibo-Kobayashi-Maskawa (CKM) mixing matrix. The implications of this to neutrino physics is that, from a theoretical point of view, a similar freedom for leptons may be expected.

In 1967, Pontecorvo [5][6][7][8] first considered the consequences of this potential freedom to neutrinos. He postulated that if neutrinos had a finite mass, and also that the mass and weak eigenstates were not identical, then a mixing matrix could be formulated. In this scenario, any neutrino pro- 
duced in an interaction is in a state which is a coherent superposition of all three mass eigenstates. Since neutrinos have no equivalent to hadronization in quarks, they continue to propagate through space as a superposition of states until they interact again. Manifestations of this effect can be observed by current experiments such as Super-Kamiokande and SNO and limits on the mixing matrix parameters can be set. However, precise measurements are extremely difficult as experiments dealing with atmospheric and solar neutrinos have no control over the neutrino sources.

An alternative approach to solving the neutrino problem is taken by longbaseline experiments, such as the Main Injector Neutrino Oscillation Search (MINOS) experiment. A beam of neutrinos created at Fermi National Accelerator Laboratory (FNAL) will be fired through the Earth towards Minnesota. On their way, a small fraction of these particles interact in the MINOS calorimeters. The Near Detector, on site at FNAL, samples the beam before oscillations occur. The Far Detector, located in the Soudan mine $735 \mathrm{~km}$ away in Northern Minnesota, then makes another measurement of the beam, allowing a comparison of the neutrino events at the two locations to be made. From such measurements, oscillation parameters can be estimated. Figure 1.1 shows a schematic diagram of the experiment.

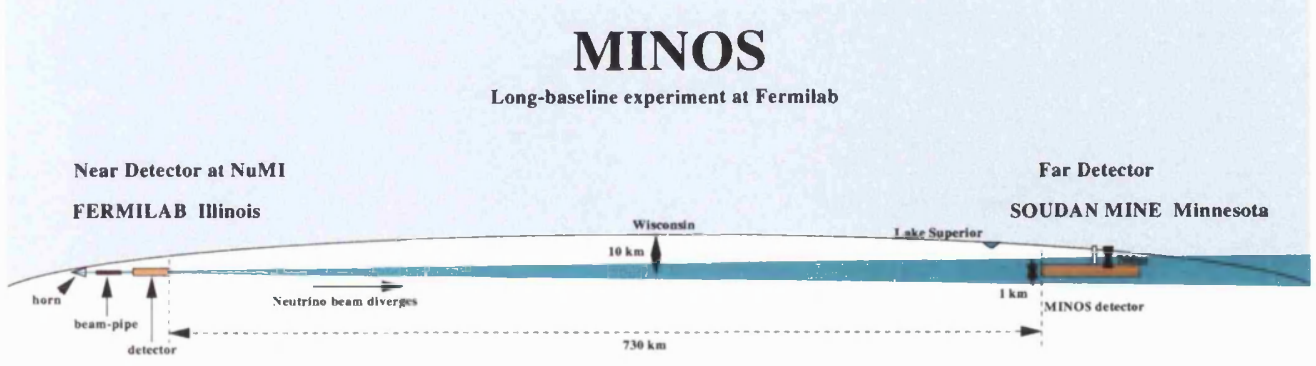

Figure 1.1: The MINOS experiment. 
When comparing responses from two detectors as MINOS does, relative calibration is very important; and absolute calibration is essential for accurately measuring the oscillation parameters. MINOS is designed to achieve a $2 \%$ relative calibration and a $5 \%$ absolute calibration. To attain this, a light injection system and cosmic ray muons are used to monitor and account for detector-detector differences. To achieve absolute calibration, a third MINOS detector has been built: the Calibration Detector, (CalDet). The same in basic design as the Near and Far detectors but smaller, the CalDet is being used to characterize the response of hadrons, electrons and muons of known energies in the MINOS detectors. The CalDet is situated in the East hall at CERN where it is to be exposed to several beams of different energies over three years of running (2001-2003).

Chapters 6 and 7 constitute the work that is being presented for this degree although within these chapters some of the work was carried out in conjunction other collaborators. In Chapter 6, the Light Injection fits in Section 6.2.2, the generation of Monte Carlo beam muons in Section 6.2.4, the Light Injection corrections in Section 6.3.2 and the selection of an electron sample in Section 6.4.4 were accomplished with the assistance of others. In Chapter 7, the predicted neutrino fluxes in Section 7.1 and the Monte Carlo events described in Section 7.2 were obtained from the collaboration for use in the analysis. 


\section{Chapter 2}

\section{Neutrinos in the Standard}

\section{Model and Beyond}

The Standard Model has been extremely successful in predicting many observable quantities in Particle Physics. Rigorous testing by experiments at all achievable energies has left the theory intact and the basic properties of nature that it defines are necessarily incorporated into any new theories. New theories however are sought, as the Standard Model does not attempt to describe gravity, and many of its parameters are arbitrarily set, in particular the particle masses, rather than falling naturally from fundamental principles. Nevertheless, it is essential to explain the ideas central to the Model before describing possible extensions to it.

\subsection{The Standard Model of Particle Physics}

The Standard Model was formulated to describe the observed interactions of known particles. The Model assumes that all experimental signatures are the result of a small number of fundamental particles interacting via three 
fundamental forces: the strong force, weak force and electromagnetic force. The fundamental particles are divided into two distinct groups: fermions and gauge bosons. They differ from each other in terms of their intrinsic angular momentum or spin: the fermions are 1/2-integer spin particles and are the constituents of matter; the bosons are integer spin particles and are responsible for carrying and transmitting force.

\subsubsection{The Fermions}

The fermions can be further subdivided into leptons and quarks. The quarks feel the strong force whereas the leptons do not. For both groups, 6 particles and their anti-particles exist which can be further subdivided into 3 generations. Within the generation, the 2 fermions are separated by 1 charge unit.

\section{Leptons}

There are two types of lepton, distinguishable by their electromagnetic charge. The two most familiar leptons are the negatively charged electron and its partner, the electromagnetically neutral electron neutrino. These particles form the first lepton generation. The second and third generations contain similar particles, differentiable by the mass of the charged lepton, which increases with each generation. The second generation is comprised of the muon and muon neutrino and the third by the tau and the tau neutrino. Table 2.1 outlines the lepton generations and some properties of the particles.

The electron is a stable particle whereas the muon and tau are not. The heavier leptons can decay to produce the lighter flavours. The tau is also massive enough to decay to quarks. The limits on the neutrino masses in Table 2.1 are obtained from measurements of energy spectra end-points. For 
$\nu_{e}$, beta decays from atomic tritium are observed; for $\nu_{\mu}$, muons from pion decay at rest are similarly used; and for $\nu_{\tau}$, analysing certain decays of $\tau$ to pions and a $\nu_{\tau}$ yields an upper limit on the mass.

\begin{tabular}{|c|c|c|c|c|l||}
\hline Gen. & Flavour & $\mathrm{Q}$ & $\left(\mathrm{T}_{3}\right)_{L}$ & $\begin{array}{c}\text { Mass } \\
(\mathrm{MeV})\end{array}$ & Lifetime \\
\hline \hline \multirow{2}{*}{1} & $\mathrm{e}$ & -1 & $-\frac{1}{2}$ & 0.51 & $>4.2 \times 10^{24} \mathrm{yr}, \mathrm{CL}=68 \%$ \\
\cline { 2 - 6 } & $\nu_{e}$ & 0 & $+\frac{1}{2}$ & $<3 \times 10^{-6}$ & $\begin{array}{l}>21 \times 10^{9} \mathrm{~s} \text { (solar) } \\
>900 \mathrm{~s}, \mathrm{CL}=90 \% \text { (reactor) }\end{array}$ \\
\hline \multirow{2}{*}{2} & $\mu$ & -1 & $-\frac{1}{2}$ & 105.7 & $2.2 \times 10^{-6} \mathrm{~s}$ \\
\cline { 2 - 7 } 3 & $\nu_{\mu}$ & 0 & $+\frac{1}{2}$ & $<0.19$ & $>2.9 \mathrm{~s}, \mathrm{CL}=90 \%$ \\
\cline { 2 - 7 } & $\tau$ & -1 & $-\frac{1}{2}$ & 1777 & $291 \times 10^{-15} \mathrm{~s}$ \\
\hline
\end{tabular}

Table 2.1: Some properties and quantum numbers of the leptons. $Q$ is the electromagnetic charge of the particle in units of $e$, the charge on the positron; $T_{3}$ is the weak isospin of the particle, the quantum number for the weak force. The subscript $\mathrm{L}$ indicates that only left handed chiral particles and right handed anti-particles feel the weak force. The quantum numbers for the anti-particles are obtained by multiplying those shown by -1 . For full listings consult the Review of Particle Physics [9].

\section{Quarks}

Quarks are the only fermions which feel all 3 forces. They carry colour, the charge of the strong force, they are charged weakly and electromagnetically and are massive. They are grouped into 3 generations, with the hierarchy following the mass of the particles. Quarks carry only fractional electromagnetic charge; either $+2 / 3$ or $-1 / 3$, and each generation has one particle of 
each charge. The quarks are named up $(\mathrm{u}), \operatorname{down}(\mathrm{d}), \operatorname{charm}(\mathrm{c}), \operatorname{strange}(\mathrm{s})$, top(t) and bottom(b) and are arranged generationally as follows:

$$
\left(\begin{array}{l}
u \\
d
\end{array}\right)\left(\begin{array}{l}
c \\
s
\end{array}\right)\left(\begin{array}{l}
t \\
b
\end{array}\right)
$$

Quarks interact strongly according to the colour charge that they carry. A quark can carry one of three colours: $\operatorname{red}(\mathrm{r})$, green(g) or blue(b); $(\bar{r}, \bar{g}, \bar{b}$ for anti-quarks). A peculiar property of the strong force, explained in the next section, means that quarks are never observed in isolation. Following the production of a quark in an interaction, further quarks are captured from the vacuum such that the resulting bound collection of particles (called a hadron) is colourless and carries an integer electromagnetic charge. There are two types of hadron: a baryon consists of three quarks, one of each colour, a common example being the proton (uud). A meson is made up from one particle and one anti-particle each being of the same colour type, for example the $\pi^{+}(\mathrm{u} \bar{d})$.

Table 2.2 shows some of the basic quark properties and quantum numbers.

\subsubsection{The Bosons}

The interactions of the fundamental particles are described in terms of the exchange of a gauge boson. Each of the forces is represented by one or more of these bosons, the properties of which influence the macroscopic manifestations of the forces.

The electromagnetic force couples proportionally to the charge of the particles, thus only charged particles feel the effect of this force. It is mediated by the massless, chargeless photon $(\gamma)$, and consequently the electromagnetic force has an infinite range. 


\begin{tabular}{|c|c|c|c|c|l||}
\hline Gen. & Flavour & $\begin{array}{r}\mathrm{Q} \\
(\mathrm{e})\end{array}$ & $\left(\mathrm{T}_{3}\right)_{L}$ & Colour & $\begin{array}{l}\text { Mass } \\
(\mathrm{MeV})\end{array}$ \\
\hline \hline \multirow{2}{*}{1} & $\mathrm{u}$ & $+\frac{2}{3}$ & $+\frac{1}{2}$ & $\mathrm{r}, \mathrm{g}, \mathrm{b}$ & $1.5-5$ \\
\cline { 2 - 6 } & $\mathrm{d}$ & $-\frac{1}{3}$ & $-\frac{1}{2}$ & $\mathrm{r}, \mathrm{g}, \mathrm{b}$ & $17-27$ \\
\hline \multirow{2}{*}{2} & $\mathrm{c}$ & $+\frac{2}{3}$ & $+\frac{1}{2}$ & $\mathrm{r}, \mathrm{g}, \mathrm{b}$ & $1100-1400$ \\
\cline { 2 - 6 } & $\mathrm{s}$ & $-\frac{1}{3}$ & $-\frac{1}{2}$ & $\mathrm{r}, \mathrm{g}, \mathrm{b}$ & $60-170$ \\
\hline \multirow{2}{*}{3} & $\mathrm{t}$ & $+\frac{2}{3}$ & $+\frac{1}{2}$ & $\mathrm{r}, \mathrm{g}, \mathrm{b}$ & 173800 \\
\cline { 2 - 6 } & $\mathrm{b}$ & $-\frac{1}{3}$ & $-\frac{1}{2}$ & $\mathrm{r}, \mathrm{g}, \mathrm{b}$ & $4100-4400$ \\
\hline
\end{tabular}

Table 2.2: Some properties and quantum numbers of the quarks. $Q$ is the electromagnetic charge of the particle in units of $e$, the charge on the positron; $T_{3}$ is the weak isospin of the particle; Colour is the quantum number for the strong force.

The strong force is mediated by eight gluons and couples to the colour charge possessed by quarks. Gluons are massless and therefore one may expect the strong force to have an infinite range. However, a further property of the gluons is that they also possess colour charge. A consequence of this is that the strength of the strong force is constant with distance. It is for this reason that quarks are never seen in isolation. As the separation of a quark from its hadron increases, the potential energy in the bond becomes large enough to create quark-antiquark pairs, the result being that no coloured quark composite can ever exist.

The weak force is mediated by three massive bosons: $\mathrm{W}^{+}, \mathrm{W}^{-}$and $\mathrm{Z}^{\circ}$. The $\mathrm{W}^{ \pm}$have a mass of $80.45 \mathrm{GeV}$ and the $\mathrm{Z}^{\circ}$ has a mass of $91.2 \mathrm{GeV}$. For this reason, weak interactions are suppressed at low energies. This is reflected in decays such as $n \rightarrow p+e^{-}+\bar{\nu}$ in which the neutron has a lifetime of $\sim 15$ minutes which is many orders of magnitude longer than particles decaying via the electromagnetic or strong forces. It is this distinction that 
earned it the name weak, even though later, the weak and electromagnetic forces were found to be manifestations of the same electroweak force.

The weak force is unique in that it is felt by all fermions, coupling to the weak isospin, and, due to the electromagnetic charge on the $\mathrm{W}$ bosons, gives flavour changing transitions. The $\mathrm{Z}$ boson couples to both weak isospin and electromagnetic charge.

Weak interactions are known to violate parity. Only left-handed fermions and right-handed anti-fermions may take part in weak interactions. Indirect observations of neutrinos from $\beta$-transitions of polarized cobalt nuclei:

$$
{ }^{60} \mathrm{Co} \rightarrow{ }^{60} N i^{*}+e^{-}+\bar{\nu}_{e}
$$

showed that the neutrinos were only ever produced in a certain direction with respect to the direction of polarization of the cobalt [10]. In the SM, this maximal parity violation is interpreted as neutrinos only being produced with a certain helicity, where helicity is defined as the projection of the momentum unit vector onto the spin vector of the particle. If the neutrino is massless, the observed parity violation can be explained. This leads from the fact that neutrinos produced in the left handed chiral state would, for a massless particle, always be observed in the left handed helicity state, since the states are identical in this limit. A massive particle however could sometimes be measured in a right-handed helicity state since it can be boosted into a frame in which its momentum direction and therefore helicity is reversed.

These results, along with the experiments mentioned in Section 2.1.1 which have never measured a non-zero neutrino mass, led to the neutrinos' masses in the Standard Model to be set to zero. 


\subsubsection{Mass in the Standard Model}

The Standard Model is an $\mathrm{SU}(3) \otimes \mathrm{SU}(2)_{L} \otimes \mathrm{U}(1)$ gauge theory. A recurring theme in physics is that a symmetry or invariance in nature leads to a conservation law. This principle is also applied in the Standard Model.

\section{Gauge Symmetries and the Glashow-Weinberg-Salaam Model}

In Quantum Field Theory, particles are defined in terms of their Lagrangians and described by field equations. A local gauge symmetry imposed on a fermion field can yield a term corresponding to its interaction with a boson field. A conserved quantity can then be defined corresponding to the charge associated with the force involved. As an example, consider the Lagrangian for a free fermion:

$$
\mathcal{L}=\bar{\psi}\left(i \gamma^{\mu} \delta_{\mu}-m\right) \psi
$$

where $\psi$ is the field describing the dynamics of the particle and $\mathrm{m}$ is its mass. Demanding a local U(1) gauge symmetry of the form:

$$
\psi \rightarrow \psi^{\prime}=e^{i \alpha(x) Q} \psi
$$

leads to the lagrangian for Quantum Electrodynamics (QED):

$$
\mathcal{L}=\bar{\psi}\left(i \gamma^{\mu} \delta_{\mu}-m\right) \psi-e \bar{\psi} \gamma^{\mu} Q \psi A^{\mu}-\frac{1}{4} F_{\mu \nu} F^{\mu \nu}
$$

where $A_{\mu}$ is the photon field required to preserve the invariance and $F_{\mu \nu}=\delta^{\mu} A^{\nu}-\delta^{\nu} A^{\mu}$. Insisting on local gauge invariance, an interaction term of the fermion field, $\psi$ with the photon field, $A_{\mu}$ of the form:

$$
-e \bar{\psi} \gamma^{\mu} Q \psi A^{\mu}
$$

is unavoidable. $\mathrm{Q}$ is the charge operator whose eigenvalues are conserved quantities; in this case, electromagnetic charge. 
The $\mathrm{SU}(3)$ gauge invariance in the Standard Model is imposed to describe Quantum Chromodynamics (QCD), the theory of strong interactions and colour is the conserved quantity.

The $\mathrm{SU}(2)_{L} \otimes \mathrm{U}(1)$ symmetry leads to the Electroweak theory which unifies the electromagnetic and weak forces. Requiring this invariance introduces four gauge fields. Three of these fields couple to the weak isospin carried by left-handed fermions and the last couples to weak hypercharge carried by all fermions.

The interaction terms introduced to the Lagrangian are then:

$$
-g \bar{\chi}_{L} \gamma_{\mu} \mathbf{T} . \mathbf{W}^{\mu} \chi_{L}-g^{\prime} \bar{\psi} \gamma_{\mu} \frac{Y}{2} \psi B^{\mu}
$$

where $\mathbf{W}^{\mu}$ and $B^{\mu}$ are the fields that were necessarily introduced to preserve the gauge invariance. The operators $\mathbf{T}$ and $\mathrm{Y}$ are the generators of the $\mathrm{SU}(2)_{L}$ and $\mathrm{U}(1)_{Y}$ groups which modify interaction coupling strengths according to the weak quantum numbers of the fields involved, (analagous to the $\mathrm{Q}$ operator in the QED example above).

Since only left-handed fermions are observed to couple to the $\mathrm{W}^{ \pm}$, the fermion fields are separated into left and right chiral projections. The lefthanded fermions are represented as $\mathrm{SU}(2)$ doublets whereas the right-handed are $\mathrm{SU}(2)$ singlets. For the first generations of the fermions, we have the following:

$$
\begin{array}{ll}
\text { Leptons } & \text { Quarks } \\
\chi_{L}^{l}=\left(\begin{array}{c}
\nu_{e} \\
e^{-}
\end{array}\right)_{L} & \chi_{L}^{q}=\left(\begin{array}{c}
u \\
d
\end{array}\right)_{L} \\
\psi_{R}=e_{R}^{-} & \psi_{R}=u_{R}, d_{R}
\end{array}
$$

Note that for quarks the right-handed chiral state is represented by both 
members of the generation, whereas for leptons, the neutrino has no righthanded state.

The four fields each represent a gauge boson, however these are not the $\mathrm{W}, \mathrm{Z}$ and $\gamma$ bosons described earlier. For gauge invariance to hold and for the theory to be renormalizable, these bosons must be massless. Another process is required to connect the gauge fields to the familiar $\mathrm{W}, \mathrm{Z}$ and $\gamma$ and to generate the masses of the $\mathrm{W}$ and $\mathrm{Z}$ bosons. This process is called the Higgs Mechanism.

\section{Spontaneous Symmetry Breaking and the Higgs Mechanism}

Spontaneous Symmetry Breaking (SSB) underlies the Higgs Mechanism. Consider the introduction of four scalar particle fields, $\phi_{i}$, where the $\mathrm{SU}(2) \otimes \mathrm{U}(1)$ gauge invariant Lagragian is given by:

$$
\mathcal{L}=\left|\left(i \delta_{\mu}-g \mathbf{T} \cdot \mathbf{W}_{\mu}-g^{\prime} \frac{Y}{2} B_{\mu}\right) \phi\right|^{2}
$$

where ||$^{2} \equiv()^{\dagger}(\quad)$.

Now assume that the vacuum is not a singlet of the gauge symmetry, but rather there are an infinite number of states with the same ground-state energy. The process of choosing one of these states is known as SSB and in such a case a field can acquire a non-zero vacuum expectation value. For example, Figure 2.1 shows a potential for which this process can happen. The ring of minimum potential at the bottom of the "wine bottle" are the infinite degenerate non-zero vacuum states. This is the Higgs potential and is expressed mathematically as:

$$
V(\phi)=\frac{1}{2} \mu^{2} \phi^{2}+\frac{1}{4} \lambda \phi^{4}
$$

where $\mu^{2}<0$ and $\lambda>0$. 
Adding this term to the Lagrangian above preserves gauge invariance, but causes the scalar fields to acquire a non-zero vacuum expectation value. It also introduces an extra degree of freedom for each field resulting from the degeneracy of the vacuum state, (often expressed as a Goldstone boson which facilitates transitions between these degenerate vacuum states). The
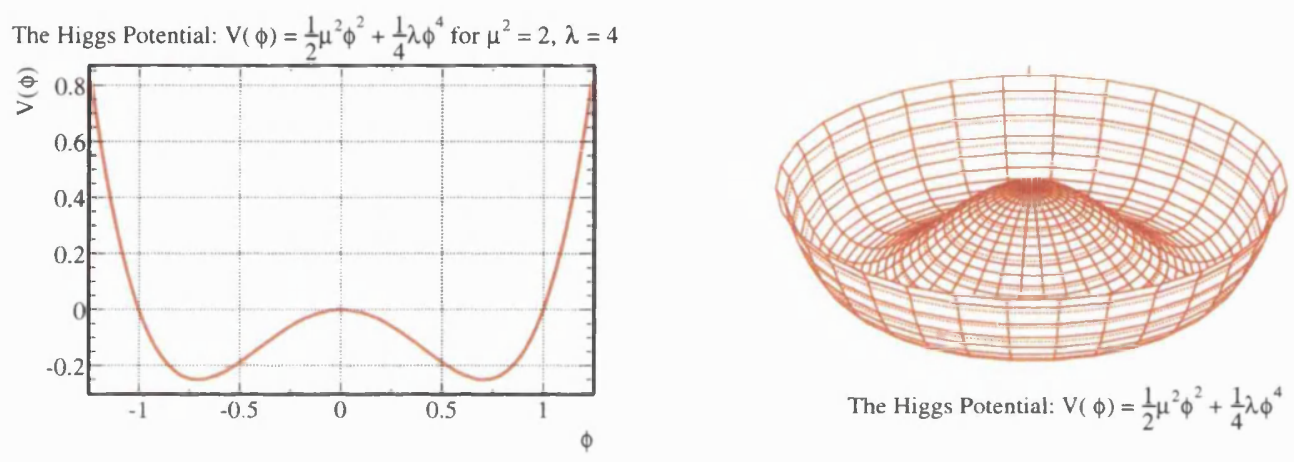

Figure 2.1: The Higgs potential. The left-handed plot shows a 2-D representation of the potential for $\mu^{2}=2$ and $\lambda=4$. The right-handed plot is a 3-D representation showing the "wine bottle" shape of the potential. The ring corresponding to the minimum value of the potential causes the degeneracy of the vacuum state. It is the choice of one of these degenerate states to be the true vacuum that leads to SSB.

scalar fields are expressed as an $\mathrm{SU}(2)$ doublet:

$$
\phi=\left(\begin{array}{c}
\phi^{+} \\
\phi^{0}
\end{array}\right)=\left(\begin{array}{c}
\left(\phi_{1}+i \phi_{2}\right) / \sqrt{2} \\
\left(\phi_{3}+i \phi_{4}\right) / \sqrt{2}
\end{array}\right)
$$

To break the symmetry, we choose a "true" vacuum:

$$
\phi_{0}=\sqrt{\frac{1}{2}}\left(\begin{array}{l}
0 \\
v
\end{array}\right)
$$

where $v=\sqrt{-\mu^{2} / \lambda}$. 
This choice spontaneously breaks the gauge symmetry and the Goldstone bosons are said to be "eaten" by the vector fields to become massive. In fact, the extra degrees of freedom from having degenerate vacua become the longitudinal polarisations for the $\mathrm{W}$ and $\mathrm{B}$ fields.

The $W_{\mu}^{1}$ and $W_{\mu}^{2}$ fields now correspond to the massive $W_{\mu}^{+}$and $W_{\mu}^{-}$gauge fields. The physical $Z_{\mu}$ and $A_{\mu}$ fields however are obtained from mixing the $W_{\mu}^{3}$ and $B_{\mu}$ fields:

$$
\begin{gathered}
A_{\mu}=\cos \theta_{W} B_{\mu}+\sin \theta_{W} W_{\mu}^{3} \\
Z_{\mu}=-\sin \theta_{W} B_{\mu}+\cos \theta_{W} W_{\mu}^{3}
\end{gathered}
$$

where $\theta_{W}$ is the weak mixing angle.

Note that this choice of $A_{\mu}$ has the generators $T^{3}+\frac{Y}{2}=Q$, but $Q \phi_{0}=0$. Therefore although the spontaneous symmetry breaking generates mass for the other three gauge bosons, the vacuum is invariant under the combination $\mathrm{Q}$ and the field $A_{\mu}$ corresponds to the massless photon.

Having introduced the scalar fields to provide a means of generating mass for the $\mathrm{W}$ and $\mathrm{Z}$ bosons, we can now consider its interactions with the fermion fields. Coupling the left-hand doublet and the right hand singlet fermion fields with the Higgs scalar fields produces fermionic mass terms in the Lagranian. For the first lepton generation:

$$
\begin{aligned}
\mathcal{L} & =f^{l} \bar{\chi}_{L}^{l} \phi_{0} e_{R}+f^{l} \bar{e}_{R} \phi_{0} \chi_{R}^{l} \\
& =f^{l} \frac{v}{\sqrt{2}}\left(\bar{e}_{L} e_{R}+\bar{e}_{R} e_{L}\right)
\end{aligned}
$$

where $\mathrm{f}^{l}$ is the Yukawa coupling and $f^{l} \frac{v}{\sqrt{2}}=m_{e}$, the mass of the electron. The neutrino does not have a similar term to the electron as there is no $\nu_{R}$ singlet in the Standard Model. 


\subsection{Massive Neutrinos}

Introducing massive neutrinos is an extension to the Standard Model, however, they can be incorporated with only a minor modification. Neutrino mass can appear in two ways: as a Dirac mass term in the Lagrangian, just as for the charged leptons; or by introducing a Majorana mass term.

\subsubsection{Dirac and Majorana Mass}

The Dirac term assumes that a particle and its anti-particle are different; that is, the right-handed state, $\nu_{R}$ is fundamentally different from $\nu_{R}^{C}$, the CPT partner of $\nu_{L}$. This results in mass terms which connect the $\mathrm{L}$ and $\mathrm{R}$ components of the same field:

$$
\begin{aligned}
\mathcal{L}_{D} & =m_{D} \bar{\nu} \nu \\
& =m_{D}\left(\bar{\nu}_{L} \nu_{R}+\bar{\nu}_{R} \nu_{L}\right)
\end{aligned}
$$

where $\nu \equiv \nu_{L}+\nu_{R}$. Dirac neutrinos conserve lepton number and have four components, $\nu_{L}, \nu_{R}^{C}, \nu_{R}, \nu_{L}^{C}$ analagous to $e_{L}^{-}, e_{R}^{+}, e_{R}^{-}, e_{L}^{+}$for electrons.

Alternatively, a Majorana neutrino can be defined. Since neutrinos are electromagnetically neutral, a neutrino can be its own anti-particle. In other words, the CPT partner of the left-handed neutrino is identical to the righthanded anti-neutrino:

$$
\left(\nu_{L}\right)^{C}=\nu_{R}^{C}
$$

The Majorana mass terms connect the $\mathrm{L}$ and $\mathrm{R}$ components of conjugate fields. The terms appearing in the Lagrangian due to $\nu_{L}$ are then:

$$
\begin{aligned}
\mathcal{L}_{M} & =m_{M} \bar{\chi} \chi \\
& =m_{M}\left(\bar{\nu}_{L} \nu_{R}^{C}+\bar{\nu}_{R}^{C} \nu_{L}\right)
\end{aligned}
$$

where $\chi$ is the self conjugate field: $\chi=\nu_{L}+\nu_{R}^{C}=\chi^{C}$. A similar term can also be expressed for $\nu_{R}$. 


\subsubsection{Adding Neutrino Mass to the Standard Model}

The most general way to incorporate mass into the Standard Model is to introduce a $\mathrm{SU}(2)$ singlet $\nu_{R}$. This right-handed neutrino is "sterile", it has no interactions available to it. Now it is possible to describe a scenario in which there are three different neutrino masses corresponding to the Majorana terms for both $\nu_{L}$ and $\nu_{R}$ as well as the usual Dirac mass.

It is simplest to proceed by expressing $\nu_{L}$ and $\nu_{R}$ in terms of the selfconjugate fields $\chi$ and $\omega$ :

$$
\begin{aligned}
& \chi=\nu_{L}+\left(\nu_{L}\right)^{C} \\
& \omega=\nu_{R}+\left(\nu_{R}\right)^{C}
\end{aligned}
$$

We can write the Lagrangian for neutrino masses as follows:

$$
\mathcal{L}=\bar{n}_{L} \mathcal{M} n_{L}+\text { h.c. }
$$

where

$$
n_{L}=\left(\begin{array}{c}
\chi \\
\omega
\end{array}\right) \text { and } \mathcal{M}=\left(\begin{array}{cc}
m_{L} & \frac{m_{D}}{2} \\
\frac{m_{D}}{2} & m_{R}
\end{array}\right)
$$

with $m_{L}$ and $m_{R}$ being the Majorana masses associated with the left and right handed neutrinos respectively.

Diagonalizing $\mathcal{M}$ to give $\mathcal{M}_{d}=\operatorname{diag}\left(m_{1}, m_{2}\right)$ and introducing the eigenstates $\eta_{1}$ and $\eta_{2}$ such that:

$$
\left(\begin{array}{l}
\chi \\
\omega
\end{array}\right)=\left(\begin{array}{cc}
\cos (\theta) & \sin (\theta) \\
-\sin (\theta) & \cos (\theta)
\end{array}\right)\left(\begin{array}{l}
\eta_{1} \\
\eta_{2}
\end{array}\right)
$$

where the mixing angle, $\theta$ is given by $\tan 2 \theta=\frac{m_{D}}{m_{R}-m_{L}}$. Then the neutrino mass eigenvalues are given by:

$$
m_{1,2}=\frac{1}{2}\left[\left(m_{R}+m_{L}\right) \pm \sqrt{\left(m_{R}-m_{L}\right)^{2}+m_{D}^{2}}\right]
$$




\section{The Seesaw Mechanism}

A special case for this method of including neutrino mass is known as the seesaw mechanism [11][12][13]. It provides an explanation as to why the neutrino mass is so small compared to the other fermions and is therefore theoretically attractive.

Consider the case where $m_{L}=0$ and $m_{D} \ll m_{R}$. The mass eigenstates are then:

$$
\begin{aligned}
& m_{1} \sim \frac{m_{D}^{2}}{m_{R}} \ll m_{D} \\
& m_{2} \sim m_{R}
\end{aligned}
$$

Thus, starting from a typical Dirac mass due to $\nu_{L}$ and a much heavier mass due to $\nu_{R}$ we end up with a very light mass state, $m_{1}$ and a very heavy state, $m_{2}$. It can be seen that it is the heaviness of $\omega$, (made predominantly from $\nu_{R}$ ) which makes $\chi$, (made predominantly from $\nu_{L}$ ) so light.

\subsubsection{Neutrino Oscillations in Vacuum}

Quantum mechanical oscillations are a consequence of flavour mixing for massive fermions. In the Standard Model, because of the degeneracy of the neutrino mass (they are all zero), there is no flavour mixing for the leptons; that is, the flavour and the mass eigenstates are the same. Some possible consequences of non-zero neutrino mass are now considered.

\section{Three Flavour Mixing}

Assume that neutrinos have Dirac mass and that flavour mixing does occur, then the neutrino flavour eigenstates can be written as linear combinations of the mass eigenstates:

$$
\nu_{\alpha}=\sum_{i} U_{\alpha i} \nu_{i}
$$


where $\alpha$ indexes the flavour eigenstates: $\nu_{e}, \nu_{\mu}, \nu_{\tau}$ and $i$ indexes the mass eigenstates $\nu_{1}, \nu_{2}$ and $\nu_{3} . \mathrm{U}$ is a unitary matrix, known as the MakiNakagawa-Sakata (MNS) lepton mixing matrix [14], which can be parameterized in the same way as the CKM (Cabibbo-Kobayashi-Maskawa) matrix for quark mixing:

$$
\left(\begin{array}{c}
\nu_{e} \\
\nu_{\mu} \\
\nu_{\tau}
\end{array}\right)=\left(\begin{array}{ccc}
c_{1} & s_{1} c_{3} & s_{1} s_{3} \\
-s_{1} c_{2} & c_{1} c_{2} c_{3}-s_{2} s_{3} e^{i \delta} & c_{1} c_{2} s_{3}+s_{2} c_{3} e^{i \delta} \\
-s_{1} s_{2} & c_{1} s_{2} s_{3}+c_{2} s_{3} e^{i \delta} & c_{1} s_{2} s_{3}-c_{2} c_{3} e^{i \delta}
\end{array}\right)\left(\begin{array}{c}
\nu_{1} \\
\nu_{2} \\
\nu_{3}
\end{array}\right)
$$

where $c_{i} \equiv \cos \theta_{i}, s_{i} \equiv \sin \theta_{i} ; \theta_{i}$ are the the weak mixing angles and $e^{i \delta}$ is a complex phase, (responsible for $\mathrm{CP}$ violation in the quark sector).

The initial state for $\nu_{\alpha}$ at time, $\mathrm{t}=0$, is then:

$$
\left|\nu_{\alpha}(0)\right\rangle=U_{\alpha i}\left|\nu_{i}\right\rangle
$$

where the Einstein summation convention is assumed. Time evolution of the state is then dependant on its energy eigenvalues. If the neutrino has momentum $\mathrm{p}$, then the energies, $E_{i}$ for the mass eigenstates are given by:

$$
E_{i}^{2}=p^{2}+m_{i}^{2}
$$

where $m_{i}$ are the masses. The initial state then evolves as:

$$
\left|\nu_{\alpha}(t)\right\rangle=U_{\alpha i} e^{-i E_{i} t}\left|\nu_{i}\right\rangle
$$

Now, the probablity of the neutrino being in state $\nu_{\beta}$ at time $\mathrm{t}$ is given by:

$$
\begin{aligned}
P_{\alpha \rightarrow \beta} & =\left|\left\langle\nu_{\beta} \mid \nu_{\alpha}(t)\right\rangle\right|^{2} \\
& =\left|\left\langle U_{\beta i} \nu_{i} \mid U_{\alpha i} e^{-i E_{i} t} \nu_{i}\right\rangle\right|^{2} \\
& =\sum_{i}\left|U_{\alpha i}\right|^{2}\left|U_{\beta i}\right|^{2}+\sum_{i \neq j} U_{\alpha i} U_{\beta i}^{*} U_{\alpha j}^{*} U_{\beta j} e^{-i\left(E_{i}-E_{j}\right) t}
\end{aligned}
$$

Probabilities can also be calculated for Majorana mass terms, however this is more complicated and requires a $6 \times 6$ mixing matrix to account for the fact that neutrinos can also oscillate into anti-neutrinos. 


\section{Two Flavour Mixing}

It is expected that all three neutrino species will mix together, however, experimental observations indicate that there may be dominant oscillation modes for each neutrino flavour. It is therefore informative to calculate two flavour mixing probabilities. Consider flavours $\nu_{e}$ and $\nu_{\mu}$, related to mass eigenstates $\nu_{1}$ and $\nu_{2}$ as follows:

$$
\left(\begin{array}{c}
\nu_{e} \\
\nu_{\mu}
\end{array}\right)=\left(\begin{array}{cc}
\cos \theta & \sin \theta \\
-\sin \theta & \cos \theta
\end{array}\right)\left(\begin{array}{l}
\nu_{1} \\
\nu_{2}
\end{array}\right)
$$

where $\theta$ is the mixing angle between the mass and flavour eigenstates. The probability of a $\nu_{\mu} \rightarrow \nu_{e}$ oscillation can be calculated as before. The time evolving state for $\nu_{\mu}(t)$ is given by:

$$
\left|\nu_{\mu}(t)\right\rangle=\cos \theta e^{-i E_{2} t}\left|\nu_{2}\right\rangle-\sin \theta e^{-i E_{1} t}\left|\nu_{1}\right\rangle
$$

and the oscillation probability to $\nu_{e}$ is:

$$
\begin{aligned}
P_{\nu_{\mu} \rightarrow \nu_{e}} & =\left|\left\langle\nu_{e} \mid \nu_{\mu}(t)\right\rangle\right| \\
& =\sin ^{2} \theta \cos ^{2} \theta\left(2-e^{-i\left(E_{1}-E_{2}\right) t}-e^{i\left(E_{1}-E_{2}\right) t}\right) \\
& =2 \sin ^{2} \theta \cos ^{2} \theta\left(1-\cos \left(E_{1}-E_{2}\right) t\right)
\end{aligned}
$$

Now, $E_{i}^{2}=p^{2}+m_{i}^{2}$ but $m_{i} \ll p$ hence:

$$
\begin{aligned}
E_{i} & =\sqrt{p^{2}+m_{i}^{2}} \\
& \approx p+\frac{m_{i}^{2}}{2 p}
\end{aligned}
$$

and:

$$
\begin{aligned}
E_{1}-E_{2} & \approx \frac{m_{1}^{2}-m_{2}^{2}}{2 p} \\
& \approx \frac{\Delta m_{12}^{2}}{2 p}
\end{aligned}
$$


where $\Delta m_{12}^{2}=m_{1}^{2}-m_{2}^{2}$. Also, the neutrino is highly relativistic so $t \sim L$, where $L$ is distance travelled. Using these results:

$$
P_{\nu_{\mu} \rightarrow \nu_{e}}=\sin ^{2} 2 \theta \sin ^{2}\left(\frac{\Delta m_{12}^{2} L}{4 p}\right)
$$

in natural units. Express energy in $\mathrm{GeV}$, distance in $\mathrm{km}$ and mass in $\mathrm{eV}$ to obtain:

$$
P_{\nu_{\mu} \rightarrow \nu_{e}}=\sin ^{2}(2 \theta) \sin ^{2}\left(\frac{1.27 \Delta m_{12}^{2} L}{E}\right)
$$

the familiar two flavour oscillation relation.

Using the baseline for the MINOS experiment, the oscillation probabilities as a function of neutrino energy are shown in Figure 2.2. Three different values of $\Delta m^{2}$ are used within the region of parameter space suggested by atmospheric neutrino experiments, (assuming $\sin ^{2} 2 \theta=1$ ).

\subsubsection{Matter-Enhanced Oscillations - The MSW Effect}

Oscillation probabilities in the vacuum depend not only on energy, distance and mass differences, but also on the value of the weak mixing angle. For small mixing angles, the oscillation probability will always be small. However, by considering neutrino oscillations in the presence of matter, large probabilities can still be attained. This was first considered by Wolfenstein [15], Mikheyev and Smirnov [16] and is known as the MSW effect.

Neutrinos will interact when propagating through matter. The crosssections are very small $\left(\sim 10^{-42}-10^{-43} \mathrm{~m}^{2}\right)$, but when passing through large quantities of material, such as solar neutrinos travelling through the Sun and perhaps atmospheric neutrinos through the Earth, there may be observable effects. Neutrinos can take part in elastic forward scattering such that their momentum is left unchanged. Figure 2.3 shows the neutral current interactions which all (non-sterile) neutrino flavours undergo. Figure 2.4 shows 


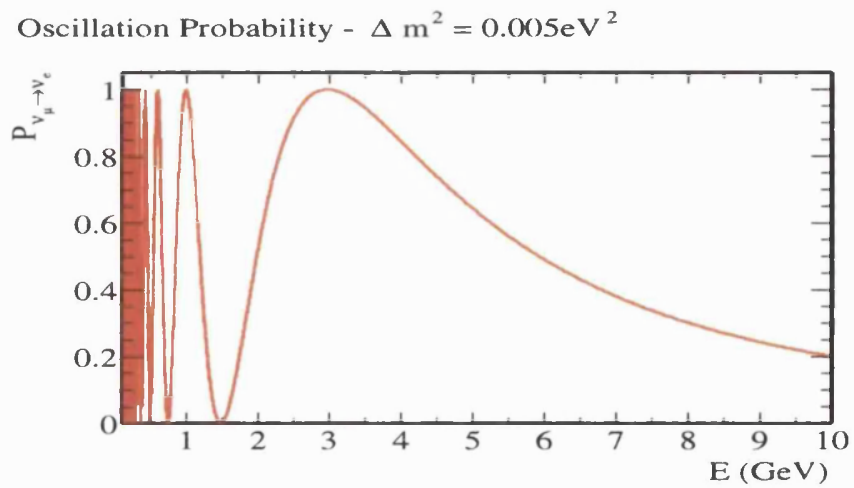

Oscillation Probability $-\Delta \mathrm{m}^{2}=0.002 \mathrm{eV}^{2}$

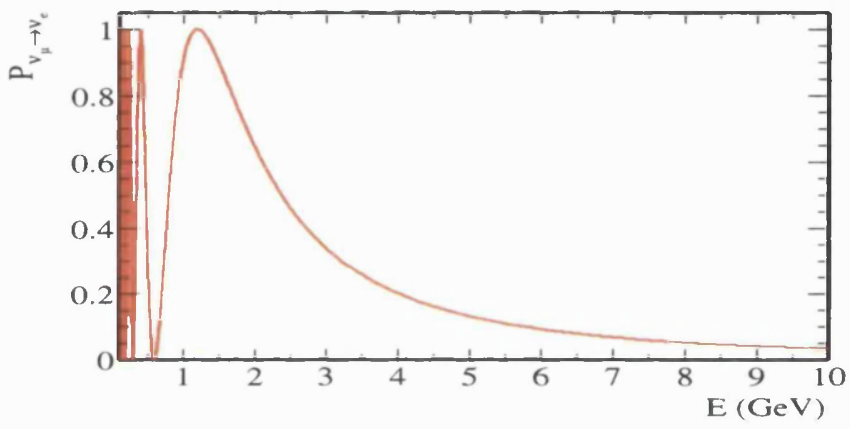

Oscillation Probability $-\Delta \mathrm{m}^{2}=0.001 \mathrm{eV}^{2}$

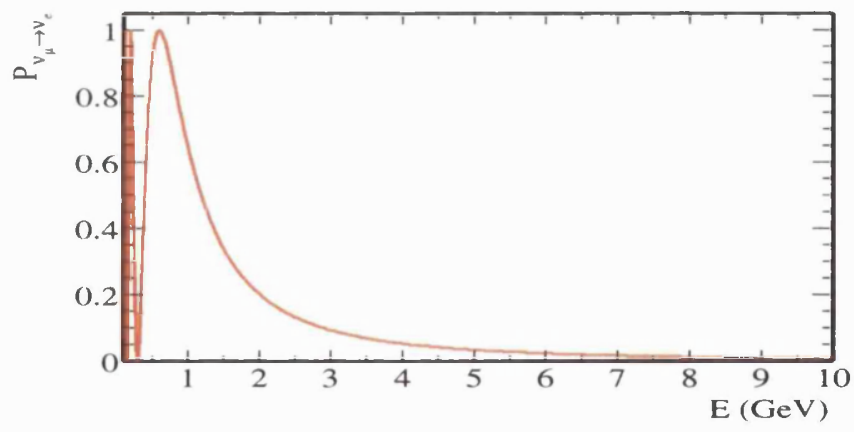

Figure 2.2: Oscillation probability, $P_{\nu_{\mu} \rightarrow \nu_{e}}$, as a function of neutrino energy for the MINOS baseline. Three values of $\Delta m^{2}$ are shown: $0.005 \mathrm{eV}^{2}, 0.002 \mathrm{eV}^{2}$ and $0.001 \mathrm{eV}^{2}$, with $\sin ^{2} 2 \theta=1$ in each case.

an extra charged current interaction only available to electron neutrinos and electron anti-neutrinos.

Having this extra interaction, a potential term is introduced into the 


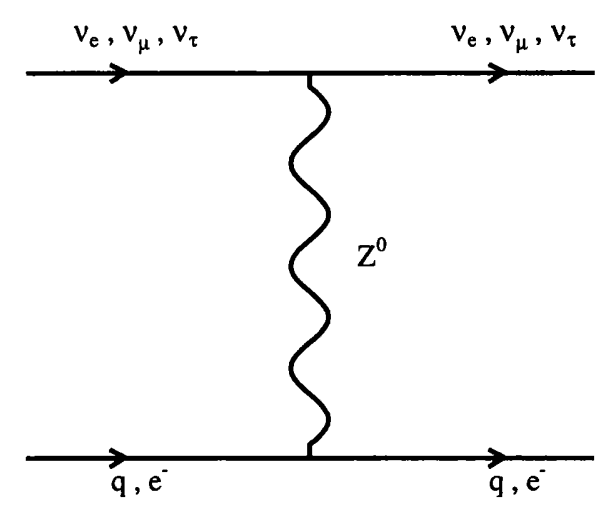

Figure 2.3: Feynman diagram for neutral current neutrino interactions with nucleons (quarks) and electrons in matter. This process is experienced by all non-sterile neutrino flavours.

Hamiltonian of the electron (anti-)neutrino. This term is the result of coherent forward scattering which contributes to the energy of $\nu_{e}$ in matter. The magnitude of this effect depends on the electron number density, $N_{e}$ and is given by:

$$
V_{C C}\left(\nu_{e}\right)=\sqrt{2} G_{F} N_{e}
$$

where $G_{F}$ is the Fermi constant. (For anti-neutrinos, $V_{C C}\left(\bar{\nu}_{e}\right)=-V_{C C}\left(\nu_{e}\right)$ ).

Assuming once again only two flavour mixing, the time evolution of the neutrino states in matter can be derived. In the mass eigenstate basis, the evolution equation is given by:

$$
i \frac{d}{d t}\left(\begin{array}{c}
\nu_{1} \\
\nu_{2}
\end{array}\right)=\left(\begin{array}{cc}
E_{1} & 0 \\
0 & E_{2}
\end{array}\right)\left(\begin{array}{c}
\nu_{1} \\
\nu_{2}
\end{array}\right)
$$

To simplify the derivation, change to the flavour basis:

$$
i \frac{d}{d t}\left(\begin{array}{c}
\nu_{e} \\
\nu_{\mu}
\end{array}\right)=\left(\begin{array}{cc}
E_{1} \cos ^{2} \theta+E_{2} \sin ^{2} \theta & \left(E_{2}-E_{1}\right) \sin \theta \cos \theta \\
\left(E_{2}-E_{1}\right) \sin \theta \cos \theta & -E_{1} \sin ^{2} \theta+E_{2} \cos ^{2} \theta
\end{array}\right)\left(\begin{array}{c}
\nu_{e} \\
\nu_{\mu}
\end{array}\right)
$$


(a)

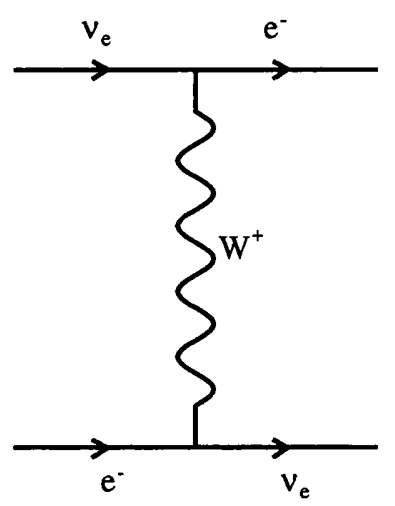

(b)

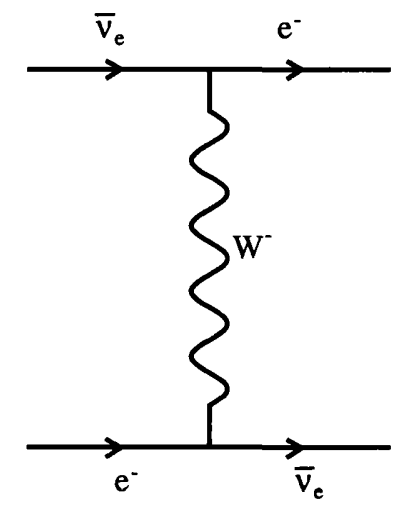

Figure 2.4: Feynman diagrams for (a) charged current electron neutrino and (b) charged current electron anti-neutrino interactions with electrons in matter.

Now add the contribution from the effective potential to the electron neutrino diagonal term:

$i \frac{d}{d t}\left(\begin{array}{c}\nu_{e} \\ \nu_{\mu}\end{array}\right)=\left(\begin{array}{cc}\left(E_{2}-E_{1}\right) \sin ^{2} \theta+\sqrt{2} G_{F} N_{e} & \left(E_{2}-E_{1}\right) \sin \theta \cos \theta \\ \left(E_{2}-E_{1}\right) \sin \theta \cos \theta & \left(E_{2}-E_{1}\right) \cos ^{2} \theta\end{array}\right)\left(\begin{array}{c}\nu_{e} \\ \nu_{\mu}\end{array}\right)$ (Note that $E_{1} I$ has been subtracted from the Hamiltonian. Its contribution to the evolution of the neutrino states is that of a common phase and therefore has no effect on neutrino oscillations which are a consequence of phase differences.)

The Hamiltonian can be diagonalized by choosing the eigenstates:

$$
\begin{aligned}
& \left|\nu_{1}^{\prime}\right\rangle=\cos \theta_{M}\left|\nu_{e}\right\rangle+\sin \theta_{M}\left|\nu_{\mu}\right\rangle \\
& \left|\nu_{2}^{\prime}\right\rangle=-\sin \theta_{M}\left|\nu_{e}\right\rangle+\cos \theta_{M}\left|\nu_{\mu}\right\rangle
\end{aligned}
$$

where the matter mixing angle $\theta_{M}$ is given by:

$$
\tan \left(2 \theta_{M}\right)=\frac{\left(E_{2}-E_{1}\right) \sin 2 \theta}{\left(E_{2}-E_{1}\right) \cos 2 \theta-\sqrt{2} G_{F} N_{e}}
$$


The probability of $\nu_{\mu} \rightarrow \nu_{e}$ oscillations can now be derived as for the vacuum oscillation scenario.

$$
P_{\nu_{\mu} \rightarrow \nu_{e}}=\sin ^{2} 2 \theta_{M} \sin ^{2}\left(\frac{\left(E_{1}^{\prime}-E_{2}^{\prime}\right) L}{2}\right)
$$

where $E_{i}^{\prime}$ are the energy eigenvalues and the difference $E_{1}^{\prime}-E_{2}^{\prime}$ is given by:

$$
E_{1}^{\prime}-E_{2}^{\prime}=\frac{\Delta m_{21}^{2}}{2 E} \sqrt{\sin ^{2} 2 \theta+\left(\cos 2 \theta-\frac{\sqrt{2} G_{F} N_{e}}{\Delta m_{21}^{2} / 2 E}\right)^{2}}
$$

Thus, the form of the oscillation probability in vacuum is recovered:

$$
P_{\nu_{\mu} \rightarrow \nu_{e}}=\sin ^{2} 2 \theta_{M} \sin ^{2}\left(\frac{1.27 \Delta M^{2} L}{E}\right)
$$

where

$$
\Delta M^{2}=\Delta m_{21}^{2} \sqrt{\sin ^{2} 2 \theta+\left(\cos 2 \theta-\frac{\sqrt{2} G_{F} N_{e}}{\Delta m_{21}^{2} / 2 E}\right)^{2}}
$$

At low matter densities, when $N_{e} \sim 0$, then $\Delta M^{2} \sim \Delta m_{21}^{2}$ and $\sin ^{2} 2 \theta_{M} \sim$ $\sin ^{2} 2 \theta$ and the expression for vacuum oscillations is recovered. At high matter densities, $\sin ^{2} 2 \theta_{M} \propto 1 / N_{e}^{2}$ and so oscillations are suppressed by $1 / N_{e}^{2}$.

An important feature of the MSW effect is that when:

$$
\frac{\sqrt{2} G_{F} N_{e}}{\Delta m_{21}^{2} / 2 E}=\cos 2 \theta
$$

the mixing is maximal and a resonant enhancement of the oscillation probability occurs provided $\Delta m_{21}^{2}>0$ for neutrinos (and $\Delta m_{21}^{2}<0$ for antineutrinos). Further, it is interesting to note that resonant enhancement is possible even if the vacuum mixing angle is small. Figure 2.5 shows the value of $\sin ^{2} 2 \theta_{M}$ as a function of $E / \Delta m_{21}^{2}$; Figure 2.6 shows the modified oscillation probability as a function of $E / \Delta M^{2}$ for $\nu_{\mu} \rightarrow \nu_{e}$ in the presence of matter. Three different values for $\theta$, the mixing angle in vacuum, are shown in both figures. 


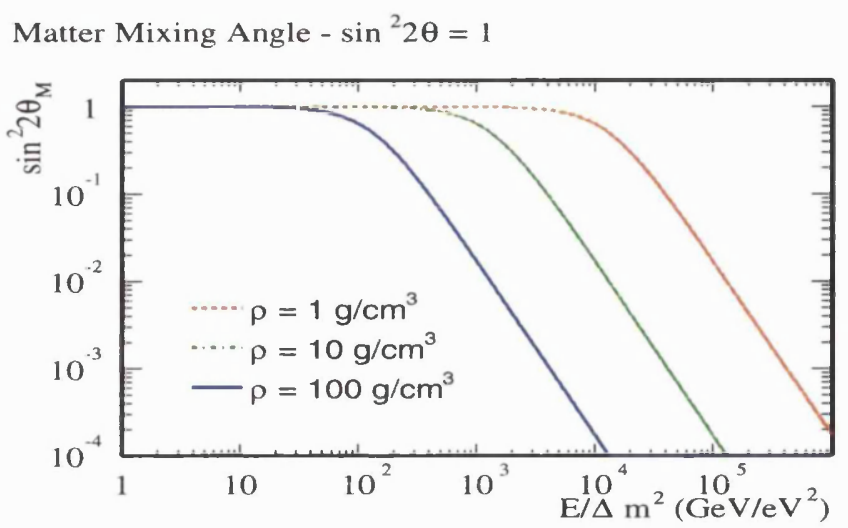

Matter Mixing Angle $-\sin ^{2} 2 \theta=0.1$

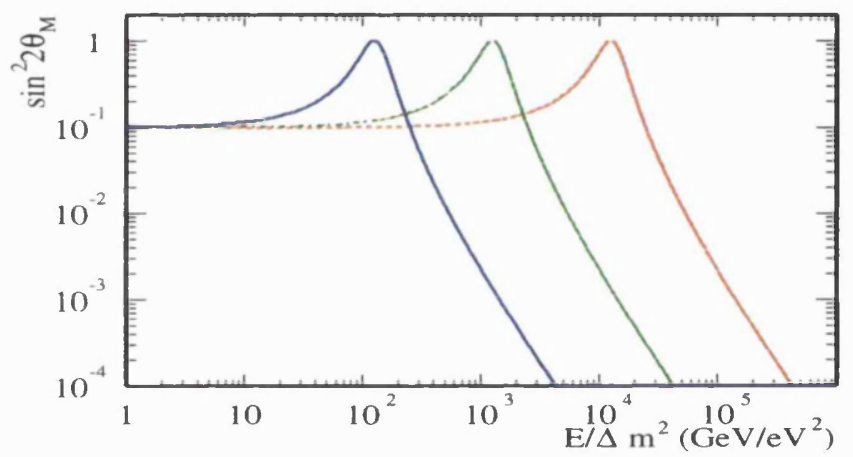

Matter Mixing Angle $-\sin ^{2} 2 \theta=0.01$

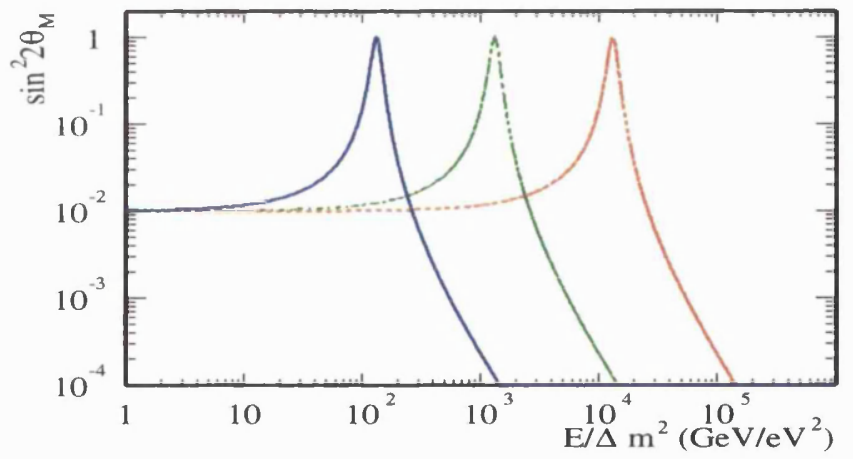

Figure 2.5: Resonant enhancement of neutrino oscillations due to the MSW effect.

The passage of neutrinos through matter can induce a effective maximal mixing of the flavour and mass eigenstates even when mixing in vacuum is not maximal. $\rho$ is the matter density of the material being traversed in units of $\mathrm{g} / \mathrm{cm}^{3}$. 


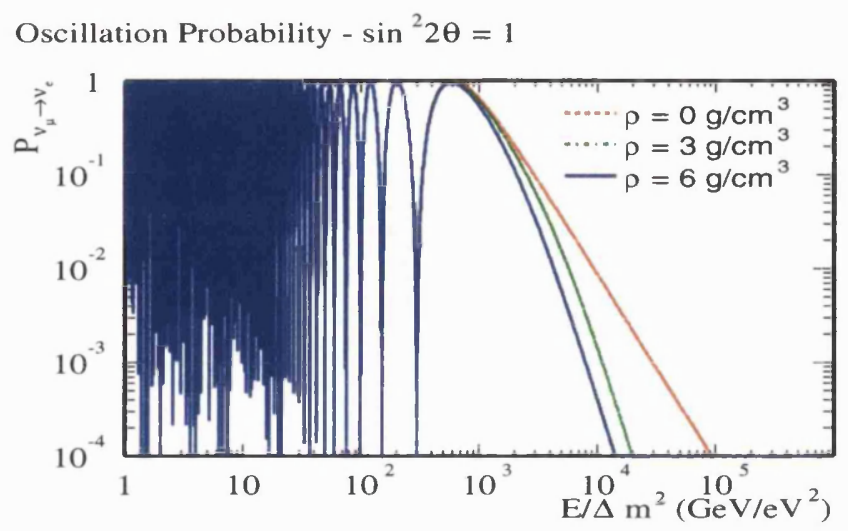

Oscillation Probability $-\sin ^{2} 2 \theta=0.1$

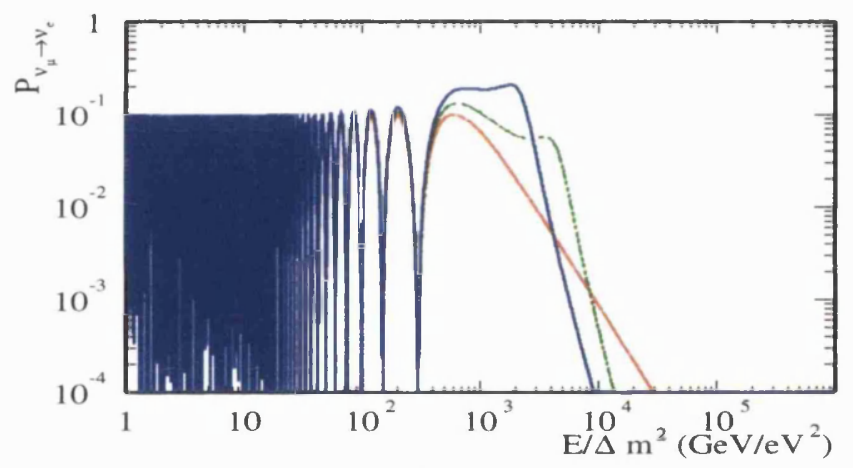

Oscillation Probability $-\sin ^{2} 2 \theta=0.01$

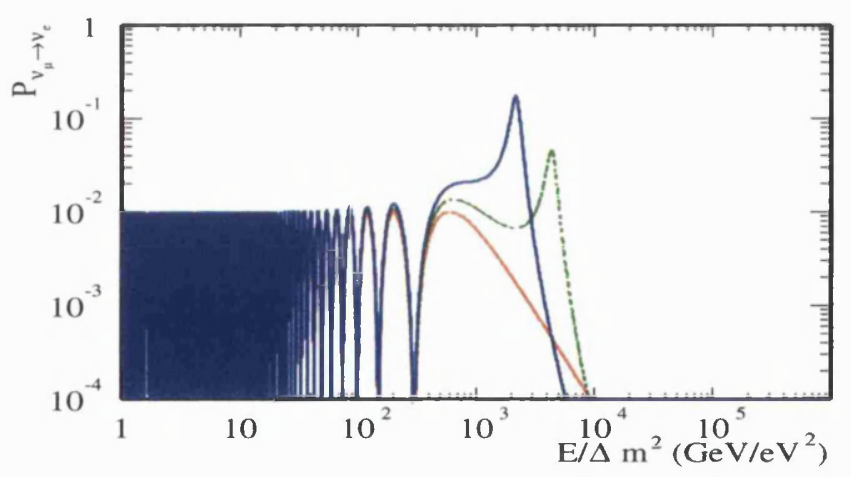

Figure 2.6: $\nu_{\mu} \rightarrow \nu_{e}$ MSW oscillation probability for the MINOS baseline for different values of $\theta$, the mixing angle in vacuum. The different colors show the magnitude of the effect for different matter densities, $\rho$ of the Earths crust. 


\section{Chapter 3}

\section{Experimental Searches for Neutrino Oscillations}

The electron neutrino was first discovered in 1955 [17]. Fifty years on, many questions regarding the properties of these particles still remain unanswered. This chapter will introduce the evidence that led to the postulation of neutrino oscillations and the motivation for building an experiment such as MINOS.

\subsection{Solar Neutrino Experiments}

The first indication that neutrinos show behavior not predicted by the Standard Model came from observations of solar neutrinos in 1968. The Sun is powered by nuclear reactions in the core fusing hydrogen nuclei into helium. These reactions occur via two cycles: the pp cycle and the CNO cycle. In both cases, electron neutrinos are produced when protons are converted to neutrons. The energy of these neutrinos are of the order of $1 \mathrm{MeV}$.

The Homestake chlorine experiment [18] consists of 100,000 gallons of 
tetrachloroethylene which allows the detection of electron neutrinos above an energy threshold of $814 \mathrm{keV}$ via the reaction:

$$
\nu_{e}+{ }^{37} \mathrm{Cl} \rightarrow e^{-}+{ }^{37} \mathrm{Ar}
$$

The neutrinos produced in the reactions of ${ }^{7} \mathrm{Be}$ and ${ }^{8} \mathrm{~B}$ in the pp cycle are principally observed using this technique. The experiment measured the flux of solar electron neutrinos and noticed a deficit compared to that expected by the Standard Solar Model (SSM) [19].

Later experiments, namely SAGE [21][22], GALLEX [23][24], Kamiokande [25] and Super-Kamiokande [2][26][27], have confirmed the results from Homestake. SAGE and GALLEX, (and its successor GNO [28]) are radiochemical experiments employing gallium rather than chlorine. The key reaction is:

$$
\nu_{e}+{ }^{71} G a \rightarrow e^{-}+{ }^{71} G e
$$

which has a threshold energy of $233 \mathrm{keV}$. These experiments are sensitive predominantly to the pp neutrinos produced in the Sun.

The radiochemical experiments infer the flux of the solar neutrinos from integrated running over a period of a few weeks. The elements produced in the reactions are filtered out and counted providing a measurement of the average neutrino flux for that period. An alternative approach, which also provides the advantage of real time measurements, was taken by Kamiokande and its successor Super-Kamiokande.

The Kamiokande detectors are water-Cerenkov detectors located $1000 \mathrm{~m}$ underground in the Kamioka mine in Japan. Relativistic particles in the detector produce Cerenkov light which is then observed by inward facing photomultiplier tubes (PMTs). Kamiokande consisted of $3 \mathrm{kT}$ of purified water and 980 PMTs whereas Super-Kamiokande is a 50kT detector equipped with $\sim 11,000$ PMTs. 
Solar neutrinos are detected through elastic scatters with electrons in the detector. The recoil momentum direction of the electron is correlated with the momentum direction of the incoming neutrino and therefore the Sun's position in the sky. The energy threshold for the experiments is set by the threshold for detecting the recoil electron. This is about 5-7.5 MeV and so these experiments are predominantly sensitive to the ${ }^{8} B$ neutrino flux. Figure 3.1 shows the predicted solar neutrino energy spectrum with the thresholds for the experiments described.

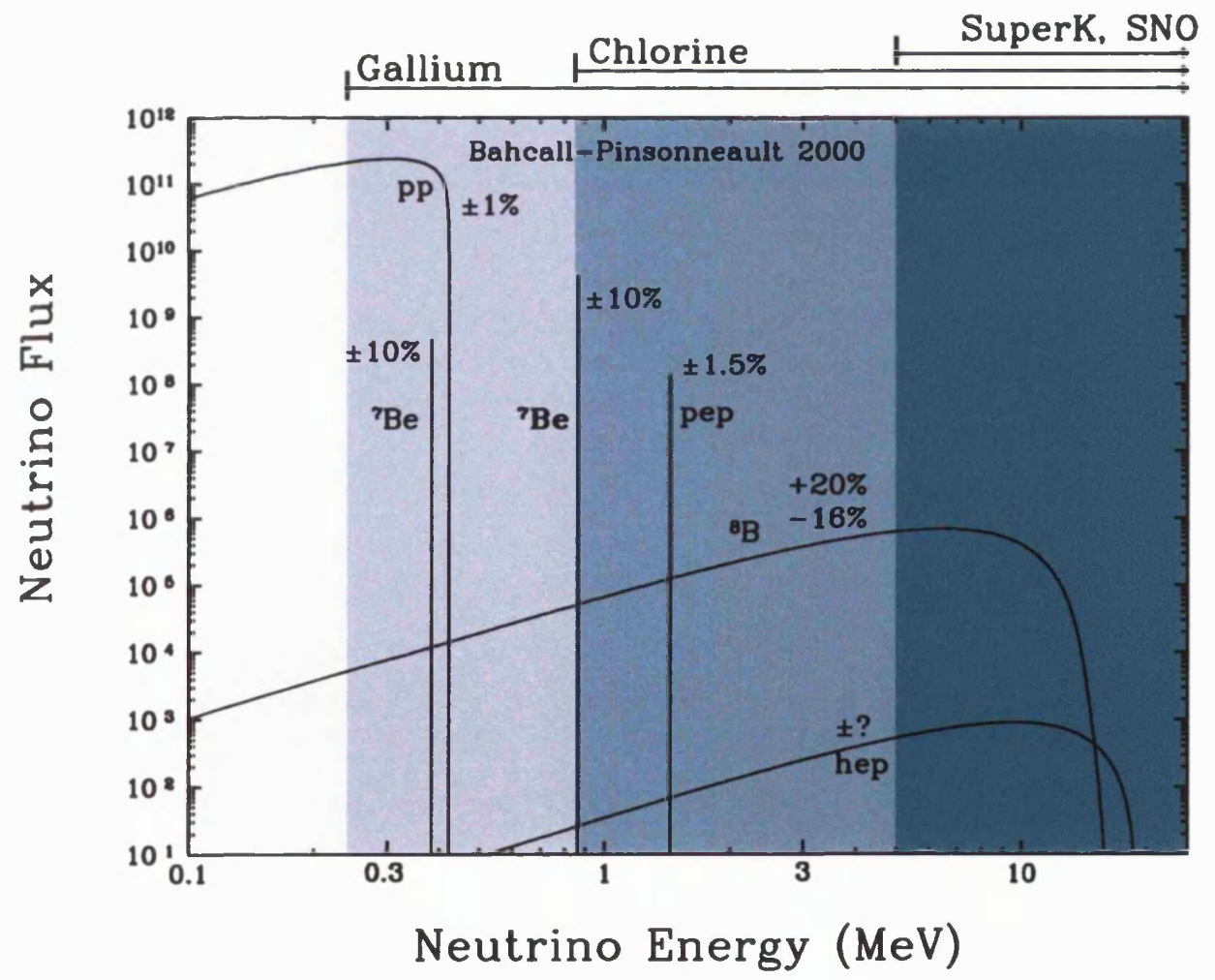

Figure 3.1: The predicted solar neutrino energy spectrum showing the energy thresholds for a number of solar neutrino experiments. From [20]

The results from these solar neutrino experiments are summarized in Fig- 
ure 3.2. All three types of experiments show a deficit in the neutrino flux from the sun which may imply the need for a modification to the SSM. However, looking more closely, it can be seen that there is no consistent way to explain the results purely by altering the SSM. The Kamioka experiments essentially measure the ${ }^{8} B$ neutrino flux, but the flux of the Homestake experiment is smaller despite being sensitive to more of the total neutrino flux. The only way to make the Homestake results consistent with the Kamioka results would be to suppress the ${ }^{7} B e$ neutrinos, except that to form ${ }^{8} B$ in the sun requires the presence of ${ }^{7} \mathrm{Be}$.

Total Rates: Standard Model vs. Experiment Bahcall-Pinsonneault 98

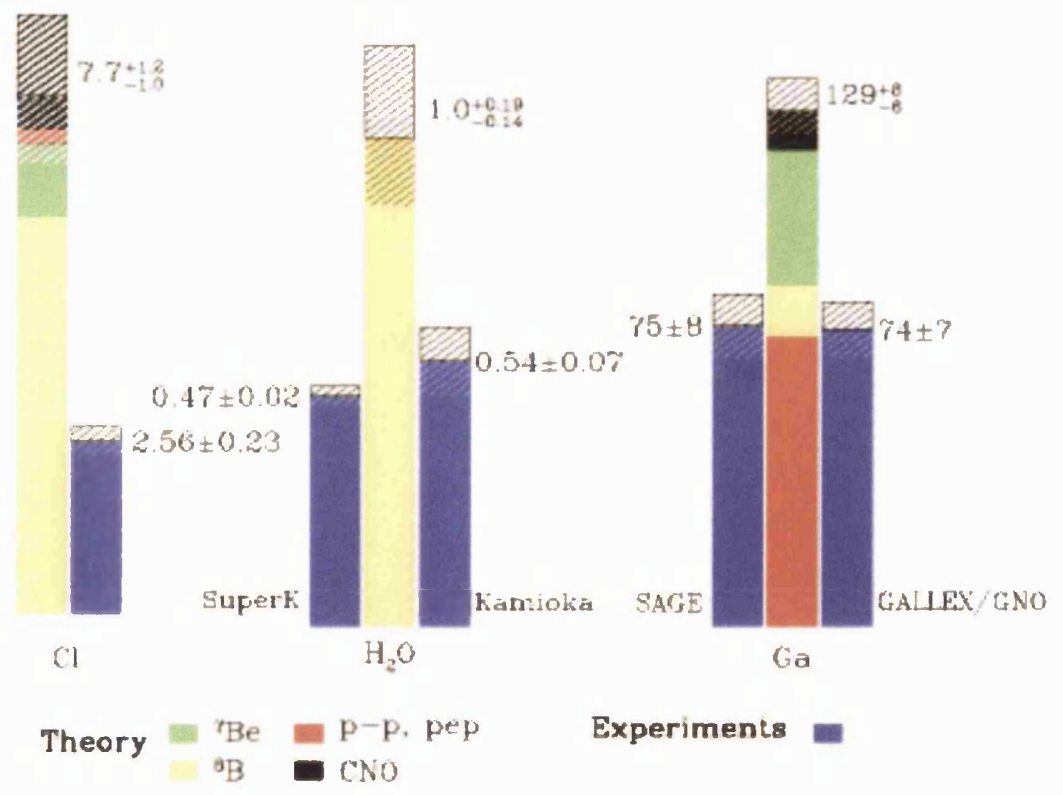

Figure 3.2: Measured neutrino fluxes from a number of solar neutrino experiments. The fluxes predicted by the Standard Solar Model are also shown. From [20]

Further, looking at the gallium experiment results, the total measured 
flux is approximately equal to the expected flux from pp neutrinos. The $\mathrm{pp}$ cycle in the Sun is well understood from luminosity measurements and the pp neutrino flux is known to $1 \%$. Therefore the contributions from ${ }^{7} \mathrm{Be}$, which must be there according to the Homestake measurements, cannot be accommodated. It would therefore appear that the results cannot be understood within the current theoretical framework.

\subsubsection{SNO}

The most recent experiment to focus on the solar neutrino deficit problem is the Sudbury Neutrino Observatory (SNO) [3][4]. The detector is also a water-Cerenkov detector, but uses heavy water $\left(D_{2} O\right)$ rather than normal water $\left(\mathrm{H}_{2} \mathrm{O}\right)$. This difference enables SNO to distinguish electron neutrino charged current $(\mathrm{CC})$ events from neutral current $(\mathrm{NC})$ events (of all flavours).

In normal water, an electron neutrino and an electron can interact via a $\mathrm{W}$ or a $\mathrm{Z}$. However in both cases, the final states are the same and so the processes cannot be distinguished. In heavy water, the interactions with the electrons occur although the cross-section for neutrino interactions with the deuteron dominate. The $\mathrm{CC}$ and $\mathrm{NC}$ interactions with the deuteron have different final state products:

$$
\begin{array}{ll}
\mathrm{CC}: & \nu_{e}+d \rightarrow p+p+e^{-} \\
\mathrm{NC}: & \nu+d \rightarrow n+p+\nu
\end{array}
$$

The CC events produce an electron which emits Cerenkov light and the $\mathrm{NC}$ events produce a neutron, provided the energy transferred is greater than the deuteron binding energy $(2.2 \mathrm{MeV})$. The neutron is then detected by photons produced in neutron capture. In heavy water, this occurs with $\sim 25 \%$ 
efficiency, and so, in a second phase of running, salt $(\mathrm{NaCl})$ is added to improve the efficiency of neutron capture to $\sim 85 \%$. A third phase is also being undertaken to remove the salt and then install ${ }^{3} \mathrm{He}$ proportional counters to directly measure the neutrons ( $\sim 45 \%$ efficiency). This measurement of the neutrino spectrum has completely different systematics and so provides a means to cross-check the results.

Results from the first phase at SNO have measured the ${ }^{8} B$ neutrino flux and shown that there is direct evidence for a non-electron flavour active component in the solar neutrino flux [3]. The measurements also determine the flux of ${ }^{8} B$ active neutrinos to be in close agreement with the predictions of solar models. SNO have also measured day and night solar neutrino energy spectra and rates, but no significant effect has been observed. Using all the SNO results, a global solar neutrino analysis in terms of matter-enhanced oscillations of two active flavours strongly favoured a large mixing angle (LMA) solution with a best fit of $\Delta m^{2}=5.0 \times 10^{-5} \mathrm{eV}^{2}[4]$.

\subsection{Atmospheric Neutrino Experiments}

Primary cosmic rays incident on the upper atmosphere interact with nucleons producing a shower of secondary particles. The secondary particles include many pions which decay:

$$
\begin{aligned}
& \pi^{ \pm} \rightarrow \mu^{ \pm}+\nu_{\mu}\left(\bar{\nu}_{\mu}\right) \\
& \mu^{ \pm} \rightarrow e^{ \pm}+\nu_{e}\left(\bar{\nu}_{e}\right)+\bar{\nu}_{\mu}\left(\nu_{\mu}\right)
\end{aligned}
$$

Therefore, at the Earth's surface the ratio of $\nu_{\mu}$ to $\nu_{e}$ is expected to be 2:1 to first order. Also, geometric considerations lead to the prediction that the neutrino flux should be up-down symmetric; the numbers of upward-and downward-going neutrinos are expected to be the same. 
Experiments studying these neutrinos quote results in terms of a ratio of ratios:

$$
\mathcal{R} \equiv \frac{\mathcal{R}_{D A T A}}{\mathcal{R}_{M C}}=\frac{\left(N_{\mu} / N_{e}\right)_{D A T A}}{\left(N_{\mu} / N_{e}\right)_{M C}}
$$

where $N_{\mu}$ is the number of muon-like events and $N_{e}$ is the number of electronlike events. This ratio is independent of experimental parameters and allows results from different experiments to be directly compared.

The atmospheric neutrino anomaly reflects the experimental results that have measured $\mathcal{R}$ to be less than unity. The first measurement was made by IMB [29][30], a water-Cerenkov experiment located in the Morton mine, Ohio, USA, which measured $\mathcal{R} \sim 0.6$ [30]. Two years later, this result was confirmed by Kamiokande [31].

However, measurements made by two fine-grained iron calorimeter experiments, NUSEX [32][33] and Frejus [34], reported no significant deviation of $\mathcal{R}$ from unity. This lead to the belief that the description of neutrino interactions in iron and water was incomplete. The Soudan 2 experiment [35], also a fine-grained iron calorimeter, resolved the issue: having higher statistics than the other iron experiments, it confirmed the value of $\mathcal{R}$ measured by the water-Cerenkov experiments. The anomalous measurement of NUSEX and Frejus are believed to have been caused by fluctuations in their data. The findings of all these experiments are summarized in Figure 3.3.

The atmospheric neutrino anomaly is believed to be caused by neutrino oscillations. This hypothesis has been most strongly supported by results from the Super-Kamiokande experiment [1], which, in 1998, first claimed evidence for the oscillation of these neutrinos. 


\section{Summary of Atmospheric Neutrino Measurements}

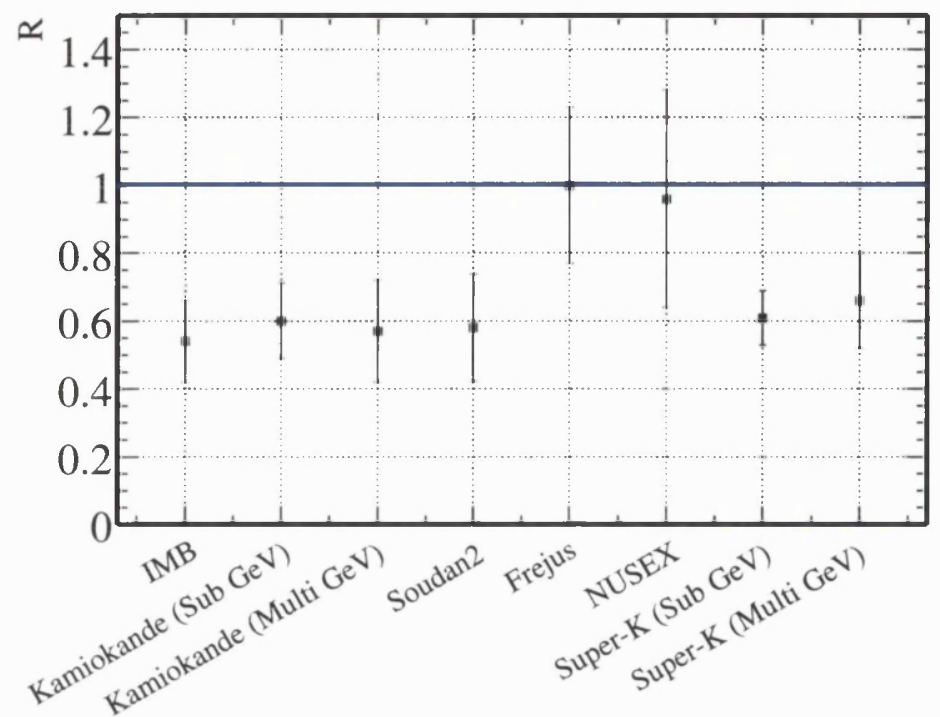

Figure 3.3: Measurements of the ratio of ratios, $\mathcal{R}$ (see text) for a number of solar neutrino experiments.

\subsubsection{Super-Kamiokande}

The Super-Kamiokande detector was briefly described in Section 3.1 as a $50 \mathrm{kT}$ water-Cerenkov detector. Identification and energy measurement of particles produced from CC neutrino interactions with the water nuclei is achieved by measuring the Cerenkov rings generated in the volume. Neutrino interactions in the detector are reconstructed and divided into different categories: Fully contained (FC) events are those in which the neutrino vertex and resulting particle tracks are entirely within the fiducial volume. The FC events are further subdivided into sub-GeV and multi-GeV events, where the energy threshold between the two categories is $1.33 \mathrm{GeV}$.

Partially contained (PC) events are those for which the neutrino vertex is within the volume but a primary particle track, usually a muon, leaves the detector. The final category is upward-going muons (UPMU); for these 
events a neutrino coming from below interacts in the rock and produces a muon which is then detected in the volume. The neutrino samples produced by dividing the events into these categories allow different ranges of neutrino energy to be explored.

Super-K is able to test oscillation hypotheses by measuring the zenith angle of the incident neutrino. This in turn gives the distance the neutrino has traveled, as illustrated in Figure 3.4. In 1998, Super-K published a figure for $\mathcal{R}$ of about 0.6 [36], consistent with previous experiments, and went on to claim discovery of neutrino oscillations. They reported an updown asymmetry for muon-like events which varies with zenith angle in a manner consistent with oscillations. The $\nu_{e}$ spectrum showed no distortions and so conclude that the oscillations must be $\nu_{\mu} \rightarrow \nu_{\tau}$ or $\nu_{\mu} \rightarrow \nu_{\text {sterile. }}$.

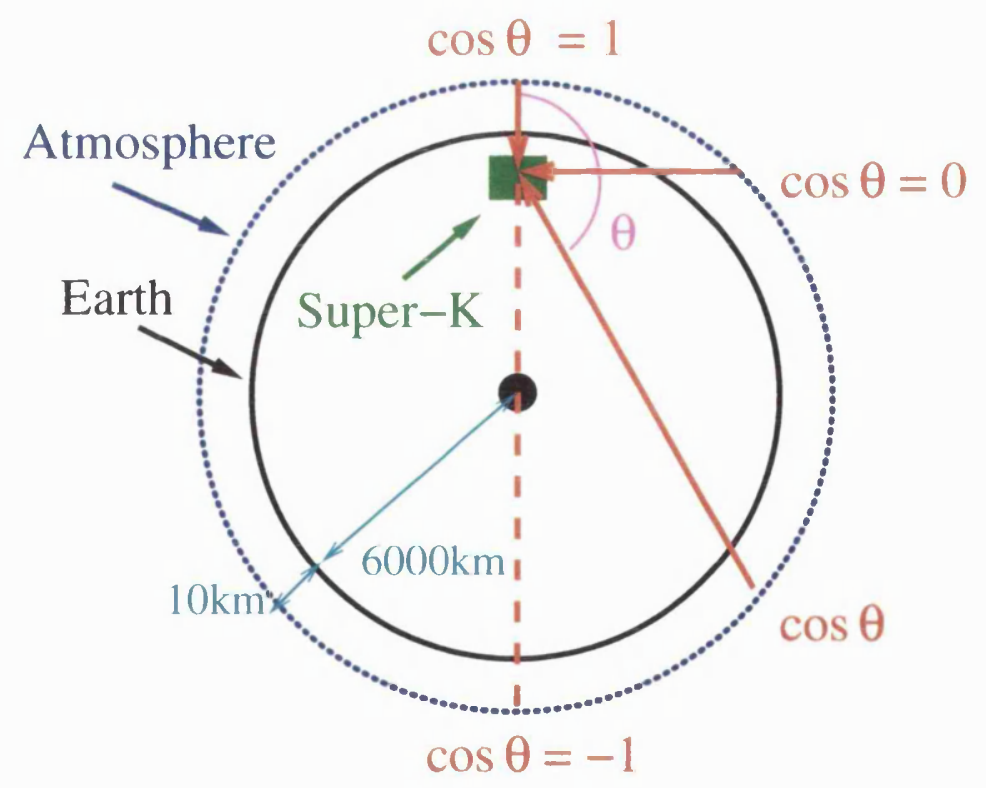

Figure 3.4: A sketch illustrating the variations in distance traveled by atmospheric neutrinos with zenith angle. Super-Kamiokande can use this dependence to test oscillation hypotheses.

With more data, oscillations to purely $\nu_{\text {sterile }}$ have been disfavoured at 
99\% C.L. and interpreting the results in a two flavour scheme where oscillations are $\nu_{\mu} \rightarrow \nu_{\tau}$ the best fit corresponds to maximal mixing and $\Delta m^{2}=2.5 \times 10^{-3} \mathrm{eV}^{2}[37]$.

\subsection{Reactor Neutrino Experiments}

Nuclear reactors employ the elements ${ }^{235} \mathrm{U},{ }^{238} \mathrm{U},{ }^{239} \mathrm{P}$ and ${ }^{241} \mathrm{Pu}$ which, during fission, produce $\bar{\nu}_{e}$ with a mean energy of $\sim 3 \mathrm{MeV}$. Experiments are constructed to detect these neutrinos based on the reaction $\bar{\nu}_{e}+p \rightarrow e^{+}+n$. The detectors are typically located from 10's of metres up to $1 \mathrm{~km}$ from source and search for the disappearance of $\bar{\nu}_{e}$. This gives sensitivity to oscillations with $\Delta m^{2}$ down to $\sim 10^{-3} \mathrm{eV}^{2}$.

The most sensitive reactor experiment is the CHOOZ experiment [38] located $\sim 1 \mathrm{~km}$ from the reactor core of the Chooz power station. The experiment finished in 1998 and after an analysis of the full data set, no evidence for spectral distortion was found. A region of oscillation parameter space has been excluded which indicates that $\nu_{\mu} \rightarrow \nu_{e}$ mixing cannot be the dominant mode for atmospheric region of interest, (at $90 \%$ C.L., $\Delta m^{2}<7 \times 10^{-4} \mathrm{eV}^{2}$ for maximal mixing and $\sin ^{2} 2 \theta<0.10$ for large $\Delta m^{2}$ ). These results corroborate the Super-K interpretation that $\nu_{\mu}$ disappearance and not $\nu_{e}$ appearance is occurring for atmospheric neutrino.

\subsection{Accelerator Neutrino Experiments}

Accelerator neutrino experiments fire high energy particle beams at targets producing large numbers of secondary pions and kaons. These secondaries decay predominantly to muons and muon neutrinos. The advantage of 
this type of neutrino oscillation experiments is that, unlike for atmospheric neutrino experiments, the energies and flavour content of the resulting neutrinos are very well known allowing accurate measurements of the oscillation parameters to be made.

\subsubsection{Short Baseline Experiments}

Short baseline experiments are typically situated approximately $1 \mathrm{~km}$ away from an accelerator source which produces neutrinos at energies ranging from $10^{-2} \mathrm{MeV}$ to $10 \mathrm{GeV}$. Such experiments have sensitivity to oscillations with $\Delta m^{2}$ down to $\sim 0.1 \mathrm{eV}^{2}$ by looking for the appearance of $\nu_{e}$ or $\nu_{\tau}$ from a beam of $\nu_{\mu}$.

At CERN, the NOMAD [39] and CHORUS [40] experiments both looked for $\nu_{\tau}$ appearance. However in both cases no evidence for oscillations was found, excluding $\Delta \mathrm{m}^{2}>1 \mathrm{eV} \mathrm{V}^{2}$ for $\nu_{\mu} \rightarrow \nu_{\tau}$ mixing. The only accelerator experiment thus far to report a positive oscillation signal is the Liquid Scintillator Neutrino Detector (LSND) [41] at the Los Alamos Meson Physics Facility.

\section{LSND}

An intense $798 \mathrm{MeV}$ proton beam interacting in a primary target (which initially was a water target later upgraded to tungsten) was used to produce mostly $\pi^{+}$and a small fraction of $\pi^{-}$. LSND used a neutrino beam produced from pion decay in flight (DIF): $\pi^{+} \rightarrow \mu^{+}+\nu_{\mu}$; and an anti-neutrino beam produced from $\mu^{+}$decay at rest (DAR): $\mu^{+} \rightarrow e^{+}+\nu_{e}+\bar{\nu}_{\mu}$.

Identification of electron anti-neutrino events was achieved via the interaction: $\bar{\nu}_{e}+p \rightarrow e^{+}+n$ followed by neutron capture producing a photon of $2.2 \mathrm{MeV}: n+p \rightarrow d+\gamma$. Identification of electron neutrino events was 
via the interaction: $\nu_{e}+C \rightarrow e^{-}+X$. The electron energies used in the DAR analysis (looking for $\bar{\nu}_{\mu} \rightarrow \bar{\nu}_{e}$ oscillations) ranged from $20-60 \mathrm{MeV}$ while for the DIF analysis ( $\nu_{\mu} \rightarrow \nu_{e}$ oscillations) $60-200 \mathrm{MeV}$ neutrinos were used. The experiment reported an excess of events in both the DAR and DIF beams corresponding to oscillations with $\Delta m^{2} \sim 1 \mathrm{eV}^{2}$ and $\sin ^{2} 2 \theta \sim 10^{-2}$. The allowed parameter space is shown in Figure 3.5.

Figure 3.5 also shows results from other short baseline experiments searching for oscillations in this region. One such is the KARMEN experiment [42] located at the Rutherford Laboratory in the UK which also looks for $\nu_{e}$ appearance from a $\nu_{\mu}$ beam. KARMEN is a liquid scintillator detector which is located $17.5 \mathrm{~m}$ from the neutrino source, (half the LSND baseline). KARMEN measures event rates consistent with expected background and excludes much of the allowed LSND region.

The MiniBooNE [44] experiment at Fermilab, which started taking data in 2002, has been designed to search for oscillations in the region suggested by LSND in an attempt to confirm the findings. Should oscillations occur in the region suggested by LSND, MiniBooNE will observe an 8-10 $\sigma$ signal after one year of running. If the LSND result is anomalous, in two years running the entire LSND region can be excluded at the $90 \%$ C.L.

\subsubsection{Long Baseline Experiments}

The first long baseline experiment built was K2K (KEK-to-Kamioka) [45] which fires an almost pure beam of $\nu_{\mu}$ from KEK to Super-Kamiokande, 250 $\mathrm{km}$ away. The experiment looks for $\nu_{\mu}$ disappearance between the near detector, located at KEK, and the far detector in the Kamioka mine. The average energy of the neutrino beam is $\sim 1.3 \mathrm{GeV}$, hence $\nu_{\tau}$ appearance measurements are not possible. Initial results from $\mathrm{K} 2 \mathrm{~K}$ indicate $\nu_{\mu} \rightarrow \nu_{\tau}$ oscillations in 


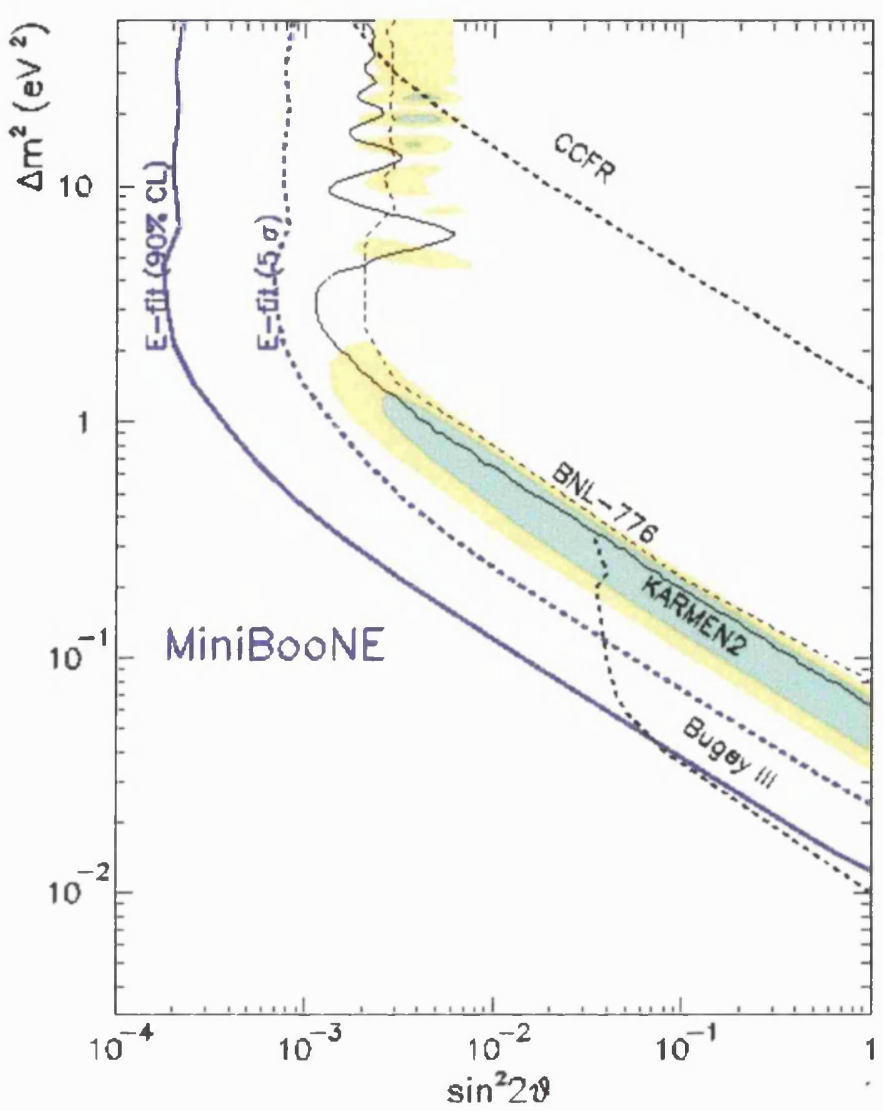

Figure 3.5: The allowed parameter space for the LSND results. Excluded regions from other short baseline experiments are also shown, as well as the expected limits for the MiniBooNE experiment after 2 years. From [43].

the region suggested by Super-Kamiokande, however no interpretation of the results has yet been reported.

Two other long baseline experiments are also expected to begin taking data in the next few years: The CERN-to-Gran Sasso neutrino beam (NCGS) proposes to fire a beam of $\nu_{\mu}$ towards the OPERA emulsion based detector and the ICARUS liquid argon TPC [46]. The detectors are designed to look for $\nu_{\tau}$ appearance in the region suggested by atmospheric neutrino experi- 
ments. The second forthcoming long baseline experiment is MINOS. MINOS also aims to address the atmospheric neutrino anomaly and is explained in detail in Chapter 4.

\subsection{Summary}

Assuming that all the experimental results described above are a consequence of neutrino oscillations, current understanding can be summarized as follows:

- The solar neutrino anomaly suggests $\nu_{e} \rightarrow \nu_{x}$ large mixing for $\Delta m^{2} \sim$ $10^{-5} \mathrm{eV}^{2}$ given MSW oscillation enhancement in the Sun.

- The atmospheric neutrino anomaly suggests $\nu_{\mu} \rightarrow \nu_{x}$ oscillations with maximal mixing and $\Delta m^{2} \sim 10^{-3} \mathrm{eV}^{2}$. Oscillations to $\nu_{e}$ are ruled out by Super-K and CHOOZ. Super-K also excludes $\nu_{\mu} \rightarrow \nu_{\text {sterile }}$ at $99 \%$ C.L.

- LSND results indicate $\nu_{\mu} \rightarrow \nu_{e}$ mixing for $\Delta m^{2} \sim 1 \mathrm{eV}^{2}$.

This information can also be displayed by considering allowed and excluded regions of the neutrino oscillation parameter space assuming that specific oscillations dominate. Figure 3.6 shows the parameter space for $\nu_{\mu} \rightarrow \nu_{\tau}$ oscillations and Figure 3.7 is for $\nu_{\mu} \rightarrow \nu_{e}$ oscillations.

Since $\Delta m_{\text {solar }}^{2}<<\Delta m_{\text {atmospheric }}^{2}<\Delta m_{L S N D}^{2}$ it would appear impossible to describe the current results in a three neutrino framework. Further, results from LEP have shown that there are only 3 active neutrinos [47], implying that a fourth neutrino species would have to be sterile. The current position regarding neutrino oscillations is therefore unclear, and it is essential to obtain more experimental data. Along these lines, MINOS will be able 


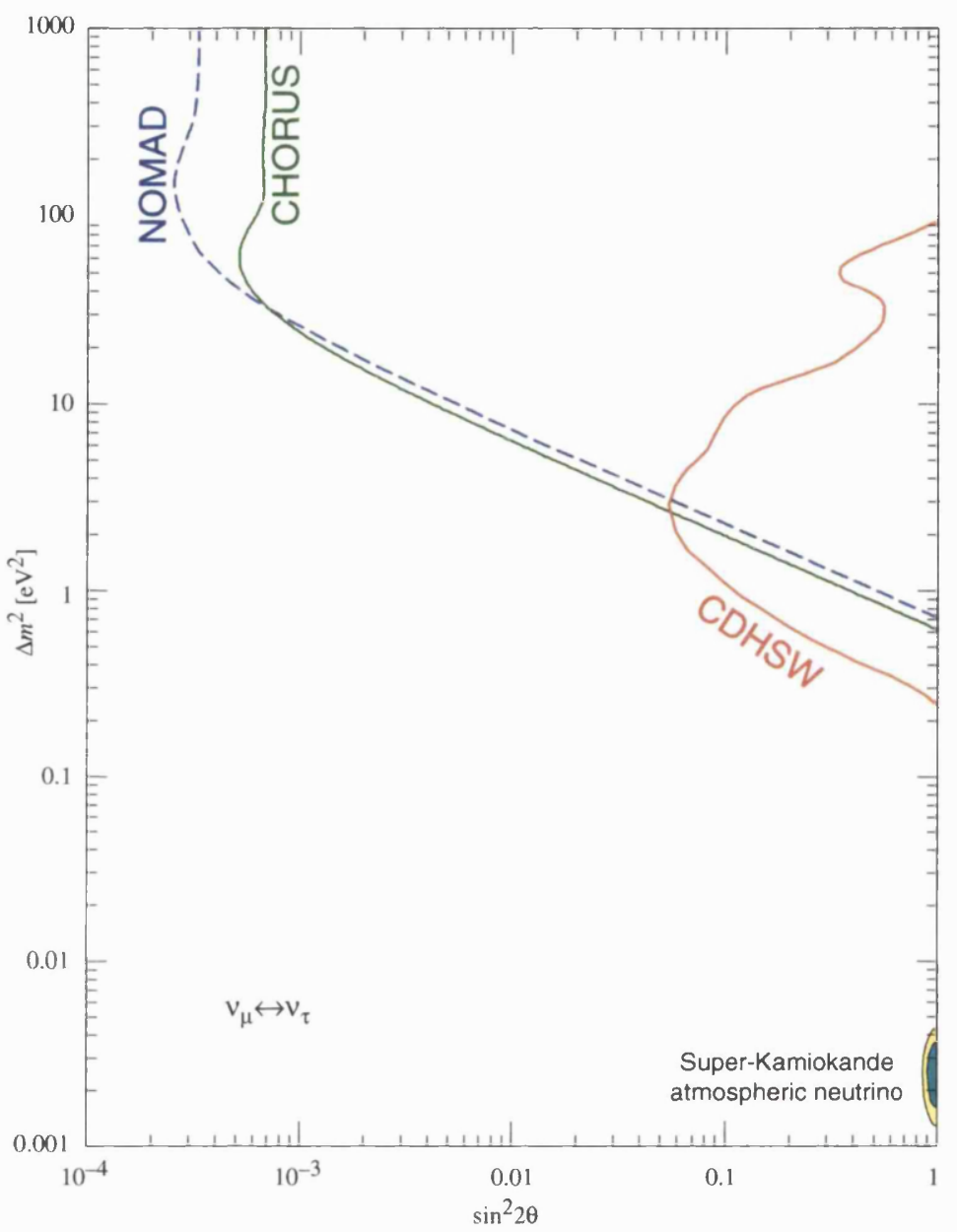

Figure 3.6: Allowed and excluded regions of parameter space for $\nu_{\mu} \rightarrow \nu_{\tau}$ oscillations. Regions excluded by Nomad, Chorus and CDHSW, (short baseline experiments carried out at CERN), are shown along with the allowed regions from Super-Kamiokande. From [9]

to measure whether the atmospheric neutrino anomaly is due to oscillations to $\nu_{\tau}$ or to $\nu_{\text {sterile }}$ and will be able to measure the oscillation parameters to approximately $10 \%$. MiniBooNE will attempt to clarify the LSND effect and SNO will continue to study solar neutrinos. The next few years should produce new results which will shed light on the current confusion in the neutrino sector. 


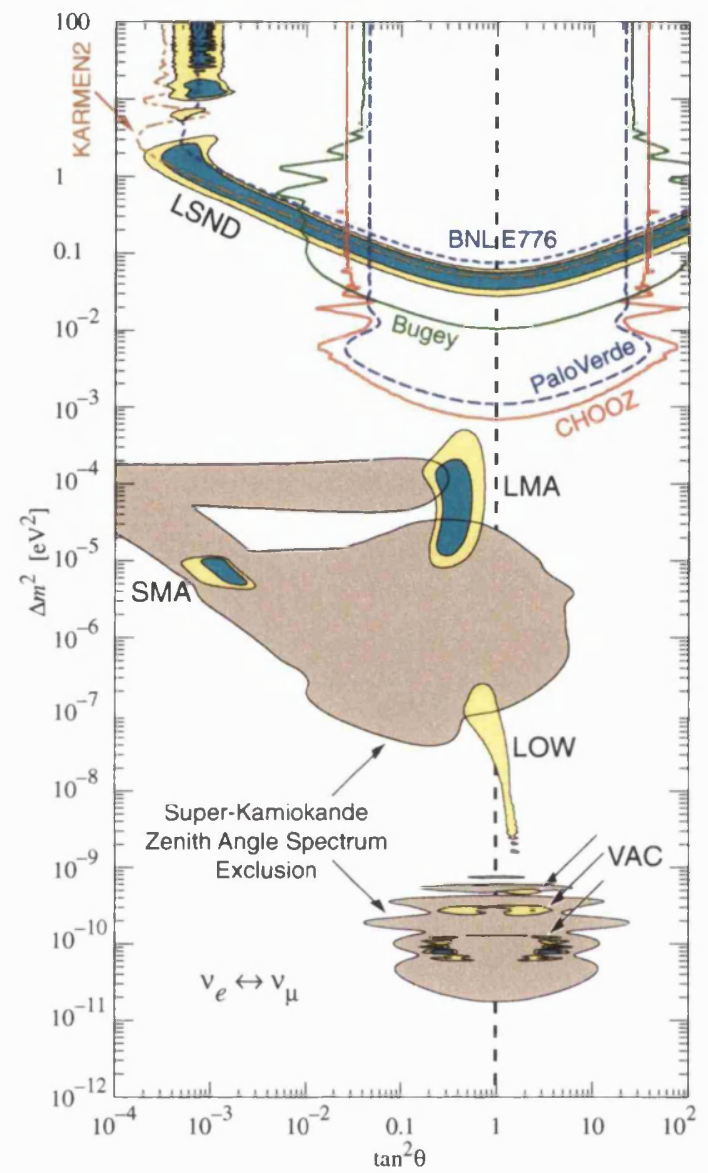

Figure 3.7: Allowed and excluded regions of parameter space for $\nu_{\mu} \rightarrow \nu_{e}$ oscillations. Shown: allowed region for LSND and excluded regions from other short baseline experiments, (Karmen2, Bugey, BNL E776); excluded regions from reactor experiments, (CHOOZ, Palo Verde); preferred solutions from solar neutrino experiments, (based on results from Homestake, SAGE, GALLEX, GNO, Super$\mathrm{K}, \mathrm{SNO}$ ); excluded regions from Super-K zenith angle measurements. The solar neutrino preferred regions are labelled LMA (large mixing angle), SMA (small mixing angle), LOW (low $\Delta m^{2}$ )and VAC (vacuum "Just-so" solution). From [9] 


\section{Chapter 4}

\section{The NuMI-MINOS Experiment}

The NuMI-MINOS experiment is a two detector, long baseline neutrino oscillation search. A beam of mainly muon neutrinos are created at Fermilab using protons from the Main Injector and are fired through the Earth towards the Soudan mine in Northern Minnesota. The beam is first sampled by the Near Detector, on-site at Fermilab, before oscillations have occurred and is then sampled again, $735 \mathrm{~km}$ away, by the Far Detector located in the mine.

This chapter describes the three major components of the NuMI-MINOS experiment: the Beam, the Near Detector and the Far Detector. The physics capabilities of the experiment are also outlined. For more detailed information consult [48] and [49].

\subsection{The Beam}

The NuMI (Neutrinos at the Main Injector) neutrino beam will be the most intense source of neutrinos in the world and is the reason that this type of experiment can take place. The neutrino beam will contain predominantly $\nu_{\mu}$ resulting from the decay of $\pi$ 's and $K$ 's produced when $120 \mathrm{GeV}$ protons 
from the Main Injector are brought to focus on a carbon target. The Main Injector is initially expected to deliver $2.5 \times 10^{13}$ protons on target per spill. The spill is delivered to the target via single-turn extraction to produce a spill lasting $10 \mu \mathrm{s}$.

A diagram of the beam line is shown in Figure 4.1. Two parabolic magnetic horns are used to focus the charged particles produced when the protons interact in the target. The hadrons then pass through a $675 \mathrm{~m}$ long evacuated pipe, where many decay to produce anti-muons and muon neutrinos, (decays of anti-muons to positrons and electron neutrinos occur approximately $1 \%$ of the time). A section of absorber and rock further downstream is used to stop any hadrons that have not decayed. Most of the muons also range out in the $240 \mathrm{~m}$ of rock between the decay pipe and the Near Detector.

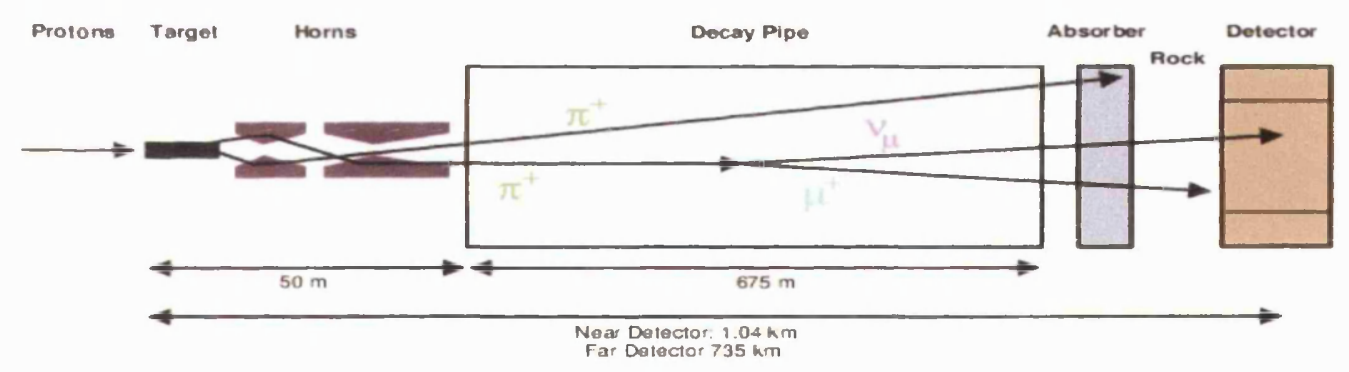

Figure 4.1: The NuMI beamline. Protons from the Main Injector hit the carbon target producing secondary hadrons which are focussed by magnetic horns. The secondary hadrons then decay to produce muons and muon neutrinos. A section of absorber and rock remove hadrons and muons leaving a neutrino beam which intercepts the Near Detector $\sim 1 \mathrm{~km}$ downstream of the target.

Both the magnetic horns and the target are movable. Changing the relative positions allows a beam with one of three different energy spectra to be produced: so called Low, Medium and High energy. The three $\nu_{\mu} \mathrm{CC}$ energy spectra (without oscillations) at the Far Detector associated with the 
three beams are shown in Figure 4.2 along with a schematic diagram of the positions of the horns and target for each case.
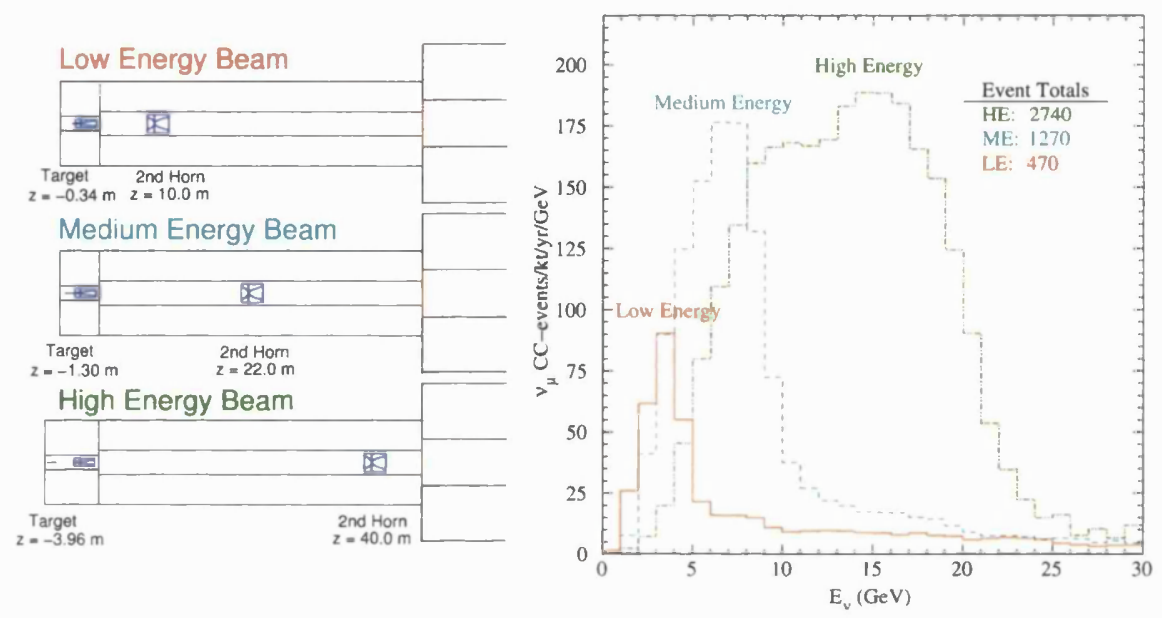

Figure 4.2: The expected Far Detector $\nu_{\mu}$ CC energy spectra for the Low, Medium and High energy neutrino beams, (assuming no oscillations). These are achieved by changing the relative positions of the magnetic horns and target, shown schematically on the left hand side.

The experiment will initially run with the Low energy beam in order to maximize sensitivity to the regions of parameter space suggested by other experiments (see Chapter 3). In this configuration, the neutrino energy spectrum will peak at around $3 \mathrm{GeV}$ at the Far Detector and, without oscillations, the expected $\nu_{\mu}$ CC event rate is $\sim 2400$ per year.

One of the challenges that still remains for MINOS is how to compare the spectra at the Near and Far Detectors. The problem is that as the beam propagates to the Far Detector it diverges such that the neutrino flux at the two locations is different. The beam characteristics can be understood from the decay kinematics of the pions and kaons, however, there is uncertainty regarding the initial hadron content and kinematics. To understand this, a hadron production experiment at Fermilab, MIPP (Main Injector Particle 
Production), will accurately measure the particles produced when protons are incident on the NuMI carbon target. The results of this experiment will then be incorporated into a Monte Carlo simulation to predict the Near/Far neutrino flux differences.

\subsection{The Detectors}

The detectors have been designed to be as similar as possible to reduce systematic errors. It is therefore convenient to describe the main features of the MINOS detectors before highlighting the differences between them. It should also be pointed out at this point that there is a third MINOS detector that has been constructed for calibration purposes. It is very similar in design to the larger detectors and is the subject of Chapter 6 .

\subsubsection{MINOS Detector Technologies}

The MINOS detectors are fine grained tracking calorimeters with an absorber layer of steel and an active layer of plastic scintillator. The scintillator is made of polystyrene, infused with the fluor PPO (1\%) and the wavelength shifter POPOP $(0.030 \%)$. The thickness of the layers is $2.54 \mathrm{~cm}$ and $1 \mathrm{~cm}$ for the steel and scintillator respectively. Each plane of the detector is a sandwich of steel, air and scintillator with a separation between each plane of approximately $6 \mathrm{~cm}$. The scintillator layers are constructed from strips of width $4.1 \mathrm{~cm}$ which, in the case of the Far Detector, can be up to $8 \mathrm{~m}$ long. The orientation of the strips alternates with each successive plane by $\pm 90^{\circ}$. This gives a detector resolution of $\sim 23 \% / \sqrt{E}$ for electromagnetic showers and $\sim 55 \% / \sqrt{E}$ for hadronic showers, where $\mathrm{E}$ is measured in $\mathrm{GeV}$.

Each strip has a groove cut into its wider edge which runs along the length 
of the strip. Kuraray Y11 wavelength shifting (WLS) fibre of diameter 1.2mm is glued into this groove in order to transport light produced in the scintillator to the photodetectors. The strip is also covered in a reflective coating of polystyrene mixed with $\mathrm{TiO}_{2}$ to increase the amount of light captured by the fibre. Figure 4.3 is a photograph of a length of MINOS scintillator.

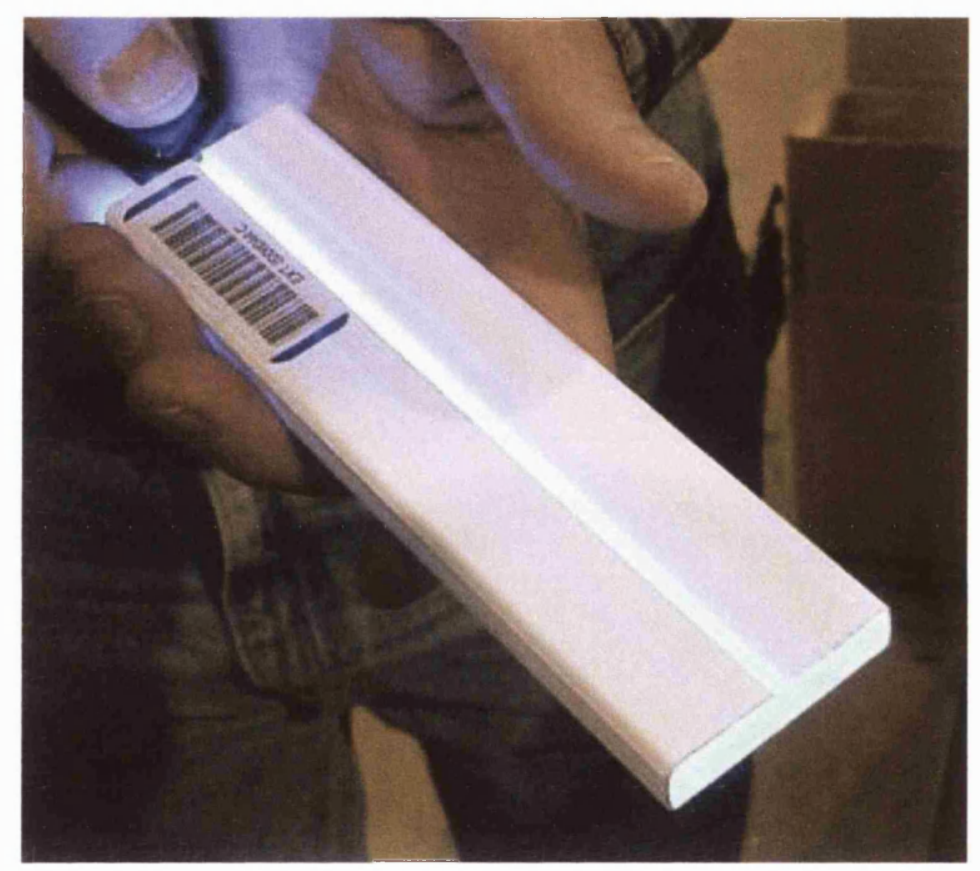

Figure 4.3: A short length of MINOS scintillator being illuminated by a blue LED. The groove for the wavelength shifting (WLS) fibre can clearly be seen along the length of the strip. The strip is coated in a reflective layer to maximize light collection by the WLS fibre.

The scintillator strips are arranged in groups of 20 or 28 and are affixed within a thin layer of aluminium to form modules. These modules also encase plastic manifolds which rout the WLS fibres from each strip-end to an optical connector. Clear fibre is then used to transport the light from the modules to Hamamatsu multipixel phototubes.

The detectors are both magnetized with a current carrying coil producing 
a field of, on average, $1.5 \mathrm{~T}$ in the fiducial volume. This allows the momentum of high energy muons to be measured from curvature, although the resolution is limited by Coulomb scattering in the steel; at $10 \mathrm{GeV}$ the muon momentum can be measured to $\sim 14 \%$ [50]. Low energy stopping muons can be measured more accurately by range. A resolution of approximately $6 \%$ is obtained with this method [50].

\subsubsection{The Far Detector}

The MINOS Far Detector is located in the Soudan Underground Laboratory, Northern Minnesota, at a depth of 2100 metres of water equivalent (m.w.e.). Everything required for the construction, including the detector components and machinery, must be transported down a small mine shaft in parts and reassembled underground.

The Far Detector is the largest of the MINOS detectors at $5.4 \mathrm{kT}$. It is made up from 486 octagonal planes of steel and scintillator each with a diameter of $8 \mathrm{~m}$. The detector is split up into 2 supermodules each with 243 planes. The supermodules are both independently fitted with a $15 \mathrm{kA}$-turn coil which runs along the axis of the supermodule, through the centre of each plane, to provide the magnetic field in the iron. Figure 4.4 is a photograph of the MINOS cavern showing the Far Detector during construction.

Each scintillator plane is constructed from 192 strips arranged in 8 scintillator modules. The strips are read out on both sides of the detector by Hamamatsu M16 multi-pixel phototubes (16 pixels per tube). The signals are multiplexed such that 8 fibres, corresponding to strips separated by approximately $1 \mathrm{~m}$ in the plane, are readout by a single phototube pixel. Demultiplexing is achieved by permuting the local order of the fibres on one side of the detector with respect to the other. Thus, each side produces a 


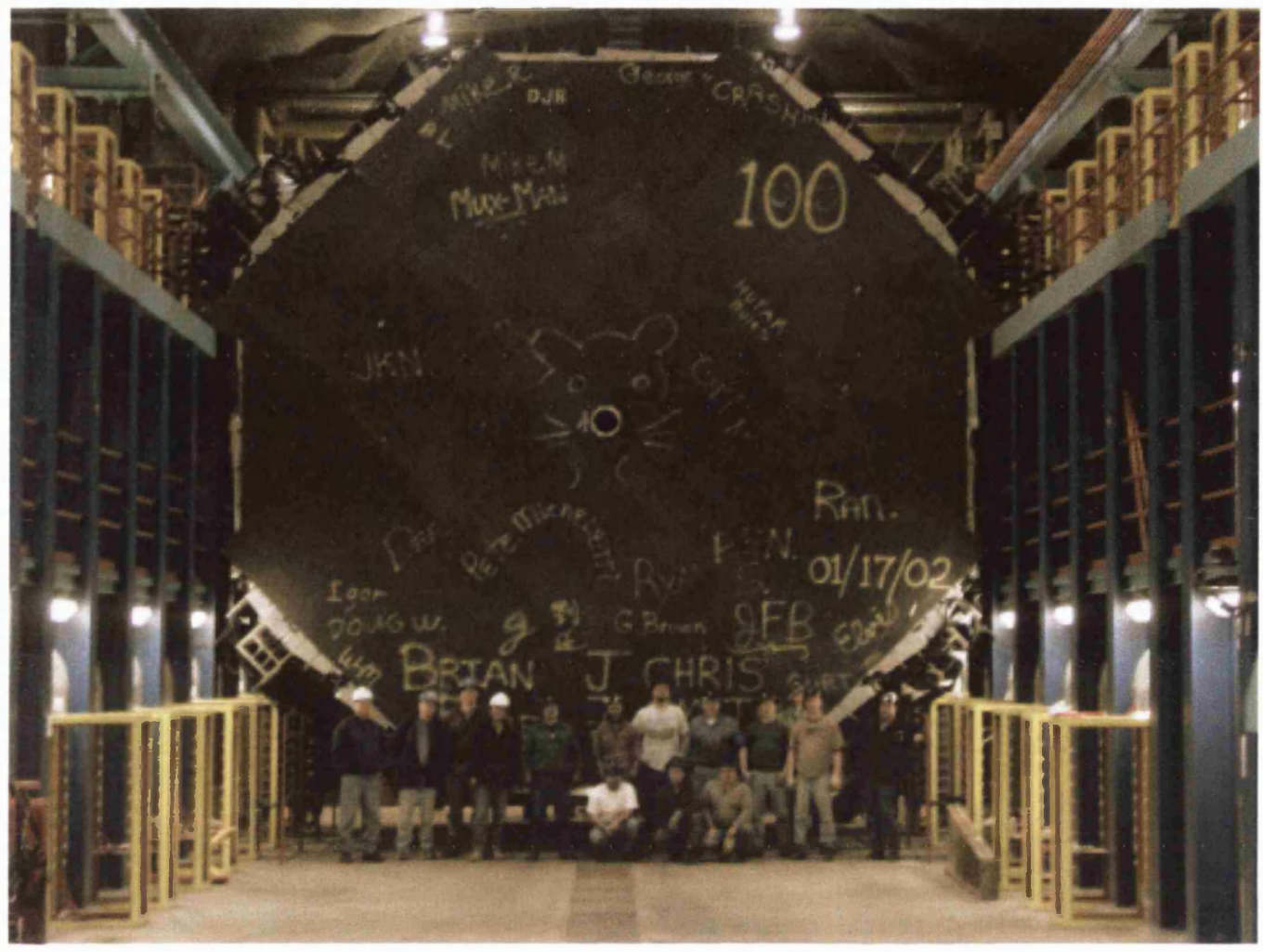

Figure 4.4: The uncompleted Far Detector just after the raising of the 100th plane. The edges of the scintillator modules can be seen behind the steel and the clear fibres, protected and light-tighted within a black conduit, can be seen to run from the detector to yellow crates on the uppermost and lowermost levels. These crates hold the Multiplexing (Mux) boxes which in turn hold the phototubes.

unique pattern of light for a particular event.

The phototube signals are sampled and digitized by the Front End electronics which are modified versions of the Viking chip made by IDE AS of Norway. The chip contains 32 channels of shaping amplifiers and the circuitry samples and holds signals before digitizing each in turn. The dynode signals from each tube are monitored by the electronics and are used to trigger digitizations. The Front End electronics are read out into the DAQ via VME crates controlled by single board computers. 
In the cavern, the VME crates are located on the middle level, (see Figure 4.4), along with LeCroy 1440 high voltage supplies for the phototubes and Pulser boxes (used to inject light into the planes for calibration purposes). The crates on the upper and lower levels are used to hold the Multiplexing (MUX) boxes which in turn hold the phototubes.

\subsubsection{The Near Detector}

The Near Detector is located at Fermilab approximately $1 \mathrm{~km}$ downstream of the NuMI target. It weighs 980 tons and is made up from 282 planes each in the shape of an elongated octagon 3.8 metres high and 4.8 metres wide. The Near Detector is fitted with a coil to produce the $1.5 \mathrm{~T}$ magnetic field; however, unlike in the Far Detector, the coil hole is offset from the central axis by $0.5 \mathrm{~m}$.

The design of the Near Detector was chosen to reduce the amount of steel, scintillator and instrumentation required to make the necessary measurements, without compromising the magnetic field strength. The central beam region has a radius of approximately $25 \mathrm{~cm}$ at the Near Detector and so the neutrino beam is directed at a point offset from the centre of the detector by $0.5 \mathrm{~cm}$, (such that there is a $1 \mathrm{~m}$ separation from the coil hole). Figure 4.5 is a sketch of a Near Detector plane.

The detector is made up of four functionally different components, each with the same cross-sectional design. The first component of the Near Detector is called the Veto part and is the most upstream section. Neutrino interactions detected here are not be used in analysis to prevent against possible end effects and to ensure that there is no background from neutron events. This part contains a total of 0.5 metres of steel.

The second component is called the Target part and this is the region 


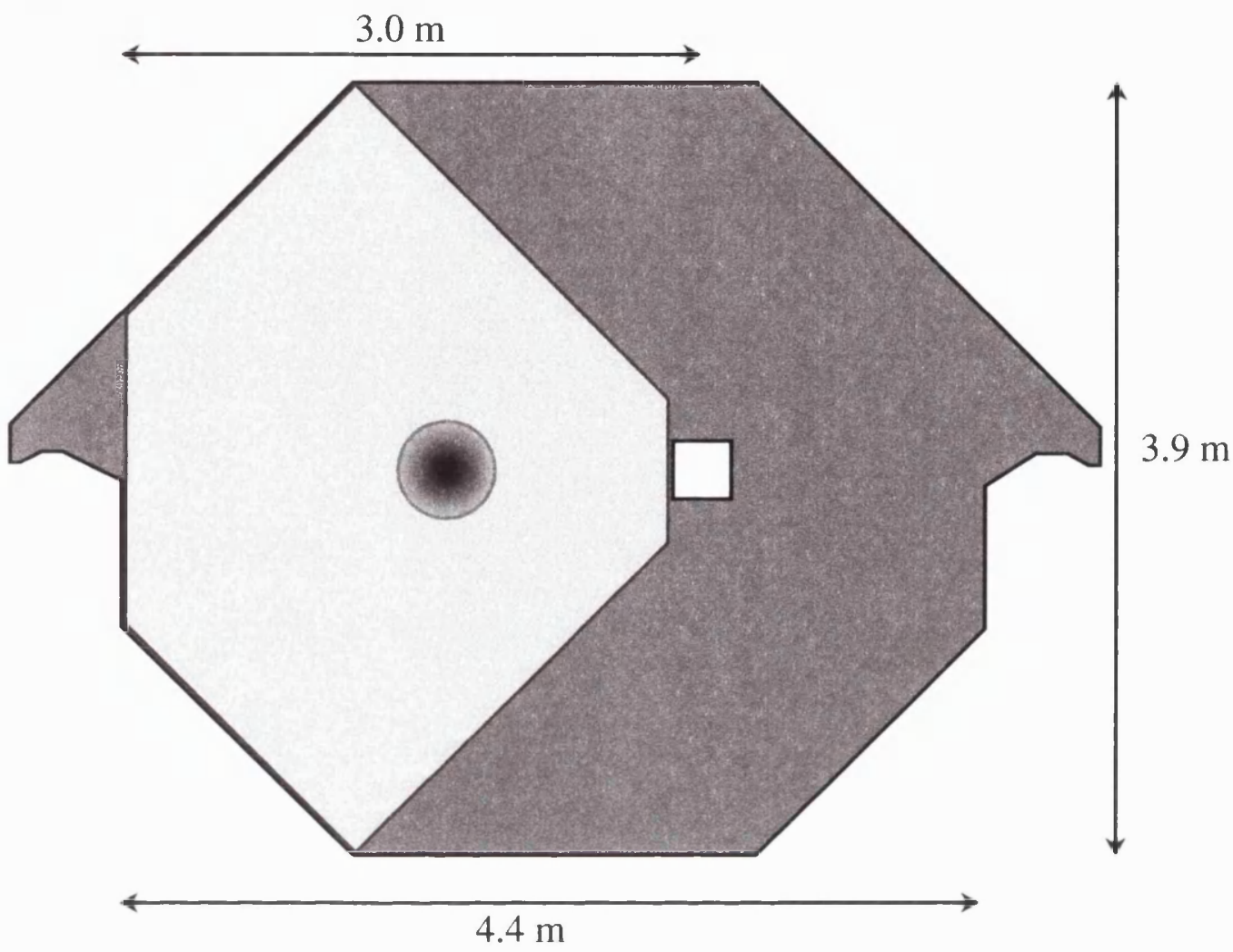

Figure 4.5: A sketch of a Near Detector plane. The coil hole is offset by $0.5 \mathrm{~m}$ from the axis of the detector and the beam is directed $0.5 \mathrm{~m}$ in the opposite direction. The central beam region is $25 \mathrm{~cm}$ as shown by the circular shaded region. Four planes out of five in the three upstream components of the detector are only instrumented in the shaded hexagonal region.

in which interactions will be used for comparison with the Far Detector. It contains 1.0 metre of steel. The third component is the Hadron Shower part and, at 1.5 metres thickness of steel, it is long enough to contain the full showers of all neutrino interactions occurring in the Target part.

The final component is the Muon Spectrometer which is used to either range out muons or to ensure that a measurement of their momentum can be obtained from their curvature in the magnetic field. This part contains 
4.0 metres of steel, which is sufficient to obtain momenta measurements of comparable accuracy to match the Far Detector. Figure 4.6 is a schematic diagram of the Near Detector, showing the instrumented regions.

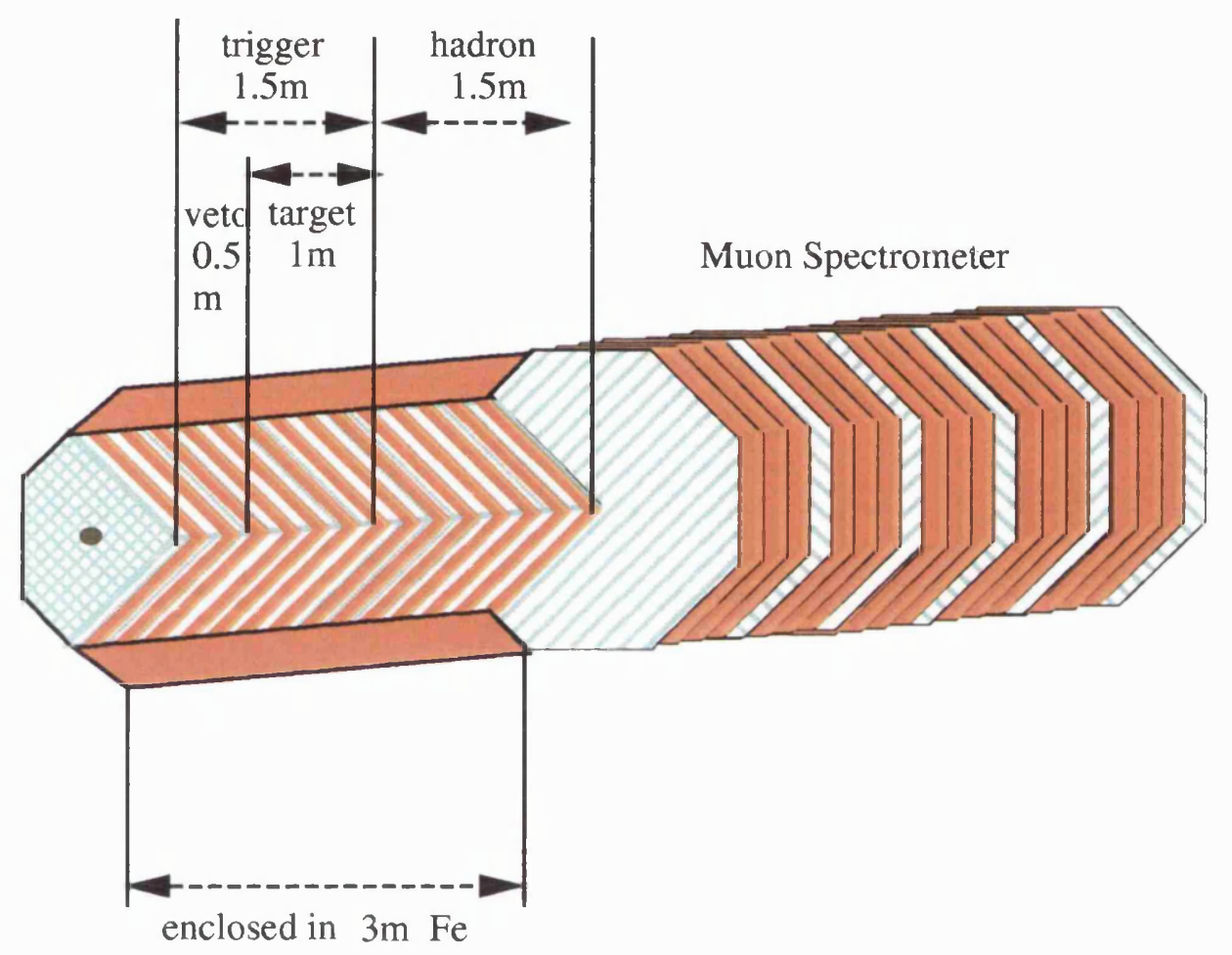

Figure 4.6: The instrumented regions of the Near Detector.

Only the central region of the beam, (within a radius of $25 \mathrm{~cm}$ ), will be used for the Near/Far comparison. Also, the transverse spread of hadronic showers in the MINOS detectors at the energies used is about half a metre. Therefore, it is not necessary to fully instrument each plane. In the three upstream components of the detector, 4 out of 5 planes are only partially instrumented to about $1 \mathrm{~m}$ out from the beam axis. Every fifth plane is then fully instrumented to indicate tracks and interactions outside of the central 
region.

In the Muon Spectrometer section, 4 out of 5 planes are un-instrumented and every fifth is fully instrumented to give total plane coverage. This can be done because the accuracy of momentum measurements from curvature are determined by Coulomb scattering in the steel. Instrumenting the other planes does not improve the measurement.

The Near Detector scintillator strips are read out only on one side. The higher event rates and smaller number of channels (compared to the Far Detector) mean that multiplexing is not used and instead each strip is read out separately. The photodetectors used at the Near Detector are Hamamatsu M64 multi-pixel phototubes where each tube has 64 pixels.

The Near Detector Front End electronics are designed for higher event rates and use high-speed QIE electronics. The QIE system is a deadtime-less, multi-ranging ADC system which divides charge into 19 ns time buckets and digitizes each separately. The digitizations are then reconstructed offline to form hits analogous to those in the Far Detector.

\subsection{Physics Capabilities}

MINOS will measure and compare the rates and energy spectra for both neutral current (NC) and charged current (CC) interactions in both detectors. From the CC energy spectrum, any mode of oscillation can be detected by the depletion of $\nu_{\mu}$ events and a comparison of the Near and Far energy spectra provides information about $\sin ^{2} 2 \theta$ and $\Delta m^{2}$.

In the most simplistic view, a neutrino event in a MINOS detector can be classified as either Long or Short. Long events are mainly $\nu_{\mu} \mathrm{CC}$ events with a background from $\nu_{\tau} \mathrm{CC}$ interactions, (above a threshold of approximately 
$3.5 \mathrm{GeV}$, a tau produced from a $\nu_{\tau} \mathrm{CC}$ interaction can produce a muon and two neutrinos with a branching ratio of $\sim 17 \%$ ). Short events include NC interactions for all neutrino flavours and CC interactions for $\nu_{e}$ and most $\nu_{\tau}$. A sketch illustrating this is shown in Figure 4.7.
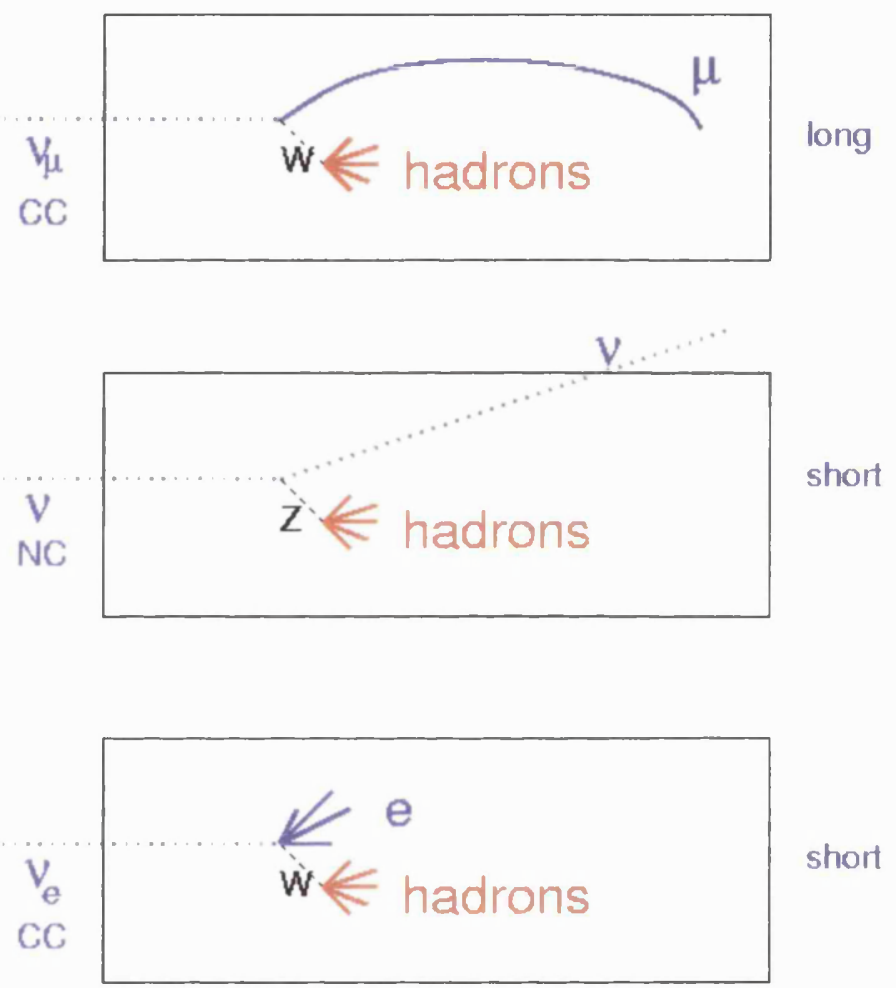

Figure 4.7: Sketches of event topologies in the MINOS detectors. $\nu_{\tau}$ CC events can either look like $\nu_{\mu} \mathrm{CC}$ events, if the tau decays to a muon, or like $\nu_{e} \mathrm{CC}$ events otherwise.

The ratio of ratios $(\text { Short/Long })_{\text {Near }} /(\text { Short/Long })_{\text {Far }}$ as a function of energy, also referred to as the T-test, is a useful measurement for two reasons: firstly, it eliminates most of the problems due to differing rates at the Near and Far Detectors, since any beam-dependent systematics cancel to first order in the ratios. Secondly, it is sensitive to oscillations not only because of the falling number of Long events with $\nu_{\mu}$ depletion but also because most of 
the $\nu_{\tau}$ and all of the $\nu_{e}$ will be Short showering events. The sensitivity of this measurement to $\nu_{\mu} \rightarrow \nu_{\tau}$ oscillations is shown in Figure 4.8 after 2 years running. For maximal mixing the T-test can measure $\Delta m^{2}$ down to $\sim 10^{-3} \mathrm{eV}^{2}$.

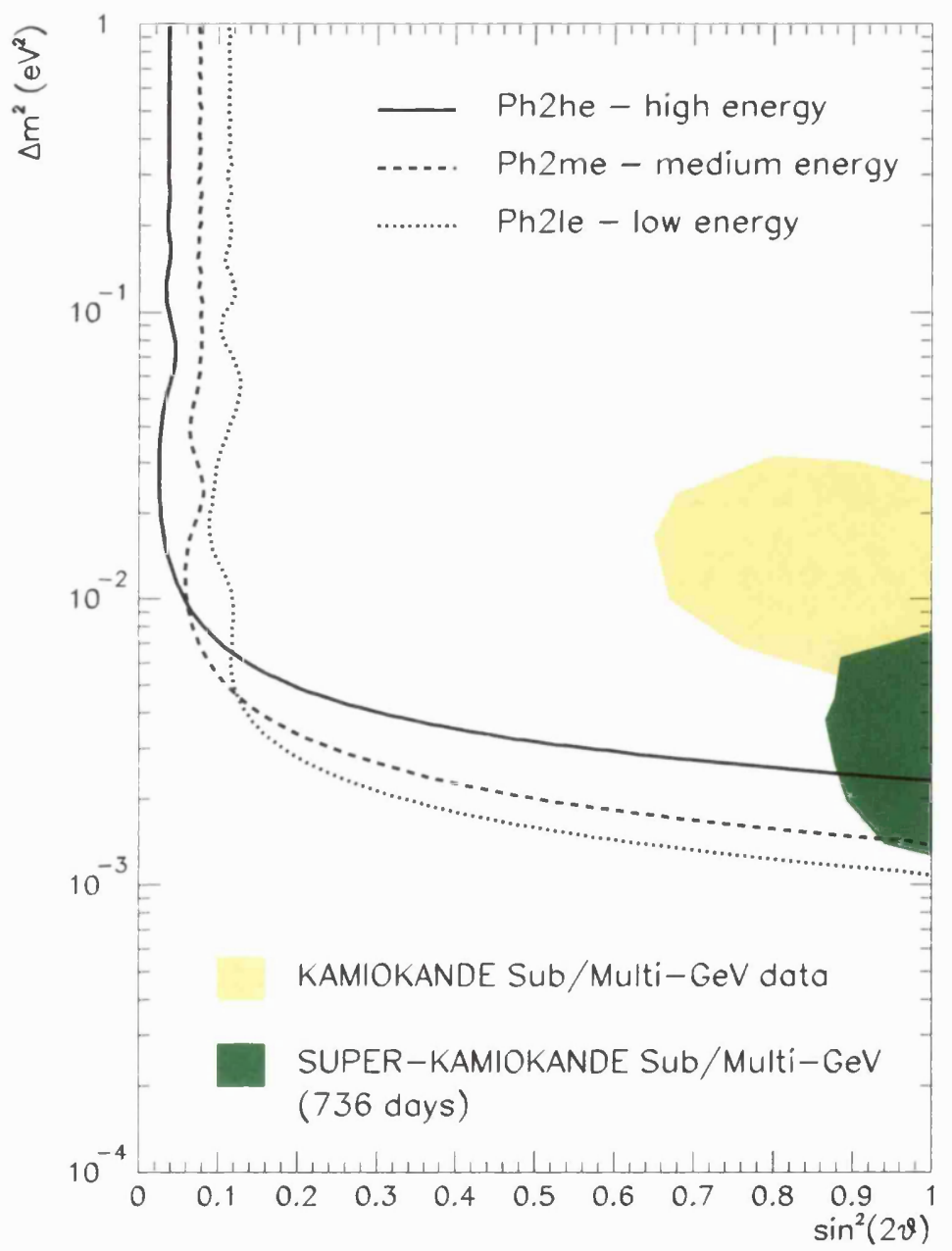

Figure 4.8: Sensitivity of MINOS to $\nu_{\mu} \rightarrow \nu_{\tau}$ oscillations from the T-test measurement. The $90 \%$ exclusion limit for each of the beam configurations is shown assuming no oscillations and after 2 years running. Regions of parameter space favoured by Kamiokande and Super-Kamiokande are also shown.

The T-test is dominated by the statistical error due to the lower number 
of Short events than Long events. The cross-section for NC events rises sharply at small visible energy, therefore an accurate calibration will improve the measurement by allowing the low energy cut-off to be reduced, thereby increasing the NC statistics.

The most sensitive technique for measuring the oscillation parameters available to MINOS is via the $\nu_{\mu} \mathrm{CC}$ energy spectrum (Long event). To compare the Near and Far measurements, the Near spectrum is used in conjunction with a hadronic production Monte Carlo in order to predict the Far Detector spectrum if there were no oscillations. Then, deviations of the measured Far spectrum from this extrapolated Near spectrum indicate that neutrino oscillations have taken place. The oscillation signature appears as a depletion of events at particular energies. The position of the dip then indicates $\Delta m^{2}$ and its magnitude is related to $\sin ^{2} 2 \theta$. Absolute energy calibration is therefore very important for an accurate measurement of $\Delta m^{2}$. The sensitivity of this measurement to $\nu_{\mu} \rightarrow \nu_{x}$ mixing is shown in Figure 4.9 after two years running. For maximal mixing, assuming no oscillations, $\Delta m^{2}$ down to $\sim 8 \times 10^{-4} \mathrm{eV}^{2}$ can be excluded.

MINOS also has some ability to distinguish between hadronic and electromagnetic showers using event topology. Looking for $\nu_{e}$ CC appearance MINOS can modestly improve the limits set by CHOOZ and Super-Kamiokande. Figure 4.10 shows the $90 \%$ exclusion limits after two years running. 


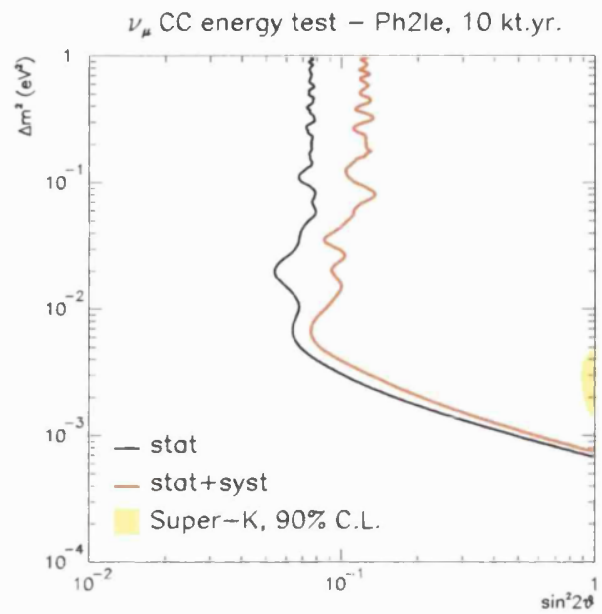

Figure 4.9: $90 \%$ exclusion limits from MINOS for $\nu_{\mu} \rightarrow \nu_{x}$ oscillations from a measurement of the $\nu_{\mu} \mathrm{CC}$ energy spectrum after 2 years running and for the Low energy beam (assuming no oscillations). Beam systematic errors are also included at $2 \%$. The Kamiokande allowed region is shown in yellow.

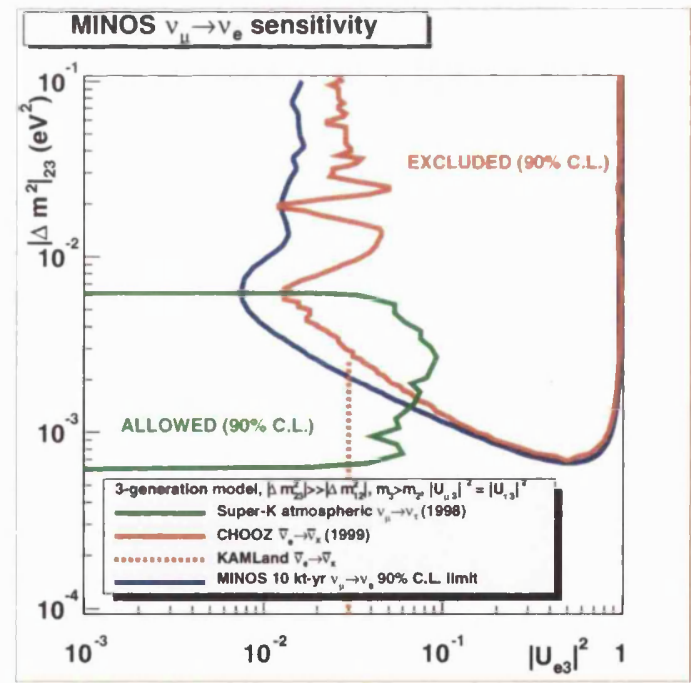

Figure 4.10: $90 \%$ exclusion limits from MINOS for $\nu_{\mu} \rightarrow \nu_{e}$ oscillations from a measurement of the $\nu_{e} \mathrm{CC}$ appearance after 2 years running. MINOS will be able to modestly improve the limits set by CHOOZ and Super-Kamiokande (shown). 


\section{Chapter 5}

\section{Calibration Overview}

\subsection{Importance of Calibration}

A measurement that relies on comparing the responses of two different detectors is clearly very sensitive to systematic errors due to mis-calibrations. Further, the measurements that MINOS intends to make of the neutrino oscillation parameters strongly depends on the absolute energy calibration. Therefore, the question of calibration for MINOS comprises: removing detector variations within each detector, relating energy deposits from different detectors and finally translating detector responses for different types of particles to energy in $\mathrm{GeV}$.

A number of procedures have been developed to tackle the issue of calibrating the MINOS detectors, and a Calibration Detector has been constructed in order to measure the absolute energy scales for different particles. 


\subsection{Relative Calibration}

An important part of calibrating the MINOS detectors is the ability to relate measurements made at one detector to those made in another. The first step towards achieving this is to make the response of each individual detector uniform. That is to say, variations in factors such as phototube gain, connector efficiency and scintillator light output must be corrected for, such that events in different parts of a particular detector can be meaningfully compared.

The next step is to relate energy deposits in one detector with those in another. This can be done by using particles of known energy and comparing the detector responses. MINOS will use stopping muons from cosmic rays. (Stopping muons must be used, rather than all cosmic ray muons, because the cosmic ray energy spectra are different at the Far and Near detectors. The Far detector is deep underground and so the cosmic ray spectrum there is biased towards higher energies.) MINOS is designed to achieve a $2 \%$ relative calibration.

\subsubsection{Charge Injection}

Charge from the photodetectors is sampled by the Front-End electronics, then digitized. The linearity of the ADC scale with charge can be checked and calibrated by injecting a known amount of charge at the Front-End. The injected charge follows the same path as real signals through the electronics and the subsequent digitizations give the relationship between injected charge and ADC counts. This procedure calibrates the ADC readout channels to $1 \%$. 


\subsubsection{Light Injection}

A Light Injection (LI) calibration system [51] has been designed for the MINOS detectors. The system has a number of purposes: to measure and correct for non-linearities in the response of the phototubes with light, to correct for gain drifts over time and to measure the position of the single photoelectron peak for each phototube pixel.

Bright blue LED light is used to illuminate each of the wavelength shifting (WLS) fibres embedded in the scintillator strips. The light is injected into each scintillator module via Light Injection Manifolds (LIMs) which are located near the optical connectors. Each LIM services a number of WLS fibres the shape of which is designed to maximize the amount of light incident on each fibre. The light absorbed by the WLS fibres follows the same readout path as real signals and is digitized by the Front-End electronics.

The LED light is also monitored by PIN diodes. These have been measured to be stable to $0.5 \%$ over a 2 month period and in tests no evidence of non-linearity with light has been observed [52]. Thus, they provide a good measurement of the actual amount of light injected and are suitable for calibrating the response of the detectors to the specified level. Signals measured by the PINs are shaped to look like phototube signals and are then digitized by the Front-End electronics.

The LEDs themselves are housed in Pulser Boxes which provide the means for controlling the pulsing remotely. All aspects of the pulsing, including the frequency and LED light level can be varied. The Pulser Boxes also provide the fibre fanout which transports light to each of the LIMs for each of the scintillator modules. A schematic diagram of the LI system is shown in Figure 5.1.

Each LED is monitored by two PIN diodes; one low gain and one high 


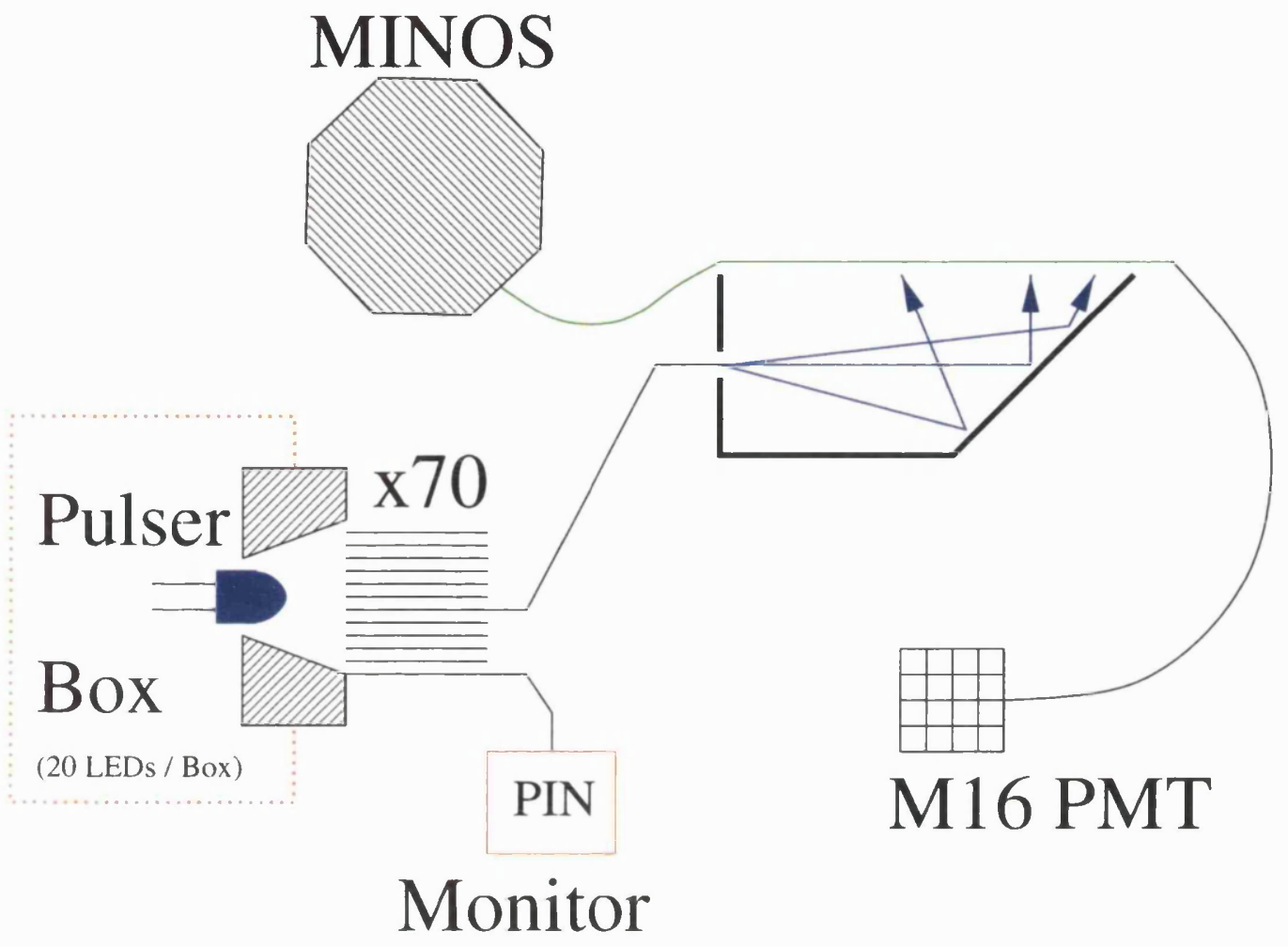

Figure 5.1: A schematic diagram of the Light Injection system.

gain. Having this arrangement ensures that there is always a PIN response regardless of the amount of light being injected (for example, if a high light level saturates the high gain PIN diode, the low gain PIN diode is still able to make a measurement). Having two PIN diodes also ensures that there is always some redundancy should one fail.

The LI system has two usual data taking modes. The first is a dedicated LI mode in which the LEDs are pulsed at a range of different light levels sufficient to monitor the response of the detector over the entire dynamic range of the ADC readout. Thus, a comparison of the response of the PIN with the response of the phototubes yields information about non-linearities in the response of the phototubes with light. These gain curves can then be used to correct real signals offline. 
The second LI data taking mode involves pulsing each LED at a single light level during normal data taking. This allows the gain curves, measured using the longer, dedicated runs described above, to be scaled to account for gain drifts within runs.

As MINOS detectors become operational, the longer runs needed to map out the gain curves are taken approximately once a month and the gain drift is monitored every 20 minutes, running concurrently with normal data taking.

\subsubsection{Strip-to-Strip Calibration}

Strip-to-strip calibration is the process of accounting for variations in the response of each readout channel upstream of the photodetectors. This calibration accounts for differences in scintillator light output, WLS fibre light collection efficiency, cable and connector transmission efficiency and phototube gain and quantum efficiency. Cosmic ray muons at each detector are used for understanding these differences.

A cosmic ray muon track can take a variety of paths through a detector. Each track must therefore be corrected on a hit by hit basis to account for differences in channel response due to track angle (affects the path length through the scintillator) and hit position along the strip (affects the amount of signal attenuation in the optical fibres before reaching the photodetectors).

The number of $\mathrm{ADC}$ counts corresponding to the passage of a muon (after applying the charge injection and light injection calibration and tracking corrections), are used to characterize the response of each readout channel. The result of correcting for these differences is that, individually, the response of each detector is now uniform for all readout channels.

There is one caveat of this technique which arises from the trigger require- 
ment that for an event to be written to disk, a minimum number of planes must be hit within a particular time period. A result of this is that at the edge of a detector, the angular acceptance of cosmic ray muon events will be different to the acceptance in the centre. Due to the energy dependence of the cosmic ray flux with angle, this in turn can result in a difference in the average muon energy at the edge to that in the centre of the detector. In terms of physics measurements however, this problem can be overcome by only considering events contained within a certain fiducial volume of the detector.

\subsubsection{Stopping Muons}

The final stage for relative calibration is to use cosmic ray muons that stop in the detector. Since energy loss and range for muons is well understood, the particles can be tracked and the response at a particular point along the track corresponding to a particular muon energy can be measured. This measurement allows all energy deposits to be expressed in terms of Muon Energy Units (MEUs), and so events in different regions of different detectors become directly comparable.

\subsection{Absolute Calibration}

In order to successfully calibrate the absolute energy scales in the detectors, it is essential to understand the response of different types of particles in a MINOS style calorimeter. To achieve this, the MINOS Calibration Detector (CalDet) has been constructed at CERN to take data in a number of test-beams from the Proton Synchrotron (PS).

The CalDet has the same basic design as the two larger MINOS detec- 
tors, and so its measurements can be directly applied to the Near and Far detectors. The CalDet will measure muons, electrons and hadrons of known energies from the test-beams and will characterize the response of each. The results will ultimately relate the energy in $\mathrm{GeV}$ of each particle type to MEUs. The CalDet as been designed to achieve this to $5 \%$ accuracy.

Calibration work carried out at the CalDet, specifically on the topic of strip-to-strip calibration, is the subject of Chapter 6 . 


\section{Chapter 6}

\section{The Calibration Detector}

Accurate calibration demands that differences in muon, electron and hadronic energy deposition in the MINOS detectors are well understood. To accomplish this a smaller version of the Near and Far Detectors, the Calibration Detector (CalDet) is being exposed to particles of known energies produced in test-beams at CERN.

A further purpose of the CalDet is to refine the relative calibration procedures. Muons are the cornerstone of the MINOS calibration, however, at the Far detector the rates are so low that a useful sample requires a month of data taking. At the CalDet, there are many muons passing through the detector all the time from several sources making it an ideal place to carry out thorough investigations. This chapter presents an in-depth study of the muons at CalDet with regards to the calibration of MINOS.

\subsection{Description of the CalDet}

The CalDet is intended to be a segment from one of the larger detectors. It is $1 \mathrm{~m}$ by $1 \mathrm{~m}$ in cross-section and is 60 planes in length, with the same pitch 
between planes as for the Near and Far Detectors. It weighs approximately 12 tons, (compared to the $\sim 5$ kton Far Detector) and its size reflects the volume required to contain showering particles of the energies produced in neutrino interactions at the Near and Far Detectors. A photograph of the CalDet is shown in Figure 6.1.

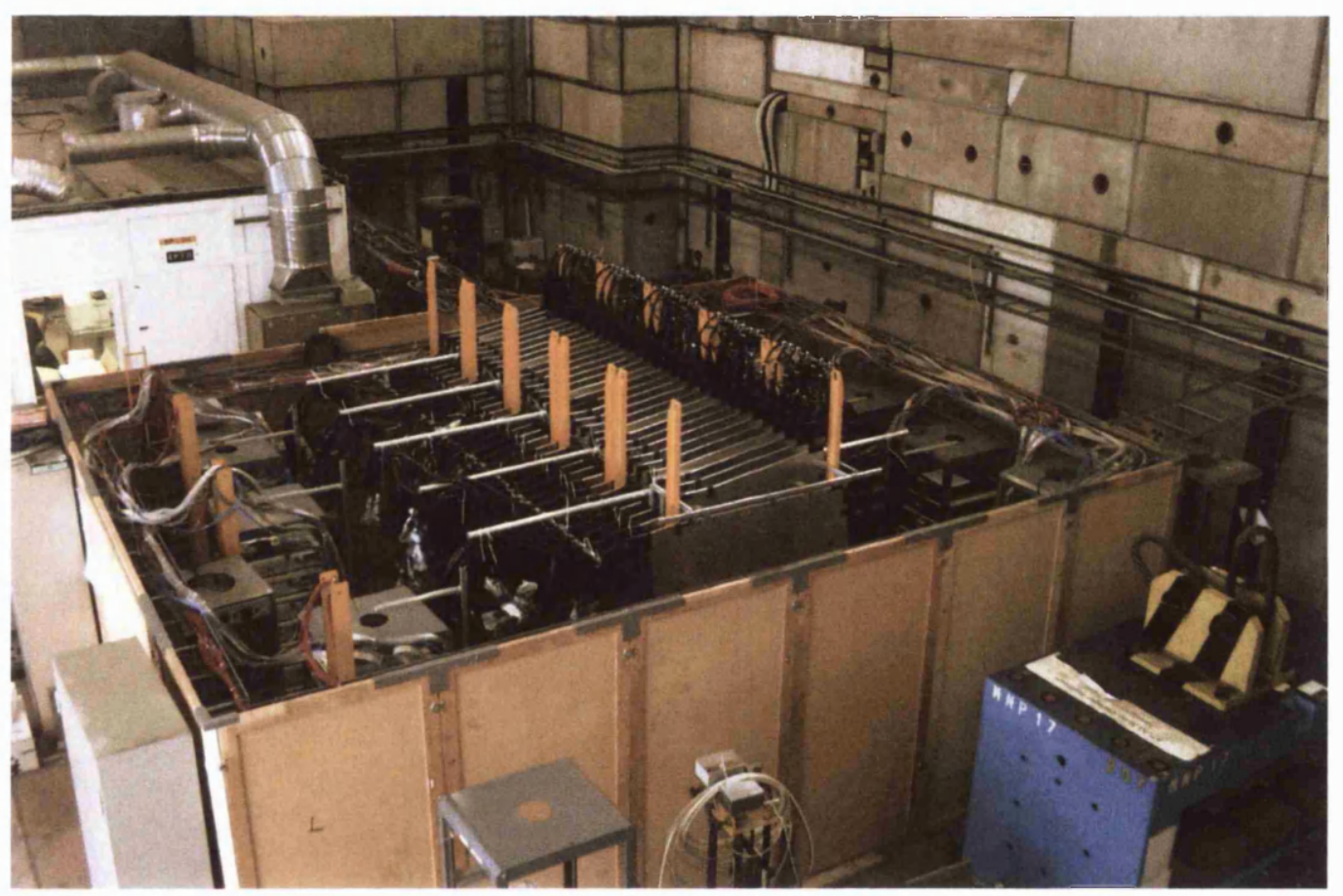

Figure 6.1: A photograph of the CalDet. The fibre optic readout cables can be seen which transport light from the aluminium-covered scintillator planes to the photodetectors. Notice the pattern of the cables corresponding to the alternating vertical and horizontal orientations of the scintillator strips.

A significant difference between the CalDet and the Near and Far Detectors is that there is no magnetic field. Studies of the influence of a magnetic field on the energy deposited in an iron/scintillator calorimeter have shown that neither the mean of the total energy distribution nor the detector resolution are significantly affected by the field [53]. The effect of a magnetic field 
on the phototubes has also been studied [54] [55]. The MINOS phototubes are housed in MUX boxes which provide some magnetic sheilding and the magnetic field strength is expected to be below 5 Gauss at the phototubes. The studies showed that at these field strengths there was no significant change in the gain or the probability of cross-talk, although some reduction in collection efficiency for the edge pixels is expected [56]. For these reasons, and also because of the difficulty associated with engineering a magnetic field comparable to the Near and Far fields, the CalDet was designed not to be magnetized.

Each plane consists of a layer of steel of thickness $2.5 \mathrm{~cm}$ and an active layer of scintillator. The scintillator layer is constituted from $24,1 \mathrm{~m}$ length strips (with the usual $1 \mathrm{~cm}$ by $4.1 \mathrm{~cm}$ cross-section), arranged and fixed in an aluminium covering to form a module. Within a module, the WLS readout fibre for each strip are collected and routed to connectors at the edge of the module.

The CalDet has optical fibre readout from both sides of each scintillator strip. The modules are connected to modified Far Detector MUX boxes each containing 3 Hamamatsu M16 multipixel phototubes, (each phototube has 16 pixels). Each MUX box reads out signals from one side of 2 planes and the CalDet, unlike the Far Detector, is not multiplexed. The CalDet is read out on one side of each plane via a $6 \mathrm{~m}$ length of clear optical fibre (between the scintillator module and MUX box), and via a 4m length of green WLS fibre on the other side. This was done in order to simulate typical signal attenuation due to green fibre expected at the Far Detector, where the scintillator strips can be up to $8 \mathrm{~m}$ long.

After photomultiplication, signals from the phototubes are sampled and 
digitized by the Front-End electronics ${ }^{1}$. A threshold value on the dynode signal from each tube is set such that only dynode signals above one-third of a photoelectron trigger digitization. The electronics is also designed to carry out pedestal subtraction and sparsification, (the process of removing digitizations below a certain threshold on the grounds that they cannot be distinguished from pedestal).

Surviving digitizations are passed into the DAQ stream where a simple dedicated trigger processor looks for certain hit patterns in order to decide whether or not to keep the data. Once a trigger condition has been satisfied, a window of $\sim 200 \mathrm{~ns}$ is opened and all hits from that period are written to disk. These triggered time windows are nicknamed snarls rather than events since each snarl can potentially contain more than one physics event. However, at CalDet, the beam conditions are set such that each snarl typically only contains one physics event. This is also true when taking cosmic ray muon data.

A caveat of using the Far detector electronics in the test-beam is that they are designed for very low event rates and consequently introduce a component specific deadtime of $5-10 \mu s$ after each readout. This means that a chip receiving a dynode trigger will read-out and subsequently be dead asynchronously with the other chips for $5-10 \mu s$. The times for which each chip is dead are logged such that partially detected events can be identified offline and are not included in analyses.

\footnotetext{
${ }^{1}$ For the work presented in this thesis, Far Detector Front-End electronics were used. However, CalDet will also take data using Near Detector electronics in order to compare the responses.
} 


\subsubsection{The Test Beam}

The CalDet has been exposed to the T11 and T7 test-beams at the CERN PS. The T11 beam has a range of particle energies between 0.5 and $3.5 \mathrm{GeV}$ and the $\mathrm{T} 7$ beam has a range between 0.5 and $10 \mathrm{GeV}$. The beams consist of pions, protons and electrons, and muons from pion decay. The particles are directed to the beam area during a number (between 1 and 3 ) $\sim 0.5$ second spills within a $\sim 15$ second cycle.

Cerenkov detectors are available that are set-up to detect electrons above a particular energy. Also, a Time of Flight (TOF) system has been developed in order to distinguish pion and proton events in the detector. The relevant trigger conditions that are applied at the CalDet are: a hit in the LI Trigger PMT (which is illuminated at a high light level every time any LI LED is flashed), a TOF coincidence or a collection of hits for which, in a group of 5 consecutive planes at least 4 of the planes have a hit. There are also special modes, such as a pedestal mode, where the electronics self trigger.

Cosmic ray muons events are also obtained during the beam exposure, between spills. Outside of test-beam runs, the CalDet is housed in an enclosure in the same hall as the beam areas. During this time, cosmic ray data can also be obtained. In both the beam area and the enclosure, during the summer months, there is a further source of high energy muons from the PS itself.

\subsection{Calibration Tools}

The first step of the calibration procedure for MINOS involves correcting the response of the detector for differences in scintillator light output, WLS fibre collection efficiency, optical transmission efficiency and phototube gain 
and quantum efficiency. A number of analysis tools were developed to make the measurements shown in this chapter. The most widely used are described in the following sections.

\subsubsection{Event Display}

A simple event display has been developed which was used at the beginning of the CalDet running predominantly to help find and fix problems in the readout chain. It also proved useful later to help develop an efficient track finder. An example of the display is shown in Figure 6.2. The four views of the detector are displayed schematically for a muon event, (two sided readout of alternating vertical and horizontal strips).

\subsubsection{Low Light Level LI Spectra}

At low light levels, the number of photoelectrons emitted from the photocathode follows a Poisson distribution. This original distribution is smeared out by the multiplication in the phototube, producing a much wider ADC spectrum. A function which fits the spectra produced from pulsing the Light Injection system at low light levels has been developed [57]. The function is the convolution of a discrete Poisson with a Gaussian distribution and its form is given below: 

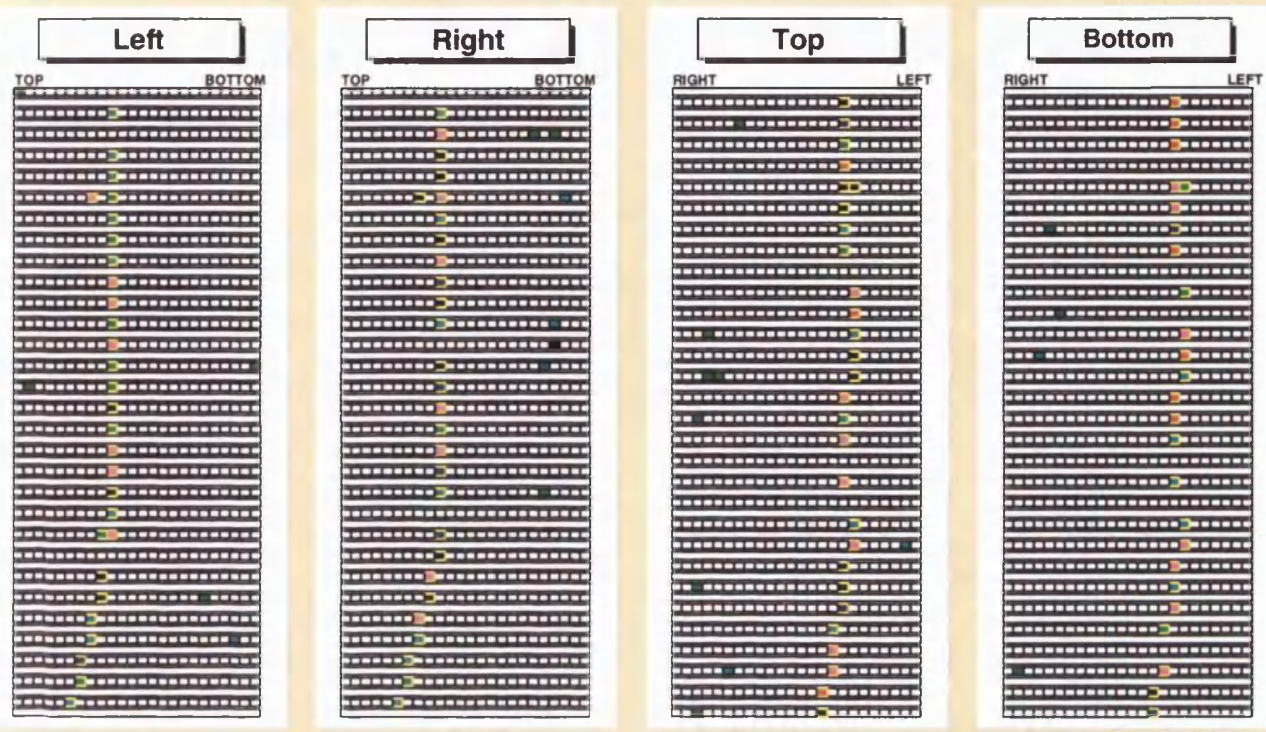

Key:

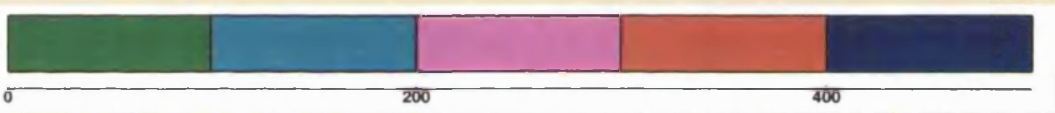

Figure 6.2: An example of the event display developed for the CalDet. The front of the detector is at the top of the display, therefore this event is likely to be a beam related muon. The Top and Bottom views represent the channels associated with the vertical scintillator strips and the Left and Right views the horizontal strips, (Left and Right are with respect to the detector)

$$
\begin{aligned}
f(x)= & N e^{-\lambda} \frac{1}{\sqrt{2 \pi} \sigma_{p e d}} e^{-\frac{\left(x-x_{\text {ped }}\right)^{2}}{2 \sigma_{\text {ped }}^{2}}}+ \\
& N\left(1-d_{f}\right) \sum_{n=1}^{n=12} \frac{e^{-\lambda} \lambda^{n}}{n !} \frac{e^{-\frac{\left(x-x_{\text {ped }}-n x p e\right)^{2}}{2\left(\sigma_{p e d}^{2}+n \sigma_{p e}^{2}\right)}}}{\sqrt{2 \pi\left(\sigma_{p e d}^{2}+n \sigma_{p e}^{2}\right)}}+ \\
& N d_{f}\left(1-e^{-\lambda}\right) \frac{e^{-\frac{\left(x-x_{p e d}-x_{p e} / d_{s}\right)^{2}}{2\left(\sigma_{p e d}^{2}+\sigma_{p e}^{2} / d_{s}\right)}}}{\sqrt{2 \pi\left(\sigma_{p e d}^{2}+\sigma_{p e}^{2} / d_{s}\right)}}
\end{aligned}
$$


where $N$ is the number of entries in the histogram to be fitted; $\sigma_{\text {ped }}$ and $x_{\text {ped }}$ are the width and mean of the pedestal in the spectrum; $\sigma_{p e}$ and $x_{p e}$ are the width and mean of the one photoelectron peak; $\lambda$ is the mean number of photoelectrons produced at the photocathode, as expected from Poisson considerations.

One of the features of these spectra is a small peak between the pedestal and single photoelectron peak. It can be understood by considering photons that pass through the photocathode and hit the first dynode. The dynodes are made of a similar material to the photocathode and so the photon can produce a photoelectron at the first dynode, resulting in a smaller signal at the anode. The parameters related to this effect in the function are: $d_{f}$, related to the probability that a photon will pass through the photocathode and strike the dynode to produce a photoelectron and $d_{s}$, related to the amount by which the photomultiplication is reduced in such cases. An example of the fit, can be seen in Figure 6.3.

This function is used to fit low light level spectra for each channel. The position of the single photoelectron peak from the fit (the gain), is returned and can be used in conjunction with the LI drift points to correct all the hits in the detector into photoelectrons.

\subsubsection{Track Finder}

A track finder has been developed to select muon related hits from beam events in each of the four views separately. Only event topology is used to select track candidates, no cuts on the ADC value associated with a hit are applied. The tracking algorithm searches for coincident hits in consecutive planes by attempting to follow the path of the track forwards through the detector. Upon finding a hit, the tracker looks in the next plane of the same 


\section{Fit to Low Light Level Spectrum}

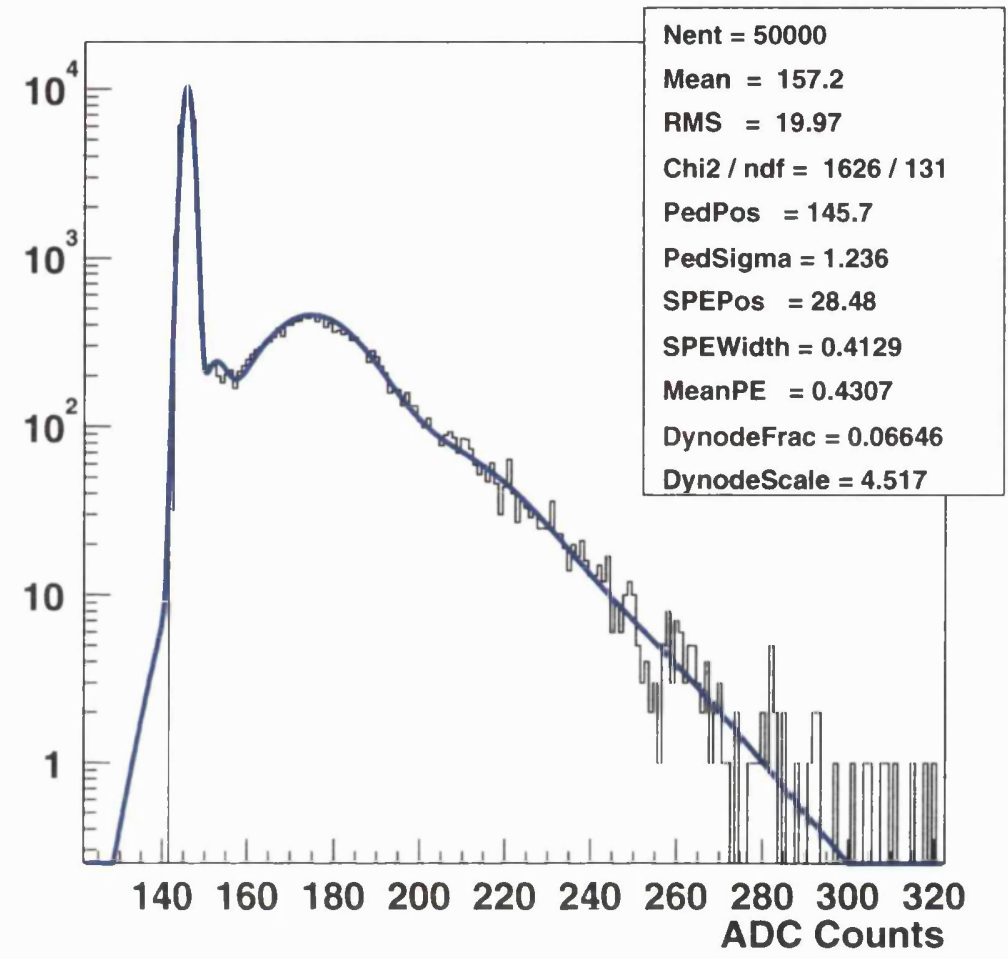

Figure 6.3: An example of a fit to a constant, low light level spectrum. The position and widths of the pedestal and single photoelectron peak are parameters of the function, as well as the mean number of photoelectrons and two parameters used to model the effect of photons that pass through the photocathode and strike the first dynode.

orientation for a hit in a user specified strip range. A sketch of an example tracking pattern is shown in Figure 6.4.

The searching continues until the track either stops or leaves the detector or a hit pattern is found that sets a veto flag. In the latter case, the event fails and is not used in the analysis. The veto is required to remove showering events from the muon sample. The following cuts are chosen to remove electrons and showering pions from the muon sample during test-beam data 


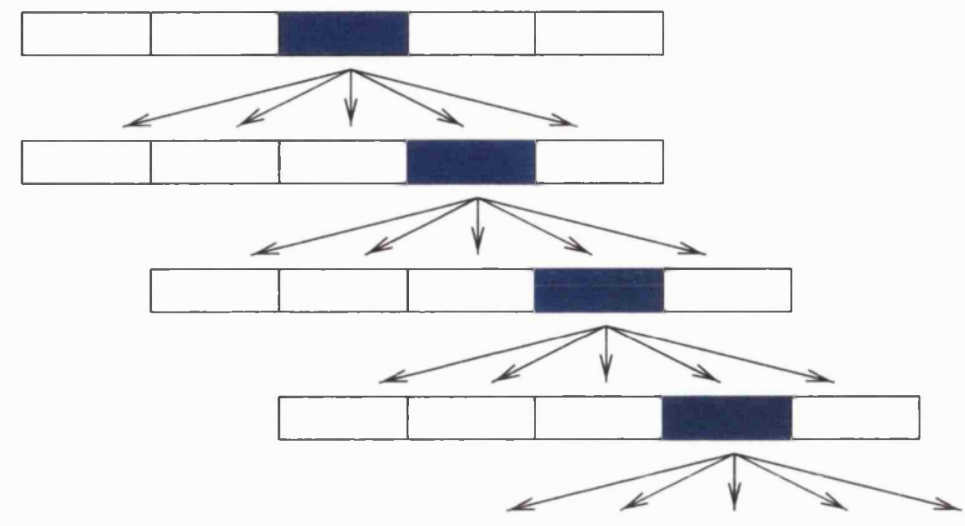

Figure 6.4: An example of the searching pattern used to find track related hits in the CalDet.

taking:

- No plane has three hit strips along the path of the track

- No two consecutive planes (of the same orientation) each have two or more hit strips along the track

If a track appears to stop in the detector, the tracker will continue to look for track hits for two planes (of the same orientation) and then will assume that the track has ended. The tracker will then start looking for a new track in the remainder of the detector. In this way, should there be large gaps along the track for any reason, the tracker is still able to find all the hits.

Looking again at Figure 6.2, the strips surrounded by a yellow border can now be identified as those that were selected by this tracking algorithm. Once the track finder has attempted to find hits in all four views, events with a track spanning at least 4 planes in each view are accepted as muons. 


\subsubsection{Track Finder Performance}

\section{Event Selection Efficiency}

To investigate the ability of the track finder to select muon events and reject showering events, samples of 5000 muons and 5000 pions at a range of energies were generated in a GEANT simulation of the CalDet. Typical light levels and attenuation effects measured at the CalDet are used simulate hits in terms of ADC counts, (to produce typical CalDet Poisson efficiencies for each channel). The samples of particles are generated with a Gaussian spread of $1.5 \%$ in energy. Cross-talk is not included in these simulations.

The events have been passed through the track finder algorithm and Figure 6.5 shows the fraction of events accepted as a function of particle energy for the muon and pion samples. For the muon sample, the selection efficiency is $91 \%$ at $1 \mathrm{GeV}$ and quickly rises to $98-99 \%$ at higher energies. (The lower efficiency at lower energy arises from a loose angle cut which is applied to the real data and so has been applied here for consistency. Demanding that the average track angles in an event are less than $10^{\circ}$ with respect to the beam direction in both orientations is a quick way to reject cosmic muons from the sample. At the lower energies, there is a larger chance that multiple scattering will cause the event to be rejected by the angle cut.)

Most of the pions are removed by the shower cuts, however at lower energies, up to $25 \%$ topologically do not appear to shower. A closer look shows that many of these tracks are shorter than muon tracks of the same energy. This indicates that they are not depositing energy like a minimum ionizing particle and so could have consequences for the calibration if included.

Figure 6.6 shows the last hit plane for muons and pions at $2 \mathrm{GeV}$. Demanding that the pions also stop within the muon peak removes most of the 
Number of Selected Events as a function of Energy

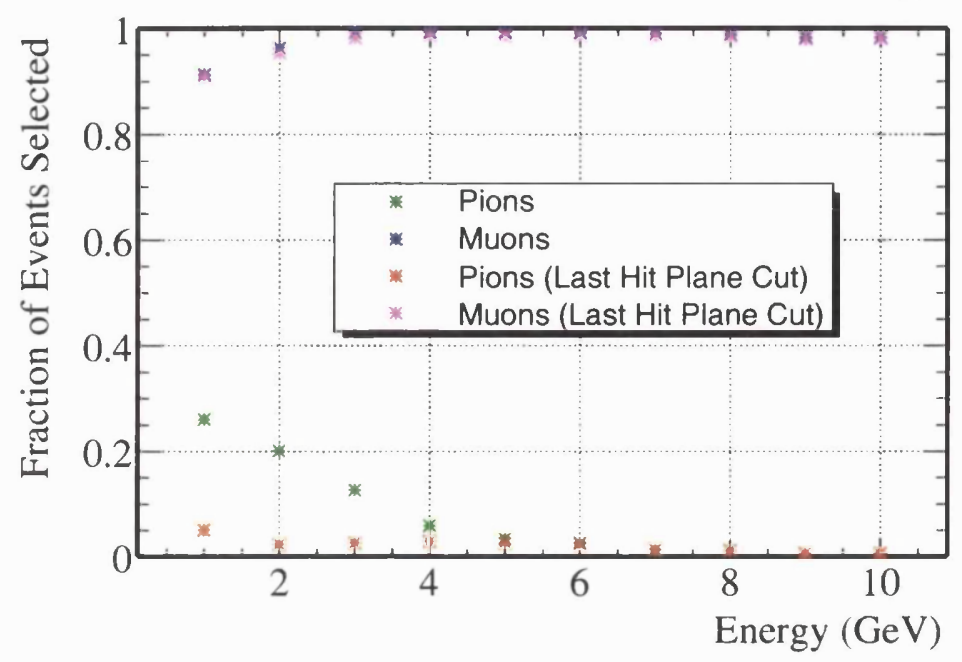

Figure 6.5: Fraction of muon and pion events selected using the track finder algorithm for a range of energies in a Monte Carlo simulation of the CalDet. A cut on the last hit plane in the event removes the majority of the pion events accepted by the track finder.

selected pions as shown in Figure 6.5. Those pions remaining are essentially indistinguishable from muons in terms of topology on an event by event basis, although, from the Bethe-Bloch relation, small differences of order $2-3 \%$ in the average $\mathrm{dE} / \mathrm{dx}$ in iron are expected.

The selection statistics for a typical 1 hour CalDet run in the T11 testbeam at a beam energy of $2 \mathrm{GeV}$ are shown in Table 6.1. These, along with a plot of the stopping plane for the selected events within $\pm 10^{\circ}$ of the beamline, shown in Figure 6.7 can be used to estimate the approximate contents of the beam sample at this energy.

The peak of the stopping plane distribution corresponding to the muons is at $\sim 56$ ( 56 inches of steel traversed). Based on a Gaussian fit to this peak, the fraction of muons and pions that behave like minimum ionizing particles 


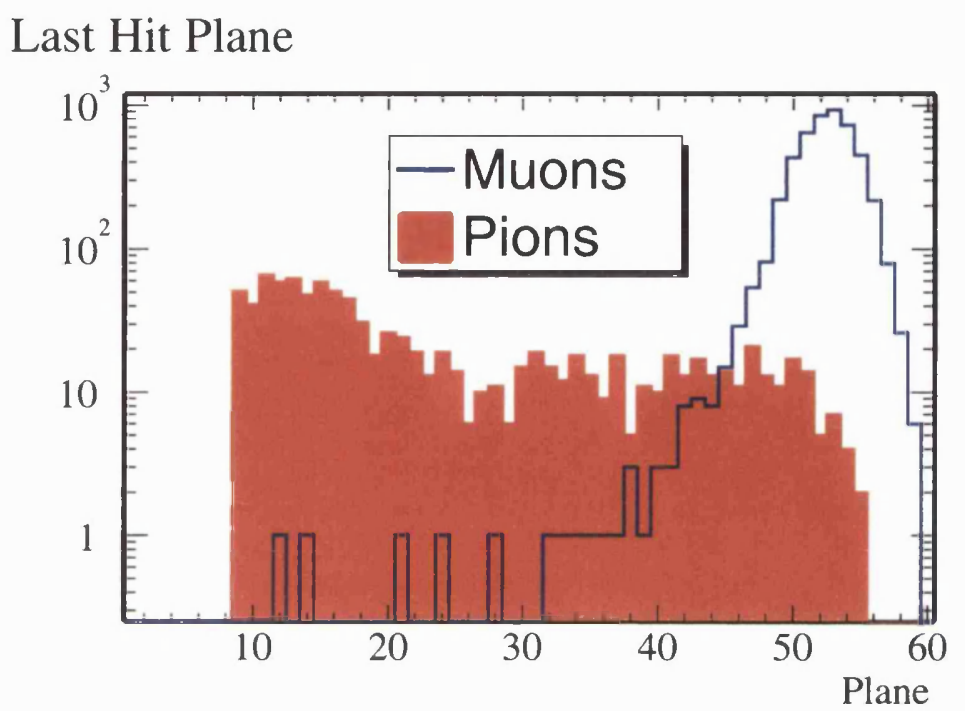

Figure 6.6: Last hit plane for $2 \mathrm{GeV}$ muon and pion events selected by the track finder algorithm in a Monte Carlo simulation of the CalDet. The pions that stop earlier in the detector than the muons are not minimum ionizing and so are removed from the sample using a cut on this quantity.

(MIPs) in the successfully tracked sample is $\sim 16 \%$ and pions (and protons) therefore constitute the remaining $\sim 84 \%$ of the tracks. From Figure 6.5 the number of accepted pions at $2 \mathrm{GeV}$ is expected to be $\sim 20 \%$ with $\sim 1 \%$ being in the peak of the stopping plane distribution. Based on these figures, the fraction of pions in the tracked sample is $\sim 88 \%$ with $\sim 4 \%$ being in the peak of the stopping plane. The fraction of muons in the tracked sample is therefore $\sim 12 \%$.

Using these figures and the knowledge that the tracked sample makes up $7.5 \%$ of the entire triggered sample, the fraction of pions in the data is estimated to be $\sim 33 \%$ and muons from pion decay make up $\sim 1 \%$. Now, using the information from Table 6.1 that $76.5 \%$ of events are flagged as showering events, the fraction of showering events in the data not due to pions can be 


\begin{tabular}{|c||c|c||}
\hline \multicolumn{3}{|c||}{ Run 40171, $2 \mathrm{GeV}$} \\
\hline \hline Cut & \# selected & $\%$ selected \\
\hline \hline All Triggered Beam Events & 148000 & 100 \\
\hline Tracks in Vertical Strips & 38931 & 26.3 \\
\hline Tracks in Horizontal Strips & 36018 & 24.3 \\
\hline Passing Shower Cut & 34716 & 23.5 \\
\hline Tracks in Both Orientations & 20322 & 13.7 \\
\hline Tracks Within $\pm 10^{\circ}$ of Beamline & 11054 & 7.5 \\
\hline
\end{tabular}

Table 6.1: Selection statistics from a CalDet T11 Beam run at $2 \mathrm{GeV}$.

estimated to be $\sim 50 \%$. These events are identified as electron events.

Of the remaining $16 \%$ of the data, $6 \%$ are the tracked events which were not within $\pm 10^{\circ}$ of the beam-line and $10 \%$ are events that were neither flagged as showering event nor were identified as being tracks. The former are higher angle muon and pion events from the beam and the latter are likely to be very short events from the beam which do not shower significantly and are not long enough to be tracked. These events are likely to be pion or proton events. The beam sample is therefore estimated to be constituted by $\sim 48 \%$ pions/protons, $\sim 50 \%$ electrons and $\sim 2 \%$ muons from pion decay.

In summary, the sample initially contains $\sim 2 \%$ muons. After tracking the accepted sample contains $\sim 12 \%$ muons with remainder being pions. After applying a cut on the stopping plane, the surviving sample is $\sim 75 \%$ muons with a $\sim 25 \%$ contamination from pions which behave like MIPs.

\section{Hit Selection Efficiency}

One feature that has not yet been incorporated into the Monte Carlo is the cross-talk between phototube pixels. This leads to phantom hits in the 


\section{Stopping Plane (2 GeV CalDet Run)}

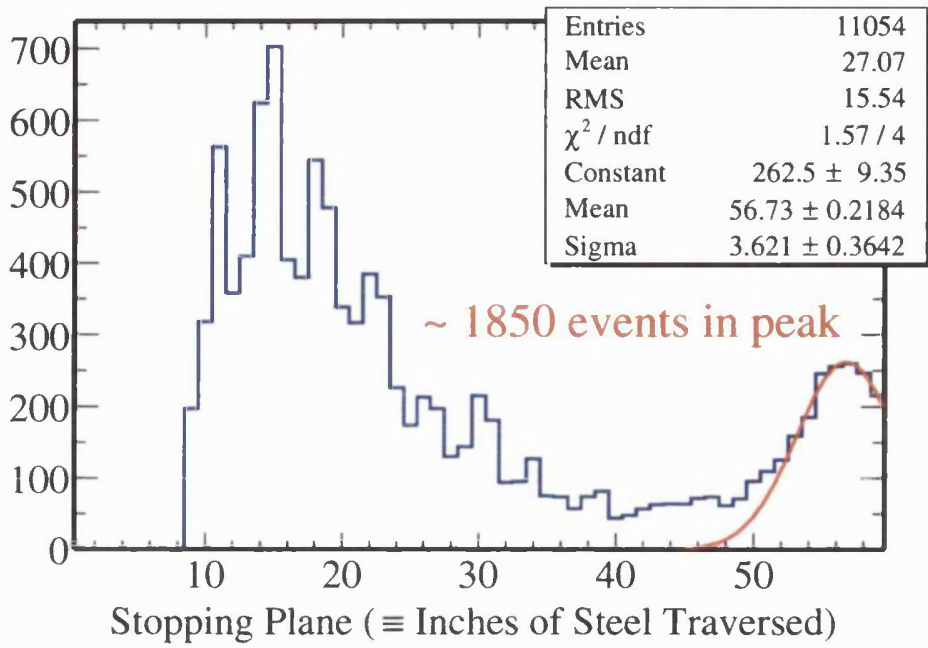

Figure 6.7: Stopping plane for tracked CalDet events in a $2 \mathrm{GeV}$ run in the T11 test-beam. The number of entries in the fitted peak give information about the muon content of the data sample.

detector which will sometimes appear along a track. Cross-talk to the nearest neighbor phototube pixels produces the most significant effect and is at the level of a few percent [58]. The average cross-talk light level for muons is a fraction of a photoelectron and so sparsification usually removes these hits. However, surviving cross-talk hits that are included in the calibration will cause the typical muon response to be underestimated.

The mapping of the strip-ends to the phototube pixels is different on each side of the detector. Cross-talk hits on both sides of the detector from a single strip will therefore not coincide to mimic a real detector hit. For this reason, a simple way of removing cross-talk hits is to take only double-ended hits, that is, hits for which there are corresponding hits on the opposite side of the same strip, for use in calibration. Cross-talk hits therefore cannot be accepted, as, even if hits on both sides of a particular strip produced cross- 
talk hits, the different mapping to the phototube pixels prevents them from a double-ended hit. Using this technique the hit selection efficiencies have been studied for the track finder.

A sample of through-going muons from a $3.4 \mathrm{GeV}$ beam run has been selected. An important quantity to look at is the fraction of hit planes along the track. Figure 6.8 shows a histogram of the fraction of planes hit in each event for the sample, where a hit plane is one which has at least one strip with a hit from both ends. The mean of the distribution is at $\sim 0.8$ indicating that typically 48 out of 60 planes have double-ended strip hits.

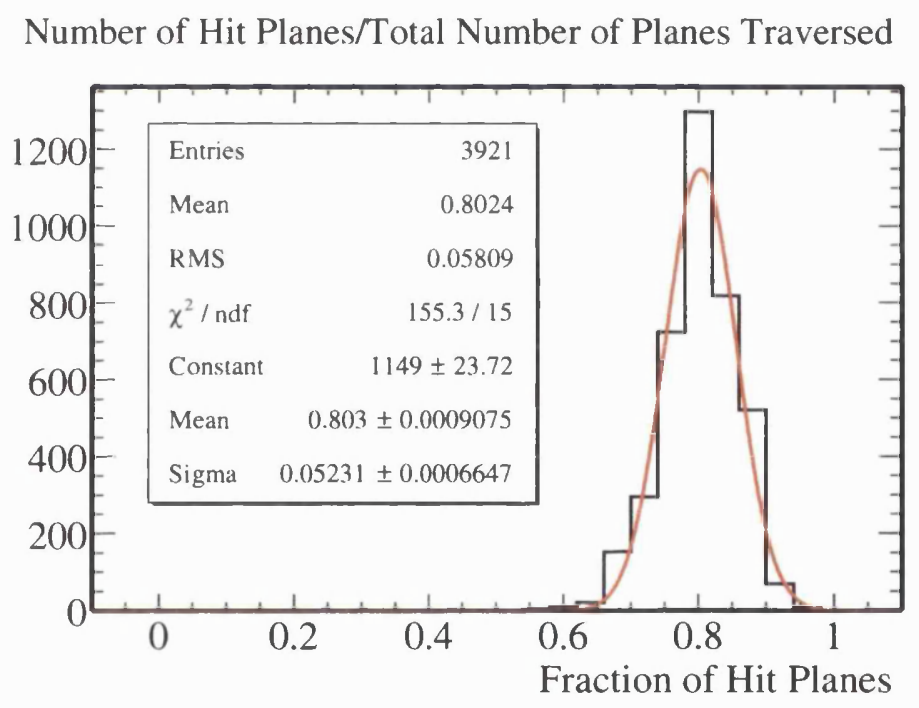

Figure 6.8: The fraction of planes hit for through-going muon events in the CalDet. A hit plane is one in which at least one strip has hits at both ends. It can be seen that typically 48 out of 60 planes contain double-ended hits in an event.

This inefficiency is shown as a function of plane in Figure 6.9. Overlaid is the expected inefficiency based on the light levels observed in the detector (calculated using Poisson probabilities). It can be seen that there is some correlation between the measured and expected inefficiencies, and generally 
the measured values are at the expected level.

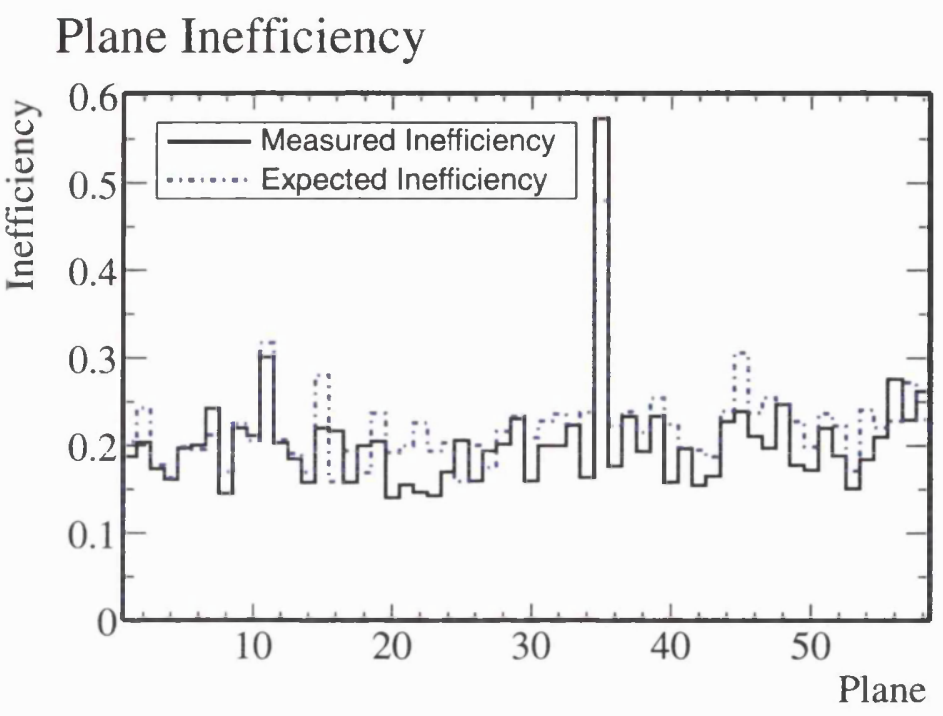

Figure 6.9: Tracking inefficiencies as a function of plane for through-going muons. Both the measured and expected inefficiencies, based on the observed light levels, are shown. The measured values are at the level expected.

To investigate the selection rate of cross-talk hits by the track finder, a useful quantity is the fraction of hits which form a double-ended strip hit compared to the total number of tracked hits. This is shown in Figure 6.10. From Figure 6.8 it was shown that typically 48 out of 60 planes have doubleended hits. Therefore, the average expected number of hits in the detector for such events (assuming that all plane inefficiencies are due to only one side not having a hit) is $48 * 2+12=108$ and the expected fraction of double-ended hits is $(48 * 2) / 108 \approx 0.89$. The mean from Figure 6.10 is at $\sim 0.84$, indicating that $5 \%$ of the hits selected in an event are unlikely to be directly related to the passage of the particle but rather due to cross-talk. This translates into $\sim 6$ selected cross-talk hits per through-going event, a similar occurrence rate as for real single-ended hits. Therefore, using only 
double-ended hits for calibration is a necessary procedure with this tracking algorithm and has been used throughout this analysis.

Number of Double-Ended Hits/Total Number of Tracked Hits

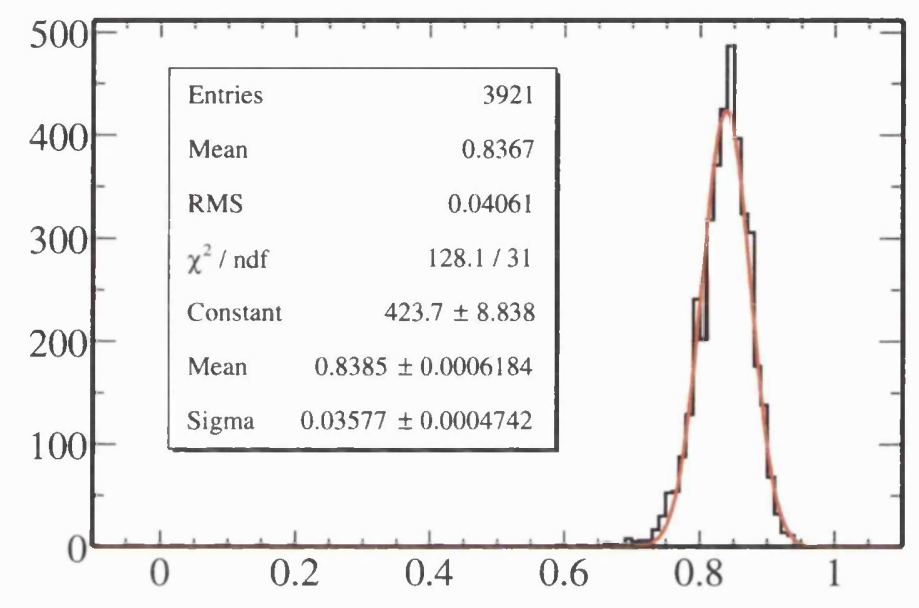

Figure 6.10: Fraction of double-ended hits compared to total number of tracked hits for through-going muons. 


\subsubsection{A Simple Description of Muon Energy Spectra}

The Landau distribution describes the energy loss for a particle passing through thin strips of material. It is therefore assumed that the parameters of this distribution can be used to characterize each of the muon energy spectra. The distribution is characterized by having a narrow peak and a long tail. However, the muon energy spectra themselves have much broader peaks resulting from the effects of photomultiplication in the phototubes. Thus, a reasonable function to fit is the convolution of a Landau with a Poisson distribution, as shown below:

$$
L \otimes P(n)=\int L(\lambda ; \mu, \sigma) \cdot P(n ; \lambda) d \lambda
$$

where $\mathrm{L}(\mathrm{x} ; \mu, \sigma)$ is the Landau distribution with most probable value (MPV) $\mu$ and width $\sigma$; and $\mathrm{P}(\mathrm{x} ; \lambda)$ is the continuous Poisson distribution with mean $\lambda$.

The Landau is itself expressed in terms of an integral, and so calculating an analytical solution for this is difficult. To use the function for fitting, the following approximation is used:

$$
L \otimes P(n) \approx N \sum_{\lambda=1}^{20} L_{r o o t}(\lambda . s ; \mu, \sigma) . P(n ; \lambda . s)
$$

where $L_{\text {root }}$ is the ROOT [59] adapted CERNLIB [60] routine for a Landau distribution; $\mathrm{s}$ is a scale factor which depends on the range of the histogram and the integral has now been replaced by a summation. The fit requires two parameters: the MPV, $\mu$, and width, $\sigma$, from the Landau distribution. Figure 6.11 shows an example of this function. The convolved function is the 
blue, dotted line and the individual Poisson distributions that it is composed from are shown in red.

\section{Muon Energy Spectrum Fitting Function}

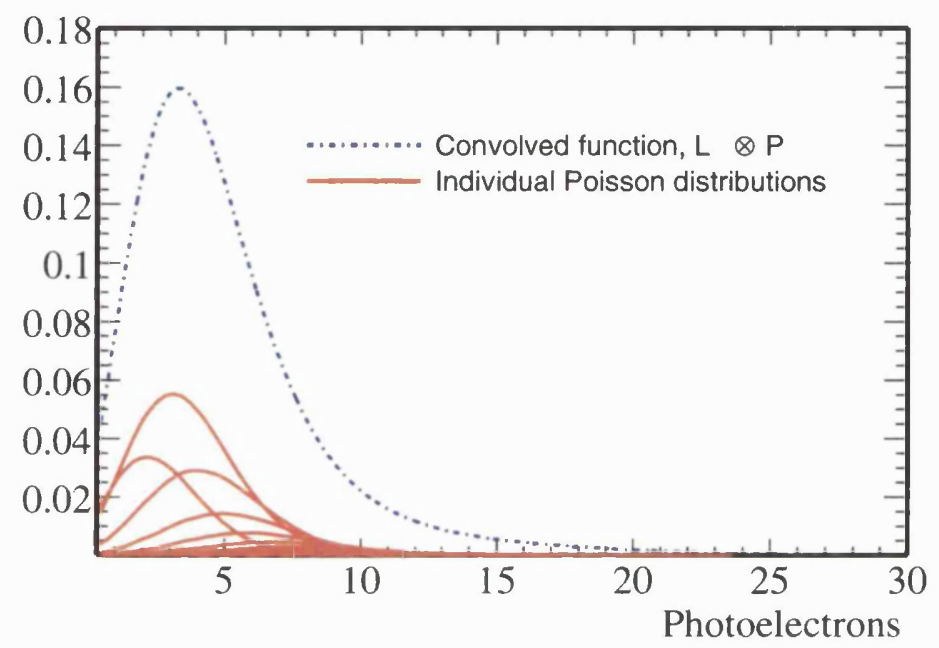

Figure 6.11: An example of the approximate convolution of a Landau with Poisson.

The red lines are the individual Poisson distributions and the blue, dotted line is the convolved function.

An example of the fit is shown in Figure 6.12. It can be seen that the function describes the general shape of the histograms well with an adequate $\chi^{2} / \mathrm{ndf}$.

\subsubsection{Strip End Characterization}

The ADC values for the double ended hits from accepted muon events are histogrammed for each scintillator strip end (each readout channel). The question is then how to summarize each of the histograms to produce a single number corresponding to the response of that channel to the passage of a muon. Four techniques for doing this have been compared:

1. Take the mean of each histogram 


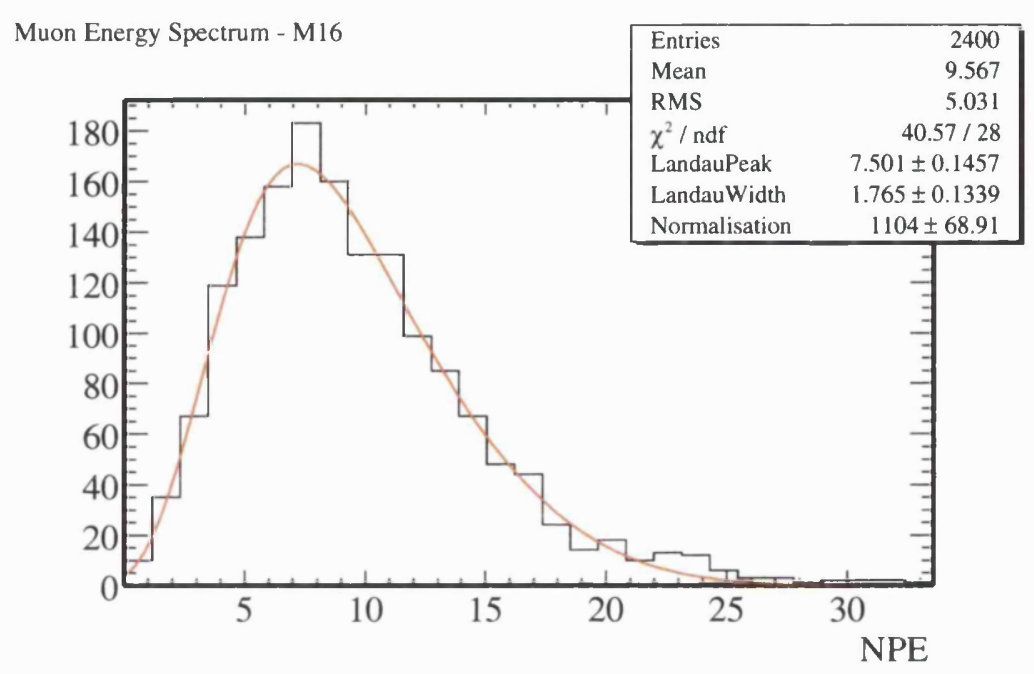

Figure 6.12: An example of a fit to a muon energy spectrum using the simple description outlined in the text.

2. Truncate each histogram to the $90 \%$ level then take the mean

3. Fit each histogram using a simple fitting function, (see Section 6.2.5)

4. Fit each histogram using a more realistic fitting function (see Appendix A)

The last technique was developed using a simulation of the Hamamatsu M16 phototubes [57] which accounts for the photomultiplication at each of the dynode stages. The fitting function is essentially the convolution of a Landau distribution with a Poission distribution to get the initial photoelectron spectrum after the photocathode. The resulting spectrum is then convolved with another Poisson distribution to account for the effects of photomultiplication. The fitting function is described in detail in Appendix A. In both cases where fits are used, the peak of the Landau distribution is used to characterize the histogram. 
The four techniques were then tested on simulated spectra, (created using the phototube simulation described above). Figure 6.13 shows an example result of using each technique on a particular spectrum which peaks at an ADC value equivalent to 4 photoelectrons, (a typical light level observed at the CalDet). The simple fit matches the peak of the distribution reasonably well, but does not reproduce the fine structure of the single photoelectron peak. This results in a $\chi^{2}$ value per degree of freedom greater than 1 . The more complicated function clearly simulates the shape of the spectrum more accurately and has $\chi^{2}$ per degree of freedom close to unity.
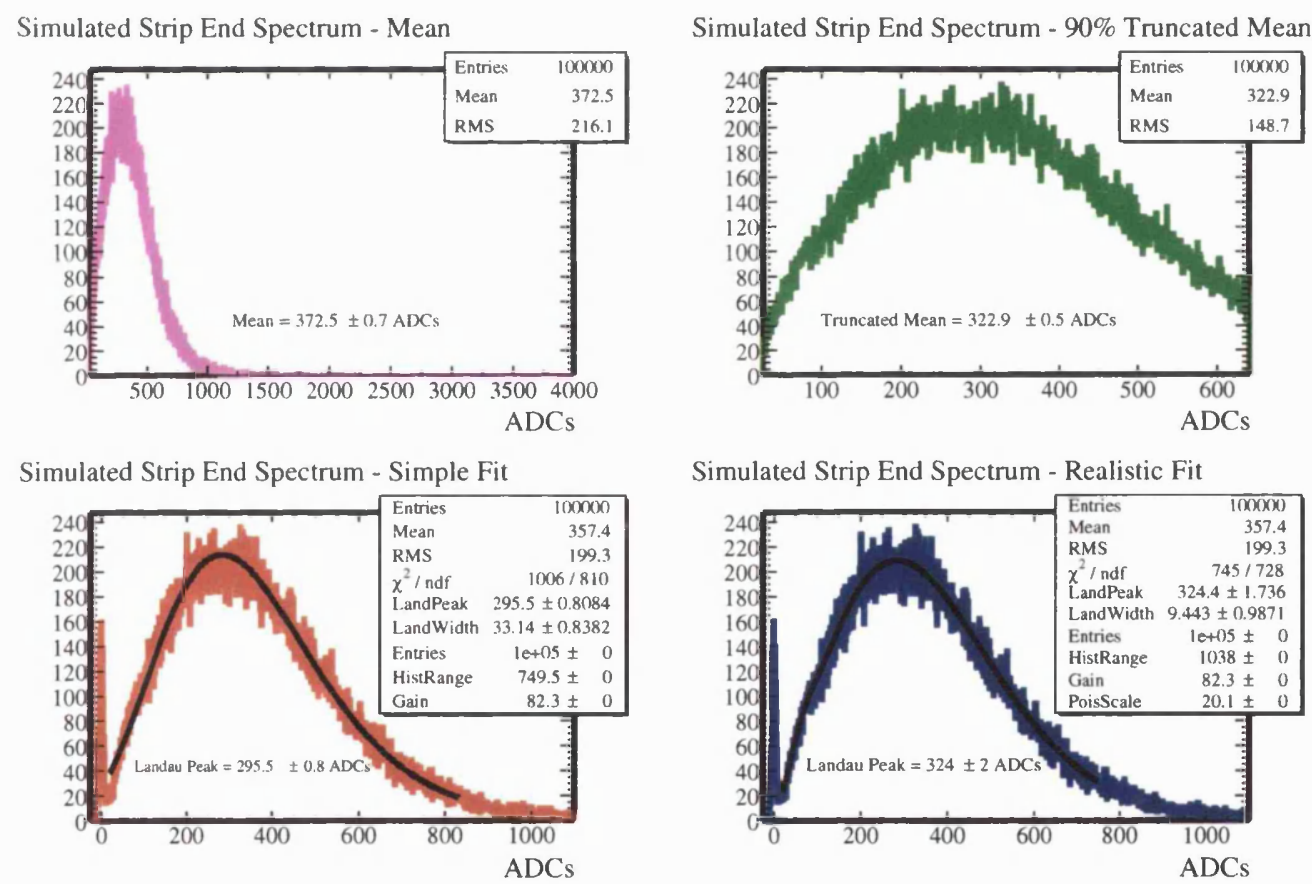

Figure 6.13: The result of characterizing a strip end spectrum using each of the four described techniques. The fits both require parameters for the function that are fixed: the gain, the number of entries and the range over which to fit. The bottom right hand fit also requires a scaling parameter related to the width of the single photoelectron peak. The details of this fit are explained in Appendix A. 
Typically in the Far Detector, there will not be this many entries in each strip-end histogram so it is important to see how the number of entries affects the stability of the means and peaks. 100 spectra were independently generated for each of 10 different numbers of entries in the range 400-10,000. The four techniques were then applied to each of the 1000 spectra. For each technique, a weighted mean is found and the fractional difference from this mean is calculated for each spectrum. Histograms of the fractional differences, (weighted by the fractional error on the difference), for each of the four techniques are plotted in Figure 6.14.
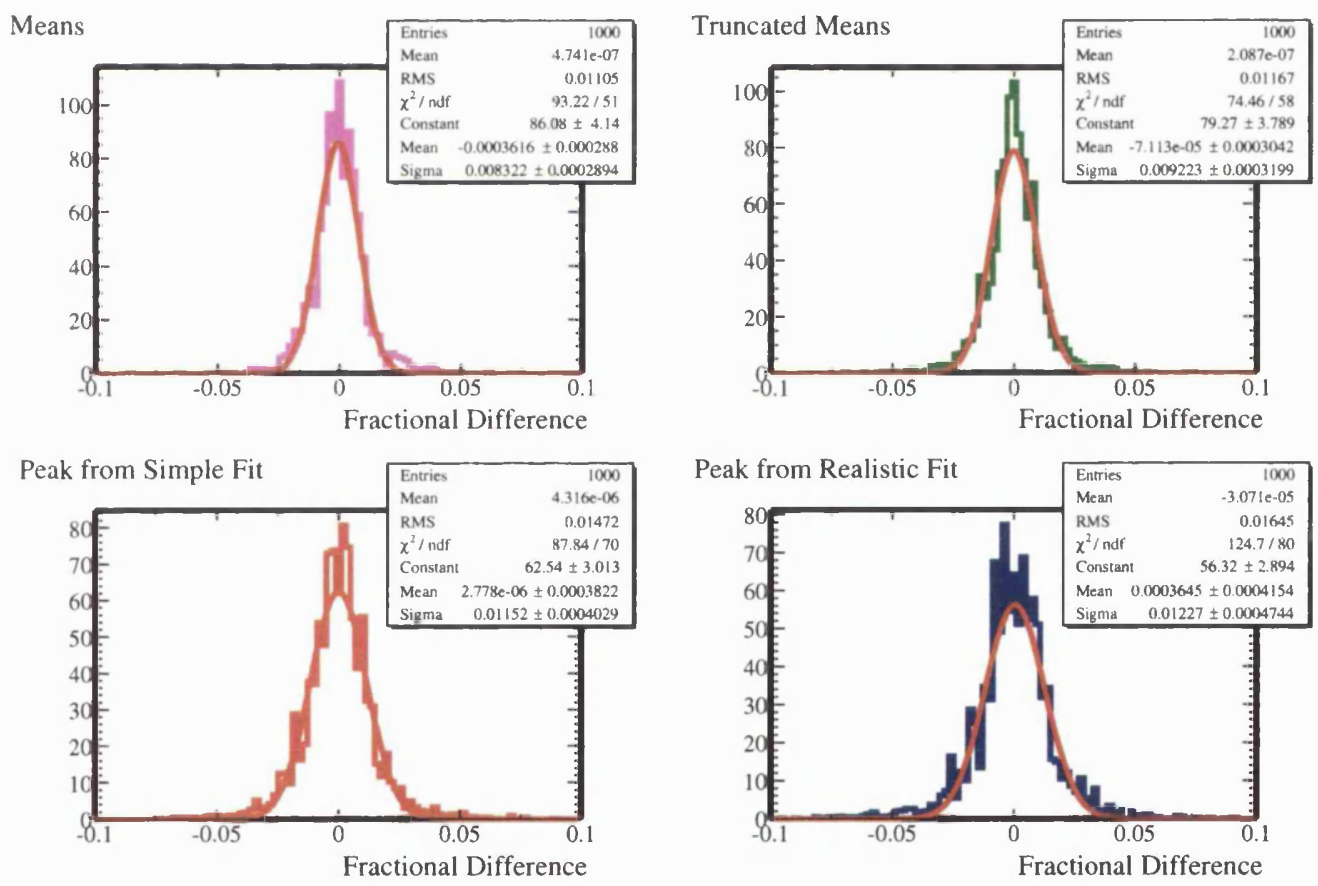

Figure 6.14: The results from obtaining the mean, truncated mean and peaks from two fitting methods when applied to 1000 independently generated spectra of the same light level with different number of entries. Here the fractional differences from the weighted mean are plotted for each of the spectra, for each of the techniques. 
Figure 6.14 implies that the mean would be the best technique having the smallest Gaussian width. However, the mean is expected to introduce systematic errors because of high ADC values in the long tail of the Landau distribution. Plotting the average fractional difference as a function of number of entries for each of the four techniques, as shown in Figure 6.15, it can be seen that the mean does indeed show significant deviations at the $1 \%$ level. The truncated mean too shows some significant deviations from zero, but only at the $0.5 \%$ level. This is also consistent with the long Landau tail being the cause of the deviations.
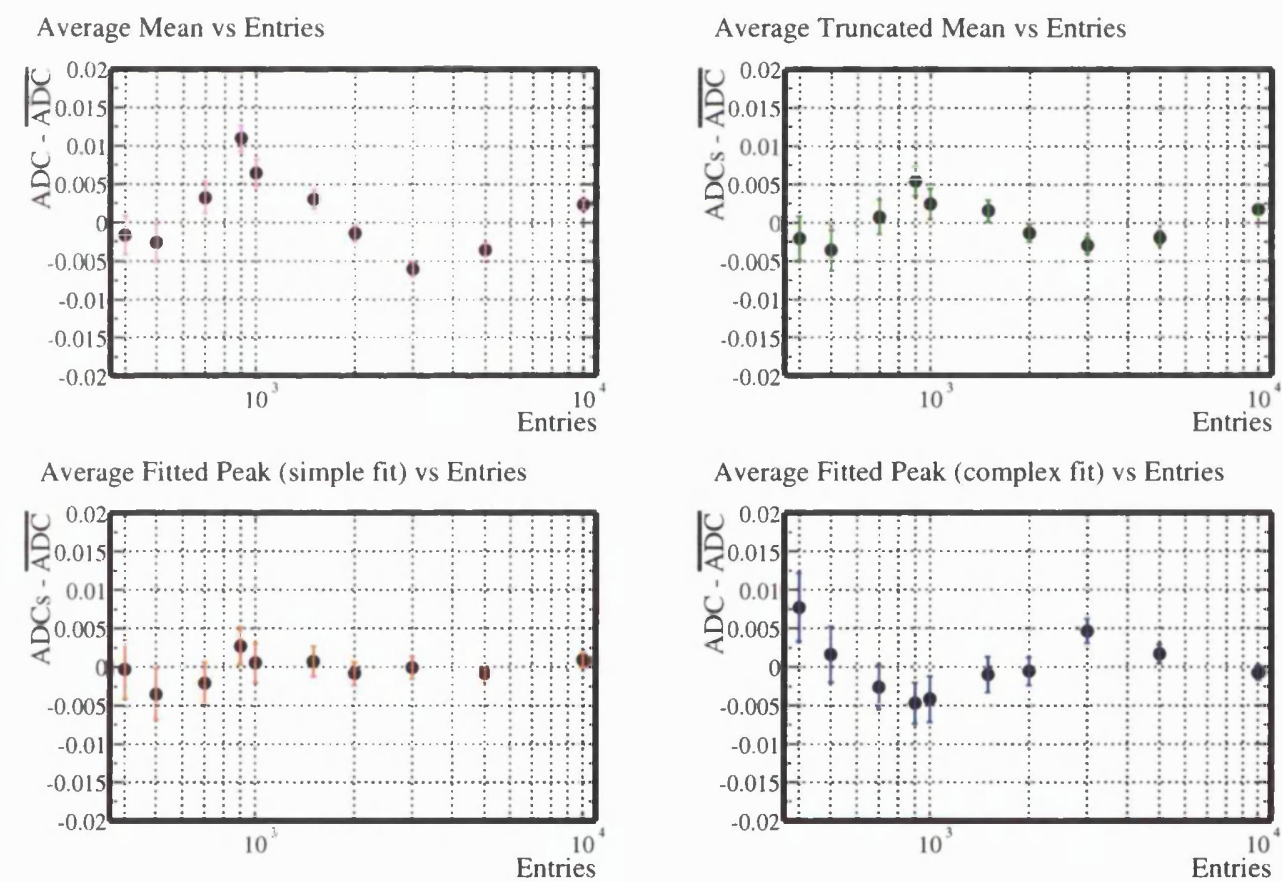

Figure 6.15: The average fractional difference as a function of number of entries for each of the four techniques.

Considering the fits: the simple fit shows the least dependence on number of entries, and therefore the Landau tail effects, (as expected; the fit was developed to account for this effect). Figure 6.14 shows that the Gaussian 
width for the simple fit at $\sim 1.1 \%$ is small enough to be used in strip-tostrip calibration and is therefore a good candidate. The more complicated fit appears also to show significant deviations from zero. This is probably because with low numbers of entries, the more subtle features that the fit is trying to describe cannot be resolved.

Figure 6.14 shows that at four photoelectrons per muon crossing, the $90 \%$ truncated mean exhibits variations of $\sim 0.9 \%$ over the range $400-10,000$ entries. This is acceptable for achieving relative calibration to the $2 \%$ level despite the systematic effects seen at $0.5 \%$, therefore this technique has been adopted over the simple fit because of the overhead involved in performing fits to each strip-end histogram.

\subsection{Calibration Procedures}

Having developed the tools required for the calibration, the procedures used are now outlined. As will be shown later in Section 6.4, at the CalDet, large variations in response are seen across the detector. To achieve the desired $2 \%$ relative calibration, it is therefore necessary to characterize each channel in order to account for these differences.

\subsubsection{Event Selection}

Muon events were selected using the track finding algorithm described in Section 6.2.3. The track finder was originally optimized to track muons and to veto showering events from the beam. However, the algorithm can also be used to track cosmic ray muons. Specific angular cuts are applied to the tracks in order to obtain a sample of muons predominantly from a particular source. Two particular sources are of interest: cosmic rays and the beam. 
The cosmic muons are important for strip-to-strip calibration whereas the beam muons are important for defining a value for the Muon Energy Unit (MEU) for the CalDet (i.e. the response of the detector to a muon of known energy).

In terms of strip-to-strip calibration, to ensure that the calibration constants are consistent throughout the CalDet running, it is important that the muons used are always of the same energies. Looking at the angular distribution of tracked particles from the CalDet at different times of the year and in various locations within the hall, the different muon sources can be identified. The track angles are calculated by fitting a straight line to all the accepted track hits. Figure 6.16 shows the angular distributions of tracks for the three running conditions of the CalDet:

- Cosmic muons, no beam muons, no PS muons (enclosure)

- Cosmic muons, no beam muons, PS muons (enclosure)

- Cosmic muons, beam muons, PS muons (beam area)

The spikes in the distributions at particular angles correspond to tracks in which all the hits line up precisely (for example, in sequential planes, strips $\mathrm{x}, \mathrm{x}+1, \mathrm{x}+2, \ldots$ are all hit). For these tracks, the angle measurement is limited by the width of the scintillator strips and a single topology could correspond to a range of angles. This is further indicated by a reduction of entries in the bins surrounding the spikes. For the purposes of placing a loose cut on the event angles, this does not pose a problem.

Figure 6.16 also shows the change in the distributions by making a cut on the vertical angle of the tracks with respect to the axis of the CalDet (i.e. perpendicular to the planes). The angular distributions of the three samples 
become comparable once tracks within $\pm 20^{\circ}$ of the axis have been removed. Thus, placing this cut on all muons used for strip-to-strip calibrations ensures consistency regardless of the running conditions. The response of the detector to these muons will be referred to as the Cosmic MEU.

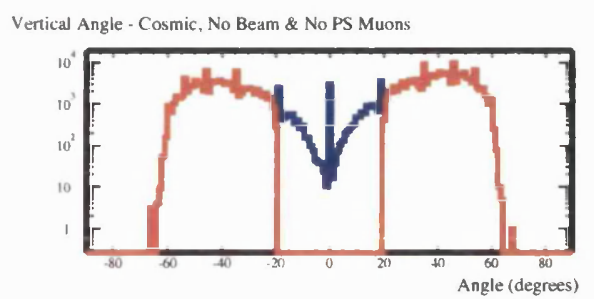

Verical Angle - Cosmic, No Beam \& PS Muons

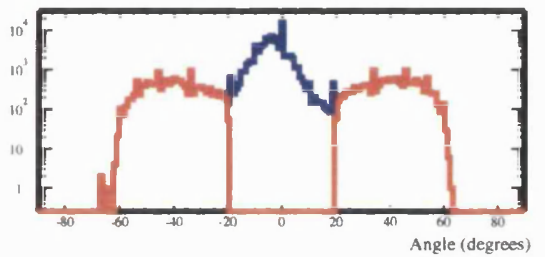

Verical Angle - Cosmic, Beam \& PS Muons

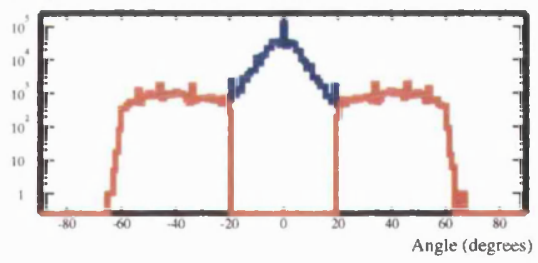

Horizontal Angle - Cosmic, No Beam \& No PS Muons

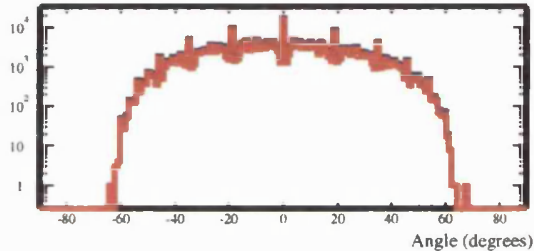

Horizontal Angle - Cosmic, No Beam \& PS Muons

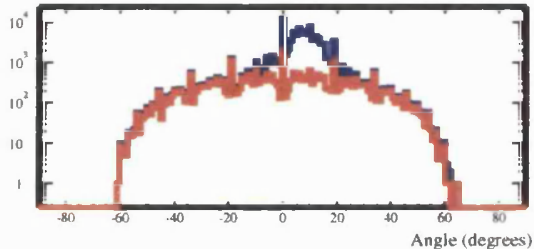

Honzontal Angle - Cosmic. Beam \& PS Muons

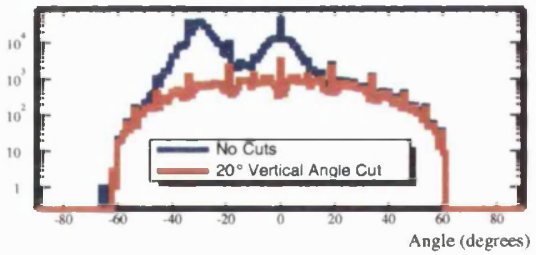

Figure 6.16: Angular distributions of muons in the three usual running conditions of the CalDet. Cutting on the vertical angle, a samples of comparable cosmic muons can be obtained.

Obtaining a sample of beam muons is more straight-forward. As can be seen from the angular distributions, beam muons are much more prevalent than the cosmic muons and, unlike the PS muons, are centered around $0^{\circ}$ in both horizontal and vertical angle. Therefore accepting only tracks that are within $\pm 10^{\circ}$ of the axis is sufficient to obtain a good sample. 


\subsubsection{Drift Point Corrections}

Every 20 minutes throughout data taking, the LI system pulses the LEDs at a single light level, illuminating every strip end. Comparing the response of the detector to the response of the PIN diodes gives a measurement of the gain drift during the run. This information is used to correct each accepted track hit to a reference point in the past for which the gain is well known. With this correction, any time dependence due to gain shifts in the phototubes or electronics is removed.

The importance of this correction to the strip-to-strip calibration can be seen by looking at the variation in gain over time. Figure 6.17 shows the average variation for all channels over a period of 2 days. The variation is at the level of $2 \%$ which directly translates to an error of $2 \%$ in the strip-to-strip calibration if these corrections are not applied.

The LI system also measures the linearity of the phototube response with light by measuring gain curves for each readout channel. The LI system pulses at a range of light levels, and the response in ADCs of each channel is plotted against the response of the PIN diode. Examples of these are shown in Figure 6.18. The drift points are then used to evolve the gain curves throughout the run and so, as demonstrated in the figure, the two gain curves taken at different times are equivalent after proper application of the drift point data.

The ability of the drift points to do this correctly is demonstrated in Figure 6.19. To make this figure, three gain curves are used. The first is used as a reference, the second measures the linearity a short time ( $\sim$ days) later, and the last measures the linearity again after an expected $\sim 20 \%$ gain change, (the High Voltage to all the phototubes was increased in the interim). The drift points are then used to evolve the reference gain curve to 
Gain Vs Time (Average of all channels)

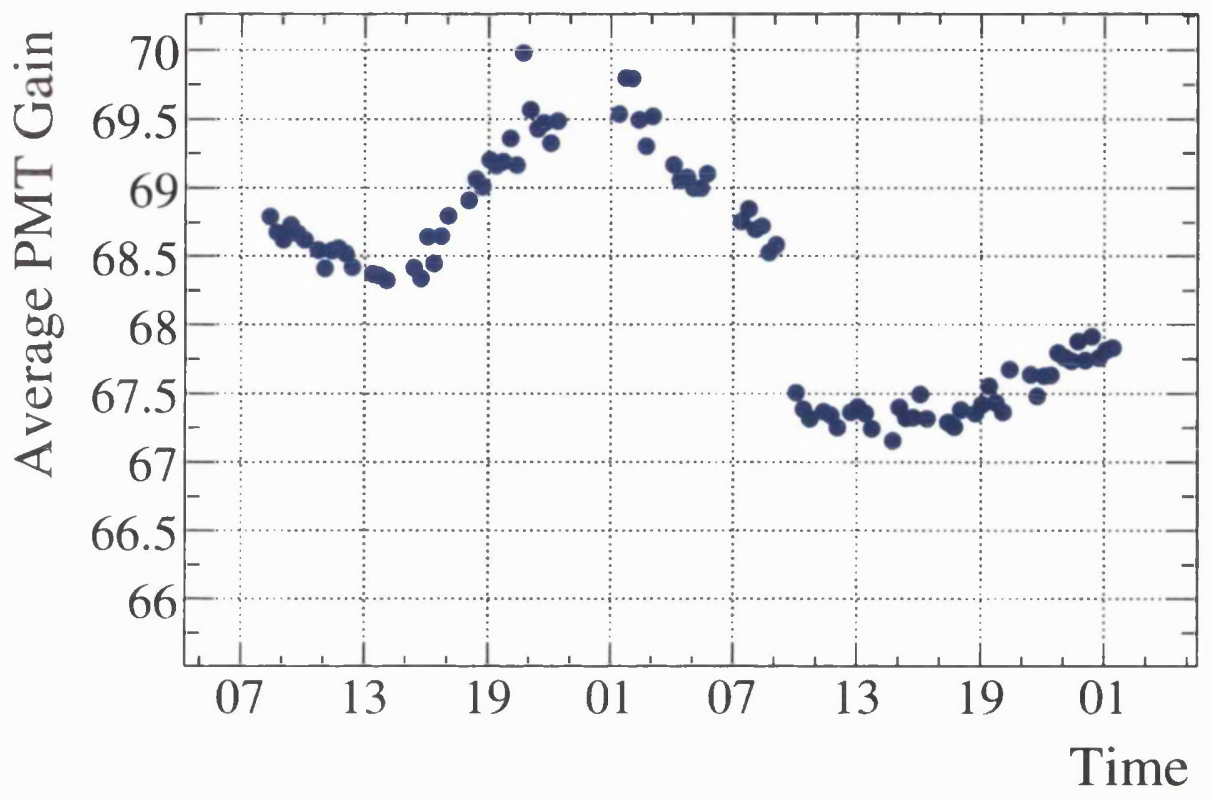

Figure 6.17: The average variation in gain for all phototube pixels over the period of $\sim 2$ days. The variations are typically of the order $2 \%$ on these timescales and can be accounted for using the LI system.

the later ones, and a different point along the curve is used to find residuals. The red histogram in Figure 6.19 shows the residuals for all channels before the drift point corrections are applied and the black histogram shows the residuals afterwards. The two peaks in the red histogram can be identified as the changes in gain between the reference time and the two later times. It can be seen from the black histogram that application of the drift point corrections removes the gain drift to better than $1 \%$. 


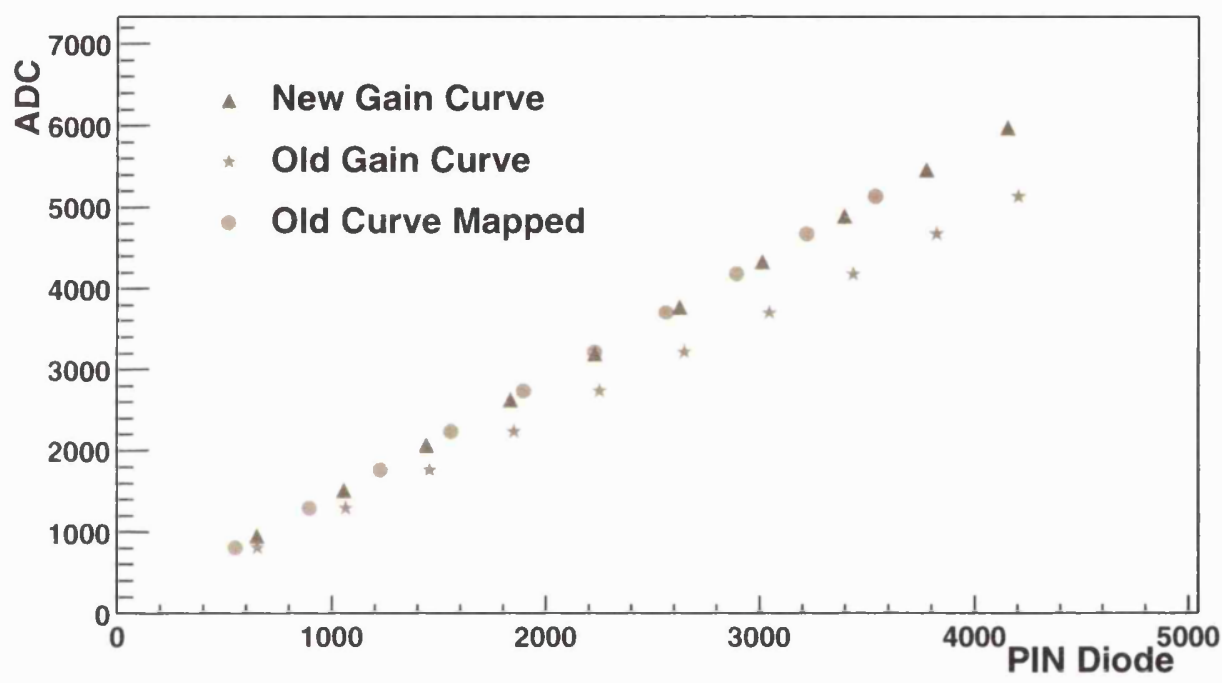

Figure 6.18: Gain curves showing the linearity of the phototube response with light level for a particular channel. The light level is measured by a PIN diode whose response is plotted on the y-axis. Two gain curves taken at different times are shown. The earlier gain curve is evolved using the drift point LI data to the later time after which it is shown to lie along the later gain curve. 


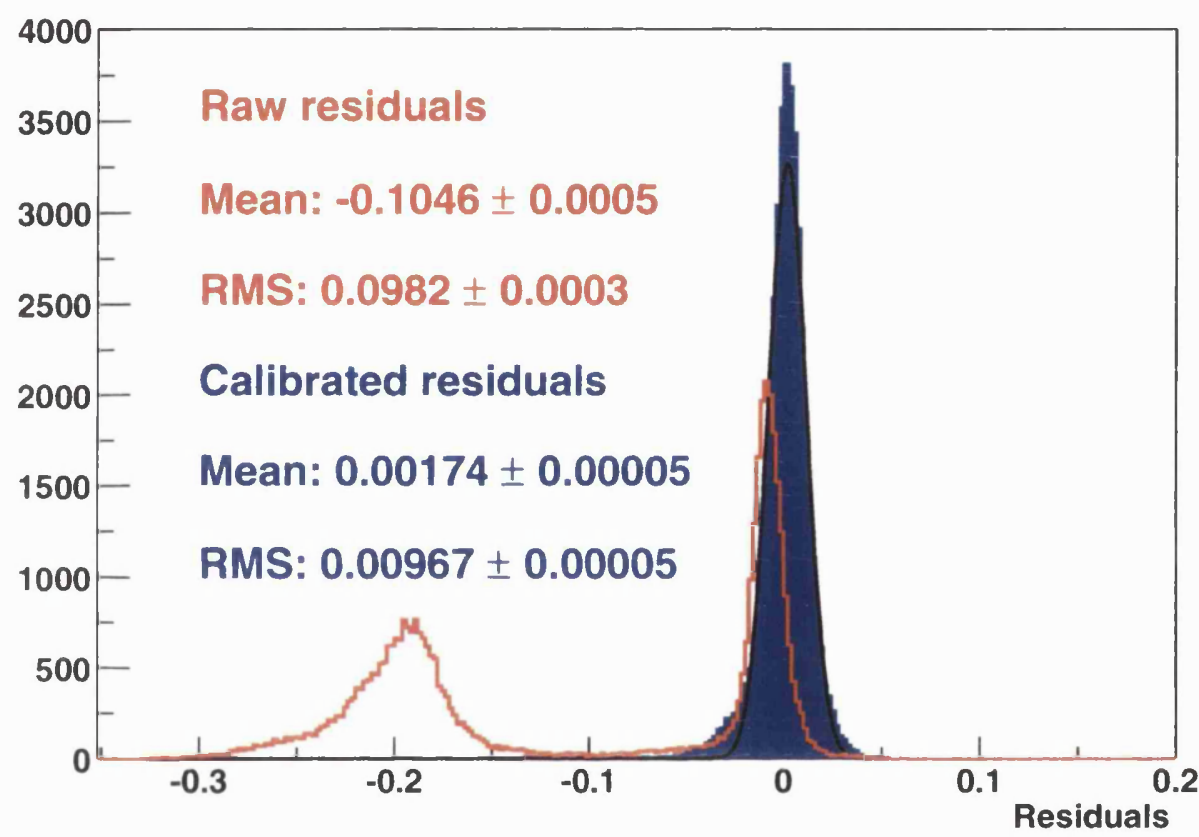

Figure 6.19: Demonstration of the ability of the LI system to correct for gain drift to better than $1 \%$. Three gain curves taken at different times are used to make this figure. The first is used as a reference and the LI drift point data is used to estimate the gain at the later times. The residuals from (expected - measured)/expected are plotted with (blue) and without (red) the drift corrections. The gain drift seen in the red histogram is accounted for in the blue to better than $1 \%$. 


\subsubsection{Angle Corrections}

As a muon passes through the scintillator, the amount of energy deposited is directly related to the amount of scintillator in its path. Therefore, particles traveling at higher angles with respect to the axis of the detector will typically produce hits with higher ADC counts. For calibration purposes, this angular dependence is removed by correcting all hits as if they had passed orthogonally through each plane.

In the case that the scintillator planes are not subdivided into strips, correcting for this effect is just a question of using the track angles to approximate the path-length at each plane and correcting the $\mathrm{ADC}$ value accordingly. However, the MINOS calorimeters use $4.1 \mathrm{~cm}$ wide strips of scintillator. This means that the path-length of a particle through a particular strip depends on the position that the particle entered that strip. This is illustrated in Figure 6.20 , where it can be seen that some particles traveling at higher angles will only clip the corner of a particular scintillator strip.

These corner clippers are very difficult to correct for on an event by event basis as the resolution afforded by the scintillator strips is not sufficient for such accurate tracking. Instead, an average path-length can be calculated for each track angle. Assuming that the distribution of particle entry position over the width of the strip is uniform, then the average path, $\langle d s\rangle$, is given by: 


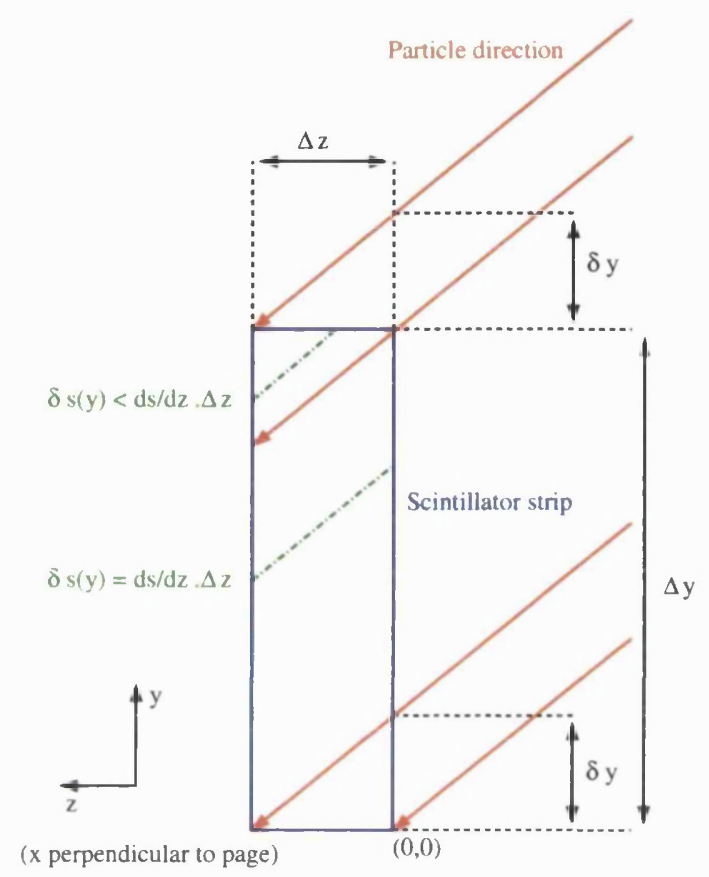

Figure 6.20: A schematic diagram illustrating the dependence of path-length through a scintillator strip on the angle of the track.

$$
\begin{aligned}
\langle d s\rangle & =\frac{\int_{0}^{\Delta y+\delta y} d s(y) d y}{\int_{0}^{\Delta y+\delta y} d y} \\
\langle d s\rangle & =\frac{\int_{\delta y}^{\Delta y} \frac{d s}{d z} \Delta z d y+2 \int_{\Delta y}^{\Delta y+\delta y} \frac{d s}{d z} \frac{d z}{d y} y d y}{\Delta y+\delta y} \\
\langle d s\rangle & =\frac{d s}{d z}\left(\frac{\Delta y}{\frac{\Delta y}{\Delta z}+\left|\frac{d y}{d z}\right|}\right)
\end{aligned}
$$

where $x$ is position along the length of the strip, $\mathrm{y}$ is position along the width of the strip and $\mathrm{z}$ is position along the axis of the detector; hence, $d s^{2}=d x^{2}+d y^{2}+d z^{2} . \delta y$ is the maximum y position for which the track will still pass through the strip. $\Delta y=4.1 \mathrm{~cm}$ is the width of a scintillator strip and $\Delta z=1 \mathrm{~cm}$ is the thickness. 
To apply this formula, the simplest approach is just to use the average angle for the whole track. However, sudden direction changes due to multiple scattering of the muon can often occur. Therefore, a better method is to calculate the local angle of the track at each plane separately using hits in nearby planes. Looking at Figure 6.21, the relationship between path-length and average measured ADC counts is shown for all hits in a sample of cosmic muons. The relationship after applying the path-length corrections is also shown. It can be seen that $\sim 60 \%$ variations in mean ADC are seen over the observed range. After applying the corrections, this dependency is removed to better than a few percent, although, at the very low path-lengths, it can be seen that both lines are discontinuous. This reflects the limited ability to make angle measurements because of the finite width of the scintillator strips.

Figure 6.21 also shows that there is still some dependence on the pathlength through the plane even after applying the correction. The approximation used in this analysis corrects for the largest effects, however there are other smaller effects which can also introduce a dependence. In particular, considering again Figure 6.20, at small and large values of $y$ the path of the particle through the strip is small and often no hit will be detected. Thus, it is incorrect to include these short path-lengths in the integral when calculating the average. Further, there is a dependence on $y$ which leads to an increase in the light detected with proximity of the track to the wavelength shifting fibre embedded in the scintillator strip. A proper treatment of these smaller effects is therefore necessary to completely remove the path-length dependence.

Projecting the points in Figure 6.21 onto the y-axis and weighting by the errors, Figure 6.22 shows that the corrections remove the ADC variations to 
$1.2 \%$ over the observed path-length range, which is adequate for the purposes of strip-to-strip calibration.

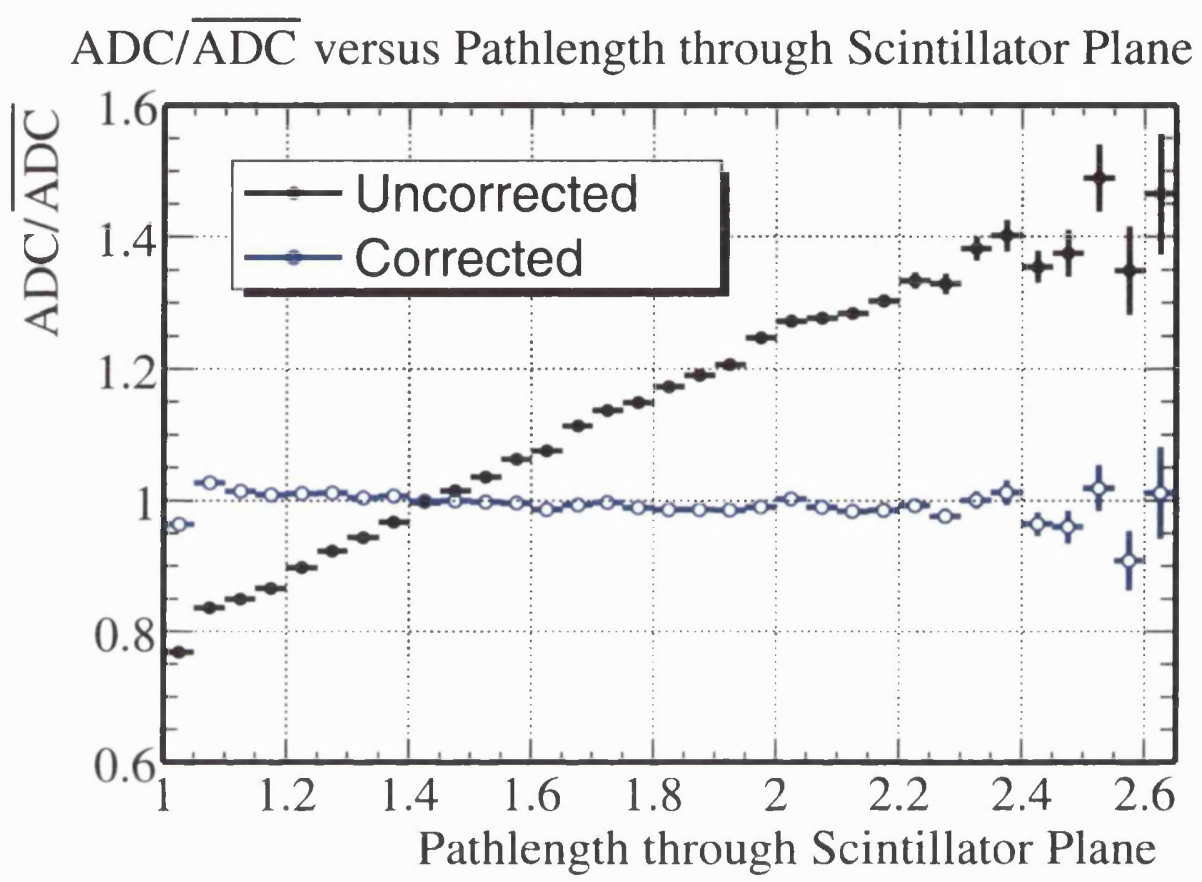

Figure 6.21: Normalized mean ADC as a function of path-length in the scintillator for a cosmic muon sample before and after the application path-length corrections.

\subsubsection{Attenuation Corrections}

The ADC value of a hit is also dependent on the position along the strip at which the particle crosses the plane. As a muon passes through a strip, some number of photons are captured by the WLS fibre and reemitted isotropically. The light is then attenuated as it makes its way to the phototubes due to absorption in the fibres. The magnitude of the signal at the phototube is therefore related to the amount of fibre between the muon crossing point and the phototubes. To account for this effect, all 


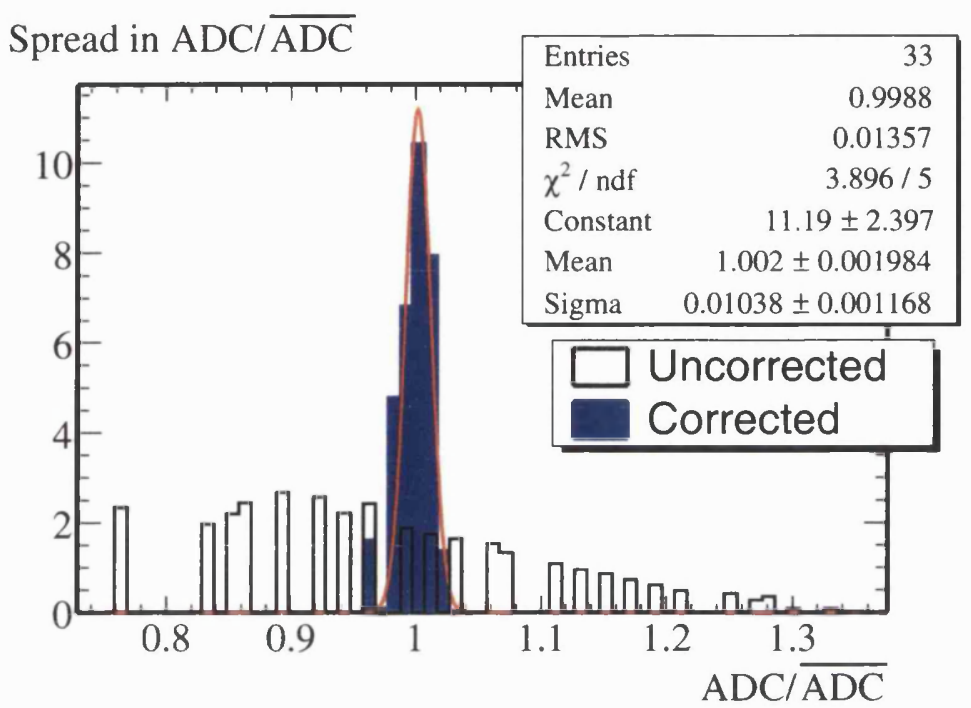

Figure 6.22: Variation in path-length corrected response. The points from Figure 6.21 are projected onto the $y$-axis and weighted by their errors. It can be seen that the applied correction flattens the response with path-length to $1.2 \%$.

reconstructed hits are corrected as if they had passed through the centre of the scintillator strip.

In order to apply a correction, the first step is to investigate the typical hit magnitude as a function of hit position along a strip. For a sample of cosmic muons, the drift point and angle corrections are applied and the average ADC value versus hit position along the scintillator strip are plotted. This is shown in Figure 6.23 for each end of the readout. It can be seen that the average slope is reversed, as expected, for the different sides of the readout. The observed signal reduction near the ends of the scintillator strip is due to the fact that there is no reflective coating at the ends of the strips. Light produced near the ends of the strips therefore passes out of the end of the scintillator and is absorbed by the manifold inside the module.

This dependence on position is characterized using 3 quadratic terms, 

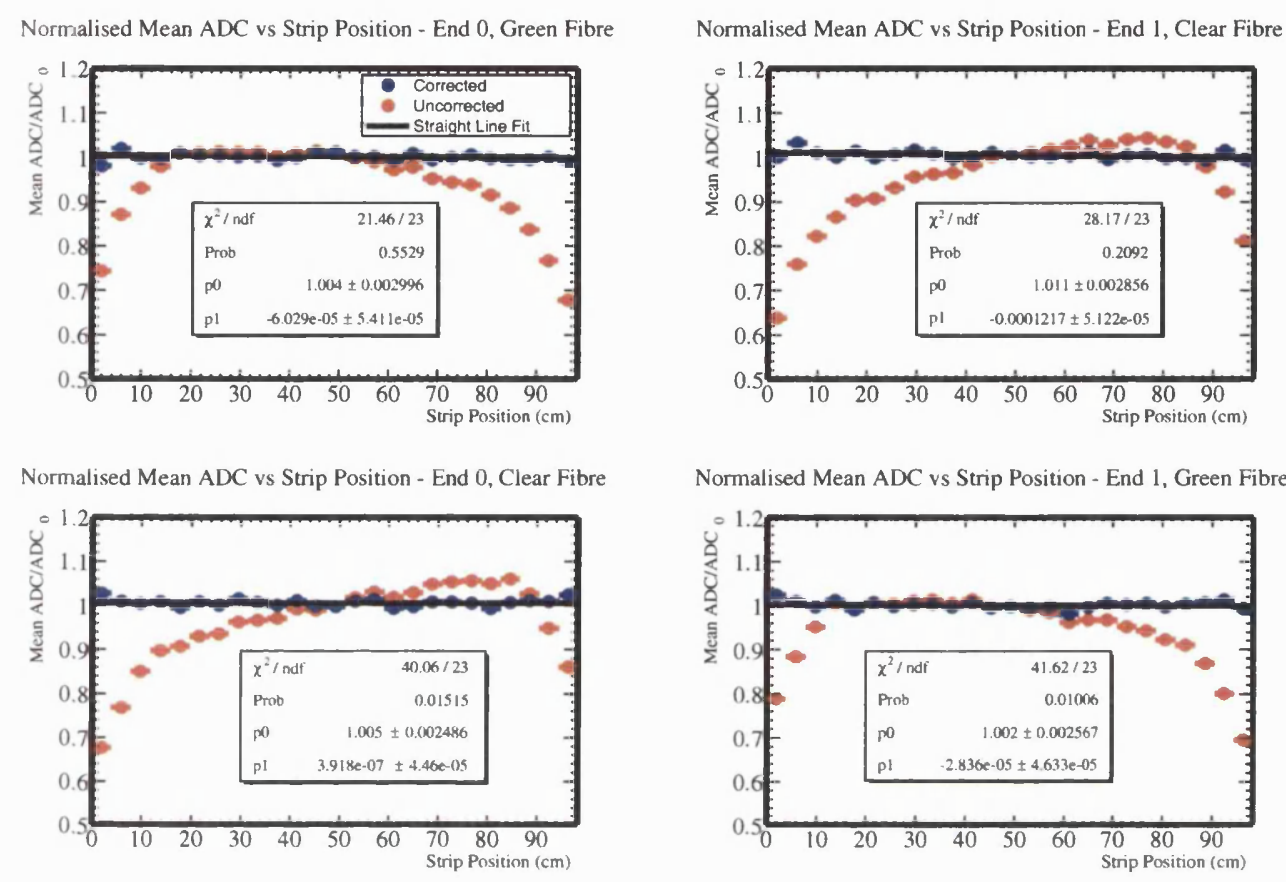

Figure 6.23: The dependence of muon pulse height with position along a scintillator strip with and without corrections. It can be seen that after corrections are applied the response is flattened to about $1 \%$.

one for each of the three different regions: the central region where the light output drops smoothly with distance from the readout connector and the two edge regions where the light ouput drops off sharply due to absorption by the manifold as described above. These terms are then used to correct all hits in all muon samples back to the centre of each strip. Also shown in Figure 6.23 is the attenuation corrected mean ADC value versus hit position along a strip. These points are fitted with a straight line in each of the four views. The $y$-intercepts and gradients from the fits are all approximately consistent with 1 and 0 respectively to approximately $1 \%$. 


\subsection{Results}

The calibration tools and procedures described in the previous sections are now applied to CalDet data. The following results summarize the detector response before and after calibration and show the success of the calibration procedures.

\subsubsection{Calibration Constants}

Using a cosmic ray muon sample, all necessary corrections are applied to the hits. For each strip end, the number of photoelectrons, corresponding to the $90 \%$ truncated mean, is calculated for a cosmic muon passing orthogonally through the centre of the detector. This value can be used to directly compare the light output and optical path efficiencies for all channels.

The calibration constants used for correcting the strip-to-strip variations are then simply these truncated means in terms of ADC counts. This single number for each channel removes all variations in detector response which do not significantly vary on short timescales.

\subsubsection{Uniformity of Detector Response}

The calibration constants remove both phototube gain and light output variations between the different strip ends. It is interesting to separate these two effects. Figure 6.24 shows the gains for the green and clear channels. The means of the two distributions are similar as expected and the RMS values indicate $\sim 20 \%$ variations in the gains.

The variations in light output for each strip-end in terms of photoelectrons are shown in Figure 6.25. The light output for the green and clear fibre readout channels are observed to differ by $\sim 25 \%$ and variations in light 

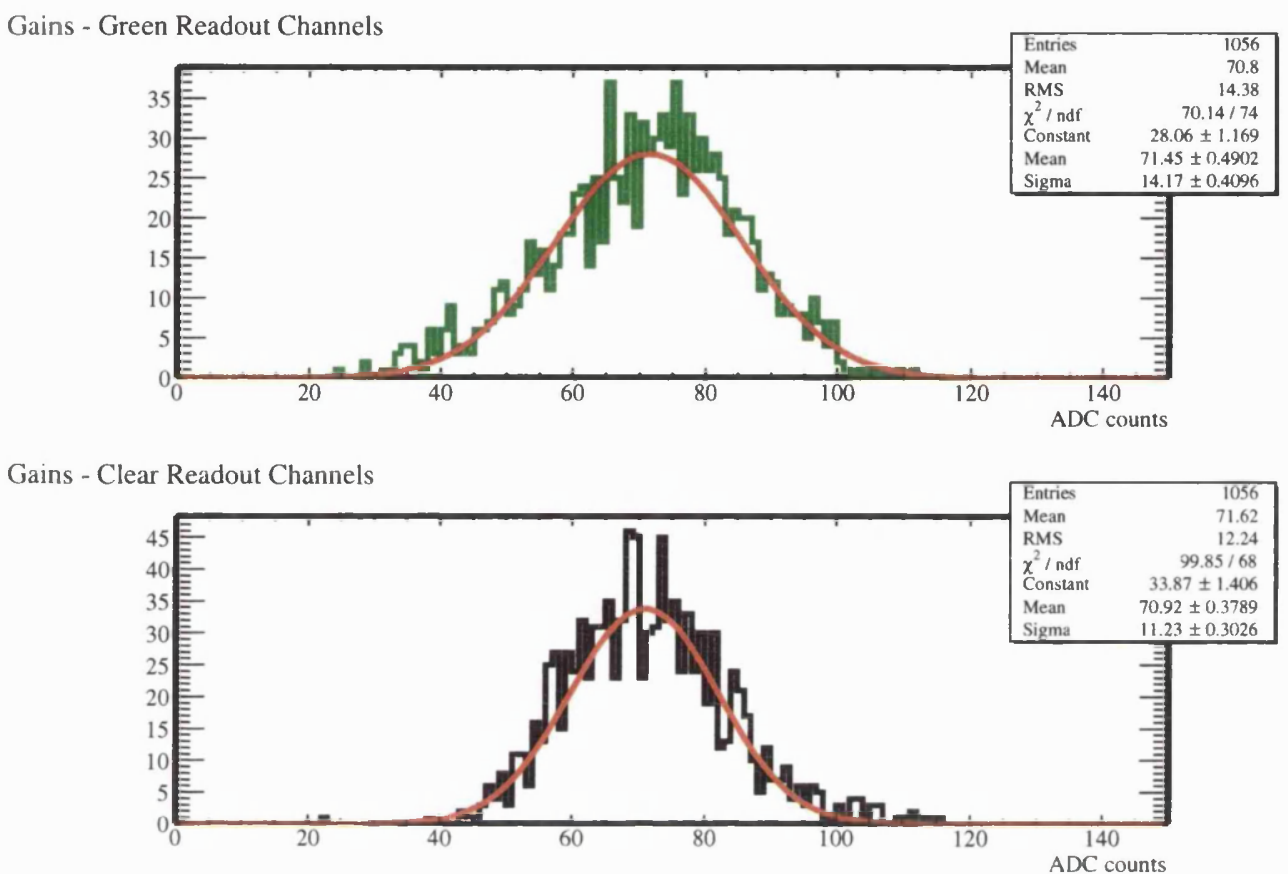

Figure 6.24: Variations in the phototube gain at the CalDet. Green and clear fibre readout channels are shown separately.

output for a particular set of channels are on the order of 10-15\%. Recalling again the observed gain variations of $20 \%$, this leads to variations in detector response in terms of $\mathrm{ADC}$ counts of $25-30 \%$ across the detector.

\subsubsection{Stability of Cosmic MEU over Short Time Scales}

Measured differences in the strip-to-strip calibration constants over time provide information about the stability of the detector response with changing conditions. Looking at short term variations, the effect of external environmental conditions can be monitored. Figure 6.26 shows the mean fractional difference in the calibration constants (with respect to the earliest set of constants) as a function of time for cosmic muons. The two distinct sets of points correspond to two different data taking runs taken about two weeks 


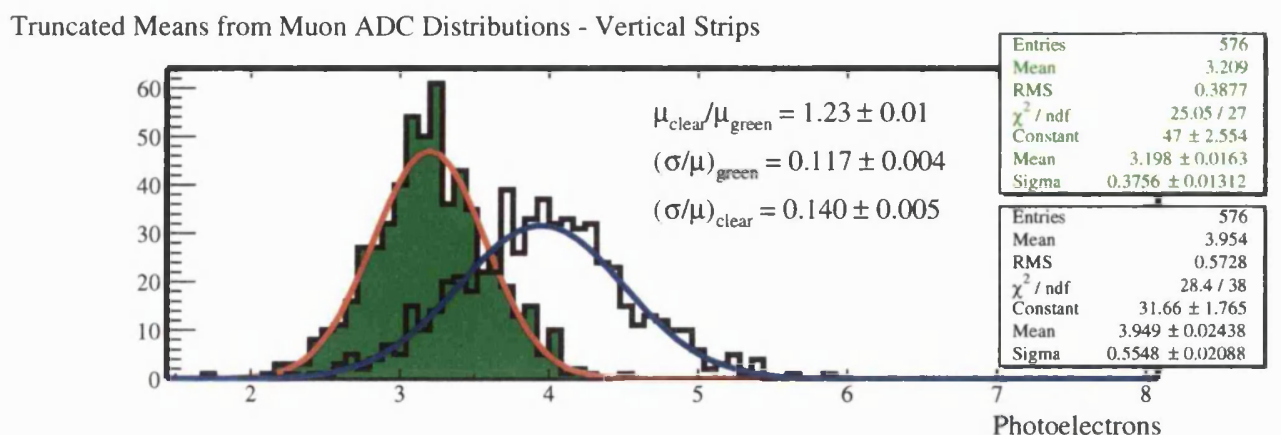

Truncated Means from Muon ADC Distributions - Horizontal Strips

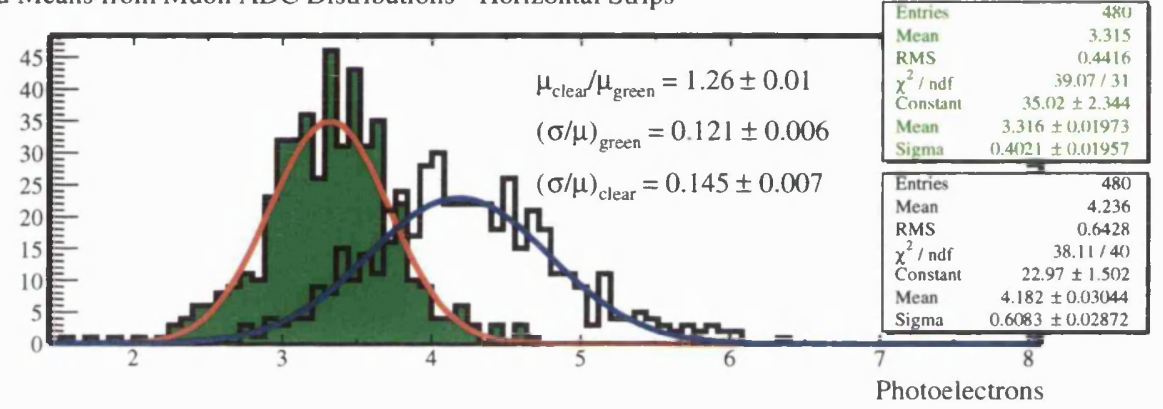

Figure 6.25: The light output of the CalDet readout channels in terms of photoelectrons. The mean light output for the green and clear channels are seen to differ by $\sim 25 \%$ and variations in light output observed for a particular set of channels is $10-15 \%$.

apart. The constants were updated approximately every 3 hours during the runs.

It can be seen that there are short term variations within a run on the order of $1 \%$ as well as an overall difference between the two runs of approximately $2 \%$. This is a potentially worrying finding as it implies that without accurately calibrating the entire detector on these time scales, accurate calibration to $2 \%$ may not be attainable. At CalDet, calibrating at this frequency is possible as there are always many muons passing through the detector. However, at the Far Detector there are only an estimated 500 muons passing through each strip per month. It is therefore important to 
Percentage Shift in Mean MEU versus Time

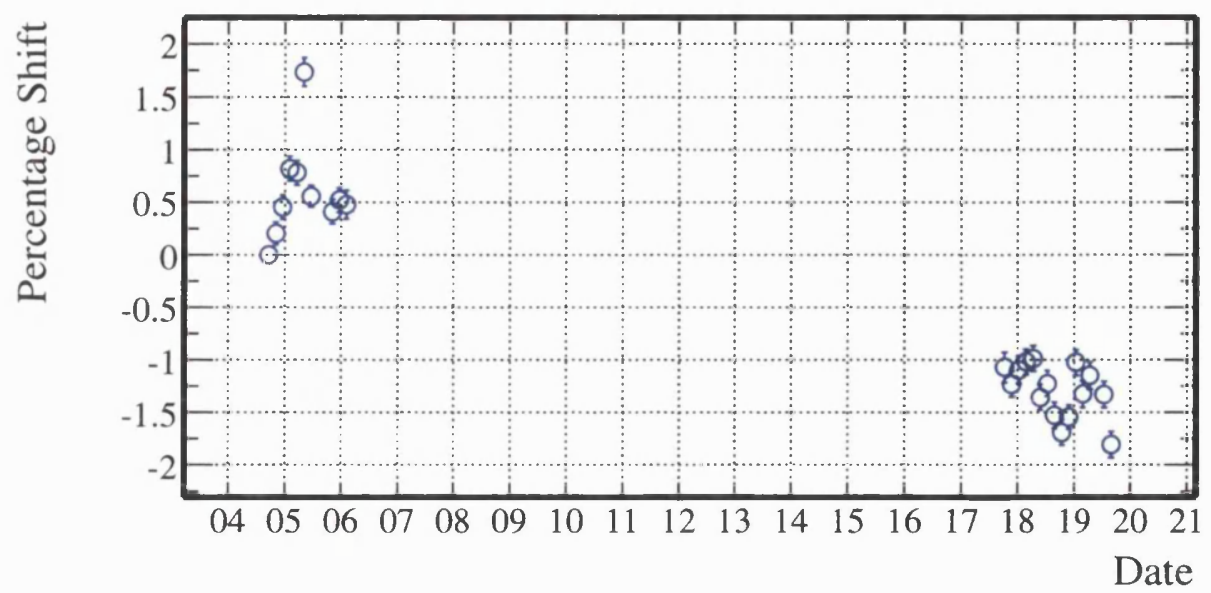

Figure 6.26: Variations in calibration constants over a two week period. Differences of $2 \%$ in the mean MEU for all strips are observed on these time scales which will impact the accuracy of the calibration.

discover the source of these changes in order to estimate the impact on Far Detector calibration.

\section{Temperature Effects}

Given the pattern of the changes in Figure 6.26, an obvious culprit is the changing environmental conditions, specifically temperature. At CalDet, the temperature is measured using a probe which records the ambient air temperature in the vicinity of the detector every minute. These measurements are then averaged to give hourly readings.

Previous measurements have shown that scintillator light output drops with increasing temperature with a constant of proportionality: $\sim-0.3 \%$ for $\Delta T=+1^{\circ} \mathrm{C}[61]$. Using this relation, Figure 6.27 shows the expected variations in scintillator light output due to temperature. The observed shifts are also plotted for comparison and it can be seen that the trends agree fairly 
well. In terms of the Far Detector, this is encouraging as the temperature conditions in the Soudan mine should be very stable only fluctuating a few degrees throughout the year.

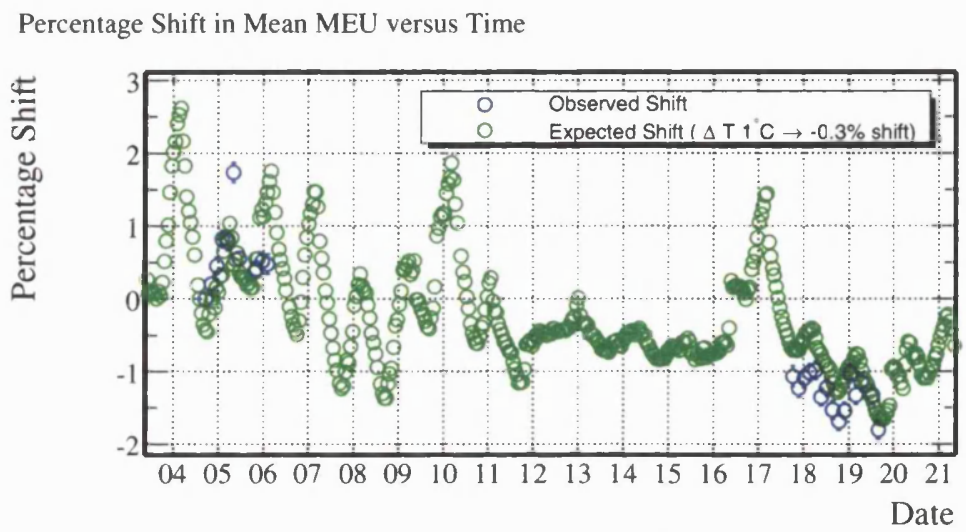

Percentage Shift in Mean MEU versus Time

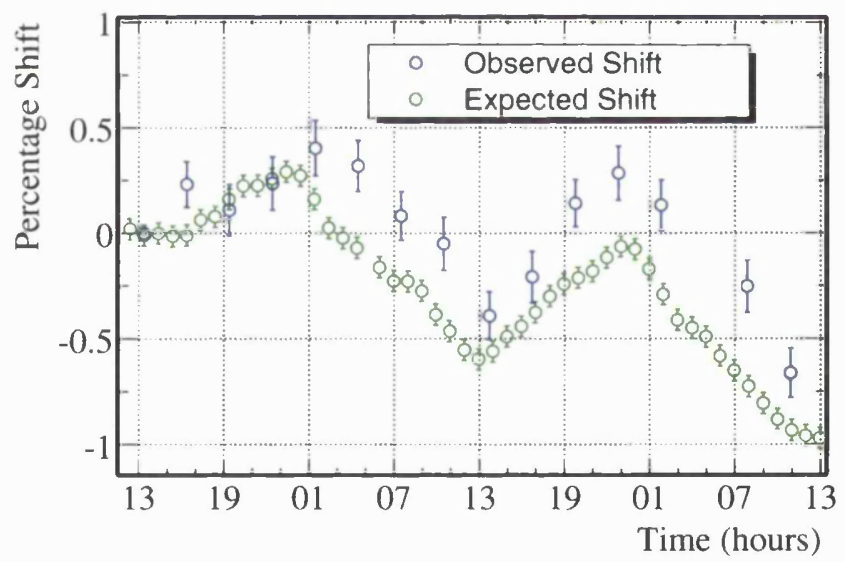

Figure 6.27: Observed shifts in calibration constants plotted with those expected due to local temperature variations. The upper plot shows the pattern over the entire two week period, whereas the lower plot shows the hourly variations for the later of the two data sets. 


\subsubsection{Application of Strip-to-Strip Calibration}

The success of the calibration procedure can be tested by applying the corrections to a different muon sample and observing the hit distributions. Figure 6.28 shows the strip characterization in terms of cosmic MEUs for each of the 4 readout views. For a correctly calibrated detector, these distributions should each have a mean of 1 and a width given by the errors on the calibration constants. It can be seen that the distributions peak at 1 within errors and the widths are $\sim 6 \%$ reflecting errors on the calibration constants of $\sim 4 \%$ for these muon samples.
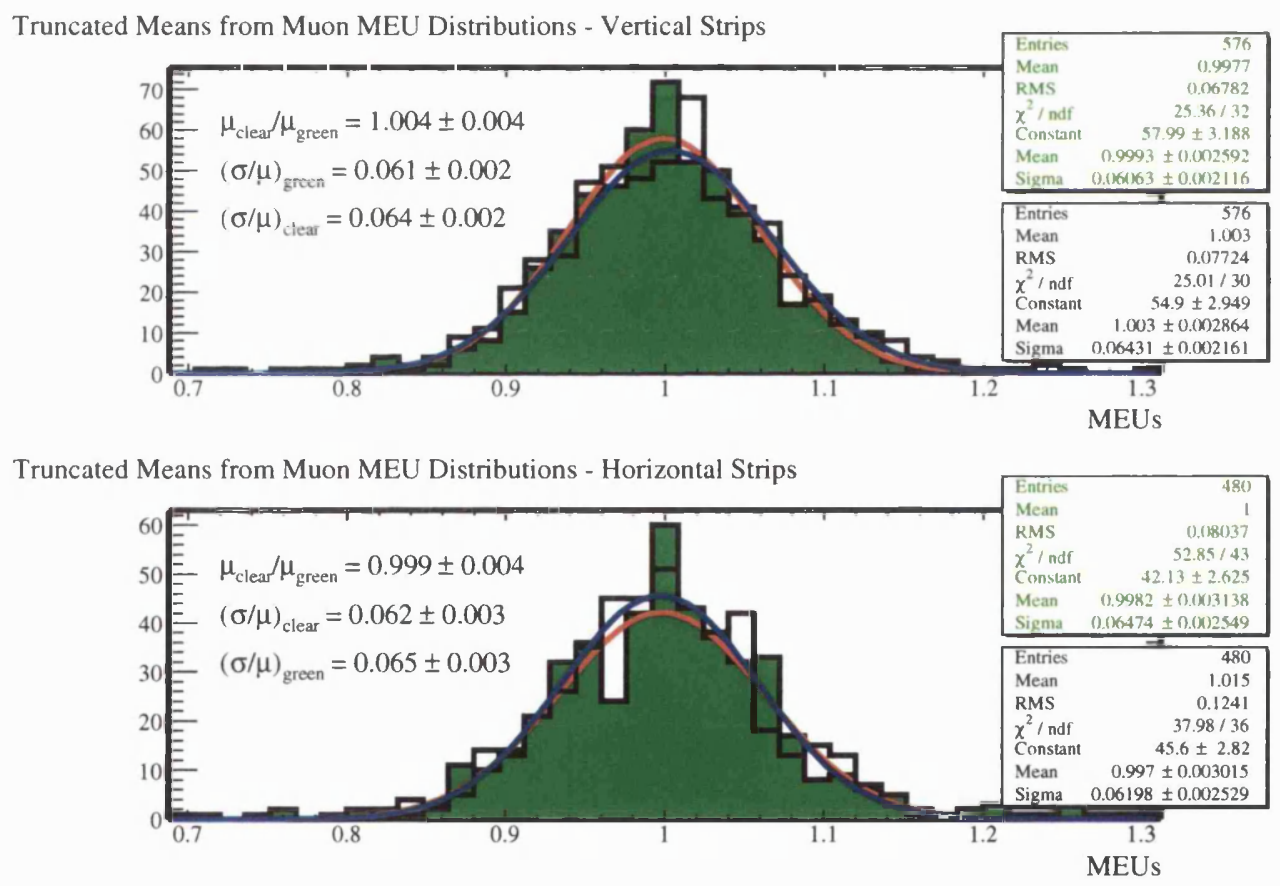

Figure 6.28: The result of strip-end characterization for a muon sample corrected for strip-to-strip variations. The distributions peak at 1 corresponding to the fact that the strip-end variations are removed by the strip-to-strip calibration procedure. 
A further test is to look at a distribution of the average muon hit value per event for the green and clear fibre readout channels separately, with and without calibration. Without calibration, differences between the green and clear readouts are expected and observed as shown in the upper plot of Figure 6.29. These differences are observed to be $\sim 25 \%$, in keeping with the differences in the calibration constants. The distributions are not Gaussian as might be expected, since large energy deposits in a single strip from the Landau tail can dominate the mean $\mathrm{ADC}$ value in an event, causing the distribution to be skewed to higher values. Once calibration is applied, the distributions converge as shown in the lower plot of Figure 6.29, such that the means agree to better than $2 \%$, as required for relative calibration.

The calibration constants have also been applied to samples of electrons. Figure 6.30 shows the summed ADC distributions for a sample of $2 \mathrm{GeV}$ electron events in each of the four views. Once again, large differences in the means are seen between the green and clear readout channels. Once calibration is applied, Figure 6.31 shows that the distribution means converge at the $2 \%$ level. The widths get narrower, but only by a few percent, since the main contribution to the widths is from the intrinsic resolution afforded by the calorimeter. 

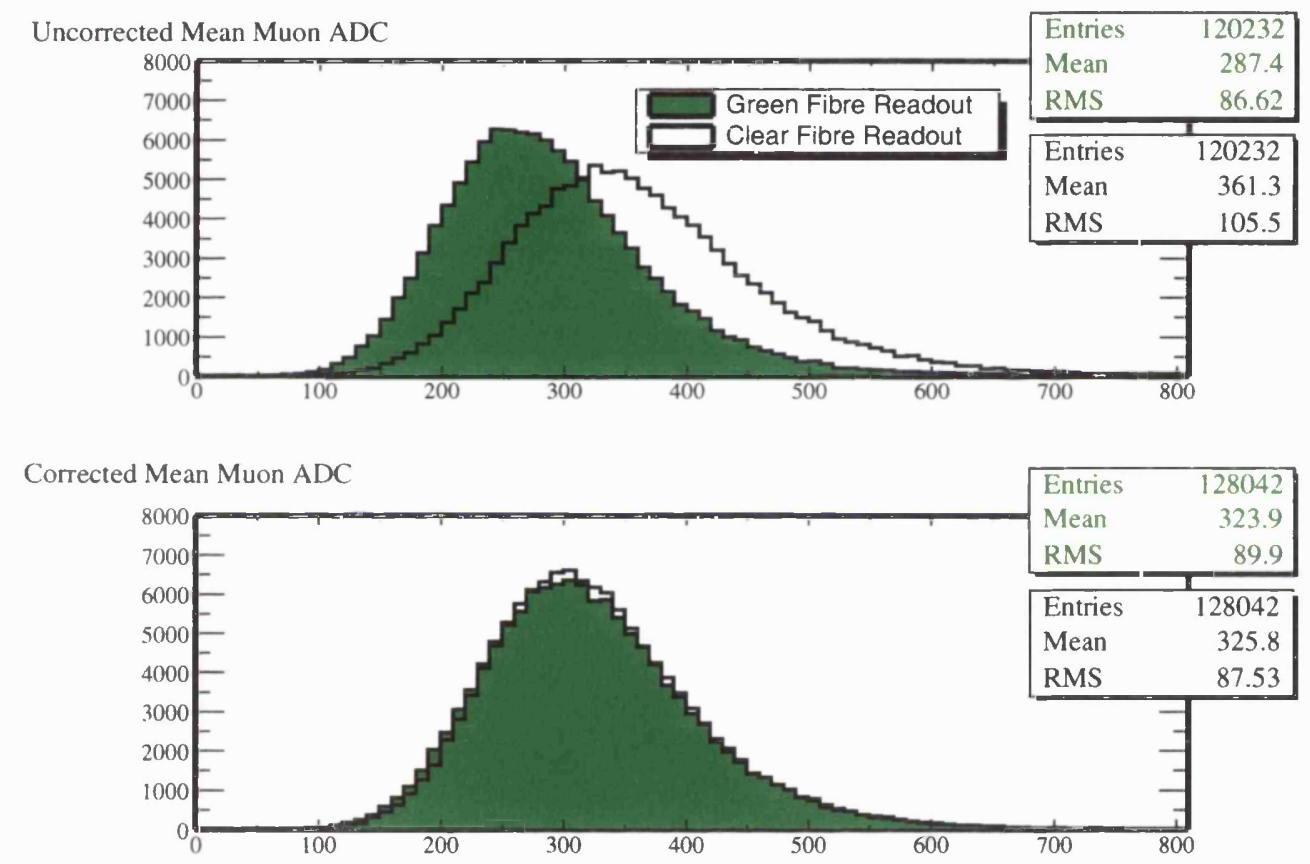

Figure 6.29: The mean muon hit response in ADCs before (upper) and after calibration for the green and clear fibre readout channels. The calibration corrects the distributions such that the means agree to $2 \%$ as required for relative calibration. 
Uncorrected Electron Summed ADC Distributions - Vertical Strips

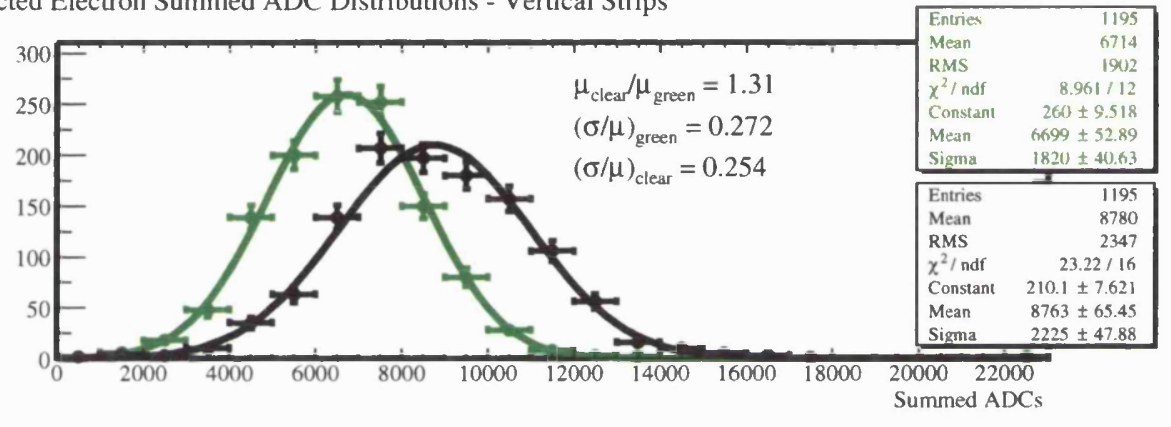

Uncorrected Electron Summed ADC Distributions - Horizontal Strips

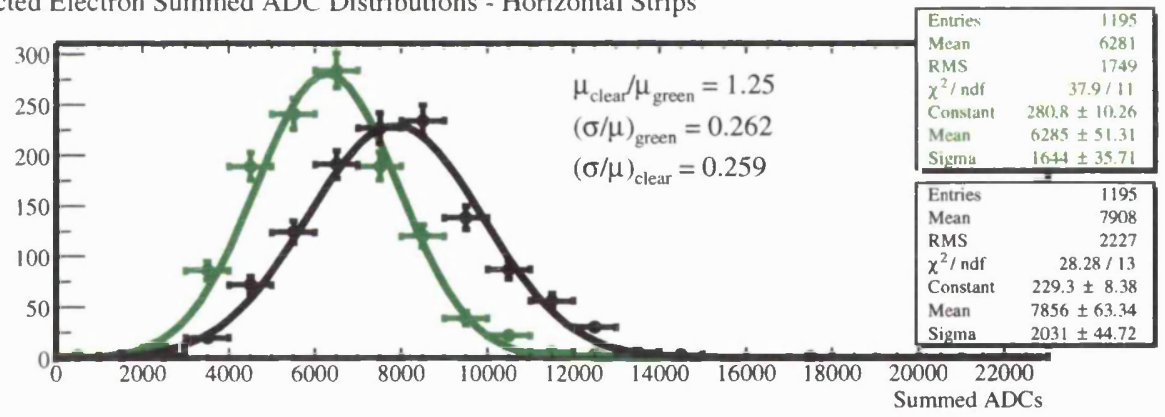

Figure 6.30: The summed ADC distributions for a $2 \mathrm{GeV}$ electron sample without calibration. The four different histograms correspond to the green and clear readout channels for the vertical and horizontal strips. 

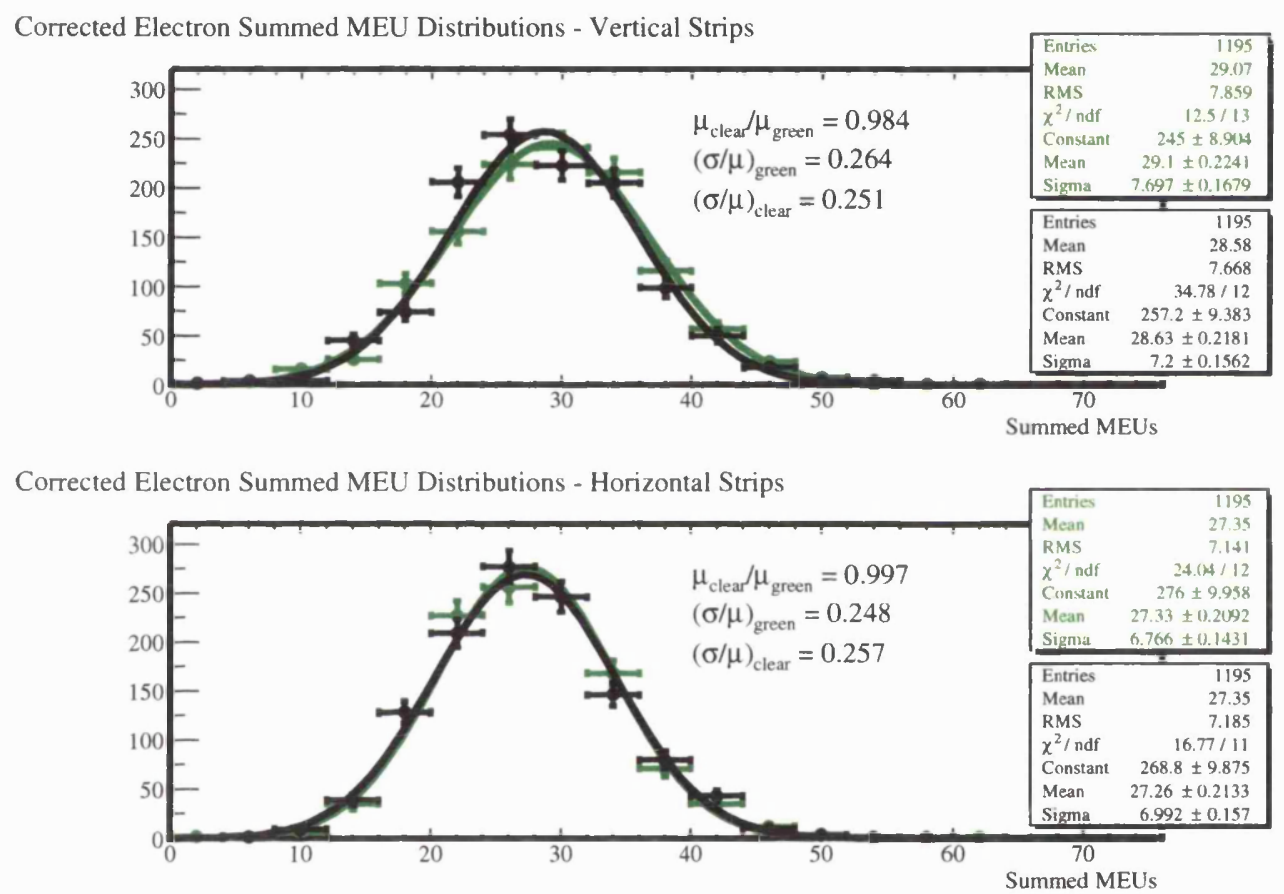

Figure 6.31: The summed MEU distributions for a $2 \mathrm{GeV}$ electron sample after strip-to-strip calibration. The differences between the green and clear readout channels for both sets of strips are removed to the $2 \%$ level. 


\subsubsection{Beam Muon Characteristics}

Beam muon events are primarily identified by a track which is within $10^{\circ}$ of the beamline in both orientations. However, as discussed in Section 6.2.4, particularly at lower energies, pions are also found by the tracking algorithm. Further, the muons in the test-beam result from pion decay, therefore within the selected sample there is also a range of muon energies. This is illustrated in the stopping plane plot shown in Figure 6.7.

The CalDet will measure the energy loss for pions, protons and electrons and express them in terms of some energy scale defined by muons. A way to set a scale for the absolute calibration, is to use the response of a sample of muons with well known energy in the CalDet. The other MINOS detectors can then use the measurements from CalDet by isolating a similar sample of muons.

One way to obtain a sample of muons with known energy at CalDet is to assume that those particles in the peak of the last hit plane distribution are muons at the beam energy. Using this method, the stopping plane has been plotted for a range of beam energies. This is shown in Figure 6.32, and it can be seen that there is a linear relationship between the two quantities as expected.

Also shown in Figure 6.32 is a plot of predicted range in iron versus muon momentum, (made using the tables from [62]). It can be seen that the gradients are similar for the two plots, although the actual muon range is significantly lower in the CalDet measurements. This discrepancy may be due to a number of reasons: in the iron plot, energy losses in the scintillator have not been accounted for, although this is expected to be a small effect; the true energy distribution of the muons in the peak is not known well, since an accurate simulation of the beam-line is not available; also pion contamination 
in the sample may broaden the peak in the stopping plane distribution and reduce the mean by contributing to the low side tail.

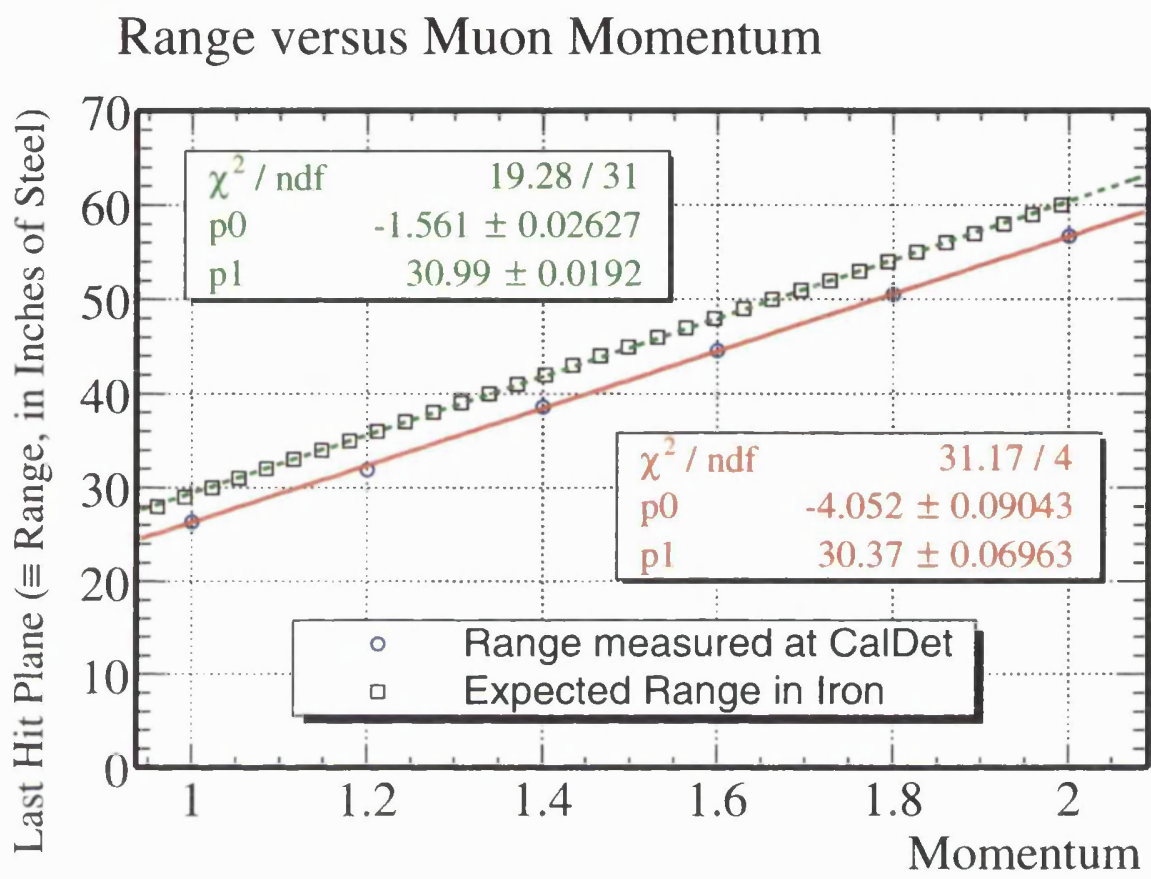

Figure 6.32: Range versus beam momentum for stopping muons in the CalDet. Also shown in the expected range of muons in iron from tables published in [62].

To investigate muon $\mathrm{dE} / \mathrm{dx}$, the strip-to-strip corrected response of the detector for a sample of stopping muons can be used. From a $2 \mathrm{GeV}$ beam run, the events which stop under the peak have been selected. The hits from the events are corrected using the strip-to-strip calibration constants obtained from a sample of cosmic ray muons. The hits are histogrammed for each strip-end and the $90 \%$ truncated means of the distributions are found as usual. For each two plane section of the detector, the mean of the strip-end truncated means is found and has been plotted in Figure 6.33.

It can be seen that there is a significant increase in light output near 
Change in Light Output with Plane for $2 \mathrm{GeV}$ Muons

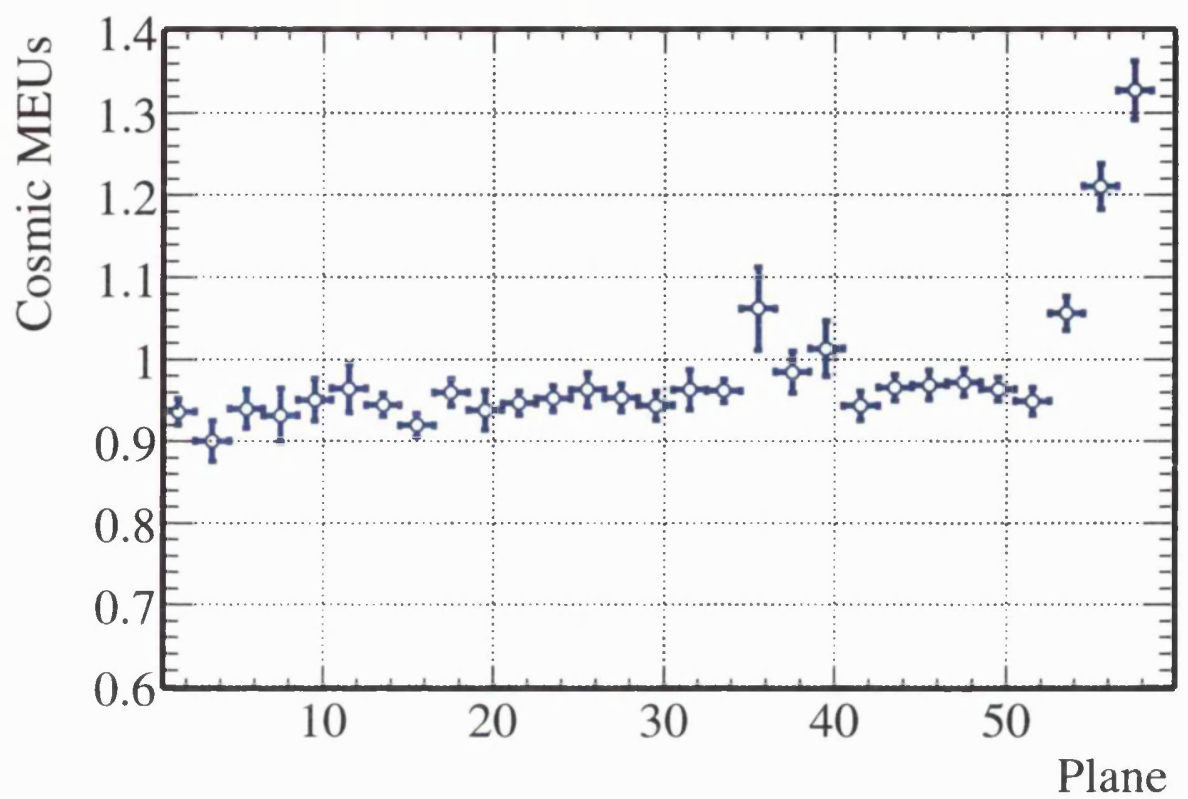

Figure 6.33: Muon $\mathrm{dE} / \mathrm{dx}$ versus plane for $2 \mathrm{GeV}$ beam muons.

the end of the detector, corresponding to the muons stopping. The average amount of energy deposited at the front of the detector is $\sim 0.93$ MEUs, where $1 \mathrm{MEU}$ corresponds to the average energy deposited by a muon from the cosmic ray sample. (The beam muon value is not $1 \mathrm{MEU}$ because the cosmic ray muons have an average energy of $\sim 4 \mathrm{GeV}$ at the Earth's surface [9] whereas the beam muons are at $2 \mathrm{GeV}$. Further, the tracking requires at least 8 hit planes and so muons traveling at small zenith angles are not selected. This also has the effect of increasing the average particle energy of the cosmic ray muon sample.) A possible value to use for the absolute calibration scale is therefore the number of photoelectrons corresponding to $0.93 \mathrm{MEUs}$. The response of electrons and hadrons of different energies can then be expressed in terms of the response of $2 \mathrm{GeV}$ muons. 
Using muons from the beam to set the scale for the absolute calibration is an attractive idea as the beam provides a source of particles at an approximately constant energy, traveling orthogonally through the centre of the detector. However, obtaining a good sample of muons at the nominal beam energy by using the stopping plane distribution may be misleading without a better understanding of the beamline. Therefore, rather than trying to isolate particles of known energy from the beam, the calibrated response of muons at some arbitrary, but constant, distance from the end of the track may be more appropriate for setting the scale for the absolute calibration.

\subsection{Summary}

In this section, tools developed for carrying out calibration at CalDet are presented and results from their application shown. Variations in channel response of $\sim 25 \%$ have been removed using strip-to-strip calibration to the $2 \%$ level. This has been demonstrated both using muon and electron event samples.

Stability measurements have shown that the detector response is stable to a few percent over periods of weeks. The variations in light output observed were shown to correlate strongly with temperature indicating that in a more stable environment, such as the Soudan mine, the detector response should be significantly more stable with time.

Measurements of range and $\mathrm{dE} / \mathrm{dx}$ for beam muons have also been carried out with regards to setting a scale for the absolute calibration of the CalDet. 


\section{Chapter 7}

\section{Extraction of Neutrino}

\section{Oscillation Parameters}

The goal of the MINOS experiment is to find evidence for neutrino oscillations using a $\nu_{\mu}$ beam and to measure the associated oscillation parameters. To be confident that the measured parameters are accurate, it is important that consistent results are obtained from independent analyses. In Section 4.3, the measurements expected to be most sensitive to the expected oscillation signal have been described; namely the T-test (the ratio of ratios: $\left.(N C / C C)_{n e a r} /(N C / C C)_{f a r}\right)$ and the comparison of the $\nu_{\mu} \mathrm{CC}$ energy spectra from the Near and Far detectors. These measurements rely on accurate reconstruction of neutrino interactions in the detectors and, in particular, the absolute energy calibration.

In this chapter, results from a study investigating the measurement potential of a different analysis channel are presented. Extracting the oscillation parameters using only the reconstructed muon energy from CC events has been considered. The advantage of this technique is that the muon energy can be found either from range, if the muon stops in the detector, or from 
curvature in the magnetic field; the measurement is therefore independent of the energy calibration of the detectors.

The measurement does depend on the Near/Far spectra differences which arise due to the divergence of the beam between Fermilab and the Soudan Underground Lab. MINOS measurements which are sensitive to this difference will ultimately rely on Monte Carlo in order to extract the oscillation parameters. A number of secondary hadron production models exist which can be used to predict the primary pion divergence and ultimately the neutrino fluxes. A comparison of these models can be used to estimate the dependence of the physics measurement on the uncertainty in the neutrino fluxes. Also, assuming that one of the models is correct, the sensitivity of measurements to the oscillation parameters can be estimated.

\subsection{Beam Neutrino Flux}

Interactions of the protons from the Main Injector with the NuMI carbon target are simulated using four different hadron production models. The models differ in their predictions of the abundances and transverse momenta of the secondary pions and kaons produced. For each model, the pions and kaons are subject to the effects of the magnetic focusing horns in the Low Energy beam configuration, which attempt to produce a parallel beam. Subsequently, the secondaries decay in the $675 \mathrm{~m}$ long evacuated pipe or are absorbed further downstream. The neutrinos resulting from the decays of these pions and kaons constitute the beam.

The neutrino flux from the models is expressed in terms of \# $\# / \mathrm{m}^{2} / P O T$ as a function of energy, (where POT is the number of Protons incident On the NuMI Target). To calculate the neutrino event rates at the Near 
and Far detectors, the neutrino flux is weighted by exposure (the combination of POT and the detector mass) and by the neutrino cross-section to give \#events/kt/yr as a function of energy. The four different hadron production models used are GFLUKA [63], BMPT [64], MARS [65] and MALENSEK [66]. The predicted Near and Far $\nu_{\mu}$ CC event rates for each of these models are shown in Figure 7.1 assuming no oscillations. Differences in the expected energy spectra of up to $15 \%$ between the models can be seen, especially at the peaks of the distributions.

Since MINOS will make measurements by comparing the Near and Far spectra, a useful quantity to know is the normalized $\nu_{\mu} \mathrm{CC}$ event rate Far/Near ratio as a function of energy without oscillations. This is shown in Figure 7.2 for each of the four hadron production models. In this figure it can be seen that differences of up to $30 \%$ are observed at the lower energies between the four models, however, these are in regions where the rate is negligibly small. In the regions where the rate is highest, differences of up to $5 \%$ can be seen. For this reason, the MIPP hadron production experiment at Fermilab will measure the pions and kaons as they are produced from the NuMI target. The results will be used to tune the models in order to reduce the uncertainties in the Near/Far comparison.

Despite the uncertainties in the Near/Far ratio at present, these models together can be used to estimate the measurement sensitivity. The predicted sensitivities reflect the possible scenarios in the real measurement and so provide an estimate of the best and worst case. 


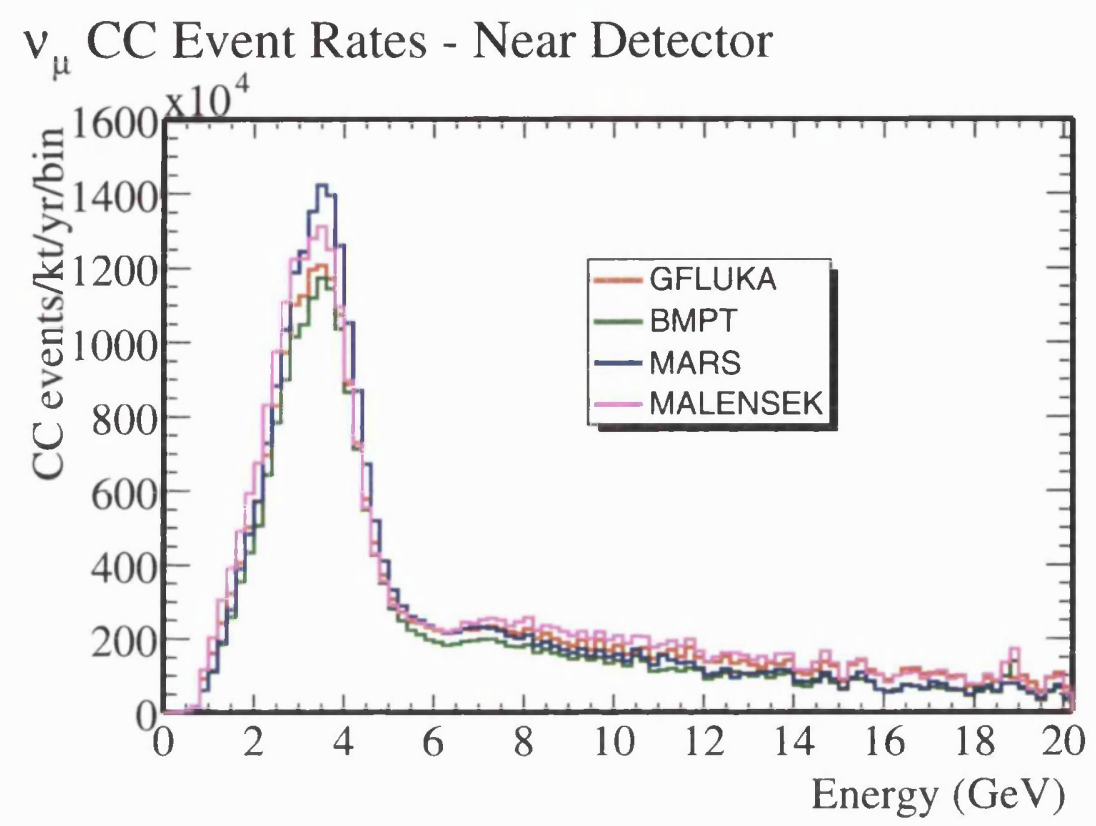

$v_{\mu}$ CC Event Rates - Far Detector

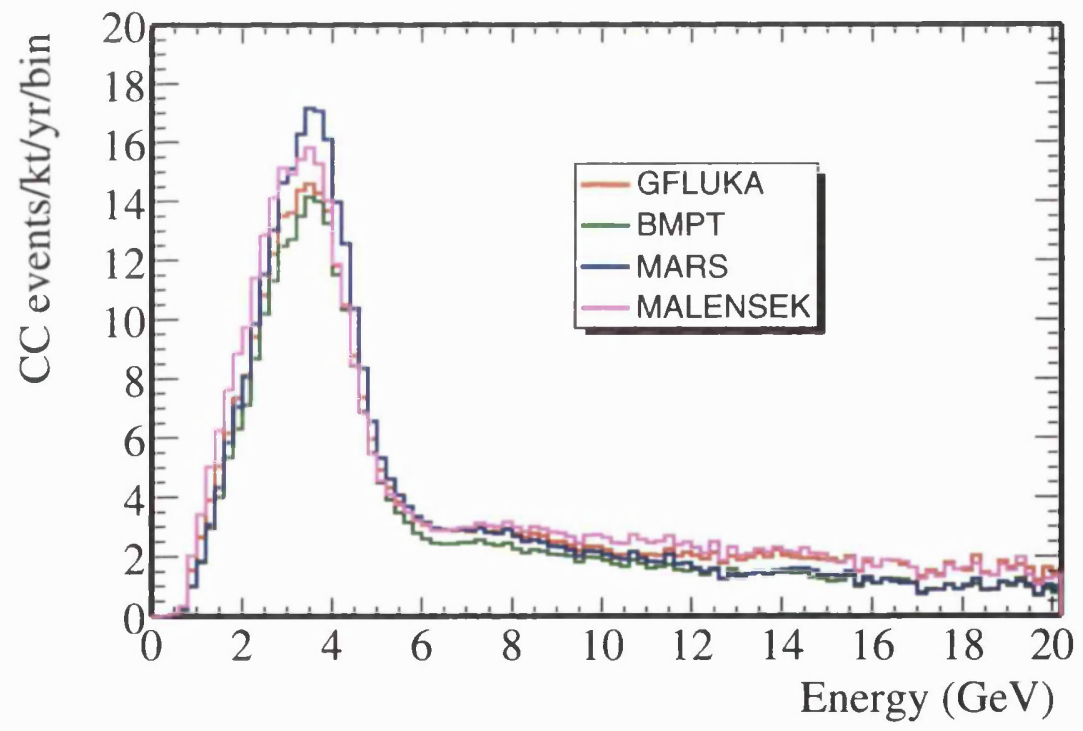

Figure 7.1: The predicted Near (upper) and Far $\nu_{\mu}$ CC event rates calculated using four different hadron production models, (no oscillations). Some differences between the histograms can be seen especially in the peaks of the distributions. 
Far/Near Normalised Event Rate Ratio

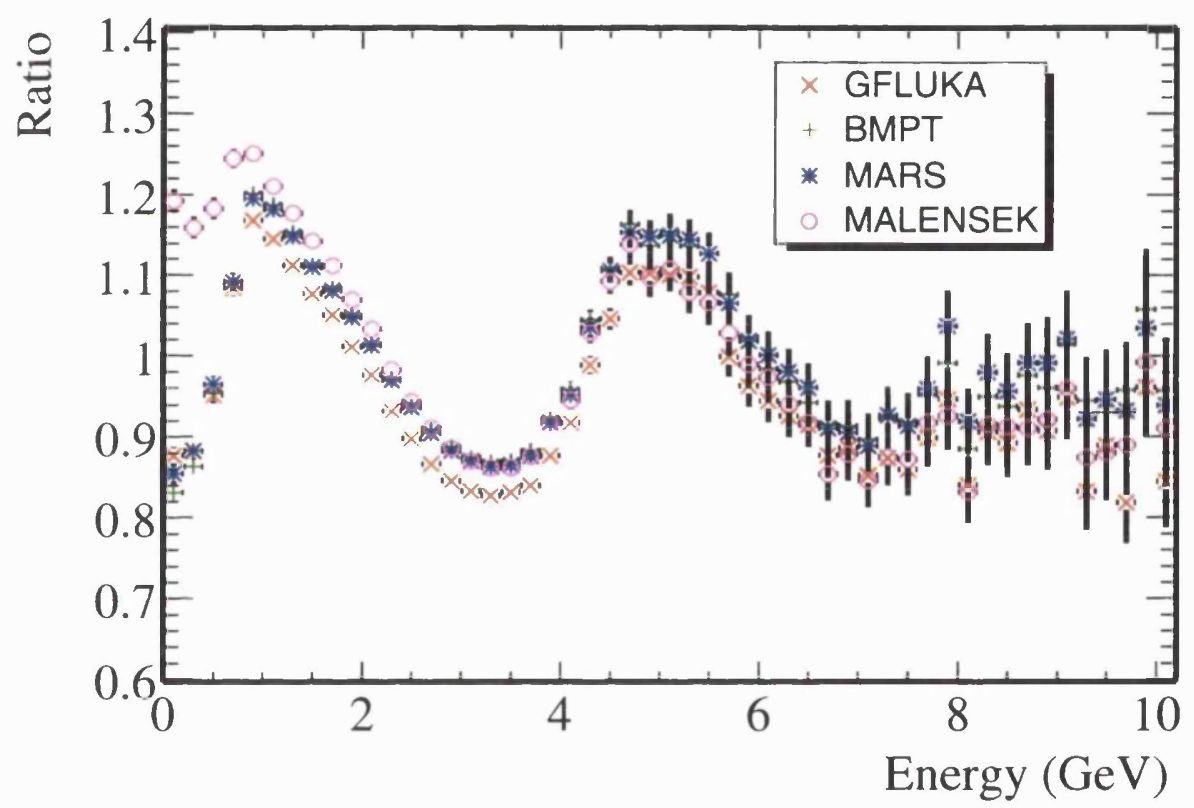

Figure 7.2: The predicted Far/Near ratio for the normalized $\nu_{\mu} \mathrm{CC}$ event rates calculated using four different hadron production models, (no oscillations). 


\subsection{GEANT simulation}

The detector geometries have been incorporated into a GEANT program in order to simulate neutrino interactions. A system has been developed to simulate the active detector response such that the output contains hits that mimic hits in the real detectors. The Fiber in Long Scintillator, (FLS) system [67], a simulation software package, takes into account such effects as: photon production in scintillator; collection efficiency of the wavelength shifting fibre as a function of particle path through scintillator strip; transmission through optical fibres and connectors; phototube response; and Front-End electronics digitizations.

A number of neutrino events $(25,000)$, approximately corresponding to 7 years of running at the Far detector with the Low Energy beam have been simulated in both Near and Far detectors. Neutrinos are randomly selected from a predicted flux to get flavour, energy, direction and position at a detector. These parameters are then used to calculate how much detector material the neutrino traverses and using a scaled cross-section (to remove factors of $\sim 10^{-9}$ ) decides whether or not the neutrino interacts. If it does interact, the interaction position and material properties at that point are passed along with the neutrino properties to NEUGEN [68] which is used to generate the event kinematics. The final state particles are then tracked by GEANT and the energy losses in the active detector are passed to the FLS system to produce detector hits.

The Low Energy neutrino beam contains mainly $\nu_{\mu}, \bar{\nu}_{\mu}$ with a small fraction of $\nu_{e}, \bar{\nu}_{e}(\sim 1 \%)$. The $\nu_{\mu}$ energy distributions shown in Section 7.1 for the four hadron production models, (and a similar set for the $\bar{\nu}_{\mu}$ ), are used to reweight the $\nu_{\mu}, \bar{\nu}_{\mu}$ CC events in both detectors. The resulting muon energy spectra for the $\sim 18500 \nu_{\mu}, \bar{\nu}_{\mu}$ CC events in the Near and Far samples, 
reweighted by the four models are shown in Figure 7.3.

To see the effects of neutrino oscillations on the Far muon energy spectra and on the Near/Far muon energy ratio, the Far detector $\nu_{\mu}$ CC events are further reweighted using the two flavour mixing oscillation probability derived in Chapter 2. Recent values from Super-Kamiokande for the atmospheric neutrino oscillation parameters of $\sin ^{2} 2 \theta=1$ and $\Delta m^{2}=0.0025 \mathrm{eV}^{2}$ are used to illustrate the oscillation signal. Figure 7.4 shows the Far muon energy spectrum with and without oscillations for the GFLUKA hadron production model. The oscillation signature is observed as a depletion of events at energies below $\sim 5 \mathrm{GeV}$. Figure 7.5 shows the normalized muon energy spectra ratios: Far/Near, Oscillated Far/Far and Oscillated Far/Near for the GFLUKA model. MINOS will therefore measure Oscillated Far/Near and compare it to Far/Near from Monte Carlo to get Oscillated Far/Far. The sensitivity of this final ratio to the oscillation parameters determines the usefulness of this measurement. 


\section{Muon Energy Spectrum - Near Detector}

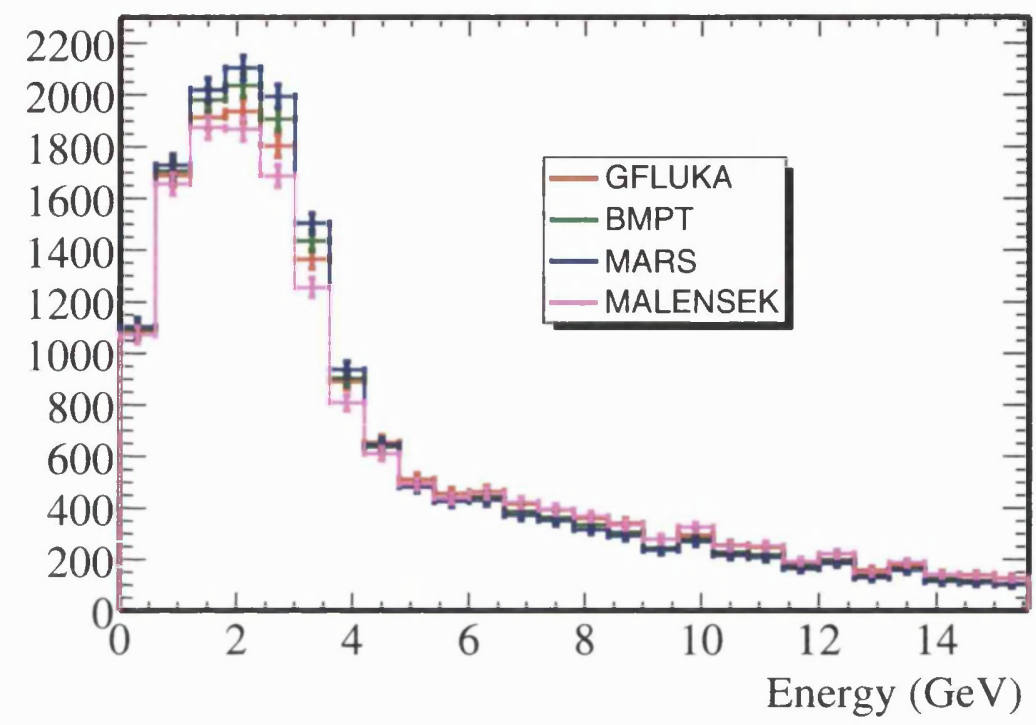

Muon Energy Spectrum - Far Detector

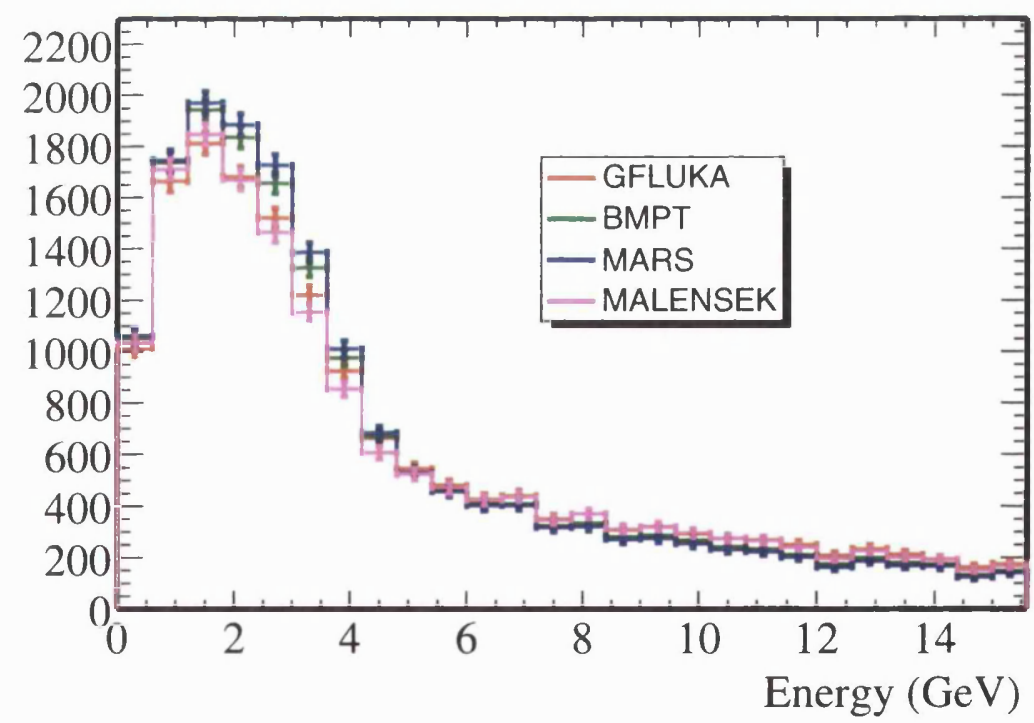

Figure 7.3: The predicted Near (upper) and Far muon energy spectra from $\nu_{\mu}$, $\bar{\nu}_{\mu} \mathrm{CC}$ interactions calculated using four different hadron production models, (no oscillations). The entire Monte Carlo event sample has been used to make these plots corresponding to $\sim 7$ years of data taking at the Far Detector. 


\section{Muon Energy Spectrum - Far Detector}

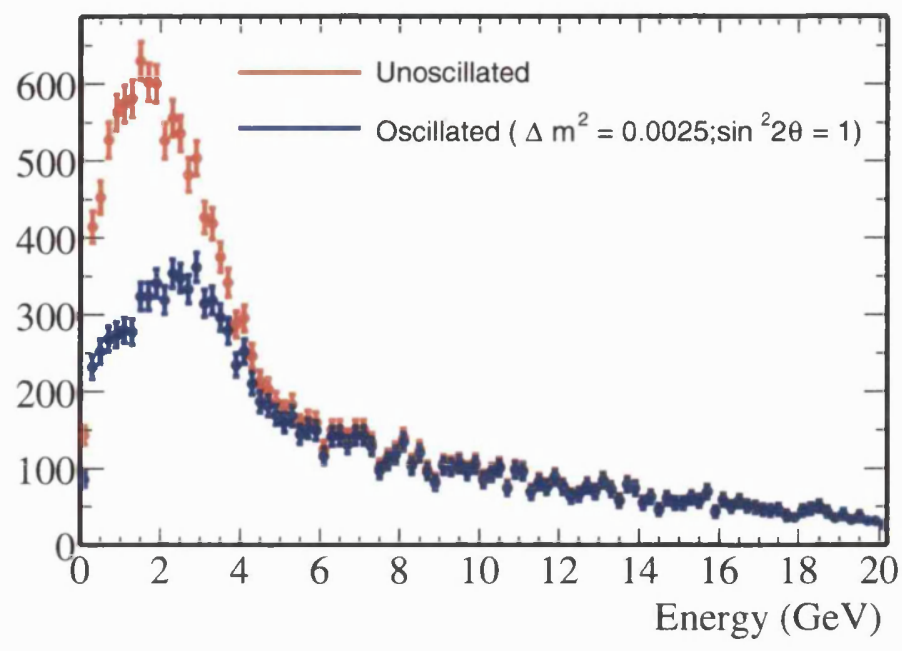

Figure 7.4: The predicted muon energy spectrum from $\nu_{\mu}, \bar{\nu}_{\mu} \mathrm{CC}$ interactions with and without oscillations using the GFLUKA hadron production model in the Far Detector. The oscillation parameters used are $\Delta m^{2}=0.0025 \mathrm{eV}^{2}$ and $\sin ^{2} 2 \theta=1$ which results in a depletion of events below $\sim 5 \mathrm{GeV}$.

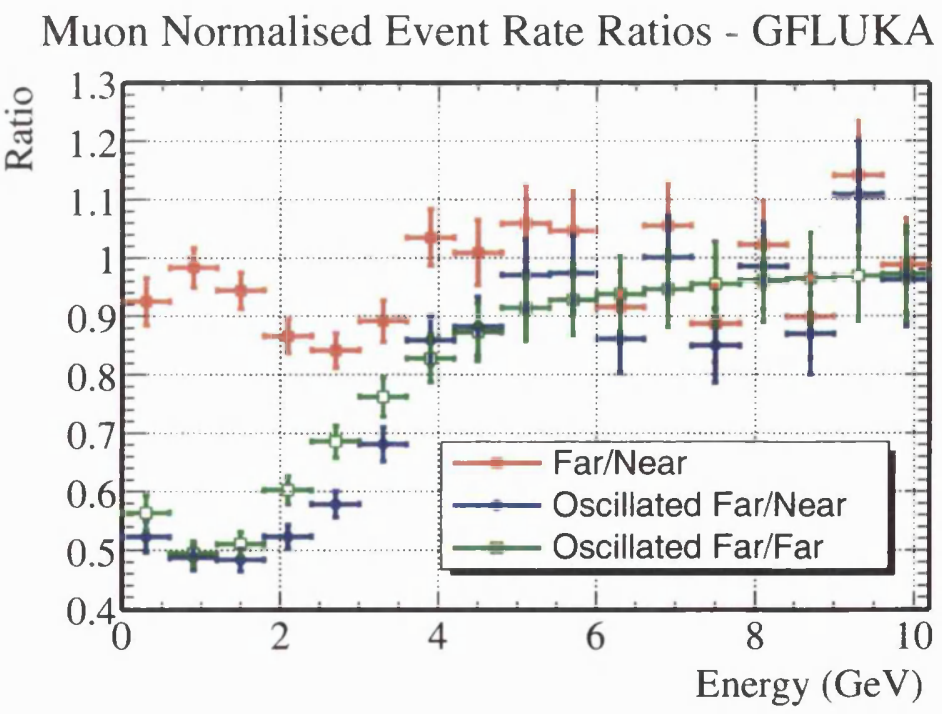

Figure 7.5: The normalized muon event rate ratios for the GFLUKA hadron production model, showing Far/Near, Oscillated Far/Far and Oscillated Far/Near. 


\subsection{Muon Reconstruction}

To properly study the measurement potential, detector effects must also be included in the muon energy spectra. For measurements of muon energy from range or curvature, only event topology is important for energy reconstruction. MINOS is able to reconstruct stopping muons with $\sim 6 \%$ energy resolution and through-going muons from curvature with $\sim 15 \%$ resolution [50].

Using the event sample obtained from the GEANT simulation, the true muon energies are smeared by randomly sampling from a Gaussian distribution with an appropriate width. A stopping muon event is defined as one in which there are no hits within $50 \mathrm{cms}$ of an edge of the detector. All other muon momenta are assumed to be reconstructed from curvature. A cut on the minimum muon energy of $0.5 \mathrm{GeV}$ is used, which reflects the fact that at lower energies, the ability to accurately reconstruct muons diminishes. Also, at lower energies there is a background from $\mathrm{NC}$ events that would be present in the real experiment but which have not been simulated in this analysis. Introducing this minimum muon energy cut minimizes the effects of this background, since above this energy the muon track is clearly identifiable in the detector. In practice, this cut need not introduce a dependence on the energy calibration of the detector, since it can be imposed by demanding a muon track length longer than $\sim 12$ planes $(\approx 30 \mathrm{~cm}$ of iron) in the detector.

Since the energy resolution for stopping muon reconstruction is significantly better than for through-going muons, it is interesting to consider the effect of only using this sub-sample to make the measurement. In the Far Detector event sample, of the $18546 \nu_{\mu}, \bar{\nu}_{\mu}$ CC events, 9823 of the muons stopped, $(\sim 53 \%)$. 


\subsection{Oscillation Parameter Measurement}

A MINOS measurement using the proposed method will compare the spectrum measured at the Far Detector with that expected from the measurements at the Near Detector. However, the Near Detector will measure many neutrino events and the statistical errors will come solely from the Far Detector. For this reason, the Near Detector muon energy spectrum is not used in this analysis, (the available Near Detector event sample is the same size as for the Far Detector and so would result in comparable errors for the two spectra). Instead, only the Far Detector spectrum is used and it is assumed that measurements of the neutrino energy spectrum at the Near Detector can precisely tune the Monte Carlo simulation of the beam.

To study the accuracy with which the oscillation parameters can be extracted using only muons from CC interactions, the Far Detector sample was split into two sub-samples. A smaller sample of approximately 7000 of the original 25,000 neutrino events is reweighted to represent an example data set for 2 years of running for each of the different models. The remaining events are used as the MC sample.

The data sample is reweighted according to one of the hadron production models, reconstructed and "oscillated" using $\sin ^{2} 2 \theta=1$ and $\Delta m^{2}=$ $0.0025 \mathrm{eV}^{2}$ to form a "measured" muon energy spectrum. The MC sample is then reweighted and reconstructed in the same fashion, but oscillated using many different combinations of $\sin ^{2} 2 \theta$ and $\Delta m^{2}$. The data and the MC spectra are then normalized such that the area under each spectrum is 1 and the shapes of the spectra are compared. In doing this, the absolute number of events measured, which potentially contains important information about the occurance of oscillations, is not being compared to that expected from Monte Carlo. Clearly using this extra information can give a more sensi- 
tive measurement, however it is also dependent on accurate prediction of the number of events expected. For this reason, a procedure looking only at the spectral shape is valuable. For each combination of $\mathrm{MC}$ oscillation parameters a $\chi^{2}$ value, representing differences in the spectral shapes, is calculated using:

$$
\chi^{2}=\sum_{i=1}^{i=m a x} \frac{\left(D A T A_{i}-M C_{i}\right)^{2}}{\left(\sigma_{D A T A_{i}}^{2}+\sigma_{M C_{i}}^{2}\right)}
$$

where $i$ represents the energy bins in the spectra and max is the maximum energy bin used in the calculation; DAT $A_{i}, \sigma_{D A T A_{i}}^{2}, M C_{i}, \sigma_{M C_{i}}^{2}$ are the number of events and error on the number of events in bin $i$ for the data and MC sample respectively. In this analysis the $\chi^{2}$ has been calculated from 0 out to $20 \mathrm{GeV}$, beyond which the statistics become low and the oscillation effects small.

The result of this procedure is a two-dimensional histogram of $\Delta m^{2}$ versus $\sin ^{2} 2 \theta$ weighted by $\chi^{2}$. An example of this is shown in Figure 7.6, created using all CC muons and the GFLUKA model. Also shown is the $\chi^{2}$ distribution for all the data/MC spectra comparisons. The $\chi^{2}$ in this case was calculated using 100 bins, however normalizing the spectra reduces the number of degrees of freedom by 1 . The observed peak in the distribution agrees well with the expected value of 99 , indicating that the fitting method is valid.

Figure 7.7 shows the normalized muon energy spectrum for the data, the $\mathrm{MC}$ best fit and the unoscillated MC spectrum for comparison.

Figure 7.8 shows $\Delta \chi^{2}$ contours from the minimum representing the $68.3 \%$, $90.0 \%$ and $95.5 \%$ confidence limits. This procedure has been carried out for each hadron production model. Figure 7.9 shows the $90 \%$ confidence level for each model. 

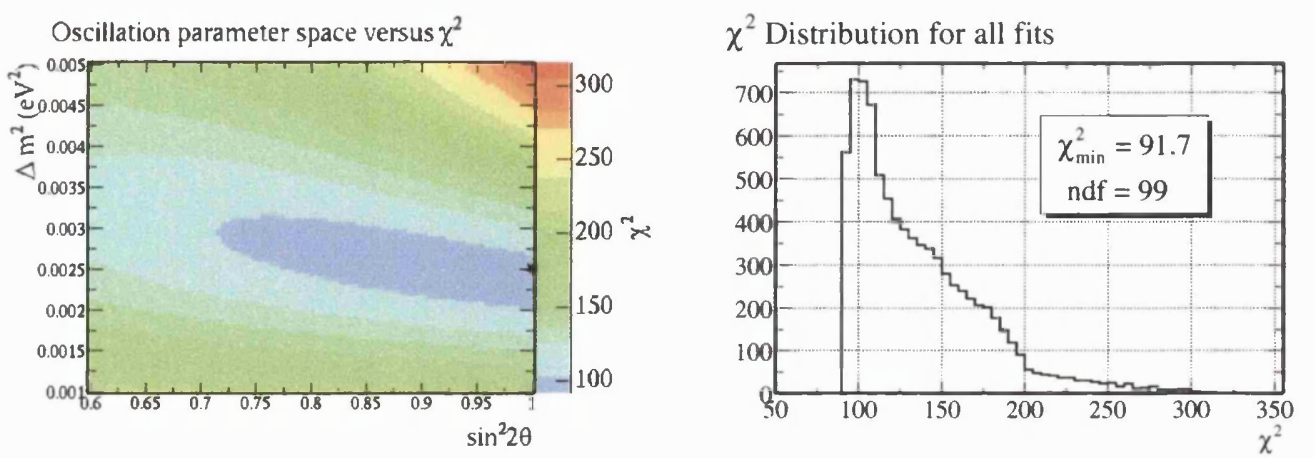

Figure 7.6: A $\chi^{2}$ fit to the data using the MC sample weighted with many different values for the oscillation parameters, (GFLUKA model). The plot on the left shows how the $\chi^{2}$ varies over the parameter space and the plot on the right is a histogram of all the $\chi^{2}$ values.

From this single measurement, at the $90 \%$ confidence level after two years running, given the current uncertainties in the neutrino energy spectrum at the Far Detector, the oscillation parameters have been measured to $\sim 26 \%$ for $\sin ^{2} 2 \theta$ and $\sim 30 \%$ for $\Delta m^{2}$. The errors quoted are found by looking for the maximum deviation of a parameter from the best fit along the contour in the physical region. The accuracy quoted above is a worst case measurement should the neutrino spectrum at the Far Detector be described by the BMPT hadron production model. In the best case, should the MALENSEK model provide the best description, $\sin ^{2} 2 \theta$ can be measured to $\sim 19 \%$ and $\Delta m^{2}$ to $\sim 23 \%$.

The variations in accuracy of the $\sin ^{2} 2 \theta$ measurement comes from the differences in predicted event rates at the lower energies. Figure 7.10 shows the differences in normalised event rates as a function of energy for each model relative to GFLUKA. This plot is made by scaling each spectrum by the amount required to normalise the GFLUKA spectrum and then subtracting the GFLUKA spectrum from each. The sizes of the contours in Figure 7.9 
Normalised Muon Energy Spectrum - Far Detector

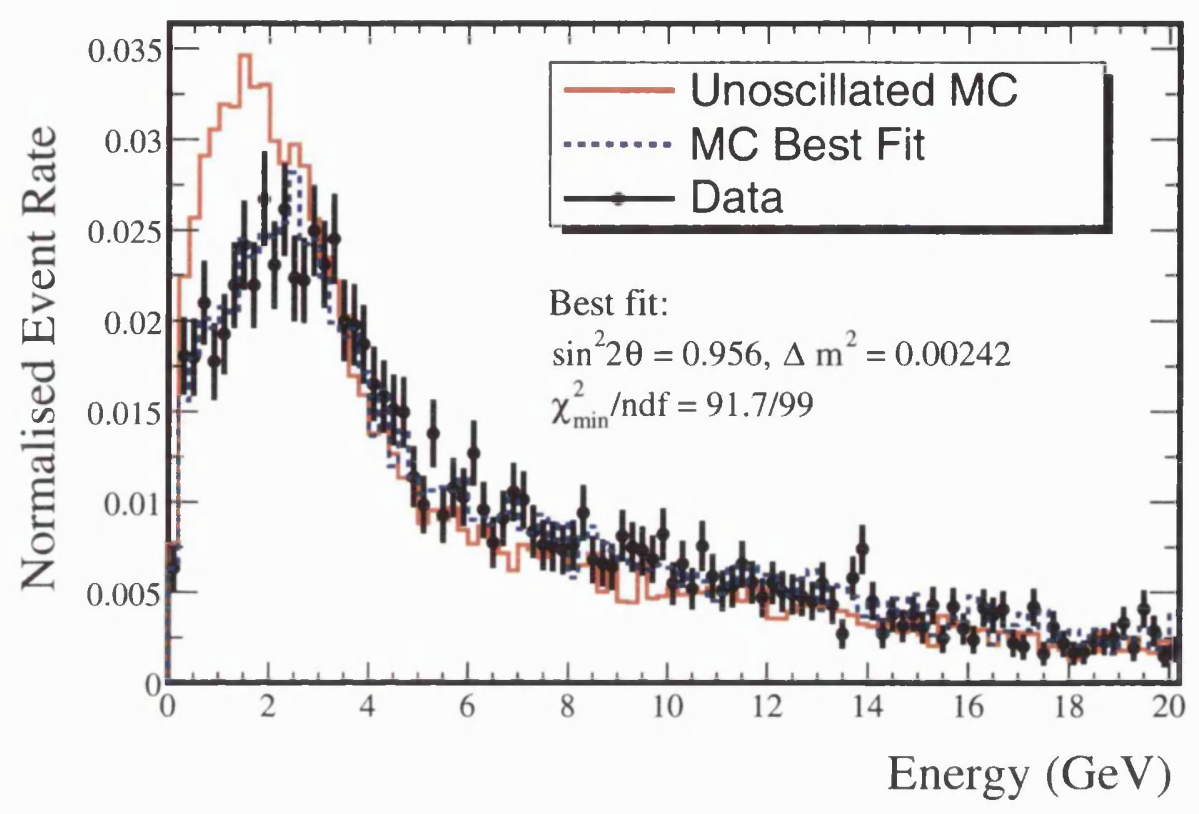

Figure 7.7: The normalized muon energy spectra for the data and the MC best fit, (using the GFLUKA hadron production model).

therefore reflect the difference in statistics at the oscillation sensitive energies for each model.

\section{Unphysical Regions}

The method for calculating confidence limits used in this analysis does not recognize the physical boundary at $\sin ^{2} 2 \theta=1$ and the $\chi^{2}$ is calculated even for unphysical sets of oscillation parameters. This was done because of the choice of the "true" value of $\sin ^{2} 2 \theta$ to be 1 . Statistical fluctuations are expected to cause the best fit value for $\sin ^{2} 2 \theta$ to be greater than 1 for some data samples, even though, clearly, a measurement of $\sin ^{2} 2 \theta>1$ has no physical interpretation. The alternative is to restrict the fit to be in the physical region, however, in doing this, it was thought that the size of the 


\section{Confidence Limits - GFLUKA}

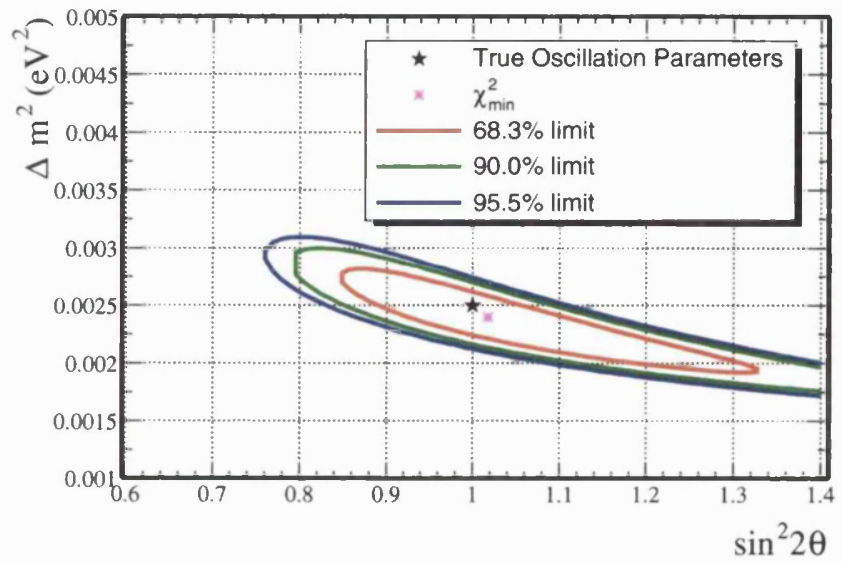

Figure 7.8: The confidence limits after 2 years data taking on the neutrino oscillation parameters measured using the reconstructed muon energy spectrum, (GFLUKA hadron production model). The $68.3 \%, 90.0 \%$ and $95.5 \%$ limits are shown.

confidence limits may be incorrectly reported.

A better method for calculating confidence limits close to a physical boundary has been described by Feldman and Cousins [69]. The approach advocates a particular ordering principle when constructing the usual Neyman confidence belts. The application of the Feldman-Cousins, $(\mathrm{F} / \mathrm{C})$ procedure to the two parameter case, such as for neutrino oscillation parameters, leads to a modification of the $\Delta \chi^{2}$ value corresponding to a particular confidence level at each point in $\sin ^{2} 2 \theta-\Delta m^{2}$ space. The deviation of the limiting $\Delta \chi^{2}$ values from standard values is largest close to the physical boundary and, away from this boundary, the $\mathrm{F} / \mathrm{C}$ values approach the standard values.

To construct the correct $\mathrm{F} / \mathrm{C}$ confidence limits, a large number of fake experiments are performed at each point in $\sin ^{2} 2 \theta-\Delta m^{2}$ space, using Monte Carlo. This is therefore a very computer intensive process and has not been carried out for this analysis. Further, a previous comparison of the $\mathrm{F} / \mathrm{C}$ and 
90\% Confidence Limits after 2 years running (all muons)

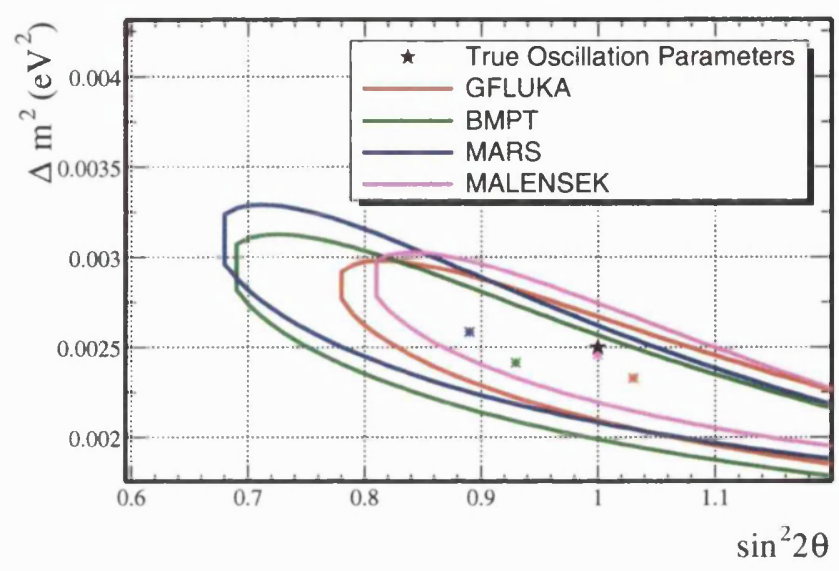

Figure 7.9: The $90 \%$ confidence limits after 2 years data taking on the neutrino oscillation parameters measured using the reconstructed muon energy spectrum for the four hadron production models.

the usual $\Delta \chi^{2}$ procedure have shown that, for a large oscillation signal in MINOS, the difference in size of the confidence limits is small [70].

When interpreting the confidence limits shown here, although the coverage in the physical region is not necessarily correct (since the best fit point may be in the unphysical region), the extent of the contours from the best fit point reflect the accuracy with which the oscillation parameters can be measured. 
Differences in Predicted $v_{\mu}$ CC Energy Spectrum - Far Detector

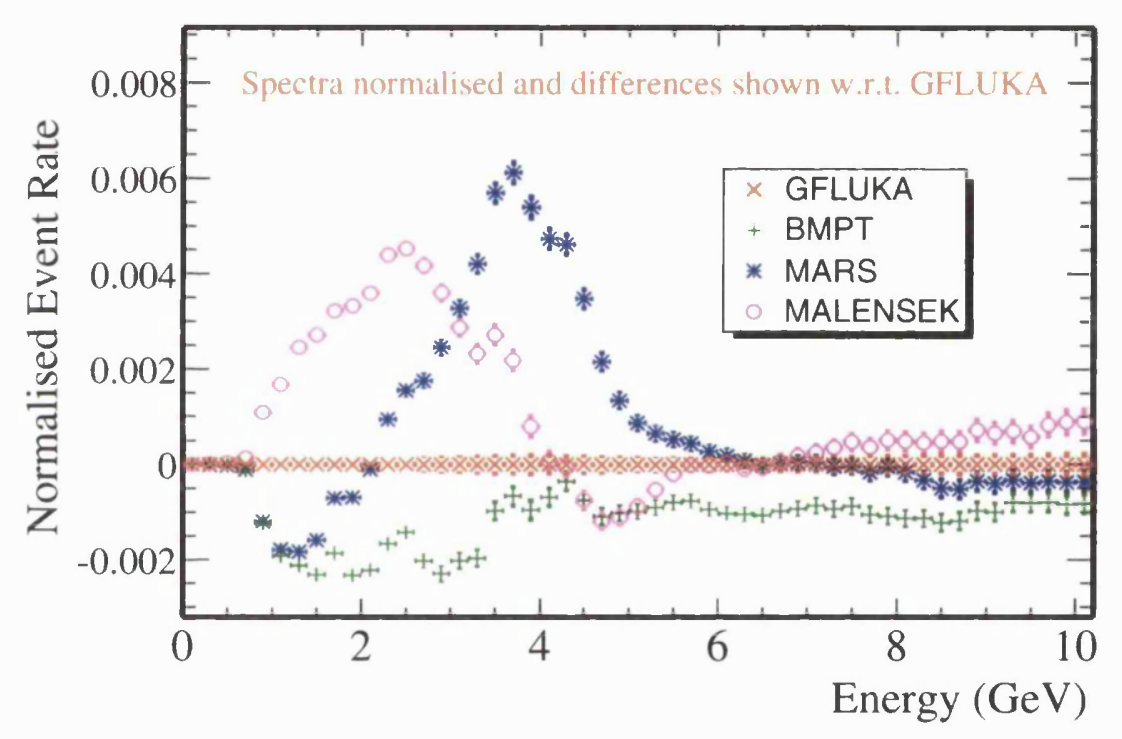

Figure 7.10: Differences in the predicted $\nu_{\mu}$ CC energy spectrum at the Far Detector between the different hadron production models (no oscillations). The differences in the normalised event rates predicted by BMPT, MARS and MALENSEK with respect to those from GFLUKA are shown. 


\subsection{Other Measurements using Muons}

Using the same MC data set, confidence limits can be drawn for different measurements. For example, rather than looking at all muon events, only contained events, which have better energy resolution, are considered. Also, a cut that rejects events with more than some limiting energy in the shower has been considered. Neutrino oscillations for the currently favoured parameters predominantly reduces the neutrino flux at energies less than 5 $\mathrm{GeV}$. Cutting above this energy in the shower removes events that do not contribute significantly to the oscillation signal.

Further, in the limit that there is no shower and the interaction is a quasielastic scatter, the muon carries all the energy of the original neutrino. This allows the neutrino energy spectrum to be measured with excellent resolution, although the accuracy of the measurement becomes limited by statistics. It is important to note that in this analysis, the cut on the shower energy has been made using the $\mathrm{MC}$ truth information. No attempt is made to account for the issues involved in reconstructing showers in real data. Also, it is clear that in real data, this cut re-introduces a dependency on the energy calibration of the detectors.

Figure 7.11 shows the fraction of events accepted for a range of shower energy cuts and Figure 7.12 shows the $90 \%$ confidence limits for the GFLUKA model when using different values for the maximum shower energy.

Considering Figures 7.11 and 7.12, a cut on the shower energy of $1 \mathrm{GeV}$ optimizes the accuracy of the measurement. To see why this may be, Figure 7.13 shows the unoscillated muon energy spectra in the Far Detector for a range of different cuts on the shower energy. The entire sample of Monte Carlo events was used to make this plot thus the total number of events plotted corresponds to approximately 7 years' data. It can be seen that the 


\section{Fraction of Events Passing Shower Energy Cut}

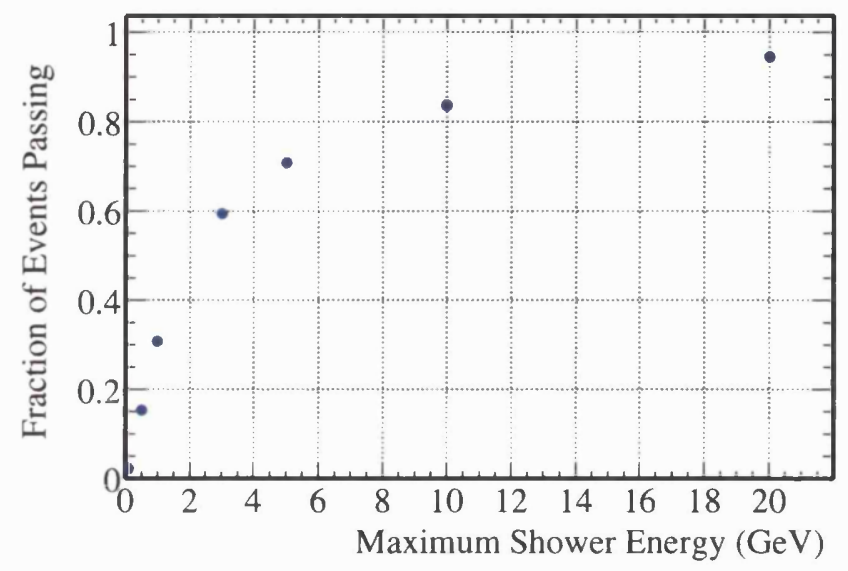

Figure 7.11: The fraction of neutrino events accepted for a range of maximum shower energies.

relative proportion of events in the oscillation sensitive region increases as the cut energy gets lower. Moreover, for the lower shower energies, events in this region are constrained to correspond to neutrinos of a similar energy. Therefore, above $1 \mathrm{GeV}$, the resolution of the oscillation signal is diminished, and below this the statistical errors begin to dominate. For the $1 \mathrm{GeV}$ case, the $90 \%$ contour indicates a 8-9\% measurements for both $\sin ^{2} 2 \theta$ and $\Delta m^{2}$.

It is interesting to compare the parameter contours for the muon measurements with a similar contour for a measurement of the oscillation parameters using the reconstructed neutrino energy spectrum. To reconstruct the neutrino energy, the reconstructed muon energy is found in the same way as before, and the reconstructed shower energy is found by smearing the true energy in the shower by $55 \% / \sqrt{E_{s h w}}$. Figure 7.14 shows three $90 \%$ confidence limits: reconstructed muon energy, reconstructed neutrino energy and reconstructed muon energy for events with less than $1 \mathrm{GeV}$ in the shower.

The neutrino contour indicates a parameter measurement of $\sim 12 \%$ on 
90\% Confidence Limits after 2 years running (GFLUKA)

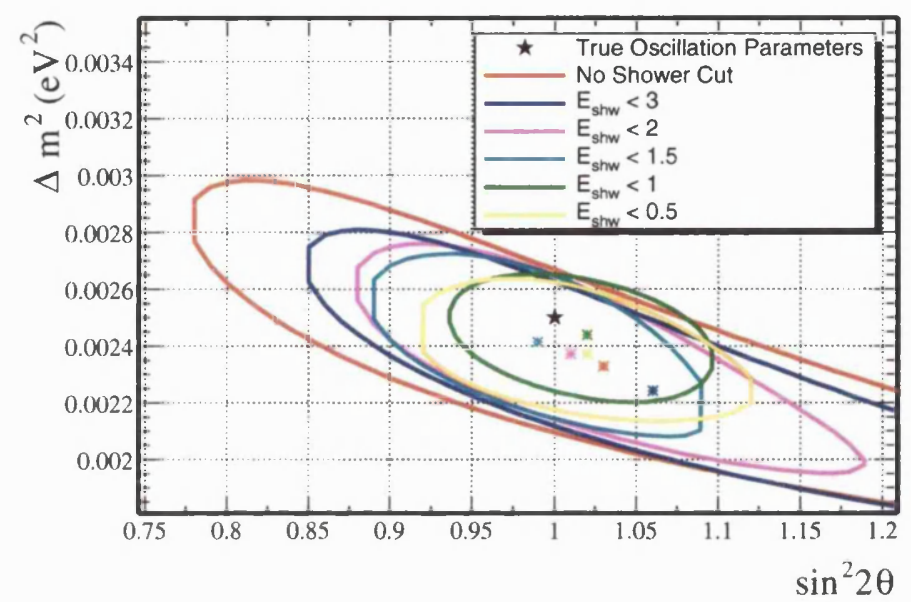

Figure 7.12: The $90 \%$ confidence limits after 2 years data taking on the neutrino oscillation parameters measured using the reconstructed muon energy spectrum for a range of cuts on the shower energy in the event. The contour becomes smaller as the limiting energy is reduced to $1 \mathrm{GeV}$, after which the contour begins to expand again because of the decreasing statistics.

$\sin ^{2} 2 \theta$ and $\sim 15 \%$ on $\Delta m^{2}$, larger than using the muon spectrum. With the muon measurement, unlike in the case of the neutrino measurement, there is an extra dependency on the Monte Carlo. The $y$-distribution, which gives the fraction of the neutrino energy which goes into the hadron shower, must be included in the Monte Carlo in order to extract the oscillation parameters. Any uncertainties in this distribution would lead to systematic errors in the muon measurement. In particular, any differences in the number of events passing the shower cut will change the statistical significance of the measurement.

The results presented here assume perfect knowledge of the $y$-distribution. At the MINOS Near detector, there are many neutrino events every year, and the $y$-distribution can be well measured at higher energies. At lower 


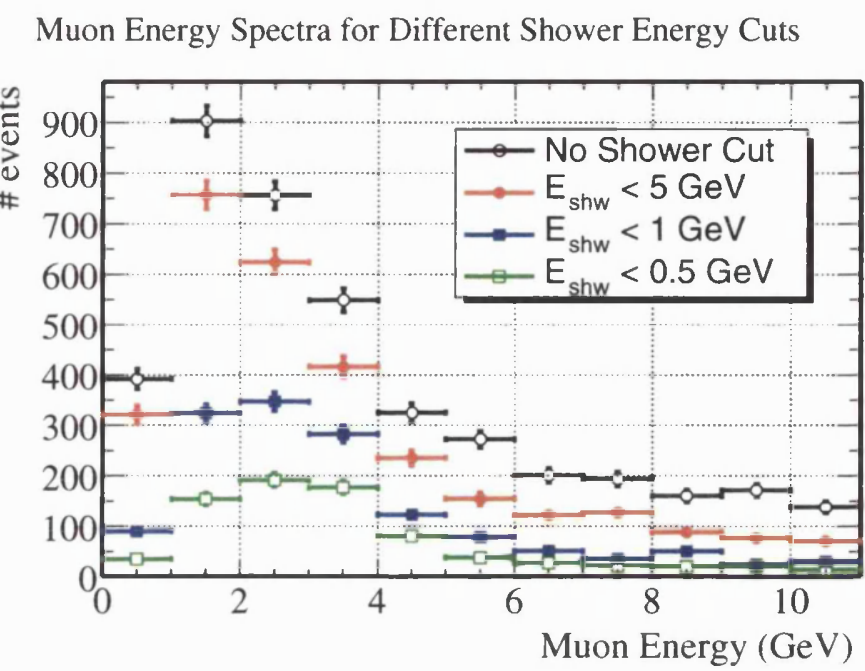

Figure 7.13: Muon energy spectra in the Far Detector without oscillations for a range of cuts on the maximum shower energy in an event. The spectra correspond to approximately 7 years worth of data.

energies, muon momentum measurements will introduce systematic errors in the $y$-distribution at the level of $5 \%$ [71]. To understand the effects of any systematic errors, a simple approach is to use the true $y$ values from the data event sample and introduce a systematic shift. At lower energies, the mean $y$ value is $\sim 0.4$. A $5 \%$ systematic shift can therefore be approximated by adding \pm 0.02 to all $y$ values in the data sample. Figure 7.15 shows the contours for $\pm 5 \%$ systematic shifts in the $y$-distribution.

If the mean $y$ value in the current Monte Carlo have been overestimated by $5 \%$ compared to the data, the $-5 \%$ systematic shift contour is obtained. This contour is slightly smaller than before, reflecting the increased number of events passing the $1 \mathrm{GeV}$ shower cut, and the best fit point is shifted by $\sim 2 \%$ in $\sin ^{2} 2 \theta$ and $\sim 4 \%$ in $\Delta m^{2}$. If the Monte Carlo $y$ value has been underestimated compared to the data, then the $+5 \%$ systematic shift contour shows that there are less events passing the shower cut and measurement 
90\% Confidence Limits after 2 years running (GFLUKA)

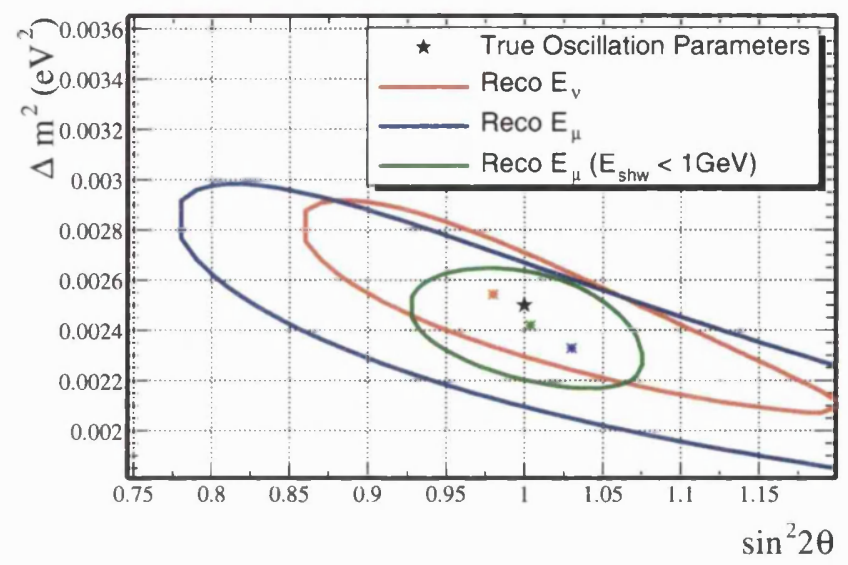

Figure 7.14: The $90 \%$ confidence limits after 2 years data taking on the oscillation parameters measured using the muon and neutrino energy spectra.

sensitivity is reduced. In this case, the best fit point is shifted by $\sim 2 \%$ in $\sin ^{2} 2 \theta$ and $\sim 10 \%$ in $\Delta m^{2}$.

Table 7.1 shows the percentage errors in the physical region for two of the cuts described above for each of the hadron production models: looking both at contained muon events, and also all muon events in which there is less than $1 \mathrm{GeV}$ in the shower. As would be expected from the reduced statistics, the errors on the measurement when just using contained events are larger than for using all muons. The cut on the shower energy significantly improves the measurement for all models despite the lower statistics. The best measurement of the oscillation parameters using muons would therefore come from using the quasi-elastic sample. From the largest $90 \%$ contour of the four hadron production models, this gives an error of $7.6 \%$ on $\sin ^{2} 2 \theta$ and $9.6 \%$ on $\Delta m^{2}$, where the errors quoted represent the largest deviation from the best fit point along the contour in the physical region. These measurements assume perfect knowledge of the y-distribution. 
90\% Confidence Limits after 2 years running (GFLUKA)

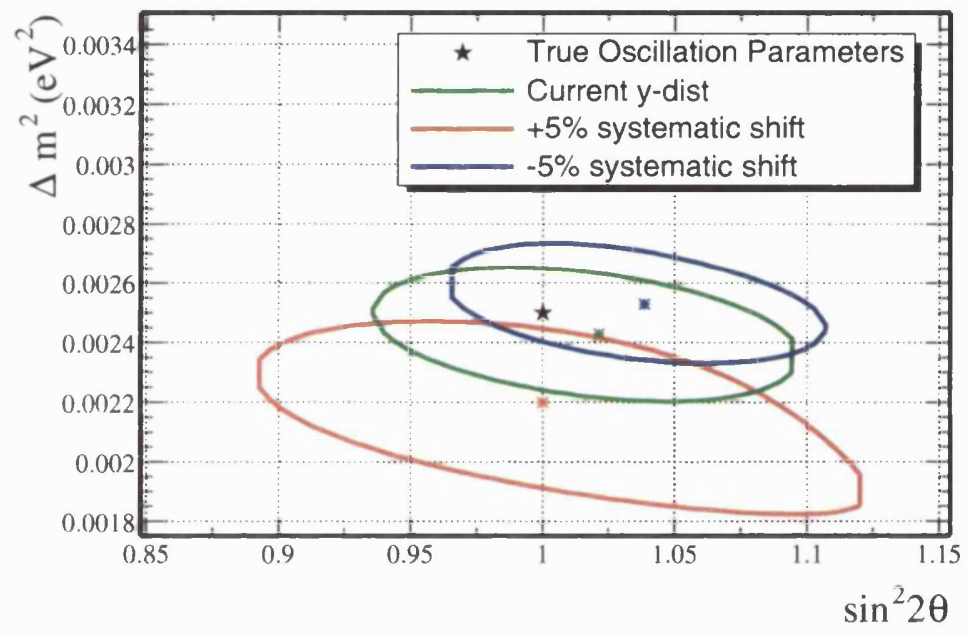

Figure 7.15: The $90 \%$ confidence limits after 2 years data taking on the oscillation parameters measured using the muon energy spectrum from quasi-elastic CC events showing the effect of systematic errors in the $y$-distribution. The red $+5 \%$ systematic shift refers to a $5 \%$ increase in $y$ for the data sample, that is, the Monte Carlo underestimates $y$. Similarly, for the blue $-5 \%$ systematic shift. 


\begin{tabular}{|c||c||c|c|c||}
\hline \multirow{2}{*}{ Model } & \multirow{2}{*}{ Parameter } & \multicolumn{3}{c||}{ Muon Sample } \\
\cline { 3 - 5 } & & All Muons & Contained & $E_{\text {shw }}<1 \mathrm{GeV}$ \\
\hline \hline \multirow{2}{*}{ GFLUKA } & $\sin ^{2} 2 \theta$ & 24.3 & 27.4 & 8.4 \\
\cline { 2 - 5 } & $\Delta m^{2}$ & 28.1 & 36.3 & 9.3 \\
\hline \multirow{2}{*}{ BMPT } & $\sin ^{2} 2 \theta$ & 25.8 & 27.3 & 8.3 \\
\cline { 2 - 5 } & $\Delta m^{2}$ & 29.6 & 37.0 & 9.4 \\
\hline \multirow{2}{*}{ MARS } & $\sin ^{2} 2 \theta$ & 23.6 & 30.3 & 8.3 \\
\cline { 2 - 5 } & $\Delta m^{2}$ & 27.3 & 39.8 & 9.3 \\
\hline \multirow{2}{*}{ MALENSEK } & $\sin ^{2} 2 \theta$ & 19.0 & 22.2 & 7.6 \\
\cline { 2 - 5 } & $\Delta m^{2}$ & 23.2 & 29.3 & 8.6 \\
\hline
\end{tabular}

Table 7.1: Percentage errors on oscillation parameter measurements for different hadron production models for 2 years of data. Three different measurements are shown: using all muons from CC interactions, using only contained muons and using all muons for which the shower energy in the event is less than $1 \mathrm{GeV}$. 


\subsection{Beam Misidentification}

In the previous section, it was assumed that having measured the Near Detector energy spectrum, the Monte Carlo simulation can be tuned and the Far Detector spectrum predicted. In this section, the consequences of incorrectly tuning the Monte Carlo are considered using the four hadron production models. The data sample is now reweighted as before using one model, however the MC sample is reweighted using a different model in order to investigate the effect on the oscillation parameter measurement.

For the study to be meaningful, the Near Detector spectrum must now also be included, as the relevant measurable quantity is the Far/Near ratio. Since the statistical accuracy of the measurement is dominated by the Far Detector spectrum, the entire Near Detector sample (of approximately 18,500 $\nu_{\mu}, \bar{\nu}_{\mu}$ CC events) is used to calculate the ratio for both the data and MC sample. The Near Detector events are reweighted according to one of the hadron production models and reconstructed as for the Far Detector.

To perform this test, the quasi-elastic sample as described in the previous section is used. Figure 7.16 shows four plots, each of which employs a different hadron production model to generate the data sample. In each plot there are four contours representing the $90 \%$ confidence limits when the four different hadron production models are used for the MC sample.

The relative sizes of the contours for each data sample correlates with the statistical errors expected from Figure 7.10 as before. There are only small differences of $\sim 1-2 \%$ in both $\sin ^{2} 2 \theta$ and $\Delta m^{2}$ at the best fit points in each case. After 2 years running, the errors on the measurements of $\sin ^{2} 2 \theta$ and $\Delta m^{2}$ are therefore dominated by statistics and the current systematic uncertainties in the hadron production model at the target are a comparatively small contribution. 

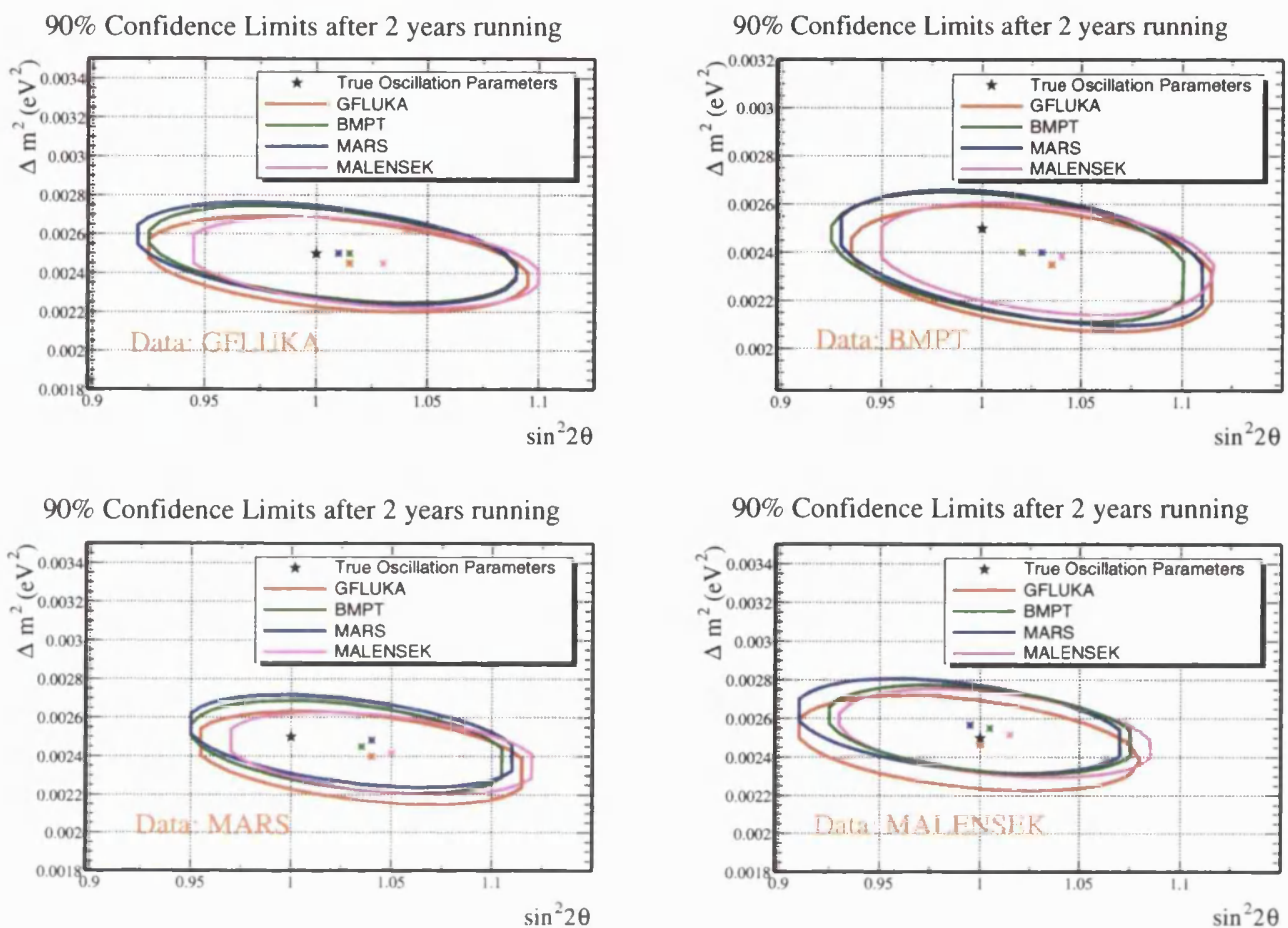

Figure 7.16: $90 \%$ confidence limits showing the effect of misidentifying the hadron production model. The four plots each use a different hadron production model to give the Far/Near ratio for the data sample. The contours in each plot then give the $90 \%$ confidence limits when using each of the different hadron production models to give the Far/Near ratio for the MC sample.

\subsection{Summary}

In this section, a neutrino oscillation parameter measurement using muons from $\nu_{\mu}, \bar{\nu}_{\mu}$ CC interactions for $\sin ^{2} 2 \theta=1$ and $\Delta m^{2}=0.0025 \mathrm{eV}^{2}$ has been described. The advantage of this measurement is that it is independent of the energy calibration of the detectors. After two years, a measurement of $\sin ^{2} 2 \theta$ to $\sim 26 \%$ and $\Delta m^{2}$ to $\sim 30 \%$ could be made using neutrinos from the Low Energy beam.

The sensitivity of the muon energy spectrum to oscillations is limited 
because the muon energy does not directly correlate with the energy of the original neutrino. Rather, the energy of the muon in a CC interaction is given by the $y$-distribution, which describes the fraction of the original neutrino energy in the shower. Cutting on this shower energy at $1 \mathrm{GeV}$ produces a sample of quasi-elastic events in which the energy of the muon is much more correlated with the energy of the original neutrino. However, the dependence on the energy calibration of the detector is re-introduced both in the shower energy cut, and in any measurement of the $y$-distribution using the Near Detector. Using this sample a measurement of the oscillation parameters has been made to $7.6 \%$ for $\sin ^{2} 2 \theta$ and $9.6 \%$ for $\Delta m^{2}$, assuming perfect knowledge of the $y$-distribution.

It has been shown that $5 \%$ systematic errors in the $y$-distribution have significant effects on this measurement: $2 \%$ shifts in $\sin ^{2} 2 \theta$ and up to $10 \%$ shifts in $\Delta m^{2}$ at the best fit points. Further, a miscalibration of the absolute energy scale would lead both to a difference in the number of events passing the cut, and therefore the statistical accuracy of the measurement, and also to a systematic error on the position of the best fit points. This dependence on the calibration would produce identical effects to those arising from a systematic error in the $y$-distribution.

Finally, the consequences of imperfect modeling of the neutrino beam have been estimated. For the four models used in this analysis, the systematic uncertainties in the Far/Near ratio are small compared to statistical errors after two years running. 


\section{Chapter 8}

\section{Conclusions}

The MINOS detectors have been designed to achieve a relative calibration to $2 \%$ and an absolute calibration to $5 \%$. Tools for carrying out the calibration procedure have been developed and used on data from the Calibration Detector at CERN. A relative calibration to $2 \%$ has been demonstrated using the MINOS Light Injection system and cosmic ray muons to correct muon and electron event samples. A method for setting the scale for the absolute calibration has also been outlined by using muons from the test-beam.

Using cosmic ray muons, the stability of the system has been investigated over a two week period and shown to be stable to $\sim 2 \%$. Changes in the light output have been shown to correlate well with temperature changes in the vicinity of the detector.

A Monte Carlo study has been carried out to investigate the potential of measuring neutrino oscillation parameters using only the muon energy spectrum from charge current $\nu_{\mu}, \bar{\nu}_{\mu}$ interactions and the Low Energy beam. Different hadron production models have also been compared.

Assuming two-flavour mixing with $\Delta m^{2}=0.0025 \mathrm{eV}^{2}$ and $\sin ^{2} 2 \theta=1 \mathrm{a}$ shape analysis of the Far Detector spectrum has been carried out. Using all 
muons, a calibration free parameter measurement of $\sin ^{2} 2 \theta$ to $\sim 26 \%$ and $\Delta m^{2}$ to $\sim 30 \%$ with two years worth of data has been made.

Using only muons from quasi-elastic interactions, a measurement of $\sin ^{2} 2 \theta$ to $7.6 \%$ and $\Delta m^{2}$ to $9.6 \%$ has been made with two years worth of data, given current knowledge of low energy neutrino interaction kinematics. This measurement is not calibration free and so highlights the importance of the CalDet measurements for making accurate measurements of the oscillation parameters.

The effect of incorrectly modelling the neutrino beam has also been investigated using the muon measurement. With two years worth of data, the systematic uncertainties from the different models are small compared to the statistical errors on the measurement.

In contrast to atmospheric neutrino experiments, the use of a beam and a near detector removes many of the uncertainties associated with the measurement of an oscillation signal. For this reason, a measurement made with MINOS in 2007, after 2 years of data taking, will provide conclusive evidence as to whether $\nu_{\mu}$ oscillations are the cause of the atmospheric neutrino anomaly.

The K2K experiment, also a long baseline oscillation experiment, has already produced results similar to those expected from MINOS. They have observed fewer events at Super-Kamiokande than expected from measurements of the neutrino flux at their near detector and the probability that their results can be explained by a statistical fluctuation from the no oscillations hypothesis is less than $1 \%$ [72]. K2K will improve their statistics over the coming years and may confirm the oscillation signal reported by Super-Kamiokande. However, the current oscillation parameters suggested by Super-Kamiokande are at the limits of the K2K sensitivity and any mea- 
surement will be limited by statistics.

A measurement with MINOS of the type described in Chapter 7 would produce a parameter measurement of better than $10 \%$ in both $\sin ^{2} 2 \theta$ and $\Delta m^{2}$. This measurement in 2007 would significantly improve the limits provided by Super-Kamiokande and, having more statistics, would produce a more accurate measurement of the oscillation parameters than the $\mathrm{K} 2 \mathrm{~K}$ experiment. 


\section{Appendix A}

\section{A Function to describe the Observed Energy Loss by \\ Muons in Scintillator}

\section{A.1 Muon Energy Spectra}

Energy loss in thin strips of material is well described by a Landau distribution. The distribution is characterized by having a narrow peak and a long tail out to high energies. Muons passing through MINOS scintillator will deposit energy according to a Landau distribution, however, after photon capture by the wavelength shifting fibre and attenuation of the light in the fibre, the energy distribution at the phototube will be broader. Photons arriving at the phototube as a result of the passage of a muon are subject to Poisson statistics, and the total number of photoelectrons at the photocathode will depend on the typical light level produced by the particle in the scintillator. The final distribution in terms of ADCs (or some unit reflecting the total amount of charge after photomultiplication) for many muon cross- 
ings is much broader than the original Landau, however the effect of the long tail out to high energies is still visible.

An example of a typical muon energy spectrum measured at the Calibration Detector (CalDet) is shown in Figure A.1. Important features of the spectrum include the single photoelectron peak visible at $~ 80$ ADC counts; the trailing edge of the pedestal at $\sim 20$ ADC counts (the sharp cut-off is due to sparsification of the data) and the long tail out to over 1000 ADC counts (over $\sim 12$ photoelectrons).

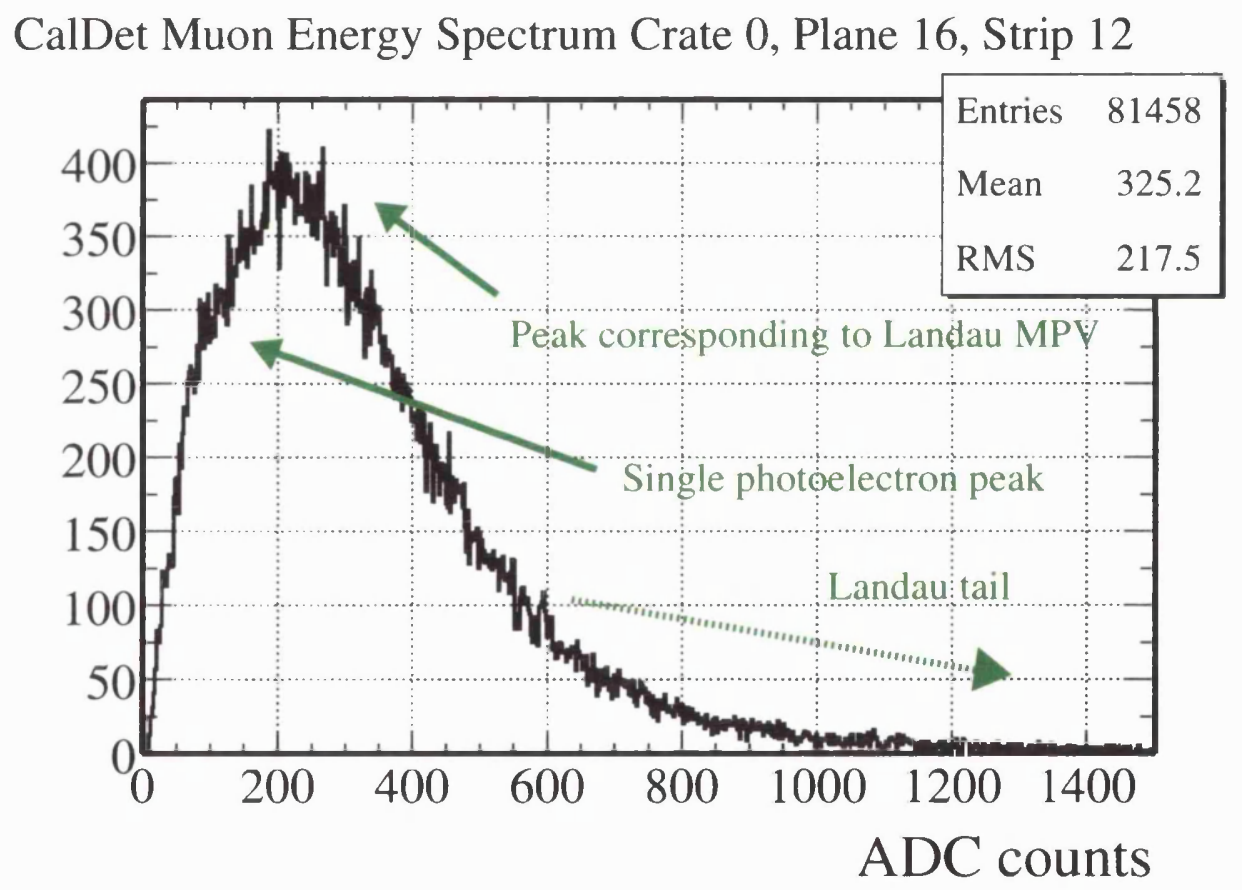

Figure A.1: A muon energy spectrum measured at the Calibration Detector. The plot is annotated showing the more distinct features of the spectrum that need to be modeled. 


\section{A.2 Simulations}

In order to develop a function to describe the muon energy spectra, a simulation of the Hamamatsu phototube using the MINOS dynode resistor chain was used to generate a typical CalDet spectrum with many entries. The advantage of doing this, is that the position and widths of the photoelectron peaks are exactly known in the simulation.

Parameters for the Landau distribution were chosen based on energy depositions in scintillator observed in GEANT simulations of the CalDet. An example distribution for $3.5 \mathrm{GeV}$ muons is shown in Figure A.2. Values randomly selected from the Landau distribution were then converted into photoelectrons based on the light levels observed at CalDet. The expected broadening due to, for example, attenuation in the fibres, was not accounted for since the dominant width in the final spectra is associated with the phototube.

A generated spectrum with 100,000 entries is shown in Figure A.3. The features observed in the CalDet spectrum are replicated in the simulation. This spectrum was then used to develop the function.

\section{A.3 The Function}

By convolving a Landau with a Poisson distribution, the initial photoelectron spectrum at the photocathode is obtained. This function alone has been used to fit muon energy spectra in the CalDet (see Section 6.2.5) with some success. However, doing this requires that the width of the underlying Landau is huge to account for the phototube width. For a more realistic description, the question is how to go from the initial photoelectron spectrum to the ADC distribution. 
Simulated Energy Loss Distribution for 3.5 GeV Muons in MINOS Scintillator

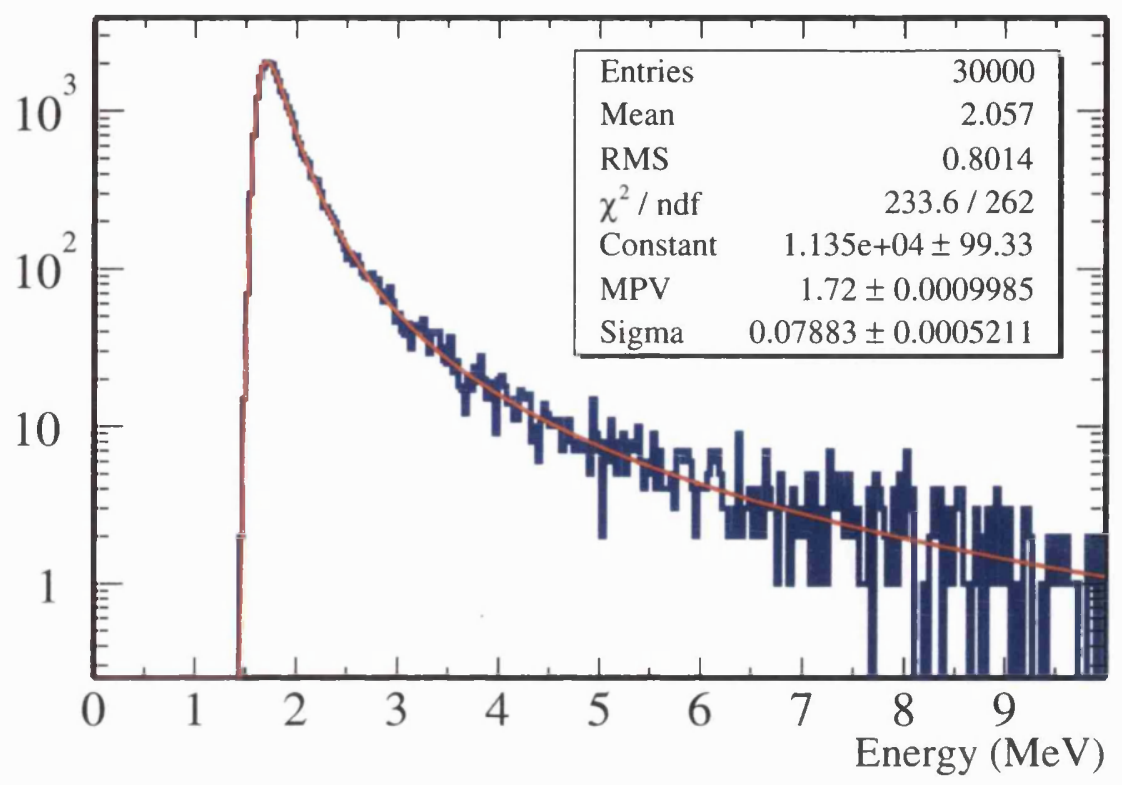

Figure A.2: GEANT simulation of the energy loss in scintillator for a $3.5 \mathrm{GeV}$ muon. A Landau distribution has been fitted to the histogram.

A function mentioned in Section 6.2.2, used to fit a constant, low light level photoelectron spectrum, employs Gaussian distributions to describe the spread after photomultiplication for each photoelectron peak. Using the phototube simulation, the actual ADC distribution for each photoelectron peak can be observed. Figure A.4 shows each of these distributions for the 1 to 10 photoelectron peaks in the proportions that they contribute in Figure A.3. It can be seen that, especially for fewer photoelectrons, the shape is Poisson rather than Gaussian, although the scale is not consistent with a Poisson distribution.

These peaks can be fitted using a scaled continuous Poisson distribution:

$$
P_{\text {scaled }}(x ; \lambda, s)=\frac{\lambda^{x / s} e^{-\lambda}}{\Gamma\left(\frac{x}{s}-1\right)}
$$




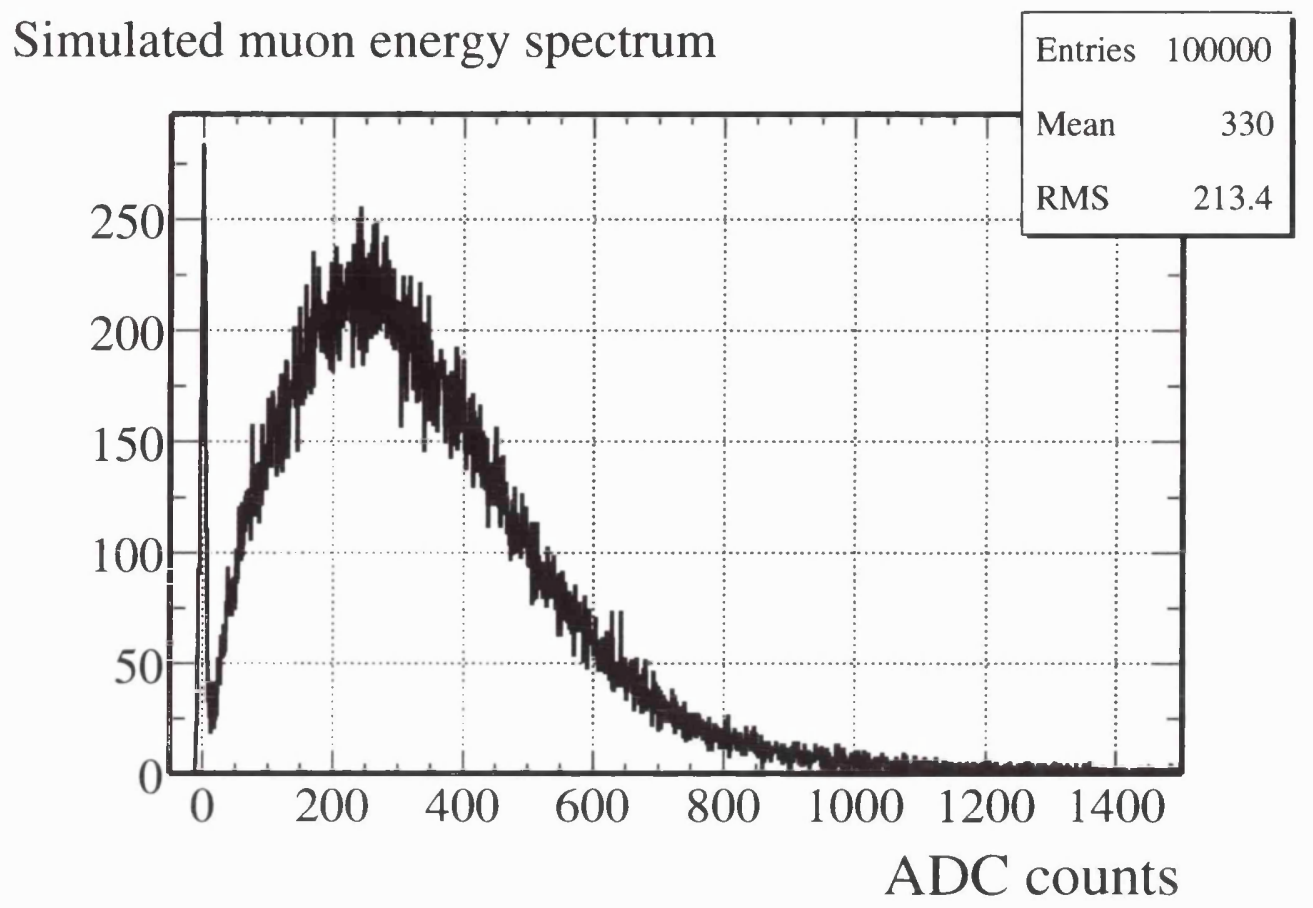

Figure A.3: A simulated muon energy spectrum showing features similar to those observed at the Calibration Detector.

where $\mathrm{x}=$ number of $\mathrm{ADC}$ counts; $\mathrm{s}=\mathrm{a}$ scale factor, which essentially reflects the fractional width of the photoelectron peak; $\lambda$ is the position of the photoelectron peak; and $\Gamma(n-1)$ is the gamma function, which is used to approximate $n$ ! for a continuous distribution. An example of the fit is shown in Figure A.5 for the first photoelectron peak.

The ideal function to describe the muon energy probability density function (PDF) in terms of ADC counts, $x$, is:

$$
\begin{aligned}
\operatorname{Prob}(x) & =L \otimes P \otimes P_{\text {scaled }} \\
& =\iint L(\lambda ; \mu, \sigma) \cdot P_{c}(n ; \lambda) \cdot P_{\text {scaled }}(x ; n, s) d \lambda d n
\end{aligned}
$$

where $L(\lambda ; \mu, \sigma)$ is the probability of obtaining $\lambda$ from a Landau distribution with a Most Probable Value, (MPV) $\mu$ and width $\sigma ; P_{c}(n ; \lambda)$ is the probability of obtaining $n$ from a continuous Poisson distribution with mean $\lambda$; and 
Simulated spectrum showing contributions from photoelectron peaks

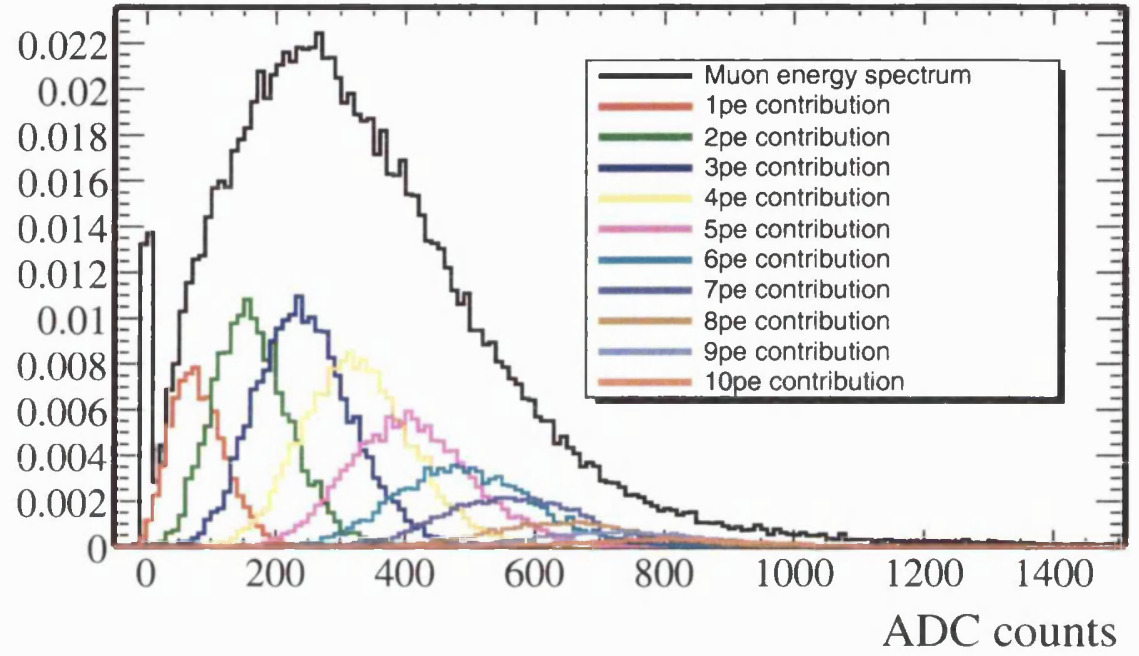

Figure A.4: A simulated muon energy spectrum showing the contributions from each of the photoelectron peaks.

$P_{\text {scaled }}(x ; \lambda, s)$ is the PDF in terms of ADC counts, $x$, for $n$ photoelectrons at the photocathode with an $\mathrm{x}$-axis scale factor $\mathrm{s}$, as discussed above.

The integrals to be solved are not straightforward because of the Landau distribution, and so an approximation to this has been adopted. The Landau distribution is replaced by the ROOT [59] adapted CERNLIB routine, and the integrals are replaced by summations. The function, $F(x)$ describes the expected distribution after photomultiplication and $x$ is in ADC counts:

$$
\begin{aligned}
F(x) & =N \cdot \operatorname{Prob}(x) \\
& \approx N \cdot \mathcal{N} \sum_{n=0}^{n_{\max }} \sum_{\lambda=\lambda_{\min }}^{\lambda_{\max }} L(\lambda ; \mu, \sigma) \cdot P(n ; \lambda) \cdot P_{\text {scaled }}(x ; n, s)
\end{aligned}
$$

$N$ is the number of entries in the spectrum; $\mathcal{N}$ is a factor required to ensure that $\operatorname{Prob}(x)$ has an area of $1 ; n$ is photoelectrons calculated $n=x / G$ where $\mathrm{G}$ is phototube gain; $P(n ; \lambda)$ is a discrete Poisson distribution returning the probability of getting $n$ for a mean $\lambda ; \lambda_{\min }$ and $\lambda_{\max }$ are the lower and upper limits between which the $L \otimes P$ integral is approximated by a 
Fit using a scaled Poisson distribution to the first photoelectron peak

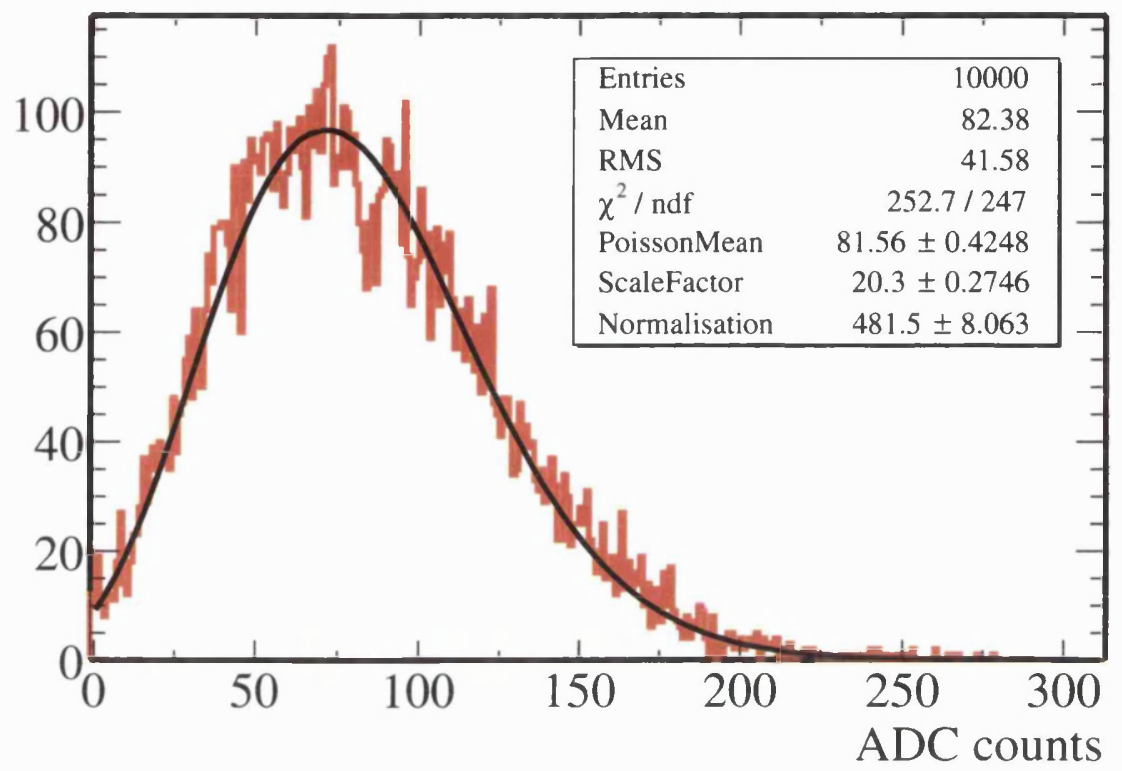

Figure A.5: A fit to the first photoelectron peak using a continuous Poisson distribution including a factor to scale the $\mathrm{x}$-axis.

summation; $n_{\max }$ is the maximum number of photoelectron peaks that need to be considered, $n_{\max }=x_{\max } / G$ where $x_{\max }$ is a limit that needs to be set according to the light level of the spectrum.

The upper limit for the summation over $n$ is set by $x_{\max }$ which is set to be the $x$-value below which $99 \%$ of the entries lie. The upper limit for the summation involving the Landau distribution is also set to be $x_{\max }$. The Landau distribution drops quickly with falling $\mathrm{x}$, so the lower limit is found by evaluating the $\mathrm{x}$-value at which the Landau probability drops below $10^{-4}$. The reason for adopting the $99 \%$ limit, rather than attempting to describe the distribution out along tail, if that the further out the function is drawn, the more loops are required for the summations. This consideration is particularly important when using the function for fitting: for the function 
to be useful, it has to be reasonably fast.

The function itself is only drawn from $0.3 G$ to $95 \%$ of the histogram range. The lower limit is adopted because for real CalDet spectra, a sparsification threshold is applied to the data. The upper limit is chosen so that the probabilities at the higher end of the function range are not significantly affected by contributions from the long tail. Since the summation only covers $99 \%$ of the spectral area, there will always be some contribution from the long tail missing in the PDF at the high end. Thus, the effect this has on the function is minimized by restricting the range over which the function is evaluated to be $95 \%$.

\section{A.4 Performing Fits with the Function}

The parameters that need to be known or found by fitting to the muon energy distribution are the gain, $\mathrm{G}$, and the scale factor, s, for the phototube; the MPV $\mu$ and the width $\sigma$ of the Landau distribution; and the number of entries in the spectrum, N. For simulated data, the phototube quantities are easily found. For real data, the gain is measured using the Light Injection system by illuminating the phototube pixels at a low, constant light level, and fitting with the function described in Section 6.2.2. This fit also returns the width of the single photoelectron peak, $\sigma_{1}$ and so the scale factor, $\mathrm{s}$, is simply obtained since, for a Poisson distribution:

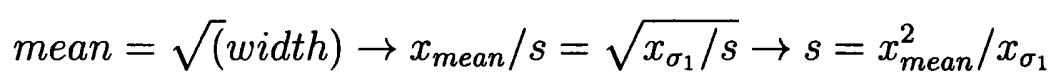

The only parameters that need to be fitted therefore, are the MPV and width of the Landau distribution. This has been done to the simulated spectrum from Figure A.3 and is shown in Figure A.6. 
Fit to simulated muon energy spectrum

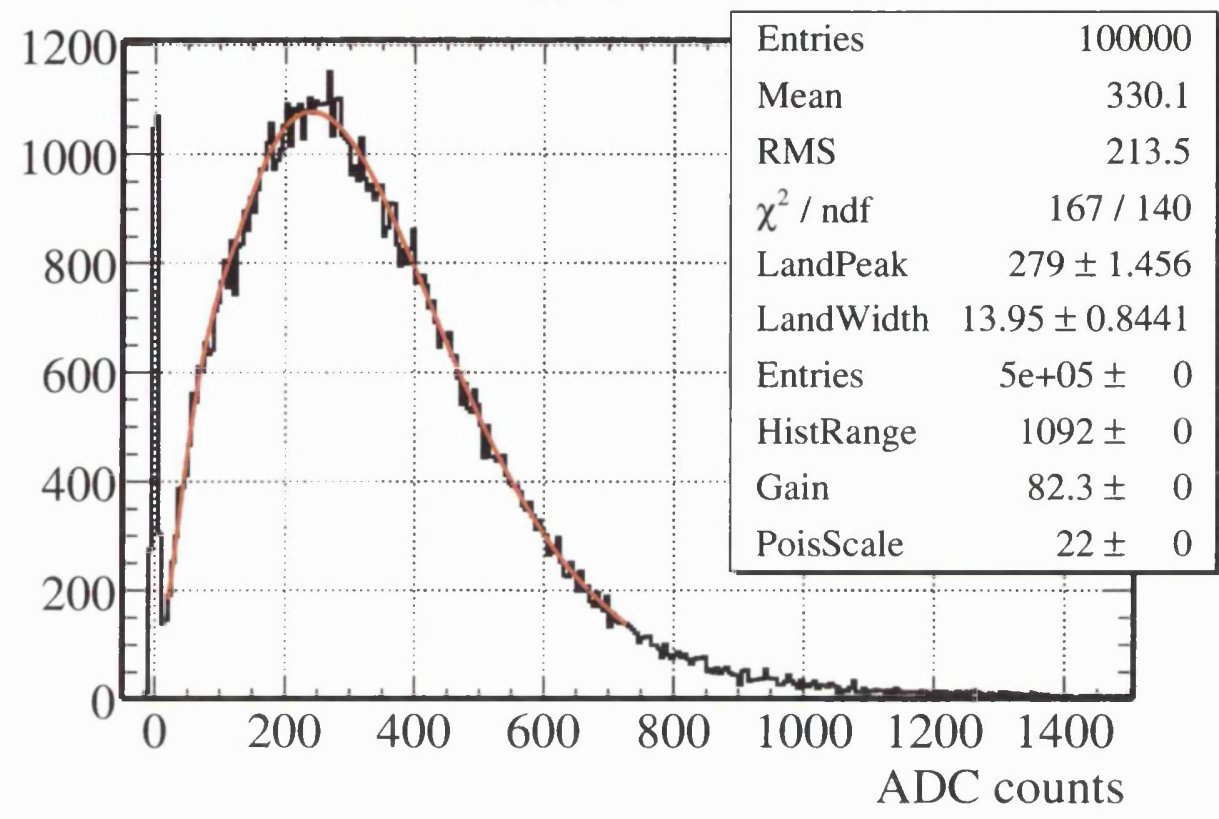

Figure A.6: A fit to a simulated muon energy spectrum.

The original parameters used to generate the spectrum were: MPV= $1.7 \mathrm{MeV}$ and width $=0.08 \mathrm{MeV}$. The number of photoelectrons per $\mathrm{MeV}$ at the phototube was estimated to be 2 based on measurements at CalDet. Therefore the expected parameters are:

$$
\begin{aligned}
M P V & =1.7 \times 2 \times G=279.5 \text { ADCs } \\
\text { width } & =0.08 \times 2 \times G=13.15 \text { ADCs }
\end{aligned}
$$

The parameters returned from the fit agree with these values within errors.

The final test is to see how the function works with a real CalDet muon energy spectrum. This is shown in Figure A.7. The fit is seen to describe the data well and the Landau parameters returned are consistent with those expected. It can be seen that the Landau width from the real data is about $50 \%$ wider than the simulated width, (recall that the simulated width comes from studies of GEANT simulated muons for the CalDet geometry). This 
difference can be ascribed to many effects which will tend to broaden the energy loss distribution, including not having a monochromatic beam and pion contamination in the muon sample, both of which will lead to a spread in $\mathrm{dE} / \mathrm{dx}$. Also, differences in collection efficiency as a function of the particle's path through the scintillator strip and inefficiencies in optical transmission will also produce similar effects.

Fit to a CalDet muon energy spectrum

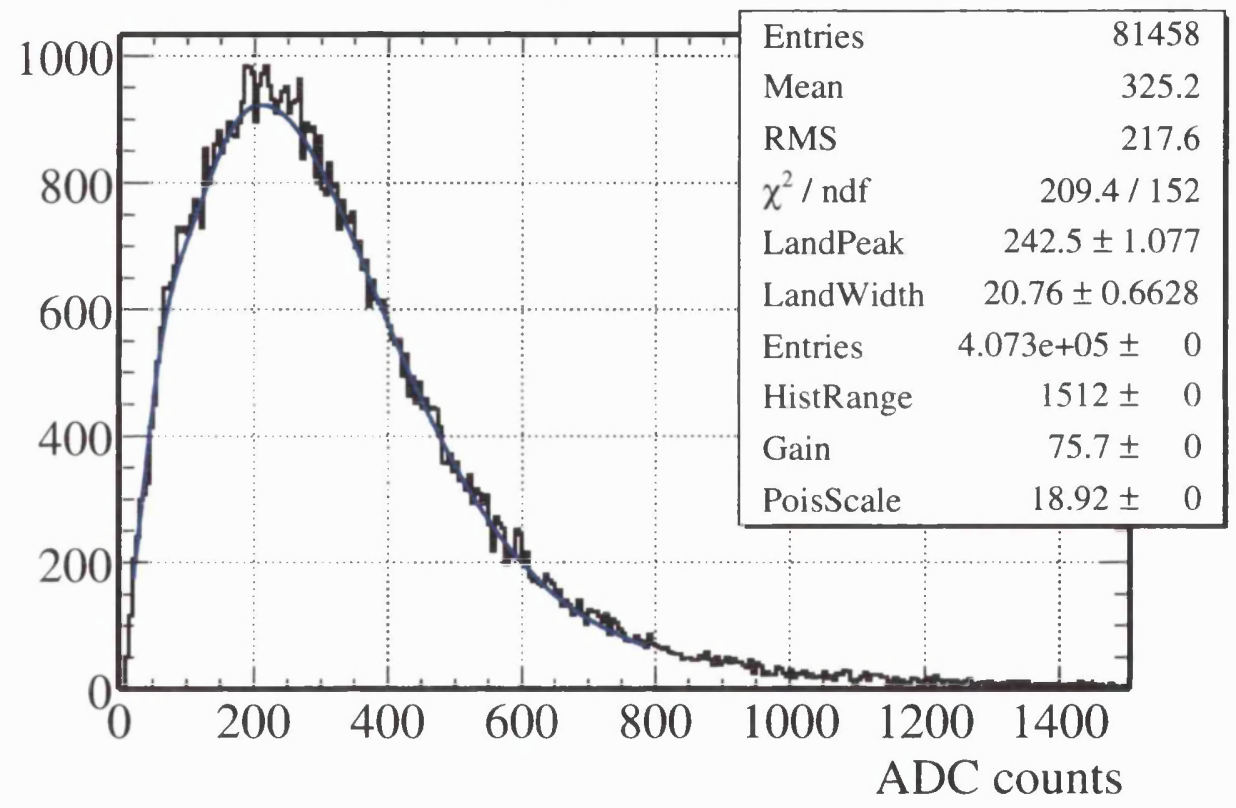

Figure A.7: A fit to a CalDet muon energy spectrum.

\section{A.5 Summary}

A fitting function has been developed with the aid of a phototube simulation and has successfully been used to describe both simulated and CalDet muon energy spectra. The use of this function is limited in two ways: with few numbers of entries the features of the energy spectra cannot be resolved 
and the fit tends to fail more frequently; the time required to perform a fit is relatively long due to the summations involved in calculating the probabilities. For these reasons, this fit is impractical at the strip-to-strip stage of the calibration. However at a later stage, for example when comparing the responses of different MINOS detectors to stopping muons, the fit may prove useful. 


\section{Bibliography}

[1] Y. Fukuda et al. [Super-Kamiokande Collaboration], Phys. Rev. Lett. 81 (1998) 1562.

[2] Y. Fukuda et al. [Super-Kamiokande Collaboration], Phys. Rev. Lett. 81 (1998) 1158.

[3] Q. R. Ahmad et al. [SNO Collaboration], Phys. Rev. Lett. 89 (2002) 011301.

[4] Q. R. Ahmad et al. [SNO Collaboration], Phys. Rev. Lett. 89 (2002) 011302.

[5] V. N. Gribov and B. Pontecorvo, Phys. Lett. B 28 (1969) 493.

[6] B. Pontecorvo, Sov. Phys. JETP 7 (1958) 172 [Zh. Eksp. Teor. Fiz. 34 (1957) 247].

[7] B. Pontecorvo, Sov. Phys. JETP 6 (1957) 429 [Zh. Eksp. Teor. Fiz. 33 (1957) 549].

[8] B. Pontecorvo, Sov. Phys. JETP 26 (1968) 984 [Zh. Eksp. Teor. Fiz. 53 (1967) 1717].

[9] K. Hagiwara et al. [Particle Data Group Collaboration], Phys. Rev. D 66 (2002) 010001. 
[10] C. S. Wu, E. Ambler, R. W. Hayward, D. D. Hoppes and R. P. Hudson, Phys. Rev. 105 (1957) 1413.

[11] M. Gell-Mann, P. Ramond and R. Slansky, Print-80-0576 (CERN).

[12] T. Yanagida, in: O. Sawada and A. Sugamoto, "Proceedings Of The Workshop On The Unified Theory And The Baryon Number In The Universe", Held At National Laboratory For High-Energy Physics (Kek), February 13-14, 1979.

[13] R. N. Mohapatra and G. Senjanovic, Phys. Rev. D 23 (1981) 165.

[14] Z. Maki, M. Nakagawa and S. Sakata, Prog. Theor. Phys. 28 (1962) 870.

[15] L. Wolfenstein, Phys. Rev. D 17 (1978) 2369.

[16] S. P. Mikheev and A. Y. Smirnov, Sov. J. Nucl. Phys. 42 (1985) 913 [Yad. Fiz. 42 (1985) 1441].

[17] C. L. Cowan, F. Reines, F. B. Harrison, H. W. Kruse and A. D. McGuire, Science 124 (1956) 103.

[18] R. J. Davis, D. S. Harmer and K. C. Hoffman, Phys. Rev. Lett. 20 (1968) 1205.

[19] J. N. Bahcall, M. H. Pinsonneault and S. Basu, Astrophys. J. 555 (2001) 990.

[20] J. N. Bahcall, http://www.sns.ias.edu/ jnb/.

[21] A. I. Abazov et al., Phys. Rev. Lett. 67 (1991) 3332.

[22] D. N. Abdurashitov et al., Phys. Rev. Lett. 77 (1996) 4708. 
[23] P. Anselmann et al. [GALLEX Collaboration], Phys. Lett. B 285 (1992) 376.

[24] W. Hampel et al. [GALLEX Collaboration], Phys. Lett. B 447 (1999) 127.

[25] K. S. Hirata et al. [Kamiokande-II Collaboration], Phys. Rev. D 44 (1991) 2241 [Erratum-ibid. D 45 (1992) 2170].

[26] Y. Fukuda et al. [Super-Kamiokande Collaboration], Phys. Rev. Lett. 82 (1999) 1810.

[27] S. Fukuda et al. [Super-Kamiokande Collaboration], Phys. Rev. Lett. 86 (2001) 5651.

[28] M. Altmann et al. [GNO Collaboration], Phys. Lett. B 490 (2000) 16.

[29] R. Becker-Szendy et al., Phys. Rev. Lett. 69 (1992) 1010.

[30] R. Becker-Szendy et al., Nucl. Phys. Proc. Suppl. 38 (1995) 331.

[31] K. S. Hirata et al. [Kamiokande-II Collaboration], Phys. Lett. B 280 (1992) 146.

[32] G. Battistoni et al., Nucl. Instrum. Meth. A 245 (1986) 277.

[33] M. Aglietta et al. [The NUSEX Collaboration], Europhys. Lett. 8 (1989) 611.

[34] K. Daum [Frejus Collaboration.], Z. Phys. C 66 (1995) 417.

[35] W. W. Allison et al. [Soudan-2 Collaboration], Phys. Lett. B 449 (1999) 137. 
[36] Y. Fukuda et al. [Super-Kamiokande Collaboration], Phys. Lett. B 433 (1998) 9 .

[37] C. Yanagisawa, Nucl. Phys. Proc. Suppl. 95 (2001) 93.

[38] M. Apollonio et al. [CHOOZ Collaboration], Phys. Lett. B 466 (1999) 415.

[39] P. Astier et al. [NOMAD Collaboration], Nucl. Phys. B 611 (2001) 3.

[40] L. Ludovici, Nucl. Phys. Proc. Suppl. 91 (2001) 177.

[41] C. Athanassopoulos et al. [LSND Collaboration], Phys. Rev. Lett. 81 (1998) 1774.

[42] H. Gemmeke et al., Nucl. Instrum. Meth. A 289 (1990) 490.

[43] LSND Collaboration, http://www.neutrino.lanl.gov/LSND/.

[44] A. O. Bazarko [BooNe Collaboration], arXiv:hep-ex/9906003.

[45] K. Nakamura [K2K Collaboration], Nucl. Phys. Proc. Suppl. 91 (2001) 203.

[46] A. Rubbia, Nucl. Phys. Proc. Suppl. 91 (2000) 223.

[47] M. Z. Akrawy et al. [OPAL Collaboration], Phys. Lett. B 231 (1989) 530.

[48] The MINOS Collaboration, "The MINOS Technical Design Report," Technical report, Fermilab 1999.

[49] The MINOS Collaboration, "The NuMI Beam Technical Design Report," Technical report, Fermilab 1999. 
[50] P. Adamson, A. Para, J. Thomas, NuMI-NOTE-SIM-0536.

[51] P. Adamson et al., arXiv:hep-ex/0204021.

[52] P. Adamson, PhD thesis, University of Sussex, 2001.

[53] V. Abramov et al., IFVE-96-91, IHEP-96-91.

[54] S. Eilerts et al., "Effects of magnetic fields on Hamamatsu R599-00-M16 PMTs," NuMI-NOTE-ELEC-0568.

[55] M. Barker et al., "Effects of Magnetic Fields on Hamamatsu R5900-00M64 PMTs," NuMI-NOTE-ELEC-0602.

[56] M. Barker et al., "A More Rigorous Description of the Influence of Magnetic Fields on Hamamatsu M64 Photomultipliers in Relation to the MINOS Experiment" NuMI-NOTE-ELEC-0650.

[57] P. Adamson et al., "Photoelectron Counting by Several Methods," NuMI-NOTE-ELEC-0661.

[58] J. Liu et al., "Basic Cross-Talk Analysis of the Data from the M16 Test Stands," NuMI-NOTE-ELEC-0735.

[59] R. Brun, F. Rademakers and S. Panacek, "ROOT, an object oriented data analysis framework," Prepared for CERN School of Computing (CSC 2000), Marathon, Greece, 17-30 Sep 2000.

[60] CERN Program Library, http://wwwinfo.cern.ch/asd/cernlib/.

[61] D. Michael, Private communication.

[62] D. E. Groom, N. V. Mokhov and S. I. Striganov, Atom. Data Nucl. Data Tabl. 78 (2001) 183. 
[63] "GEANT Detector Description and Simulation Tool," CERN Program Library, W5013 (1994).

[64] M. Bonesini, A. Marchionni, F. Pietropaolo, and T. Tabarelli de Fatis, Eur. Phys. J. C20 (2001) 13.

[65] N. V. Mokhov, Fermilab FN-628 (1995).

[66] A. J. Malensek, Fermilab FN-341 (1981).

[67] R. Hatcher, NuMI-NOTE-SIM-0479.

[68] H. Gallagher, M. Goodman, NuMI-NOTE-SIM-0112.

[69] G. J. Feldman and R. D. Cousins, Phys. Rev. D 57 (1998) 3873.

[70] D. Petyt, Private communication.

[71] R. Bernstein, Private communication.

[72] Y. Oyama [K2K Collaboration], arXiv:hep-ex/0210030. 